\title{
Cytokines in Atherosclerosis: Pathogenic and Regulatory Pathways
}

\author{
ALAIN TEDGUI AND ZIAD MALLAT
}

Institut National de la Santé et de la Recherche Médicale U. 689, Cardiovascular Research Center Lariboisiere, and University Paris 7, Paris, France

I. Introduction $\quad 516$

A. Historical perspective $\quad 516$

B. Atherosclerosis as an immunoinflammatory disease $\quad 517$

II. The Atherosclerotic Cytokine Network 518

$\begin{array}{ll}\text { A. Cytokine families } & 518\end{array}$

B. Cytokine-associated signaling pathways $\quad 519$

III. Inducers of Cytokine Production in Atherosclerosis 526

$\begin{array}{ll}\text { A. Initial trigger(s) } & 526\end{array}$

B. Secondary triggers $\quad 528$

IV. Cytokines and Cytokine Receptors in Human Atherosclerotic Plaques 535

A. Cytokine expression in plaques

B. Cellular sources of cytokines $\quad 535$

C. Biological effects of cytokines

V. Cytokine and Cytokine Receptor-Associated Modulation of Plaque Development and Stability 541

A. Proinflammatory cytokines $\quad 541$

B. Anti-inflammatory cytokines $\quad 545$

C. Chemokines/chemokine receptors $\quad 546$

$\begin{array}{ll}\text { D. Hematopoietic factors/M-CSF } & 547\end{array}$

$\begin{array}{ll}\text { E. Platelet-derived factors } & 548\end{array}$

VI. Cytokines and Adaptive Immunity in Atherosclerosis 548

A. Role of T/B cells in atherosclerosis $\quad 548$

B. Cytokines and pathogenic immune response in atherosclerosis $\quad 549$

C. Immunological tolerance and regulatory T cells 551

VII. Cytokines and Cardiovascular Risk

$\begin{array}{ll}\text { A. TNF- } \alpha & 555\end{array}$

$\begin{array}{ll}\text { B. IL-2 } & 556\end{array}$

$\begin{array}{lr}\text { C. IL-6 } & 556\end{array}$

$\begin{array}{ll}\text { D. IL-7 } & 556\end{array}$

$\begin{array}{lr}\text { E. IL-8 } & 556\end{array}$

$\begin{array}{ll}\text { F. IL-18 } & 556\end{array}$

$\begin{array}{ll}\text { G. } \mathrm{SCD} 40 \mathrm{~L} & 556 \\ \mathrm{H} & 556\end{array}$

$\begin{array}{ll}\text { H. IL-10 } & 557\end{array}$

$\begin{array}{ll}\text { I. M-CSF } & 557\end{array}$

VIII. Therapeutic Potential $\quad 557$

A. Use of anticytokines $\quad 557$

B. Targetting downstream inflammasome $\quad 557$

C. Targetting the JAK/STAT pathway 558

D. Activation of the natural anti-inflammatory intracellular pathway (SOCS) 558

E. Stimulation of Treg cells $\quad 558$

F. Stimulation of macrophage emigration from atherosclerotic lesions 559

IX. Conclusion

Tedgui, Alain, and Ziad Mallat. Cytokines in Atherosclerosis: Pathogenic and Regulatory Pathways. Physiol Rev 86: 515-581, 2006; doi:10.1152/physrev.00024.2005.-Atherosclerosis is a chronic disease of the arterial wall where both innate and adaptive immunoinflammatory mechanisms are involved. Inflammation is central at all stages of 
atherosclerosis. It is implicated in the formation of early fatty streaks, when the endothelium is activated and expresses chemokines and adhesion molecules leading to monocyte/lymphocyte recruitment and infiltration into the subendothelium. It also acts at the onset of adverse clinical vascular events, when activated cells within the plaque secrete matrix proteases that degrade extracellular matrix proteins and weaken the fibrous cap, leading to rupture and thrombus formation. Cells involved in the atherosclerotic process secrete and are activated by soluble factors, known as cytokines. Important recent advances in the comprehension of the mechanisms of atherosclerosis provided evidence that the immunoinflammatory response in atherosclerosis is modulated by regulatory pathways, in which the two anti-inflammatory cytokines interleukin-10 and transforming growth factor- $\beta$ play a critical role. The purpose of this review is to bring together the current information concerning the role of cytokines in the development, progression, and complications of atherosclerosis. Specific emphasis is placed on the contribution of pro- and anti-inflammatory cytokines to pathogenic (innate and adaptive) and regulatory immunity in the context of atherosclerosis. Based on our current knowledge of the role of cytokines in atherosclerosis, we propose some novel therapeutic strategies to combat this disease. In addition, we discuss the potential of circulating cytokine levels as biomarkers of coronary artery disease.

\section{INTRODUCTION}

Atherosclerosis is a pathological condition that underlies several important adverse vascular events including coronary artery disease (CAD), stroke, and peripheral arterial disease, responsible for most of the cardiovascular morbidity and mortality in the Western world today. Epidemiological studies indicate that the prevalence of atherosclerosis is increasing all over the world due to the adoption of Western life-style and is likely to reach epidemic proportions in the coming decades $(72,412)$.

The earliest visible lesion in the development of atherosclerosis is the fatty streak. This comprises an area of intimal thickening composed of macrophages distended by lipid droplets (known as foam cells), lymphocytes, and smooth muscle cells. The American Heart Association (AHA) Committee on Vascular Lesions provided a classification of human atherosclerotic lesions which correlate the histological lesion types, from type I to type VI, with corresponding clinical syndromes $(648,649)$. This classification should not be understood as an orderly, linear pattern of plaque progression (704). Plaques develop as a result of the accumulation of low-density lipoproteins (LDL) in the subendothelial space, followed by the diapedesis of leukocytes and formation of foam cells, proliferation of smooth muscle cells, and production of connective tissue. The landmark work of Seymour Glagov showed that the arterial wall can remodel itself in response to plaque growth by increasing its external diameter to accommodate the plaque without narrowing of the lumen (234). Thrombosis is the ultimate stage in the disease process that is responsible for clinically observable adverse events implicating coronary, cerebrovascular, and peripheral vascular beds (394). Studies indicate that in patients with atherothrombotic disease plaque formation is likely to be widespread throughout the vasculature, often affecting more than one vascular bed (93).

\section{A. Historical Perspective}

Even though atherosclerosis is reaching epidemic proportions nowadays, it is not in any way a disease specific to the modern times; it was already present in antiquity. Sir Marc Ruffer was able to identify in 1911 degenerative arterial changes suggestive of atherosclerosis in the left subclavian artery from an Egyptian mummy (583). Later on, paleopathologist A. T. Sandison, using modern technical methods for tissue fixation, confirmed that Egyptian mummies had histological evidence of atherosclerosis with lipid deposits, reduplication of the internal elastic lamina, and medial calcification in arteries (593).

Atherosclerosis is nowadays recognized as a chronic inflammatory disease of large arteries (235, 265, 395, 417, 578). Remarkably, the very first description of the cause of angina pectoris referred to inflammation. Yet, the belief in this notion was subjected to peaks and troughs from early dates up to recent times.

According to the historian J. O. Leibowitz (381), the Italian surgeon and anatomist Antonio Scarpa (1752-1832) was the first to present an anatomopathological description of arterial wall degeneration in full detail. In his 1804 monograph on aneurysms, Scarpa opposed the view that a dilatation of the aorta was the intrinsic cause of an aneurysm leading to rupture. He emphasizes that “... especially the internal coat is subject, from slow internal cause, to an ulcerated and steatomatous disorganization, as well as to a squamous and earthy rigidity and brittleness," introducing the concept of an underlying metabolic disorder in the process of atherosclerosis, rather than the theory of inflammation that already prevailed at that time, the expression "heart abscess" being frequently used to describe heart pathology (reviewed in Ref. 381).

The term atheroma, derived from Greek and meaning "porridge," was first proposed by Albrecht von Haller in 1755 to designate the degenerative process observed in the intima of arteries. London surgeon Joseph Hodgson 
(1788-1869) published in 1815 his Treatise on the Diseases of Arteries and Veins in which he claimed that inflammation was the underlying cause of atheromatous arteries. But thereafter, most of pathologists of the 19th century following Carl Rokitanski (1804-1878) abandoned the view that inflammation was an etiological factor and considered that atherosclerosis was a degenerative process, with intimal proliferation of connective tissue and calcification, best described by the term arteriosclerosis proposed in 1833 by French pathologist Jean Lobstein (1777-1835). However, German pathologist Rudolf Virchow (1821-1902), a leading authority of his day in pathology and the greatest contributor to the notion of thrombosis, considered atheroma as a chronic inflammatory disease of the intima, that he called "chronic endarteritis deformans". In his opinion, the accumulation of lipids was a late manifestation of atheroma (701). Finally, the Leipzig pathologist Marchand in 1904 first used the term atherosclerosis, which since has been widely adopted, instead of arteriosclerosis, to designate the degenerative process of the intimal layer of the arteries.

Until the beginning of the 20th century, the theories put forward to explain the pathogenesis of atherosclerosis remained purely descriptive and were based on the anatomical observation of human atherosclerotic vessels. A first revolution in the mechanistic assessment of atherosclerosis was initiated in 1908 when the Russian scientist Alexander Ignatowski showed that experimental atherosclerosis could be induced in rabbits by feeding them a diet of milk and egg yolk (301). Soon thereafter, in 1913, N. Anitschkov and S. Chalatov reproduced experimental atherosclerosis by adding pure cholesterol to rabbit food (21). This gave rise to the lipid theory of atherosclerosis that predominated for most of the 20th century. The next significant leap only came during the 1970s when Brown and Goldstein showed that the LDL receptor that they had discovered, a cell surface protein that binds LDL and removes them from blood (reviewed in Ref. 88), is not involved in macrophage foam-cell formation and proposed that a macrophage receptor that recognized acetylated LDL plays a key role in this process (237). Subsequently, during the $1980 \mathrm{~s}$, the central role of oxidized LDL (oxLDL) in the pathogenesis of atherosclerosis was exposed by Daniel Steinberg and his group (650), and a number of scavenger receptors mediating their uptake by macrophages were identified (reviewed in Ref. 387). The model of the Watanabe heritable hyperlipidemic (WHHL) rabbit, introduced in 1980 (726) was particularly useful in establishing the role of oxLDL in atherogenesis. A second revolution occurred at the beginning of the 1990s when mouse models of atherosclerosis, apolipoprotein $\mathrm{E}$ (apoE)- and LDL receptor (LDLr)-deficient mice, were derived by homologous recombination techniques (304, $306,543,784)$. In contrast to the previous models, mice lacking functional apoE or LDLr genes were shown to develop widely distributed arterial lesions that progress from foam cell-rich fatty streaks to fibro-proliferative plaques with lipid/necrotic cores, typical of the spectrum of human lesions $(305,487,564)$. The possibility of abolishing the expression of a single gene of interest, or of overexpressing it, in these mouse models opened a new era of atherosclerosis research at a mechanistic level.

\section{B. Atherosclerosis as an Immunoinflammatory Disease}

A ripple in the lipid theory appeared in the mid 1970s, when Russel Ross developed his popular "response to injury" hypothesis of atherogenesis, postulating that atherosclerotic lesions arise as a result of focal injury to the arterial endothelium, followed by adherence and aggregation of platelets (580). During the resulting release reaction, platelet-derived growth factor (PDGF) is secreted from the platelets and promotes the proliferative response of smooth muscle cells (SMC). Uncontrolled exuberant SMC proliferation was believed to eventually cause artery occlusion. SMC were considered at that time to be the main promoter of atherosclerotic lesion formation. Instead, it has since been clearly established that SMC proliferation in the plaque is rather modest, and actually tends to be beneficial since it contributes to plaque stabilization $(158,731)$. In addition, the endothelium actually remains morphologically intact during the development of atherosclerosis $(197,578)$, although it is activated and directly involved in the immunoinflammatory response. Poole and Florey (547) were the first to observe that soon after initiation of cholesterol feeding in rabbits, monocytes adhere to the endothelium and migrate through the yet intact endothelial monolayer. Michael Gimbrone first proposed the concept of endothelial dysfunction that acknowledged the central role of the normal endothelium in protecting against atherosclerosis while hypothesizing that its cellular functions were altered, "activated" in the disease (232). Ross revisited his "response to injury" theory in 1986 (579) considering that "subtle endothelial injury" was the primum movens in atherosclerosis, and published in 1999 in the New England Journal of Medicine a remarkable review entitled: "Atherosclerosis: a chronic inflammatory disease" (578). The view that atherosclerosis is indeed a chronic inflammatory disease initiated by monocyte/lymphocyte adhesion to activated endothelial cells (EC) is now widely accepted and substantiated by experimental and clinical observations. Several excellent reviews have been published on the theme of atherosclerosis and inflammation since the founding Ross review (52, 235, 265, 395, $417,578)$.

Instrumental in the change of opinion regarding the role of inflammation and immunity, rather than SMC pro- 
liferation, in the pathogenesis of atherosclerosis was the precise identification of the cell components of human atherosclerotic plaques using modern immunohistochemical techniques by Göran Hansson and colleagues (316). Histologically, the lipid-laden foam cells of the fatty streak, which characterizes the plaque at an early stage, are derived from macrophages. In time, the lipid/necrotic core is covered with fibrous tissue composed mainly of $\alpha$-actin positive SMC, and thus forms the fibrolipid plaque. Rather large amounts of T lymphocytes, 20\%, are found as well, surrounding the plaque and in the fibrous cap, pointing to a role of immunity in atherosclerosis $(268,316)$.

Also determinant in the understanding of the pathogenesis of atherosclerosis were the works by the pathologists Michael Davies (158, 159) and Erling Falk (198), later confirmed and extended by the group of Renu Virmani (704), in their quest for the causes of acute coronary syndromes. Their works emphasized that coronary atherosclerotic plaques exist under two major phenotypes: 1) stable plaques, characterized for the most part by a thick fibrous cap isolating a relatively small lipid core from the lumen, which are associated with a very low risk of thromboembolic complications; and 2) unstable (or vulnerable) plaques, most of which are characterized by a large lipid core covered by a thin fibrous cap prone to rupture and thrombus formation, and which are thought to be associated with a higher risk for thromboembolic complications (218). Analysis of culprit atherosclerotic lesions in patients with acute myocardial infarction revealed that inflammation is crucially determinant in precipitating plaque rupture and some forms of superficial plaque erosion (157, 353, 690).

Virmani uncovered another mechanism of coronary thrombosis occurring in unruptured noninflammatory plaques, described as plaque erosion $(199,703)$. Eroded plaques differ from ruptured plaques in that they have a base rich in proteoglycans and SMCs. These lesions are more often seen in younger individuals and women, they are associated with less luminal narrowing and less calcification, and they are less likely to have foci of macrophages and T cells compared with ruptured plaque (199). We recently provided experimental evidence that endothelial apoptosis might be a major determinant of plaque erosion $(182,679)$.

Inflammation, which "is a complex set of interactions among soluble factors and cells that can arise in any tissue in response to traumatic, infectious, postischemic, toxic or autoimmune injury" (493) appears to be involved at all stages of atherosclerosis. It is implicated in the formation of early fatty streaks, when the endothelium is activated and expresses chemokines, including monocyte chemotactic protein (MCP)-1 and interleukin (IL)-8, and adhesion molecules, including intercellular adhesion molecule (ICAM)-1, vascular adhesion molecule (VCAM)-1, E- and P-selectin, leading to monocyte/lymphocyte recruitment and infiltration into the subendothelium (265). It also acts at the onset of adverse clinical vascular events, when activated cells within the plaque secrete matrix proteases that degrade extracellular matrix proteins and fragilize the fibrous cap, leading to rupture and thrombus formation (399). Cells involved in the atherosclerotic process include vascular (endothelial and smooth muscle) cells, monocytes/macrophages, lymphocytes (T, B, NKT), dendritic cells, and mast cells. They secrete or are stimulated by soluble factors including peptides, glycoproteins, proteases, and a set of cytokines.

The purpose of this review is to bring together the current information concerning the role of cytokines in the development, progression, and complications of atherosclerosis. Specific emphasis is placed on the contribution of pro- and anti-inflammatory cytokines, in modulating innate, adaptive, and regulatory immunity in the context of atherosclerosis. In addition, we discuss the potential of the circulating cytokine levels as biomarkers of (coronary) artery disease. Finally, we propose some novel therapeutic strategies targeting the cytokine network to combat atherosclerosis.

\section{THE ATHEROSCLEROTIC CYTOKINE NETWORK}

\section{A. Cytokine Families}

Stanley Cohen introduced for the first time the word cytokine in $1974(132,133)$. Until then the term lymphokine, proposed by Dudley Dumonde in 1969, had been used to designate lymphocyte-derived factors and more generally proteins secreted from a variety of cell sources, affecting the growth or function of many types of cells, collectively (181). At the second International Lymphokine Workshop held in 1979, the name interleukin was proposed to characterize proteins with "the ability to act as communication signals between different populations of leukocytes" (473). Later on in 1989, Balkwill and Burke (33) defined cytokine as "one term for a group of protein cell regulators, variously called lymphokines, monokines, interleukins, interferons (we should add "chemokines"), which are produced by a wide variety of cells in the body, play an important role in many physiological responses, are involved in the pathophysiology of a range of diseases, and have therapeutic potential."

Nowadays, the cytokines consist of more than 50 secreted factors involved in intercellular communication, which regulate fundamental biological processes including body growth, lactation, adiposity, and hematopoiesis (77). Cytokines are clustered into several classes: interleukins (33 have been identified to date), tumor necrosis factors (TNF), interferons (IFN), colony stimulating fac- 
tors (CSF), transforming growth factors (TGF), and chemokines. They are especially important for regulating inflammatory and immune responses and have crucial functions in controlling both innate and adaptive immunity. The predominant actors in adaptive immunity, helper$\mathrm{T}(\mathrm{Th})$ cells, have been categorized on the basis of the pattern of cytokines that they can secrete, resulting in either a cell-mediated immune response (Th1) associated with IL-2 and IFN- $\gamma$ secretion, or a humoral immune response (Th2), associated with IL-4, IL-5, IL-10, and IL-13 secretion.

Cytokines are categorized according to the structural homology of their receptors as class I or class II cytokines (77, 369) (Table 1). Most ILs, CSFs, and IFNs belong to one of these two classes of cytokines, which mediate their effects through the Janus kinase-signal transducers and activators of transcription (JAK-STAT) pathway. Three other major cytokine families encompass the IL-1 family (including IL-1 $\alpha$, IL-1 $\beta$, IL-1ra, and IL-18), TNF family, and TGF- $\beta$ superfamily (Table 1 ). IL- 1 and TNF family members activate the nuclear factor- $\kappa \mathrm{B}(\mathrm{NF}-\kappa \mathrm{B})$ and mitogenactivated protein (MAP) kinase signaling pathways, while TGF- $\beta$ superfamily members activate signaling proteins of the Smad family.

The TGF- $\beta$ superfamily is composed of many multifunctional cytokines including TGF- $\beta 1-2-3$, activins, inhibins, anti-Müllerian hormone (AMH), bone morphogenetic proteins (BMPs), and myostatin (540). TGF- $\beta$ family members are secreted as inactive complexes bound to the latency-associated peptide (LAP), a protein derived from the $\mathrm{NH}_{2}$-terminal region of the TGF- $\beta$ gene product. The LAP forms covalent bonds with the latent TGF- $\beta$ binding proteins (LTBPs), high-molecular-weight proteins of which four different isoforms exist (571). The resulting large latent complexes are sequestered within the extracellular matrix. Proteases in the extracellular matrix can digest LTBP, dissociating LAP from TGF- $\beta$.

Cytokines share a number of specific features.

1) They show pleiotropic activities: a cytokine can trigger several different cellular responses depending on cell type, timing, and context.

2) They act synergistically: the association of two cytokines (for example, IL-12 and IL-18, TNF- $\alpha$ and IFN- $\gamma$, MCP-1 and IL-8) markedly amplifies their activity. This also holds true when a cytokine induces the expression of (an)other cytokine receptor(s).

3) They act in an autocrine, paracrine, or juxtacrine manner: cytokines can stimulate on the cells that produce them, or adjacent cells, or they can intervene through direct cell-cell interaction. This local mode of action sets cytokines apart from classical hormones.

4) They commonly share cytokine receptor subunits: for example, several members of the IL-2 family (IL-7, IL-9, IL-15, IL-21) share the IL-2 receptor $\gamma$-chain, the IL-6 family cytokines share the gp130 subunit, and the three
IFN- $\lambda$ isoforms utilize a heterodimeric receptor composed of its specific receptor subunit IFN- $\lambda \mathrm{R}$ (or IL-28R $\alpha$ ) and the subunit IL-10R2 of the IL-10R, also shared with IL-10 and the IL-10-related cytokines, IL-22 and IL-26.

One must admit that many of these properties are also shared by growth factors. However, one difference is that the production of growth factors, including PDGF, epidermal growth factor (EGF), fibroblast growth factor (FGF), and vascular endothelial growth factor (VEGF), tends to be constitutive and is not as tightly regulated as that of cytokines. Also, the target cells of growth factors are mainly nonimmune.

Cytokines are often classified according to their pro(TNF, IL-1, IL-12, IL-18, IFN- $\gamma$ ) or anti-inflammatory (IL-4, IL-10, IL-13, TGF- $\beta$ ) activities. In light of the data obtained from experimental and clinical studies, described below, regarding the pathophysiological role of cytokines in atherosclerosis, we propose to cluster cytokines as pro- or antiatherogenic (Table 2).

\section{B. Cytokine-Associated Signaling Pathways}

\section{1. $N F-\kappa B$}

The NF- $\kappa$ B pathway is one of the main signaling pathways activated in response to proinflammatory cytokines, including TNF- $\alpha$, IL-1, and IL-18, as well as following activation of the Toll-like receptors (TLR) by the pattern recognition of pathogen-associated molecular patterns (PAMPs). Activation of this pathway plays a central role in inflammation through the regulation of genes encoding pro-inflammatory cytokines, adhesion molecules, chemokines, growth factors, and inducible enzymes such as cyclooxygenase-2 (COX2) and inducible nitric oxide synthase (iNOS) (166). NF- $\kappa \mathrm{B}$ is a dimeric transcription factor formed by the hetero- or homodimerization of proteins of the Rel family, including $\mathrm{p} 50$ and p65. In its inactive form NF- $\kappa \mathrm{B}$ is bound to inhibitor of $\kappa \mathrm{B}(\mathrm{I}-\kappa \mathrm{B} \alpha / \beta)$ in the cytoplasm. Proinflammatory cytokines and pathogens act through distinct signaling pathways that converge on the activation of an $\mathrm{I} \kappa \mathrm{B}$ kinase (IKK) complex containing two kinases IKK1/IKK $\alpha$ and IKK2/IKK $\beta$, and the regulatory protein NEMO (NF- $\kappa \mathrm{B}$ essential modifier, also named IKK $\gamma$ ) (762); IKK activation initiates $\mathrm{I} \kappa \mathrm{B} \alpha / \beta$ phosphorylation at specific $\mathrm{NH}_{2}$-terminal serine residues (782). Phosphorylated $\mathrm{I} \kappa \mathrm{B}$ is then ubiquitinated, leading to its degradation by the $26 \mathrm{~S}$ proteasome. This releases $\mathrm{NF}-\kappa \mathrm{B}$ dimers from the cytoplasmic NF- $\kappa \mathrm{B}-\mathrm{I} \kappa \mathrm{B}$ complex, allowing them to translocate to the nucleus (Fig. 1). Once in the nucleus, NF- $\kappa \mathrm{B}$ binds to $\kappa \mathrm{B}$ enhancer elements on specific genes promoting transcription. Targets genes of $\mathrm{NF}-\kappa \mathrm{B}$ include $\mathrm{I} \kappa \mathrm{B} \alpha$, the synthesis of which ensures that $\mathrm{NF}-\kappa \mathrm{B}$ is transiently activated. This negative-feedback regulation gives rise to oscillations in $\mathrm{NF}-\kappa \mathrm{B}$ translocation (496). 
TABLE 1. Cytokine classification

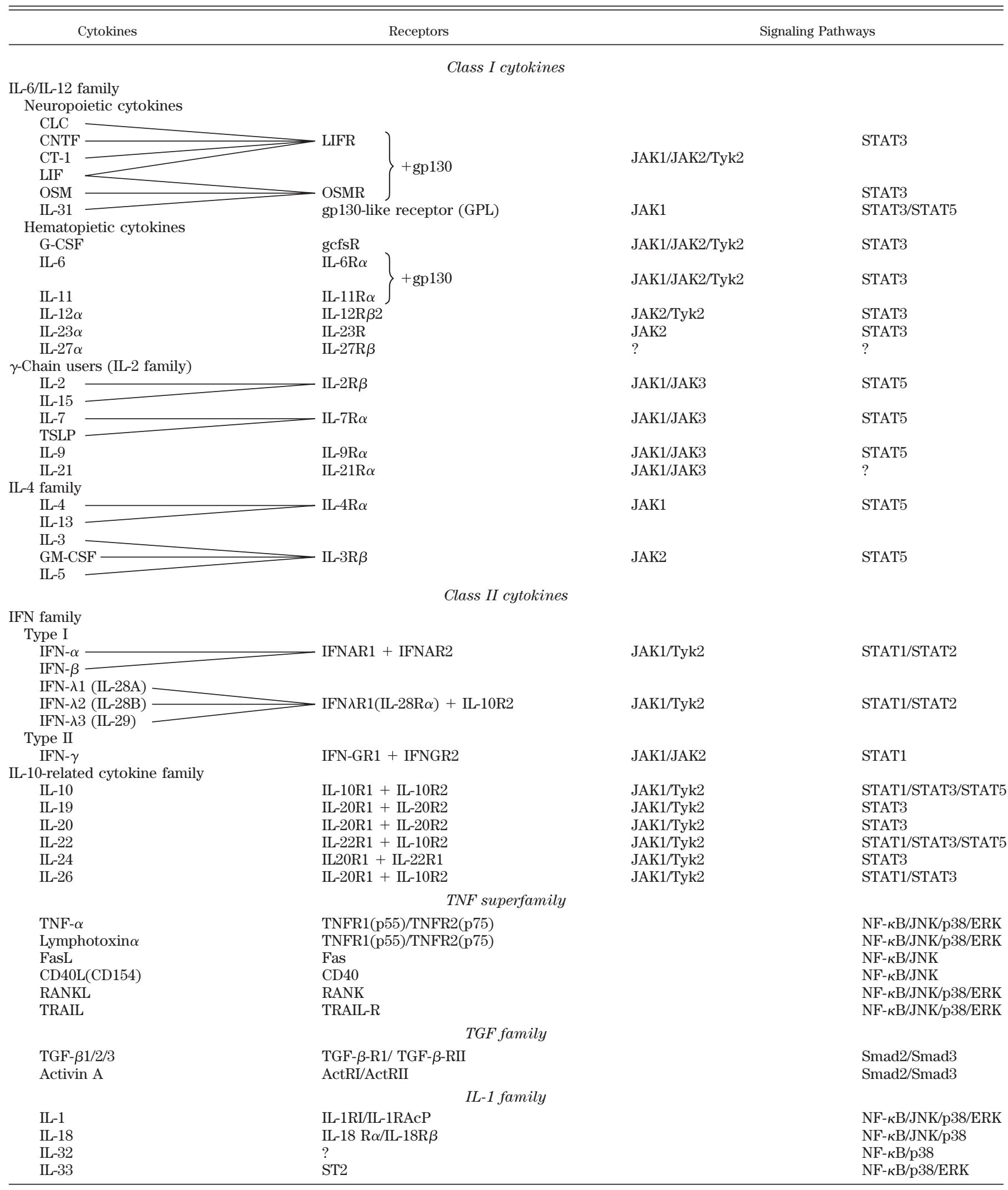

CLC, CT-1-like factor; LIF, leukemia inhibitory factor; CNTF, ciliary neurotrophic factor; CT-1, cardiotrophin-1; OSM, oncostatin M; G-CSF, granulocyte-colony stimulating factor; TSLP, thymic stromal lymphopoietin; RANK, receptor activator of NF- $\mathrm{k}$; RANKL, RANK ligand; ActRI, activin type I receptor; IL-1RAcP, IL-1 receptor accessory protein. 
TABLE 2. Pro- and antiatherogenic cytokines

\begin{tabular}{ll}
\hline \hline \multicolumn{1}{c}{ Proatherogenic Cytokines } & Antiatherogenic Cytokines \\
\hline TNFR family & \\
TNF- $\alpha$ & \\
Lymphotoxin & \\
Osteoprotegerin & \\
CD40L & \\
IL-1 family & IL-1ra \\
IL-1 & IL-18BP \\
IL-18 & \\
Class I cytokines & \\
IL-2 & IL-6 \\
IL-4 & IL-9 \\
IL-6 & \\
IL-12 & IL-10 \\
Class II cytokines & \\
IFN- $\gamma$ & \\
Hematopoietic factors & \\
M-CSF & \\
Chemokines/chemokine receptors & \\
IL-8/CXCR2 & \\
MCP-1/CCR2 & \\
Fractalkine/CX3CR1 & TGF- $\beta$ \\
RANTES & \\
MIF & \\
Bone-associated cytokines & \\
Osteopontin & \\
TGF- $\beta$ family & \\
\hline
\end{tabular}

TNF, tumor necrosis factor; IFN, interferon; TGF, transforming growth factor.

$\mathrm{NF}-\kappa \mathrm{B}$ is a redox-sensitive transcription factor, and the intracellular redox status of the cell is extremely important in the regulation of NF- $\kappa \mathrm{B}$ activity (reviewed in Ref. 311). Antioxidants, such as aspirin, $N$-acetylcysteine (NAC), and flavonoids can therefore inhibit the activation of NF- $\kappa$ B. A number of natural constitutive or inducible pathways inhibiting NF- $\kappa \mathrm{B}$ activity also exist (see review in Ref. 669). For example, A1 and A20, two cytoprotective genes, are induced in response to inflammatory stimuli to protect EC from exaggerated activation and from undergoing apoptosis even when NF- $\kappa \mathrm{B}$ is blocked (139). A20 terminates $\mathrm{NF}-\kappa \mathrm{B}$ activation by promoting reaccumulation of $\mathrm{I} \kappa \mathrm{B}$ through its interaction with proteins involved in $\mathrm{TNF}-\alpha$ signaling upstream of $\mathrm{I} \kappa \mathrm{B}$ degradation (375). Consequently, A20-deficient mice fail to terminate TNFinduced NF- $\kappa \mathrm{B}$ activity, having a persistently active IKK complex that prevents reaccumulation of $\mathrm{I} \kappa \mathrm{B}$ protein, are hypersensitive to TNF- $\alpha$, and suffer from severe inflammation. The inducible form of the heme oxygenase (HO-1) is another example of endogenous anti-inflammatory pathway induced in response to inflammatory stimuli. HO- 1 can be upregulated in human EC by TNF and IL-1 (674), and HO-1 possesses potent antiapoptotic and antiinflammatory properties (742). HO-1 deficiency in humans is associated with the presence of severe and persistent endothelial damage (761). The anti-inflammatory properties of HO-1 seem to be related to an inhibitory action on P- and E-selectin expression on EC (688).
Activated NF- $\kappa \mathrm{B}$ has been identified in SMC, macrophages, and EC of human atherosclerotic lesions $(78,82$, $480)$. Enhanced endothelial activation of NF- $\kappa \mathrm{B}$ has been shown to occur in LDLr-deficient mice very early on following a high-fat diet, in regions of the proximal aorta with high probability for atherosclerotic lesion development (262). Furthermore, supershift analysis in cells isolated from human carotid atherosclerotic plaques, composed in majority of macrophages and SMC, demonstrate that activated NF- $\kappa \mathrm{B}$ consists of $\mathrm{p} 65$, c-Rel, and $\mathrm{p} 50$, but not relB or p52 subunits (480). NF- $\kappa$ B activation in these cells controls the expression of proinflammatory cytokines TNF- $\alpha$; IL-6 and IL-8; matrix metalloproteinases (MMP)-1, -3, and -9; and tissue factor (TF), as shown by their selective inhibition following blockade of the NF- $\kappa \mathrm{B}$ pathway by overexpression of $\mathrm{I} \kappa \mathrm{B} \alpha$ or dominant-negative IKK-2 (480). Interestingly, in this study NF- $\kappa$ B inactivation did not affect the expression of the anti-inflammatory cytokine IL-10 or the matrix metalloproteinase inhibitor TIMP-1.

The actual in vivo role of the NF- $\kappa \mathrm{B}$ pathway has recently been addressed in experimental models of atherosclerosis. Kanters et al. (324), using LDLr-deficient mice with a cell-specific deletion of IKK2 preventing $\mathrm{NF}-\kappa \mathrm{B}$ activation in macrophages, unexpectedly found increased atherosclerotic lesion formation and inflammation in these animals. This result was associated with a significant reduction in the anti-inflammatory and antiatherogenic cytokine IL-10, suggesting that a certain level of NF- $\kappa \mathrm{B}$ activation is required to modulate the inflammatory reaction and counteract proatherogenic responses (Fig. 2). This finding is in favor of a central role for $\mathrm{NF}-\kappa \mathrm{B}$ in the induction of "protective" antiapoptotic and anti-inflammatory genes, critical to the resolution of the inflammatory process (374). However, the detrimental effect of $\mathrm{NF}-\kappa \mathrm{B}$ inhibition in atherogenesis is likely to depend on how NF- $\kappa \mathrm{B}$ activity is inhibited. In a subsequent study, Kanters et al. (323) examined the effects of hematopoietic NF- $\kappa \mathrm{B} 1$ (the p50 subunit of NF- $\kappa \mathrm{B}$ ) deficiency in the development of atherosclerotic lesions, transplanting bone marrow from mice deficient in NF- $\kappa \mathrm{B} 1$ into irradiated $\mathrm{LDLr}^{-/-}$mice. Instead of promoting the formation of larger inflammatory lesions, as was the case with specific IKK2 deficiency in macrophages, hematopoietic NF- $\kappa \mathrm{B} 1$ deficiency was associated with a significant decrease in lesion size, despite enhanced accumulation of $\mathrm{T}$ and $\mathrm{B}$ lymphocytes within the lesions. This could be explained, at least in part, by the observation that in contrast to IKK2 deficiency, NF- $\kappa \mathrm{B} 1$ deficiency did not alter the inflammatory balance in favor of a proatherogenic phenotype. Despite increased TNF- $\alpha$ expression by NF- $\kappa$ B1-deficient macrophages, other major proatherogenic molecules such as MCP-1 were downregulated, whereas critical antiatherogenic factors such as IL-10 were significantly upregulated. 


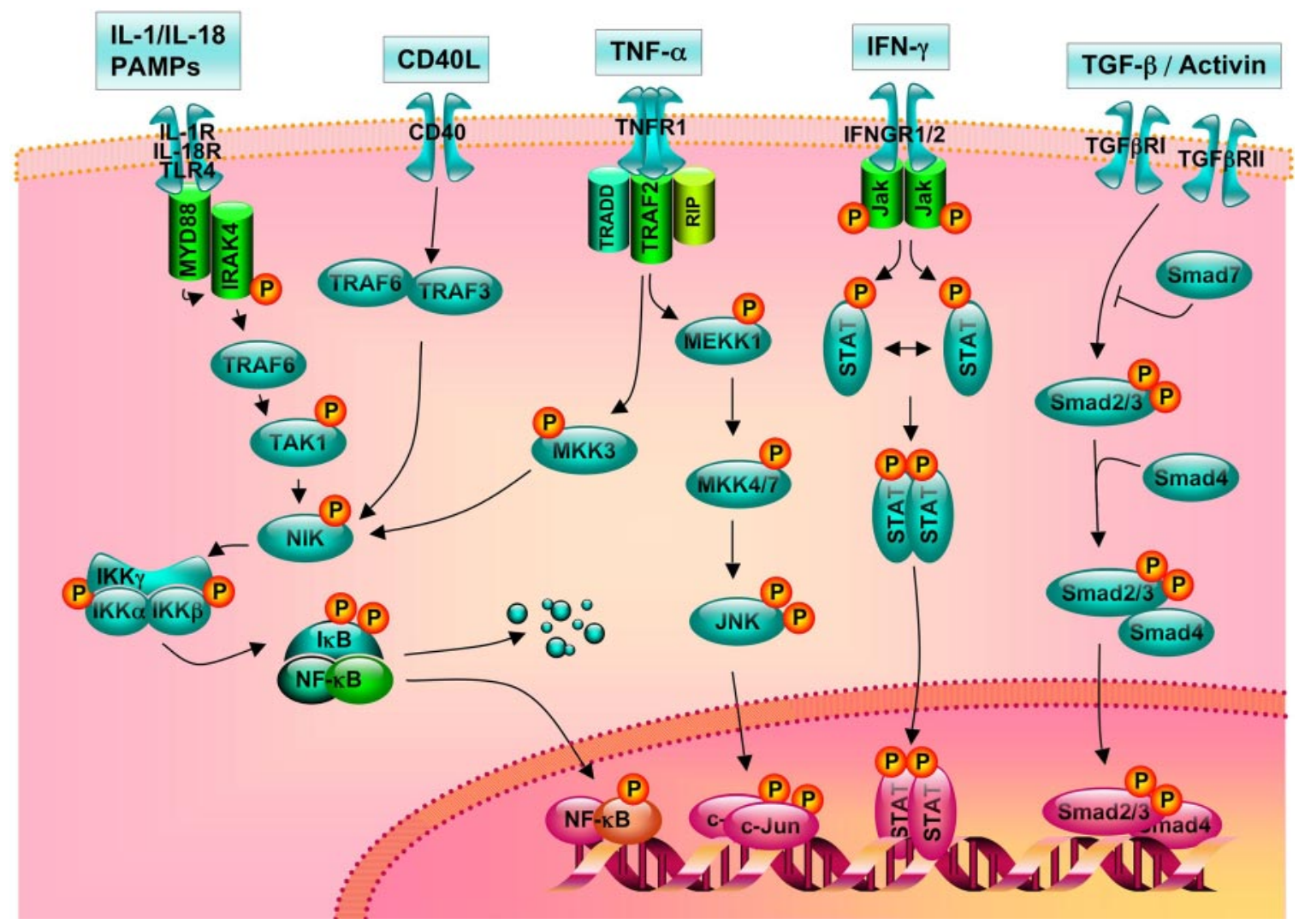

FIG. 1. Principal signaling pathways involved in atherogenesis. Proinflammatory cytokines (IL-1, IL-18) and pathogens (represented as pathogen-associated molecular patterns, PAMP), as well as nonpathogen activators of TLR, act through distinct signaling pathways that converge on the activation of NF- $\kappa$ B. MyD88 functions as an adaptor between receptors of the TLR or IL-1R families and downstream signaling kinases. Following association of MyD88 with IL-1-associated kinase IRAK-4, IRAK-4 is autophosphorylated, dissociates from the receptor complex, and interacts with TNF-receptor-associated factor-6 (TRAF-6), which also mediates CD40 signaling. Once activated, TRAF6 associates with the MAP3 kinase TAK1 (716). From TAK1, two signaling pathways diverge; one ultimately leads to NF- $\kappa \mathrm{B}$ activation and the other to MAP kinase activation. In its inactive form, NF- $\kappa \mathrm{B}$ is bound to inhibitor of $\kappa \mathrm{B}(\mathrm{IK}-\kappa \mathrm{B} \alpha / \beta)$ in the cytoplasm and consists of an I $\kappa \mathrm{B}$ kinase (IKK) complex containing two kinases IKK $\alpha$ and IKK $\beta$, and the regulatory protein IKK $\gamma$ (also named NEMO); IKK activation initiates I $\kappa$ B $\alpha / \beta$ phosphorylation. Phosphorylated I $\kappa \mathrm{B}$ is then ubiquitinated, leading to its degradation by the $26 \mathrm{~S}$ proteasome. This releases NF- $\kappa \mathrm{B}$ dimers from the cytoplasmic NF- $\kappa \mathrm{B}-\mathrm{I} \kappa \mathrm{B}$ complex, allowing them to translocate to the nucleus. JNK phosphorylation is mediated by two MAPK kinases (MAPKKs), MKK4 and MKK7, that they can cooperatively activate JNK. Both kinases are required for full activation of JNK by environmental stressors, and MKK7 is essential for JNK activation by TNR engagement. Tyrosine phosphorylation activates the cytosolic inactive STATs, resulting in their nuclear translocation and gene activation. This pathway was originally found to be activated by IFNs, but a number of cytokines, growth factors, and hormonal factors also activate JAK and/or STAT proteins. IFN- $\gamma$ utilizes JAK1 and JAK2, and usually activates STAT1. TGF- $\beta$-triggered signals are transduced by proteins belonging to the Smad (for vertebrate homologs of Sma and $\mathrm{Mad}$ ) family. The type I receptor recognizes and phosphorylates Smad2 and Smad3, which associates with Smad4, forming complexes that participate in DNA binding and recruitment of transcription factors. Smad3 may also have antagonistic properties, as it plays a critical role in TGF- $\beta$-dependent repression of vascular inflammation by inhibiting AP-1 activity. Smad7 inhibits Smad2 and Smad3 phosphorylation.

Decreased MCP-1 production and increased IL-10 expression may have contributed to the limitation of plaque size despite enhanced accumulation of $\mathrm{T}$ cells. Another plausible explanation for reduced lesion development in NF- $\kappa$ B1-deficient animals could be a defect in the uptake of oxLDL by macrophages, as characteristic foam cells were absent in NF- $\kappa \mathrm{B} 1$-deficient lesions. Moreover, both scavenger receptor class A (SR-A) expression and uptake of oxLDL were significantly reduced in NF- $\kappa \mathrm{B} 1$-deficient macrophages stimulated ex vivo with lipopolysaccharide (LPS), although in vivo relevance of this in vitro effect remains to be determined. In summary, $\mathrm{NF}-\kappa \mathrm{B}$ appears to be at the crossroads of the inflammatory response in atherosclerosis, fine-tuning the response of the vessel wall to injury (Fig. 2).

\section{2. $J N K / A P-1$}

AP-1 (activator protein-1) is a transcription factor consisting of homodimers or heterodimers of Fos (c-Fos, FosB, Fra-1 and Fra2), Jun (c-Jun, JunB, JunD), or ATF subunits which recognize either 12-O-tetradecanoylphorbol-13-acetate (TPA) response elements or cAMP response elements (CRE) (626). Jun proteins can homodimerize, but Fos proteins can only form stable dimers 


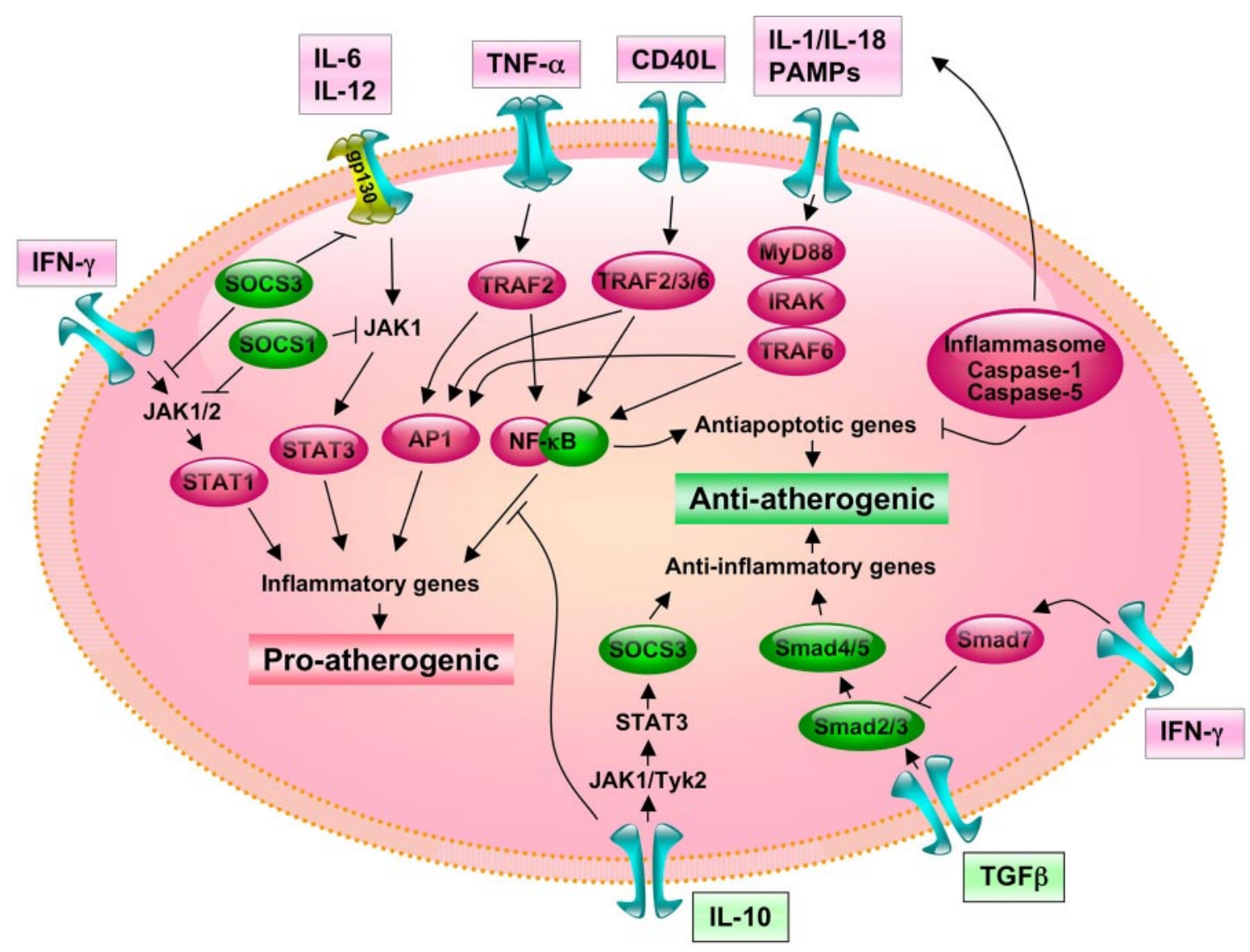

FIG. 2. Cross-talks between proinflammatory/proatherogenic and anti-inflammatory/antiatherogenic signal transduction pathways. Inhibitory Smads such as Smad7 downstream of IFN- $\gamma$ signaling associate with activated receptors and interfere with Smad2 and Smad3 binding. It is noteworthy that like IFN- $\gamma$, the anti-inflammatory cytokine IL-10 also activates JAK and/or STAT proteins. However, the IL-10/IL-10R interaction activates JAK1 and Tyk2, leading to STAT3 and SOCS3 activation, which is central for the anti-inflammatory responses of IL-10 in macrophages. The inflammasome may be a central link between apoptosis and inflammation in pathological conditions. NF- $\kappa \mathrm{B}$ may have a dual role in atherosclerosis, being proatherogenic through its proinflammatory properties, and antiatherogenic through its antiapoptotic activities.

with Jun. Phosphorylation of c-Jun by c-Jun $\mathrm{NH}_{2}$-terminal kinases (JNKs) results in enhanced transcriptional activity of complexes containing AP-1 dimers (734).

JNK belongs to the family of stress-activated protein kinases that also includes the p38 protein kinases. Three highly related but distinct gene products, JNK1, JNK2, and JNK3, can be expressed as a total of 10 isoforms as a result of variable mRNA splicing (259). JNK1 and JNK2 show a broad tissue distribution, whereas JNK3 is expressed predominantly in neurons but also in cardiac smooth muscle and the testes (770). Targeted deletion of the genes coding for JNK1 or JNK2 results in abnormal thymocyte selection (588) and loss of T-lymphocyte differentiation and effector function (179). JNK3 knockout mice show resistance to neuronal apoptosis, directly implicating JNK in at least some specific instances of programmed cell death (678, 768).

JNK phosphorylation is mediated by two MAPK kinases (MAPKKs), MAP2K4 (or MKK4) and MAP2K7 (or MKK7), that they can cooperatively activate JNK (Fig. 1).
Gene disruption studies in mice demonstrate that both MAP2K4 and MAP2K7 are required for full activation of JNK by environmental stressors and that MKK7 is essential for JNK activation by TNF (677).

Many proinflammatory genes, including those encoding TNF- $\alpha$, IL-2, IL-6, E-selectin, ICAM-1, VCAM-1, MCP-1, COX2, and MMPs-1, -9, -12, and -13 (500), are regulated by the JNK pathway, through interaction of AP-1 with other cis-acting sequences in their promoters and with certain transcription factors that bind to these sequences (Fig. 2).

A recent study showed that atherosclerotic lesions were significantly reduced in JNK2-deficient $\mathrm{apoE}^{-/-}$ mice, but not in JNK1-deficient apoE ${ }^{-/-}$mice, compared with apoE $\mathrm{E}^{-1-}$ mice (568). JNK2 expression in leukocytes, rather than in vascular cells, appeared to be responsible for this effect. Indeed, transplantation of $\mathrm{apoE}^{-/-}$ $\mathrm{JNK}^{-/-}$bone marrow into apoE ${ }^{-/-}$mice reduced atherosclerosis to an extent similar to that of $\mathrm{apoE}^{-/-}$ $\mathrm{JNK} 2^{-/-}$mice transplanted with apoE ${ }^{-/-} \mathrm{JNK} 2^{-/-}$bone marrow, whereas $\operatorname{apoE}^{-/-} \mathrm{JNK}^{-/-}$mice transplanted 
with $\operatorname{apoE}^{-/-}$bone marrow showed atherosclerotic lesions equivalent to those of $\operatorname{apoE}^{-/-}$mice transplanted with apoE $\mathrm{E}^{-/-}$bone marrow (568).

\section{JAK/STAT}

The class I and II cytokines induce homodimerization and activation of their cognate receptors, resulting in the activation of associated JAK kinases (JAK1, JAK2, JAK3, and Tyk2) (Table 1) (520a). The activated JAKs phosphorylate the receptor cytoplasmic domains, which creates docking sites for SH2-containing signaling proteins. Among the tyrosine phosphorylated substrates are members of the STAT family of proteins (Table 1) (520a). Receptor engagement and tyrosine phosphorylation activate the cytosolic inactive STATs, resulting in their nuclear translocation and gene activation. This pathway was originally found to be activated by IFNs, but a number of cytokines, growth factors, and hormonal factors also activate JAK and/or STAT proteins (Fig. 1). In particular, IL-6 binds to the IL-6 receptor $\alpha$-chain and gp130, which activate JAK1 and STAT3. IFN- $\gamma$ utilizes JAK1 and JAK2, and usually activates STAT1. It is noteworthy that the anti-inflammatory cytokine IL-10 also activates JAK and/or STAT proteins (reviewed in Ref. 481). The IL-10/ IL-10R interaction activates JAK1 and Tyk2, which are associated with the IL-10R1 and IL-10R2, respectively.

STAT3 can be activated by a number of cytokines, especially those of the IL-6 family, mediating the expression of several acute-phase response genes. Yet, STAT3 appears to play a critical negative role in controlling inflammation, as shown in mice with STAT3 deletion in specific cell types, including keratinocytes (594), T cells (666), macrophages/neutrophils (664), cardiomyocytes (309), or endothelial cells (322), STAT3 deficiency being embryonically lethal. STAT3-deficient T cells show severely impaired IL-6-induced cell proliferation, due to the lack of IL-6-mediated prevention of T-cell apoptosis (666). STAT3 deletion in mice within the macrophage/neutrophil lineage results in chronic inflammation and pathological colitis with age, due to the enhancement of the Th1 response by blockade of IL-10 signaling (664). Removal of STAT3 from hematopoietic progenitors also results in increased proinflammatory cytokine production, inflammatory bowel disease, and an expanded macrophage population (732). Interestingly, STAT3-deficient macrophages and neutrophils show increased production of inflammatory cytokines in response to LPS, which cannot be reduced by IL-10 (664). STAT3 activation by IL-10 is therefore central for anti-inflammatory responses in macrophages and neutrophils (Fig. 2). It is noteworthy that mice with conditional STAT3 deletion in endothelium also show exaggerated inflammation and leukocyte infiltration in multiple organs upon LPS challenge (322). An endothelium-derived soluble factor that is dependent on STAT3 is likely to control IFN- $\gamma$ production during LPS-induced inflammation (322).

In terms of immunoregulation, STAT4 and STAT6 are crucially important for the differentiation of Th cells. IL- 4 activates STAT6 and promotes the differentiation of Th2 cells (634). Conversely, IL-12 activates STAT4 and drives the differentiation of naive $\mathrm{T}$ cells into Th1 cells that produce IFN- $\gamma$ (325). In atherosclerosis, the Th cell response is of the Th1 type, characterized by abundant secretion of IFN- $\gamma$ (264). Yet, Th2 profile does not necessarily offer protection against atherosclerosis and might even be proatherogenic (see sect. vIB3). Therefore, targeting STAT4 and STAT6 could be of use in the treatment of atherosclerosis. Interestingly, statins, which are believed to exert beneficial effects in cardiovascular disease beyond cholesterol lowering (350), have been reported to inhibit Th1-mediated disease and to block activation of STAT4 $(386,492,778)$ and induction of major histocompatibilty complex (MHC)-II expression by IFN- $\gamma$ (366). Other drugs, including rapamycin and lisofylline, have also been reported to block STAT4 activation (127, 771). Interestingly, a recent study showed that rapamycin reduces atherosclerosis in apoE ${ }^{-/-}$mice, with concomitant decreased expression of IL-12p40, IFN- $\gamma$ and IL-10 mRNA, and enhanced expression of TGF- $\beta 1$ (190). Pentoxifylline, a methylxanthine derivative of lisofylline, has been reported to have protective effects against atherosclerosis in apoE $\mathrm{E}^{-/-}$mice, associated with a reduced Th1 polarization of Th lymphocytes (373).

Cytokine signaling by the JAK/STAT pathway is regulated, in part, by a family of endogenous JAK kinase inhibitor proteins termed suppressors of cytokine signaling (SOCS) (748). The SOCS family consists of eight members [SOCS-1 to SOCS-7 and cytokine-inducible SH2 proteins (CIS)] all sharing a central SH2 domain and a $\mathrm{COOH}-$ terminal SOCS box. Both SOCS1 and SOCS3 inhibit JAK tyrosine kinase activity; SOCS1 directly binds to the activation loop of JAKs through the SH2 domain, while SOCS3 binds to cytokine receptors (Fig. 2). SOCS1 regulates INF $\gamma$ signaling, and deficiency leads to lethal disease, which is characterized by exaggerated effects of IFN- $\gamma$. Interestingly, mice lacking both SOCS- 1 and IFN- $\gamma$, though saved from the lethal perinatal syndrome observed in SOCS-1-deficient mice, develop a variety of chronic infections or inflammatory lesions as adults (466). In contrast, SOCS2 regulates growth hormone, and SOCSknockout mice show gigantism. SOCS3 is preferentially expressed in Th2 cells and plays an important role in regulating the onset and maintenance of Th2-mediated allergic immune disease (619).

Very little is known regarding the role of SOCS in atherosclerosis. It has been reported that SOCS-1 inhibits IFN- $\gamma$-induced CD40 expression in macrophages by blocking IFN- $\gamma$-mediated STAT-1 activation, and in so doing suppressing IFN- $\gamma$-induced TNF- $\alpha$ secretion and 
subsequent $\mathrm{NF}-\kappa \mathrm{B}$ activation (733). Inasmuch as the CD40/CD40L pathway actively participates in plaque development and progression (reviewed in Ref. 605), mimics or inducers of SOCS1 might be useful to attenuate the effects of IFN- $\gamma$ in the context of atherosclerosis.

\section{Smads}

TGF- $\beta$-triggered signals are transduced by proteins belonging to the Smad (for vertebrate homologs of Sma and $M a d$ ) family. Smads serve as substrates for TGF- $\beta$ receptors type I and II, in which the cytoplasmic domain possesses serine/threonine kinase activity (453). The type I receptor recognizes and phosphorylates Smad2 and Smad3, which associates with Smad4, forming complexes that participate in DNA binding and recruitment of transcription factors (Fig. 1). Smad3 may also have antagonistic properties, as it plays a critical role in TGF- $\beta$ dependent repression of vascular inflammation by inhibiting AP-1 activity (Fig. 2) (200, 201). In addition to these agonistic Smads, inhibitory Smads (I-Smad) such as Smad6 and Smad7, which associate with activated receptors and interfere with Smad2 and Smad3 binding, are present. Expression of Smad7 is induced by IFN- $\gamma$ as a negative regulator of the TGF- $\beta /$ Smad pathway (684). Recent advances in the study of atherosclerosis point to an important role of TGF- $\beta$ signaling in the protection against excessive plaque inflammation, loss of collagen content, and induction of regulatory immunity (see below and reviews in Refs. 243, 444, 445). Immunohistochemistry and RT-PCR anlaysis of human plaques reveal Smad2, Smad3, and Smad4 expression in macrophages of fibrofatty lesions and in SMC of fibrous caps (320). We also detected phosphorylated Smad2 in the aortic sinus of apoE $\mathrm{E}^{-/-}$mice, indicative of TGF- $\beta$ activity in atherosclerotic lesions (438).

\section{TLR/Myd88 signaling pathways}

At least 10 TLRs (TLR1-10) recognize different PAMPs associated with different classes of pathogens (review in Refs. 303, 665). For example, TLR4 recognizes LPS, which is unique to Gram-negative bacteria, and TLR2 recognizes peptidoglycan found in Gram-positive bacteria. TLR9 recognizes unmethylated CpG motifs, which are abundant in prokaryotic genomes and virus DNA. TLR3 recognizes double-stranded RNA (dsRNA) produced during viral infections. TLRs are characterized by a 150amino acid intracytoplasmic domain named TIR (Toll/IL$1 R / R$ ), which they share with members of the IL-1 receptor (IL-1R) family and plant disease resistance $(\mathrm{R})$ genes, and by an extracellular domain composed of $\mathrm{NH}_{2}$-terminal leucine-rich repeats (LRRs) flanked by characteristic cysteine clusters on the COOH-terminal ( $\mathrm{CF}$ motif) or $\mathrm{NH}_{2}$-terminal (NF motif) side of the LRRs. Upon stimulation, TLRs and the IL-1R family activate the transcription factors NF- $\kappa \mathrm{B}$ and AP-1, leading to production of proinflammatory cytokines. TIR domains play a critical role in TLR signaling. They allow homophilic interactions with the cytoplasmic factor MyD88 that also contains a TIR domain (Fig. 1). MyD88, which is recruited to the receptors after stimulation, contains an $\mathrm{NH}_{2}$-terminal death domain that enables it to bind the death domain-containing serine-threonine kinases of the IL-1R-associated kinases (IRAK) family (reviewed in Ref. 312). As a result, MyD88 functions as an adaptor between receptors of the TLR or IL-1R families and downstream signaling kinases. Following association of MyD88 with IRAK-4, IRAK-4 is autophosphorylated, dissociates from the receptor complex, and interacts with TNF-receptor-associated factor (TRAF)-6. Once activated, TRAF6 activates a heterodimer composed of two ubiquitination proteins called Uev1A and Ubc13, which triggers its association with the MAP3 kinase TAK1 (716). From TAK1, two signaling pathways diverge; one ultimately leads to $\mathrm{NF}-\kappa \mathrm{B}$ activation and the other to MAP kinase activation. Studies using MyD88deficient mice showed that this factor is essential for the NF- $\kappa$ B-dependent induction of TNF- $\alpha$ and IL- 6 in response to TLR agonists (331). Interestingly, analysis of MyD88 mutant mice unexpectedly pointed to the existence of a MyD88-independent pathway downstream of some TLRs. Indeed, TLR4- or TLR3-mediated activation of NF- $\kappa \mathrm{B}$ and AP-1 by LPS and dsRNA, respectively, was not abolished but only delayed in MyD88-deficient mice $(13,331)$.

Recently, enhanced expression of TLR4 was detected in murine (apoE ${ }^{-/-}$mice) and human carotid and coronary atherosclerotic plaques $(186,760)$. TLR1 and TLR2 expression has also been found in human carotid (186) but not in coronary plaques (760). Human epidemiological data demonstrate that an Asp299Gly TLR4 polymorphism, which attenuates receptor signaling, is associated with a decreased risk of atherosclerosis and acute coronary events $(18,64,335)$. Functional TLR4 expression has also been correlated with the development of aortic intimal hyperplasia in a mouse model of artery injury (699), and TLR4 activation by LPS increases atherosclerotic plaque formation in the apoE3*Leiden atherosclerotic mouse model (287).

Interestingly, TLR4 appears to be involved in several aspects of the inflammatory response even in the absence of infection, by recognizing endogenous ligands produced during inflammation. Extracellular matrix components, including the type III repeat extra domain A of fibronectin, low-molecular-weight oligosaccharides of hyaluronic acid, and polysaccharide fragments of heparan sulfate, provoke immunostimulatory responses similar to those induced by LPS, via TLR4 $(315,517,673)$. Moreover, fibrinogen (642) and minimally modified LDL (mmLDL) (471) are able to induce the production of chemokines and cytokines from macrophages through recognition by 
TLR4. Together, these recent findings indicate that TLR4 may exert LPS-independent atherogenic activities (468, 469). Two lines of evidence support this hypothesis: 1) oxLDL enhances TLR4 expression in macrophages (760), and 2) TLR4 or its intracellular adaptor protein, MyD88, reduces atherosclerosis in uninfected apoE-deficient mice, concomitant with a marked reduction in macrophage infiltration and MCP-1 expression in the atherosclerotic lesions (54), and decreased circulating levels of IL-12 and MCP-1 (470).

Remarkably, CD4+CD25+ Treg cells selectively express TLR4-5-7-8 (110). This is of particular importance given the role that Treg cells play in atherosclerosis, as we recently reported $(8,437$; see sect. $\mathrm{vIC})$.

\section{INDUCERS OF CYTOKINE PRODUCTION IN ATHEROSCLEROSIS}

\section{A. Initial Trigger(s)}

According to the classical view of inflammation, cytokines are produced by cells of the innate immune system (monocytes, neutrophils, NKT cells) in response to microbial infection, toxic reagents, trauma, antibodies, or immune complexes (493). In the host, TLRs and intracellular proteins (NOD1 and NOD2, for "nucleotide-binding oligomerization domain") act as sensors of the conserved molecular motifs present on a wide range of different microbes, the PAMPs. Hence, cytokines are secondary mediators of inflammation and not the primary triggers. An etiologic role for infectious agents in atherosclerosis, especially Chlamydia pneumoniae and cytomegalovirus (CMV), has been repeatedly evoked (396) since the first seroepidemiologic evidence of an association of the chlamydia TWAR strain with acute myocardial infarction and chronic coronary disease was reported in 1988 (589). However, the most recent clinical trials, including Weekly Intervention with Zithromax for Atherosclerosis and its Related Disorders (WIZARD) (511), Azithromycin in Acute Coronary Syndrome (AZACS) (114), Antibiotic Therapy After Acute Myocardial Infarction (ANTIBIO) (781), Pravastatin or Atorvastatin Evaluation and Infection Therapy (PROVE-IT) (108), and Azithromycin and Coronary Events Study (ACES) (250), assessing the potential benefits of antibiotic therapy with the goal of targeting Chlamydia pneumoniae showed no effect of treatment in patients with CAD. Moreover, experimental studies showed that infection is not necessary for initiation or progression of atherosclerosis in apoE-deficient mice. Atherosclerosis develops identically in germ-free animals and in animals raised with ambient levels of microbial challenge (749). One must therefore conclude that pathogens do not serve as etiologic agents for atherosclerosis, even though one cannot rule out a role in disease exac- erbation. Several reports indicate that inoculation of atherosclerosis-prone mice with high doses of $C$. pneumoniae fosters atherosclerosis $(292,475)$. Yet, the atherogenic effect of $C$. pneumoniae requires elevated serum cholesterol levels (292).

Atherosclerosis clearly does not develop in any animal model without a significant level of plasma cholesterol, and the dominant role of cholesterol is also well established in humans. While hypertension, diabetes, and smoking are factors that dramatically increase the risk of atherosclerosis, it is not rare to have clinically significant atherosclerosis in the absence of these risk factors. In contrast, below a certain level of cholesterol $(150 \mathrm{mg} / \mathrm{dl})$, atherosclerosis is practically absent in human populations (106), and risk gradually increases with increased plasma cholesterol levels (647). Moreover, primary and secondary clinical trials have established the efficacy of lowering cholesterol with statins for prevention of cardiovascular disease $(256,694)$. It is therefore tempting to hypothesize that the primary trigger of cytokine release in atherosclerosis has a link with cholesterol. Atherogenic cholesterol exists mainly in the form of LDL, which are the main culprit in CAD. In fact, several lines of evidence support the hypothesis that oxidized lipids, including oxLDL, are the most likely triggering factors for cytokine production.

Quantitative analysis of atherosclerosis in fetal aorta showed that fatty streaks are already present at this early stage of life, lesions being more abundant in fetus from hypercholesterolemic mothers than from normocholesterolemic mothers (489). Interestingly, qualitative analysis of lesions depicted similar distribution of native LDL, oxLDL, and macrophages in lesions of offspring from both hypercholesterolemic and normocholesterolemic mothers. The presence of macrophages alone, without native LDL or oxLDL, or their association with native LDL, was almost never observed, and most of the lesions contained both oxLDL and macrophages. A few lesions with native LDL or oxLDL without macrophages were also present. This seminal study allows us to describe the exact chronology of events leading to fatty streak formation in humans, starting with native LDL uptake by the arterial intima, followed by LDL oxidation and, finally, monocyte recruitment after endothelial activation by oxLDL.

C3H mice, which do not develop atherosclerotic lesions either when fed an atherogenic diet or when crossed with the atherosclerosis prone $\mathrm{apoE}^{-/-}$mice, do not respond to in vivo administration of oxLDL, in contrast to C57BL/6 mice (391). Their EC are not activated in the presence of oxLDL, whereas cells from C57BL/6 mice express M-CSF, MCP-1, and VCAM-1 in the same conditions. Yet, C3H EC respond perfectly well to activation by the proinflammatory cytokines IL-1 and TNF- $\alpha(628,629)$. 
oxLDL behaves as a potent inflammatory agent. In vivo administration of oxidized LDL to C57BL/6 mice causes rapid induction of circulating M-CSF and upregulation of genes encoding JE (the murine analog of MCP-1) as well as other inflammatory proteins in various tissues (392). OxLDL stimulates the expression of adhesion molecules on EC (337). OxLDL has chemoattractant activity on monocytes, promotes their differentiation into macrophages, but inhibits their mobility $(555,556)$. Binding of oxLDL to CD36 triggers the release of proinflammatory cytokines in macrophages (310). In addition, incubating human blood mononuclear cells with oxLDL results in T-lymphocyte activation, as assessed by increased expression of IL-2 receptors and HLA-DR antigens on T lymphocytes (215).

Oxidation of LDL generates many "neo-self determinants" that induce an active immune response (288) and may challenge the regulatory pathways responsible for immune homeostasis. Both humoral and cellular immune responses can profoundly affect atherosclerotic development and progression (268).

The amount of lipid retained in macrophages depends on unregulated uptake of oxidized lipoproteins by scavenger receptors, as first identified by Brown and Goldstein (87), counterbalanced by degradation and efflux.

Altogether these findings point to a role of oxLDL as a very early trigger of vascular inflammation. LDL accumulation and modification in the subendothelium trigger monocyte and lymphocyte recruitment. Thereafter, activated macrophages and lymphocytes secrete abundant amounts of cytokines that in turn can activate EC, SMC, and macrophages/lymphocytes to foster cytokine production, leading to a self-perpetuating inflammatory process that becomes less dependent on the presence of oxLDL. This might explain why oxLDL, while instrumental in triggering the early atherosclerotic events, are less critical in upholding the inflammatory environment. This might also explain in part the efficiency of antioxidant therapies in the prevention of atherosclerosis when these therapies are administered at the very beginning of the atherosclerotic process in animal models, but their failure to do so in most secondary or primary prevention clinical trials in humans (reviewed in Ref. 743), where treatment is administered at later stages of the disease when secondary inflammatory mediators become as important as the initial oxidative-related stimulus. It is noteworthy that atherosclerotic plaques do not regress, or regress very slowly, in cholesterol-fed rabbits following short-term withdrawal of cholesterol feeding and normalization of cholesterol plasma levels $(2,155)$. It is only after a prolonged cholesterol withdrawal period that decrease in plaque size, together with reduced vascular inflammation and plaque stabilization, is observed $(7,347,697)$. In humans, aggressive lipid lowering treatment using statins has been shown to be very effective in limiting plaque development and reducing plaque progression (142, 143, $505,506,777)$. The cytokine network may thus serve as a final common proinflammatory pathway regardless of the initiating event and provides a supplemental therapeutic target, especially in late stages of the disease.

Several oxidized lipids and/or phospholipids are lipid bioactive mediators and may serve as primary triggers of the atherosclerotic process. Bioactive lipids have been identified in the atherosclerotic plaque, including the potent inflammatory mediator platelet activating factor (PAF), PAF-like lipids, oxidized phospholipids (oxPL), and lysophosphatidylcholine (lysoPC) (494). Like oxLDL, PAF induces TNF- $\alpha$ production by monocytes $(71,213)$ and MHC class II dependent IFN- $\gamma$ secretion by T lymphocytes (213). Oxidized phospholipids upregulate tissue factor expression in EC (62), as well as in SMC (146). Similarly, lysoPC can enhance IFN- $\gamma$ secretion and gene expression in human T lymphocytes (503) and stimulate the production of IL- $1 \beta$ in macrophages (407). It also stimulates ICAM-1 and VCAM-1 expression $(362,793)$ and induces the release of IL-6 and IL-8 (662) in EC and MCP-1 in EC (663) and SMC (575).

Lipid oxidation products such as lysoPC, 4-hydroxy2-nonenal (4HNE), and oxysterols are contained in oxLDL (662). Oxidized 1-palmitoyl-2-arachidonyl-sn-glycero-3phosphorylcholine (OxPAPC), which is present in minimally modified LDL, is a PAF-like lipid that is found in atherosclerotic plaques. OxPAPC, but not native PAPC, is able to stimulate EC to bind monocytes and to secrete MCP-1, IL-8, and growth-related oncogene (GRO)- $\alpha$ (376, 565,773 ; see review in Ref. 382). Individual lipids identified in OxPAPC include 1-palmitoyl-2-(5-oxovaleroyl)-snglycero-3-phosphorylcholine (POVPC), 1-palmitoyl-2glutaroyl-sn-glycero-3-phosphorylcholine (PGPC), and epoxy-isoprostane-PC $(727,728)$. Oxidized 1-palmitoyl-2linoleoyl-sn-glycero-3-phosphorylcholine (PLPC) also promotes monocyte-endothelial interactions (4). Moreover, epoxyisoprostane and epoxycyclopentenone phospholipids have been identified in OxPAPC that induce MCP-1 and IL-8 in EC (653). Oxidized phospholipids can upregulate tissue factor both in EC (62) and in SMC (146).

Interestingly, it has been shown that OxPAPC inhibits the binding of LPS to LPS-binding protein and CD14, which are required for presenting LPS to TLR-4 (61). It is therefore likely that upon acute bacterial inflammation oxidized phospholipids exert anti-inflammatory properties by inhibiting NF- $\kappa$ B pathway, while under conditions of chronic inflammation, the pro-inflammatory activity of lipid oxidation products becomes more pathologically relevant (382).

Eicosanoids are well-known lipid mediators of inflammation. They comprise a variety of compounds [prostaglandins, thromboxanes, leukotrienes (LT), hydroxy- 
and epoxy-fatty acids, lipoxins, and isoprostanes] that are derived from arachidonic acid. Leukotrienes are a class of eicosanoids that are derived through the action of the 5-lipoxygenase (5-LO). 5-LO is pivotal for the generation of both proinflammatory ( $\mathrm{LTB}_{4}$ and $\mathrm{LTC}_{4}$ ) and anti-inflammatory (lipoxins) mediators. However, in contrast to their inhibitory effects on PMN and eosinophils, lipoxins are potent stimuli for peripheral blood monocytes, stimulating monocyte chemotaxis and adherence (428). Recent biologic and genetic findings implicate the 5-LO pathway in atherosclerosis $(162,279,461,552)$. Mehrabian et al. (461) reported that heterozygotes for the 5-LO gene on the $\mathrm{LDLr}^{-/-}$background had considerably reduced aortic lesions, compared with the advanced lesions observed in $5-\mathrm{LO}^{+/+} \mathrm{LDLr}^{-/-}$mice, despite equivalent hypercholesterolemia. 5-LO pathway also promotes pathogenesis of hyperlipidemia-dependent aortic aneurysm (787). Furthermore, clinical findings showed that variant alleles of 5-LO genes were associated with a significant increase of carotid intima thickness (184). Most recently, a significant association was drawn between the gene encoding 5-LO activating protein (FLAP) and myocardial infarction by analysis of single-nucleotide polymorphism haplotype in humans (279).

Another significant recent discovery is the chemotactic activity of $\mathrm{LTB}_{4}$ on activated CD4 + and CD8 $+\mathrm{T}$ cells expressing the $\mathrm{LTB}_{4}$ receptor, BLT1 $(239,522,661)$. It was found that both Th1- and Th2-polarized CD4+ T cells and antigen-specific CD4 $+\mathrm{T}$ cells, but not naive $\mathrm{T}$ cells, express BLT1 (661). Therefore, the antiatherogenic effects of the blockade of $\operatorname{LTB}_{4} / \mathrm{BLT} 1$ pathway $(6,280)$ might result in part from decreased Th1/Th2 cells recruitment in the plaque.

\section{B. Secondary Triggers}

Once inflammation has been triggered and cytokine release is initiated at the onset of atherosclerotic lesion development, a number of factors that are found in the atherosclerotic plaque can participate in maintaining and amplifying cytokine production (Table 3).

\section{HSP}

Evidence suggests that the inflammatory component of atherosclerosis might, at least in part, involve immune reactivity to heat shock proteins (HSPs) $(446,544,737)$. Animal models of atherosclerosis have shown a very early role for HSP60 in the development of the disease (see review in Ref. 738). HSP60 might be an important autoantigen in atherosclerosis and might play a role similar to that of oxLDL in triggering an autoreactive T-cell response. Rabbits $(757,758)$ or mice $(226)$ immunized with mycobacterial HSP65, which has a high degree of sequence homology with mammalian HSP60, develop en-
TABLE 3. Primary and secondary triggers of cytokine release in atherosclerosis

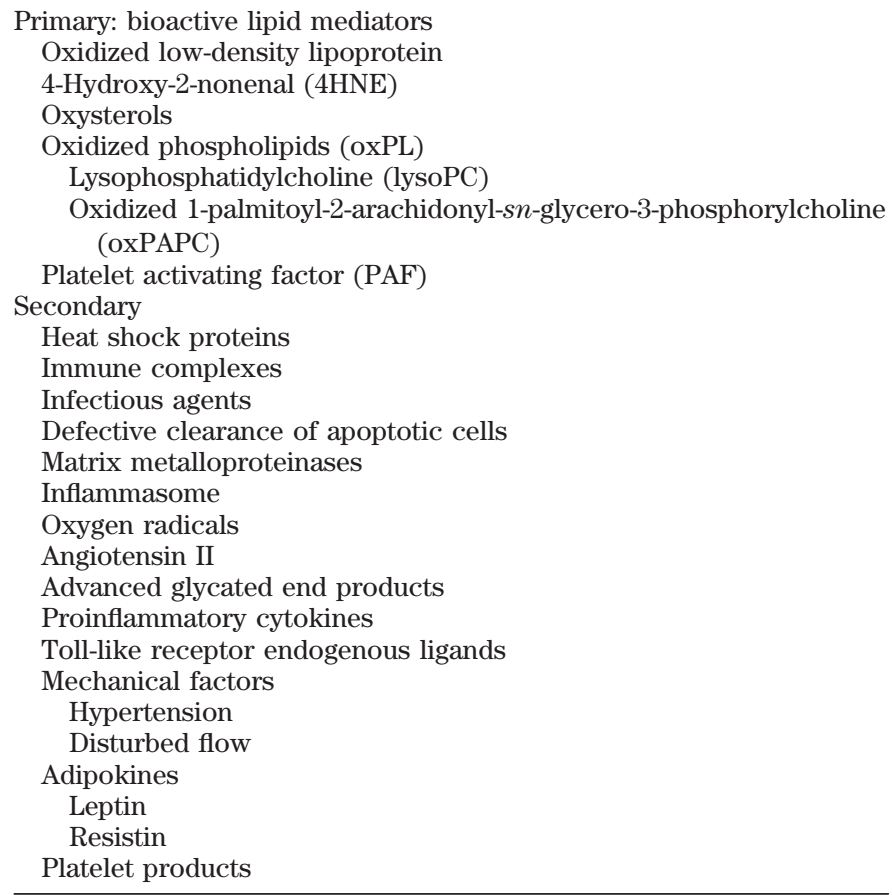

hanced early atherosclerotic lesions. High levels of autoantibodies specific for human HSP60 have been reported to be associated with CAD (792).

Besides their role as autoantigens, HSPs can act as an amplifier of the cytokine production. Although HSPs are typically regarded as intracellular proteins, HSP60 and HSP70 are present in the sera of clinically normal individuals (546, 759), and enhanced levels of circulating HSP60 are associated with early atherosclerosis in clinically normal subjects $(546,759)$, as well as with peripheral vascular disease (749). Elevated HSP60 levels also predict the progression of atherosclerosis in hypertensive patients according to the European Lacidipine Study on Atherosclerosis (ELSA) (545). HSP60, HSP70, HSP90, and gp96 are capable of inducing the production of proinflammatory cytokines by macrophages, and they can stimulate the activation and maturation of dendritic cells as well, via CD14/TLR2 and CD14/TLR4 receptor complex-mediated signal transduction pathways, in a manner similar to the effects of LPS and bacterial lipoprotein (714). In particular, chlamydial and human HSP60 induce TNF- $\alpha$ and MMP production by macrophages (351) and stimulate E-selectin, ICAM-1, and VCAM-1 expression on EC (349). HSP60 also markedly enhances IL-6 production by EC, SMC, and macrophages (349). However, recent evidence suggests that the reported cytokine-inducing effects of HSPs may in part be due to contaminating LPS and LPSassociated molecules (see review in Ref. 680). 


\section{Immune complexes}

OxLDL is a major autoantigen involved in atherosclerosis (reviewed in Ref. 52), and both oxLDL and antioxLDL antibodies are present in atherosclerotic lesions (526, 775). Immune complexes consisting of oxLDL and anti-oxLDL may be ingested by macrophages via $\mathrm{Fc}-\gamma$ receptors, leading to their activation and subsequent release of inflammatory cytokines, oxygen-activated radicals, and MMPs (702). Immune complexes may also induce dendritic cell maturation and the production of immunostimulatory cytokine via ligation of the Fc- $\gamma$ receptors.

\section{Infectious agents}

We have previously indicated why infectious agents are unlikely to be etiological factors in atherogenesis. However, they may participate in exacerbating the inflammatory process associated with atherosclerosis. Chlamydia pneumoniae can infect EC, SMC, and macrophages, resulting in the production of large amounts of chlamydial HSP60 during chronic, persistent chlamydial infections. Chlamydia pneumoniae induces the expression of the adhesion molecules E-selectin, ICAM-1, and VCAM-1 in EC $(329,349)$ and stimulates the production of TNF- $\alpha$, IL-1 $\beta$, IL-6, MMP-2, and MMP-9 in macrophages (330, 349, 351, 499). In addition, Chlamydia pneumoniae is a potent inducer of IL-18 and IFN- $\gamma$ production in peripheral blood mononuclear cells, the latter depending on the release of endogenous IL-18, IL-12, and IL- $1 \beta$ but not on TNF- $\alpha$ (499). Chlamydia pneumoniae-induced synthesis of TNF- $\alpha$ and IL- $1 \beta$ involves TLR2-mediated signals, whereas stimulation of IL-18 production is mediated through MyD88-dependent pathways independent of TLR2 or TLR4 $(498,499)$.

A role for viruses in atherosclerosis was proposed in the 1970s by Catherine Fabricant showing that Marek's disease virus induces atherosclerosis in hypercholesterolemic chickens (196). Other herpes viruses such as herpes simplex virus (HSV) and CMV can contribute to atherosclerosis (90). In the human aorta, EC and SMC appear to be a primary site of infection with CMV, suggesting that the vasculature may serve as a reservoir for CMV (527). Interestingly, the production of IL- 6 and IL- 8 has been shown to be enhanced in CMV-infected SMC and EC (14, 172). Likewise, infection of SMC with human CMV induces strong production of chemokines, RANTES (regulated upon activation, normal T expressed and presumably secreted), and IP-10 (IFN-inducible protein 10) (249). One of the most relevant contributions of herpes viruses to atherosclerosis could be through their potential to initiate the generation of thrombin by having essential phospholipids and $\mathrm{TF}$ activities on their surface (656).

\section{Defective clearance of apoptotic cells}

A variety of mechanisms are involved in apoptotic cell recognition by phagocytes. Innate recognition of nonself involves CD91-calreticulin complex (binding to C1q or to mannose binding lectin which recognizes apoptotic cell-associated molecular patterns by phagocytes), CD14 and $\beta_{2}$-integrins (which bind the inactivated complement fragment iC3b) (reviewed in Ref. 596). Recognition of altered self (oxidized epitopes) is achieved through scavenger receptors, or ligation to bridging proteins, like Gas6 and milk fat globule epidermal growth factor 8 (MFG-E8), which bind Mer kinase receptor and $\alpha_{\mathrm{v}} \beta_{3}$-integrin, respectively, on phagocytes (596). Apoptosis is a mechanism of cell death that does not generate an inflammatory response, since appropriate clearance of apoptotic bodies by professional phagocytes induces the release of the anti-inflammatory cytokines IL-10 and TGF- $\beta$ (596). However, intrinsic defects in the clearance of apoptotic cells are associated with spontaneous and persistent tissue inflammation and autoimmunity. This may be due to reduced production of immunoregulatory cytokines due to defective phagocytosis and/or to the immunogenic and proinflammatory potential of the unremoved apoptotic cells (53). For instance, impaired clearance of apoptotic cells has been described in patients with cystic fibrosis and bronchiectasis (691) and has also been linked to the pathogenesis of systemic lupus erythematosus (SLE) (668). Interestingly, a recent study by Grainger et al. (246) provided evidence that apoE deficiency results in impaired clearance of apoptotic cell debris. This in turn was associated with a systemic increase in proinflammatory markers in apoE ${ }^{-1-}$ mice, including $\mathrm{TNF}-\alpha$ and fibrinogen, which was independent of lipoprotein metabolism (246). With regard to atherosclerotic plaques, we have shown that apoptotic microparticles accumulate in the lipid core (442), most likely as a result of reduced capacities of clearance of apoptotic cells by foam macrophages that are in an oxidant-rich environment (443, 592). Defect in the clearance of apoptotic cells/microparticles may promote and perpetuate proinflammatory cytokine production.

\section{Cellular microparticles}

Microparticles (MPs) are plasma membrane-derived vesicles shed from the plasma membrane of stimulated or apoptotic cells. They are now acknowledged as cellular effectors involved in fundamental physiological processes including intercellular communication, hemostasis, and immunity (reviewed in Refs. 296, 482). MPs are ideal links between inflammation, thrombosis, and atherosclerosis. MPs express a number of proinflammatory and prothrombogenic molecules and could play an important role in the dissemination of these factors to sites remote from the site of their production. MPs are a source for IL- $\beta$ secre- 
tion (427) and are rich in aminophospholipid substrates of secretory phospholipase $\mathrm{A}_{2}$ for the generation of lysophosphatidic acid, a potent proinflammatory mediator and platelet agonist (209). Platelet-derived MPs enhance monocyte arrest on activated endothelium or atherosclerotic carotid arteries, through P-selectin, GPIIb/IIIa, and JAM-A-dependent deposition of RANTES (457, 604), and/or increased endothelial expression of ICAM-1 and monocyte expression of CD11a/CD18 and CD11b/CD18 (36). The induction of monocyte-endothelial adhesion could also be mimicked by arachidonic acid isolated from MPs, suggesting a role for MP-associated bioactive lipids in transcellular communications. These effects of MPs may have contributed to the accelerated atherosclerosis in mice injected with activated platelets (604). P-selectin/ PSGL-1 also enhances the production of leukocyte-derived MP and the recruitment of these MPs to thrombi (reviewed in Ref. 711). In addition, platelet-derived MPs express CD40L, which has been involved in thrombus stabilization (19). Others have shown that leukocyte MP formation was enhanced by inflammatory stimuli (464). Purified leukocyte-derived MPs in turn induced EC IL-6 and IL-8 release, MCP-1, and tissue factor expression (465), suggesting a potential role in the perpetuation of endothelial cell activation. MMPs have been observed in endothelium, platelet, monocyte, and T lymphocyte-derived MPs (177) and may contribute, in conjunction with other factors such as tissue factor, to the proangiogenic potential of these cell fragments. MPs are abundantly present in the lipid core of human atherosclerotic plaques (442) where they are responsible for tissue factor activation and may contribute to plaque inflammation. MPs also circulate at high levels in the peripheral blood of patients with acute coronary syndromes (433) and are suggested to play an important role in endothelial dysfunction (76) in addition to their potential role as carriers of bloodborne tissue factor (231), involved in blood thrombogenicity.

\section{6. $M M P s$}

Several studies have indicated that MMPs can directly or indirectly affect the activity of various cytokines that participate in inflammation and repair processes, including IFN- $\beta$, VEGF, EGF, FGF (see review in Ref. 532 ). Of particular interest in the context of atherosclerosis are the effects of MMPs on TGF- $\beta$, IL- $1 \beta$, and TNF- $\alpha$. Proteolysis of extracellular matrix by MMPs is one of the mechanisms that can release active TGF- $\beta$ from inactive complexes. In both cells and tissue explant models, MMP-3 (429), MMP-9 (779), and MMP-14 (328) have been shown to activate TGF- $\beta$. By activating TGF- $\beta$ in vivo, MMPs would restrain, rather than augment, inflammation. This might, at least in part, account for reduced macro- phage infiltration in atherosclerotic lesions of MMP-3deficient apoE $^{-/-}$mice (637).

IL-1 $\beta$ also requires caspase-1-dependent proteolytic processing for activation. MMP-2, MMP-3, and MMP-9 can cleave and activate the IL- $1 \beta$ precursor (606). Furthermore, beyond activating IL- $1 \beta$, MMP-3 can actually degrade the biologically active cytokine (606), which can also be inactivated in vitro by MMP-1, MMP-2, and MMP-9 (308). Altogether, these data indicate a potential dual role for MMPs in the modulation of cytokine activity, being involved in both activation and inactivation processes.

Similarly, TNF- $\alpha$ is regulated by MMP activity. TNF $\alpha$, which is produced as a $26-\mathrm{kDa}$ membrane-associated protein, is cleaved by TNF- $\alpha$ converting enzyme (TACE) into a soluble $17.5-\mathrm{kDa}$ cytokine. TACE is a member of the disintegrin family of metalloproteinases (ADAM17) (55, 483), and the release of active TNF- $\alpha$ is dramatically reduced in cells derived from ADAM17-deficient mice, indicating that ADAM17 plays a key role as a TNF- $\alpha$ convertase in vivo (788). However, even if ADAM17 is the main modulator of the generation of TNF- $\alpha$ activity, MMP1 and MMP-9 are able to cleave proTNF even though they do not produce active TNF- $\alpha$ (477).

\section{Inflammasome}

Caspases are a family of cysteine proteases that fulfill a critical role in the execution of apoptosis. Moreover, a subfamily of caspases, known as inflammatory caspases, is involved in innate immunity, caspase-1 being the prototypic member. Other members include human caspase -4 and -5 , and mouse caspase- 11 and -12 , all of which contain an $\mathrm{NH}_{2}$-terminal caspase recruitment domain (CARD). Activation of the inflammatory caspases requires the assembly of a unique intracellular complex, designated the inflammasome, that proceeds to cleave and activate IL- $1 \beta$ and IL-18 $(452,681)$. Of note, caspase$1^{-/-}$mice have defects in the production of IL- $1 \beta$ and IL-18 but only subtle defects in apoptotic pathways (358, 390). Evidence is now accumulating that members of the CATERPILLER [CARD, transcription enhancer, R (purine)-binding, pyrin, lots of leucine repeats] gene family, and, in particular, of the NALP subfamily, are important players in this signaling process. When NALP1 is activated by factors that are yet unknown, it interacts with an adaptor protein ASC (apoptosis-associated specklike protein containing a CARD) through homologous pyrin domain (PYD) to induce the assembly of a complex composed of NALP1, ASC, caspase-1, and caspase-5. This brings the caspases in close proximity to each other, thereby inducing their activation (681). Upon activation of caspase- 1 , the $31-\mathrm{kDa}$ IL- $1 \beta$ precursor and the active caspase- 1 colocalize to the inner surface of the cell membrane and caspase- 1 cleaves the precursor (640). The active $17-\mathrm{kDa}$ IL- $1 \beta$ is then released into the extracellular 
compartment. Caspase-1-dependent processing of the 24$\mathrm{kDa}$ IL-18 precursor is believed to be similar to that of IL-1 $\beta$ (176). Interestingly, a single amino acid mutation in the NALP-3 gene has been reported in humans with Muckle-Wells syndrome, a rare autosomal dominant disease characterized by recurrent fevers, neutrophilia, elevated acute-phase proteins and arthritis (3). This mutation in NALP-3 results in a high state of activation of caspase-1 in LPS-stimulated monocytes and increased release of IL- $1 \beta$ compared with cells from patients without the mutation. Inflammasome-related proteins might represent novel pharmacological targets to prevent exaggerated production of IL-1 and/or IL-18, and thereby combat inflammatory diseases.

\section{Oxygen radicals}

Cells present in the atherosclerotic plaque can produce reactive oxygen species (ROS) such as $\mathrm{O}_{2}^{-}, \mathrm{H}_{2} \mathrm{O}_{2}$, and $\cdot \mathrm{OH}$ in response to activation by a number of molecular actors of atherosclerosis, including cytokines (TNF- $\alpha$, IL-1), growth factors (PDGF), vasoactive peptides (angiotensin II), platelet-derived products (thrombin, serotonin), and mechanical factors (cyclic stretch, laminar and oscillatory shear stress) (252). Major sources of ROS include normal products of mitochondrial respiration, NADPH oxidases, NO synthases, cyclooxygenases, lipoxygenases, cytochrome $P-450$ monooxygenase, and xanthine oxidase. These enzymes are all expressed in the atherosclerotic plaque, but evidence suggests that NADPH oxidase-like activity appears to be the major contributing enzymatic source of ROS in the vascular wall, generating superoxide anion in endothelial and smooth muscle cells $(41,253)$. The production of ROS activates reduction-oxidation (redox)-sensitive signaling pathways that regulate inflammatory gene expression.

ROS have been viewed previously as general messengers for signal-induced NF- $\kappa$ B activation (612). However, recent evidence supports the notion that ROS themselves are not direct activators of NF- $\kappa \mathrm{B}$ (273). In fact, ROS may oxidize NF- $\kappa$ B subunits and thus impair the DNA binding and transcriptional activities of NF- $\kappa \mathrm{B}$ (504). At the activation level, the ubiquitination and degradation of NF- $\kappa \mathrm{B}$ inhibitor, I $\mathrm{I} \mathrm{B} \alpha$, is dependent on the kinase activity of IKK complexes. IKK $\alpha$ and IKK $\beta$ contain a redox-sensitive cysteine residue (Cys-179) that may be sensitive to oxidative modification by ROS (582). This would explain the oxidative inactivation of IKK $\beta$ kinase activity observed in cells exposed to ROS (354). In contrast, ROS are potent activators for JNK through oxidative inactivation of the endogenous JNK inhibitors, such as JNK phosphatases and glutathione $S$-transferase (124). Thus ROS may act as unfair brokers between NF- $\kappa \mathrm{B}$ and JNK, inhibiting one but promoting the other, and creating a new form of cross-talk between these two important stress-responsive systems (785).

\section{Angiotensin II}

A large body of evidence indicates that angiotensin II (ANG II) has significant proinflammatory activity in the vascular wall, inducing the production of ROS, inflammatory cytokines, and adhesion molecules. ANG II stimulates ICAM-1 and VCAM-1 expression in EC and SMC $(144,535,553,682)$, as well as E- and P-selectin expression in EC $(15,242)$. ANG II also enhances the functional adhesion of monocytes to EC $(1,336)$ and stimulates MCP-1 production in SMC and monocytes (122, 217, 283, 584). Furthermore, ANG II participates in the vascular synthesis of IL-8 and its homologs macrophage inflammatory protein (MIP)-2 and KC, as well as IP-10 (178, 284, $520,658)$. Interestingly, one study, that needs to be confirmed, reported that ANG II can elicit the synthesis of MCP-1 and RANTES in rat glomerular EC via $\mathrm{AT}_{2}$ rather than $\mathrm{AT}_{1}$ receptors (745).

The proinflammatory effects of ANG II are generally considered to be $\mathrm{AT}_{1}$ receptor dependent and are mediated, at least in part, through NF- $\kappa \mathrm{B}$ and AP-1. A number of studies clearly indicated that inhibition of NF- $\kappa \mathrm{B}$ blocks ANG II-induced expression of inflammatory agents including MCP-1 (283), IL-6 (263), and VCAM-1 (553). $\mathrm{NF}-\kappa \mathrm{B}$ activation appears to be downstream of the $\mathrm{NAD}(\mathrm{P}) \mathrm{H}$ oxidases, inasmuch as antioxidant treatment interferes with its activation by ANG II (163). Moreover, angiotensin IV, a $\mathrm{NH}_{2}$-terminal degradation product of ANG II, has recently been found to activate, via $\mathrm{AT}_{4}$ receptors, the NF- $\kappa \mathrm{B}$ pathway in cultured vascular SMC, and to upregulate proinflammatory genes, such as ICAM-1, IL-6, TNF- $\alpha$, MCP-1, and PAI-1 (195).

In agreement with in vitro observations, in vivo studies clearly indicate that ANG II can stimulate proinflammatory cytokine expression and promote inflammation and atherosclerosis. Chronic infusion of ANG II in apoE $\mathrm{E}^{-1-}$ mice enhances the vascular expression of TNF- $\alpha$, IL- 6 , and IL- $1 \beta$ as well as chemokines and chemokine receptors including RANTES, MCP-1, CCR1 (C-C chemokine receptor 1), CCR2, and CCR3 (501). In addition, ANG II treatment increases atherosclerotic lesion size and promotes unstable plaque phenotype (501). Even though these effects might partly be attributed to ANG II-induced blood pressure elevation, it is noteworthy that blockade of MCP-1, by transfection with a dominant negative of the MCP-1 gene into skeletal muscles, limited ANG II-induced progression and destabilization of atherosclerotic plaques and suppressed the induction of proinflammatory genes (501). Interestingly, genetic disruption of the $\mathrm{AT}_{1}$ receptor in apoE $\mathrm{E}^{-/-}$mice leads to inhibition of atherosclerotic lesion formation, irrespective of blood pressure or plasma cholesterol levels (724), and treatment 
of apoE $\mathrm{E}^{-/-}$mice with an AT1 antagonist inhibits MCP-1 and MIP-1 expression, together with monocyte/macrophage infiltration (178). In humans, it has been shown that elevated plasma levels of MCP-1 in patients with cardiovascular disease are reduced by an ACE inhibitor or an $\mathrm{AT}_{1}$ antagonist $(356,557)$. Remarkably, hypertensive patients display an enhanced ANG II-dependent monocyte activation and adhesion to EC compared with normotensive subjects (180).

Besides its inflammatory effects on vascular cells, ANG II can also modulate adaptive immunity. ANG II triggers the proliferation of splenic lymphocytes through $\mathrm{AT}_{1}$ receptor activation (491) and promotes an immune switch toward a Th1 response. Rats infused with ANG II show an increased IFN- $\gamma$ and decreased IL-4 expression in splenocytes (625). Similarly, splenocytes from hypertensive apoE ${ }^{-1-}$ mice with high ANG II produce more IFN- $\gamma$ than those from hypertensive mice with normal ANG II or normotensive apoE $^{-1-}$ mice (459).

Recently, hyperresponsive cross-linked ANG II-induced $\mathrm{AT}_{1}$ receptor homodimers have been identified, which are covalently bound by the transglutaminase activity of intracellular factor XIIIA (1). High levels of crosslinked $\mathrm{AT}_{1}$ dimers were present on monocytes of hypercholesterolemic apoE-deficient mice, and inhibition of ANG II generation or of intracellular factor XIIIA activity suppressed both the appearance of cross-linked $\mathrm{AT}_{1}$ receptor dimers and symptoms of atherosclerosis. Intriguingly, levels of factor XIIIA activity and $\mathrm{AT}_{1}$ receptor dimers are significantly higher in monocytes derived from hypertensive patients than in monocytes derived from normal subjects and correlate with an enhanced ANG II-dependent monocyte adhesion to EC (1). Furthermore, factor XIIIA activity and the levels of $\mathrm{AT}_{1}$ dimers in hypertensive patients could be reduced or normalized by chronic treatment with an ACE inhibitor. These findings point to a significant contribution of covalent dimerization of $\mathrm{AT}_{1}$ receptors in pathogenic events that drive lesion formation.

\section{AGEs}

Advanced glycation end products (AGEs), the products of nonenzymatic glycation and oxidation of proteins and lipids, accumulate in the vessel wall especially in diabetes but also in euglycemia, in the latter case driven by oxidant stress (767). AGEs may exert their pathogenic effects by engaging cellular binding sites/receptors. A number of cell surface interaction sites for AGEs have been identified, including macrophage scavenger receptors type II, OST-48, 80K-H, galectin-3, CD36, and receptor for AGE (RAGE) (see review in Ref. 767). The interaction of AGEs with macrophages has been shown to activate macrophages in an NF- $\kappa \mathrm{B}$-dependent fashion, leading to the induction of PDGF, insulin-like growth factor (IGF)-I, and proinflammatory cytokines, such as IL- $1 \beta$ and TNF- $\alpha$ (286, 706). Binding of AGEs to endothelial RAGE results in the depletion of cellular antioxidant defense mechanisms (e.g., glutathione, vitamin C) (51) and the generation of ROS (767). As a consequence of the increased cellular oxidative stress, AGE-activated EC express the procoagulant tissue factor and adhesion molecules such as E-selectin, ICAM-1, and VCAM-1 (38, 39, 51, 194).

In addition to AGEs, RAGE is a signal transduction receptor for S100/calgranulins that can also activate EC, SMC, and peripheral blood mononuclear cells (PBMC), including $\mathrm{T}$ cells, and trigger the generation of proinflammatory cytokines and adhesion molecules (286, 767). For example, incubation of EC with EN-RAGE (extracellular newly identified RAGE-binding protein) or S100B also causes VCAM-1 induction (286). Atherosclerotic lesions in diabetic apoE $\mathrm{E}^{-/-}$mice display accelerated accumulation of AGEs and S100/calgranulins and enhanced expression of RAGE compared with nondiabetic apoE ${ }^{-/-}$mice (343). Administration of murine soluble RAGE (sRAGE) suppresses the increased lesion area and complexity associated with diabetes $(92,531)$. In parallel, the treatment induces a reduction in the levels of tissue factor, VCAM-1, AGEs/S100/calgranulins, and nuclear translocation of $\mathrm{NF}-\kappa \mathrm{B}$ in the aorta of sRAGE-treated mice compared with untreated diabetic animals $(343,531)$.

\section{Proinflammatory cytokines}

The fact that cytokines favor their own production is a well-recognized phenomenon. Not only does IL-1 induce IL-1 (175) and TNF- $\alpha$ induce TNF- $\alpha$ (168), but these main directors of the inflammatory process induce a large panel of cytokines and other inflammatory mediators acting in a signaling cascade on target cells, as well as within autocrine loops $(164,720)$. Negative regulatory loops have also been described with IL-10 (170). In the late 1980s and early 1990s, observations made in experimental models of endotoxinemia led to the conclusion that TNF- $\alpha$ was a prerequisite for the induction of many other inflammatory cytokines (see review in Ref. 113). Injection of LPS in experimental animal models or in human volunteers led to the appearance of TNF- $\alpha$ in the bloodstream before any other cytokines could be detected. Moreover, experiments conducted with anti-TNF- $\alpha$ antibodies indicated that blocking TNF- $\alpha$ in bacterial or endotoxin-induced shock models led to a dramatic decrease in the levels of other cytokines measured in the bloodstream. However, while TNF- $\alpha$ may help in perpetuating sustained levels of proinflammatory cytokines, it does not appear to be a prerequisite for their induction in atherosclerosis. Even though the expression of proinflammatory cytokines is significantly decreased in $\operatorname{apoE}^{-/-}$mice deficient in 
TNF- $\alpha$ compared with apoE $\mathrm{E}^{-/-}$mice, it is not totally abolished (515).

Of importance, the immunoinflammatory pathway related to CD40 and its ligand (CD40L) mediates proatherogenic biological responses, such as the expression of cytokines, chemokines, growth factors, matrix metalloproteinases, and procoagulants on EC, SMC, and macrophages, suggesting a major role in atherosclerosis (see sect. $\mathrm{v} A)$.

\section{TLR endogenous agents}

As discussed above, TLRs have been involved in the development of atherosclerosis. Activation of TLRs through PAMPs is a well-recognized pathway that leads to the production of cytokines by macrophages and vascular cells. For instance, LPS-associated TLR4 signaling promotes a proinflammatory phenotype in vascular SMC, inducing the release of MCP-1 and IL-6 and the expression of IL-1 $\alpha$ (769). Dendritic cells are also present in the atherosclerotic plaque (59), and after TLR activation, $\mathrm{CD} 1 \mathrm{~b}+$ dendritic cells secrete high levels of IL-12p40, TNF- $\alpha$, and IFN- $\gamma$, but no IL-10 (355). However, in the context of atherosclerosis, it is important to envision the potential role of TLR activation by nonpathogenic endogenous agents. Accordingly, a recent study reported that mmLDL is able to stimulate early gene and protein expression of TNF- $\alpha$, IL-6, MCP-1, and MIP-2 in macrophages through the TLR4/MyD88, PI3K/Akt, and ERK1/2 pathways, and in the absence of NF- $\kappa \mathrm{B}$ activation (471). Also, ox-PAPC, a bioactive component of oxidized lipoproteins, interacts with TLR4 to induce IL-8 in EC through interaction with CD14 (715).

\section{Mechanical factors}

A) SHEAR STRESS. Blood flow-induced shear stress has long been recognized as critically important in atherogenesis $(111,148)$. Atherosclerotic lesions preferentially develop in areas of disturbed or oscillatory flows, including arterial bifurcations, branch ostia, and curvatures. The vascular endothelium is extremely sensitive to changes in blood flow $(160,380)$; in vitro experiments suggest that physiological levels of shear stress are anti-inflammatory and antiadhesive, while low or oscillatory shear stress promotes oxidative and inflammatory transformations in EC, with enhanced monocyte adhesion, VCAM-1, ICAM-1, and E-selectin expression (116, 478). Flow is able to block TNF-mediated endothelial VCAM-1 expression by inhibiting JNK and p38 MAP kinases (765), a process that involves decreased expression of thioredoxin-interacting protein (766). Also, transcriptional profiling studies identified the Kruppel-like factor (KLF)-2 as a flow-induced anti-inflammatory transcription factor, being inhibited by IL-1 $\beta$ and induced by laminar shear stress in cultured EC
(620). In vivo, lesion-prone areas of disturbed flow show constitutive activation of NF- $\kappa \mathrm{B}(262)$ and a greater propensity for LPS-induced VCAM-1 and E-selectin expression than areas of laminar flow.

B) HYPERTENSION. Epidemiological investigations clearly pointed out that hypertension is a powerful cardiovascular risk factor. Besides being associated with exaggerated atherosclerosis, elevated blood pressure levels have been found to be highly predictive of atherosclerosis-associated cardiovascular events, including ischemic coronary disease, stroke, and peripheral arterial disease (321). In human subjects, carotid artery intima-media thickness, measured with high-resolution B-mode ultrasound, is highly correlated with blood pressure levels and accurately reflects cardiovascular risk (517a). Experimental studies have demonstrated that hypertension increases the rate of atherosclerotic plaque development in hypercholesterolemic rabbits (128), monkeys (755), and, as shown more recently, in mouse models of atherosclerosis (345, 459). Hypertension occurs under several conditions, some linked to the activation of the renin-angiotensin system and characterized by elevated circulating ANG II, some with normal ANG II levels. Interestingly, by using hypertensive apoE ${ }^{-/-}$mice with either elevated plasma ANG II levels (two-kidney, one-clip model of renal hypertension), or normal plasma ANG II levels (one-kidney, one-clip renal hypertension), Mazzolai et al. (459) found that both forms of hypertension led to a similar increase in atherosclerotic plaque size compared with normotensive animals. However, the atherosclerotic plaques of hypertensive animals with high ANG II showed signs of instability, including higher macrophage content, lower collagen and SMC accumulation, and larger lipid core than plaques from hypertensive $\operatorname{apoE}^{-1-}$ mice with normal ANG II, which were of a comparatively more stable phenotype (thicker fibrous cap, less inflammatory cell infiltration, and smaller lipid core). In addition, hypertensive apoE $\mathrm{E}^{-/-}$mice with high ANG II showed enhanced systemic inflammation compared with hypertensive mice with normal ANG II, as evidenced by increased serum IL-6 levels and white blood cell counts.

Several mechanisms can account for hypertensioninduced atherosclerosis. Pressure-induced stretch of the vessel wall increases endothelial permeability to LDL and accentuates LDL accumulation in the intima, which is central to the atherogenic process (467). In addition, hypertension may promote or aggravate vascular inflammation. Indeed, mechanical strain stimulates the expression of ICAM-1 in EC (126) and MCP-1 in SMC (109), which is in good agreement with in vitro studies in organ culture of mouse carotid artery showing that high intraluminal pressure activates NF- $\kappa \mathrm{B}$ (383). Moreover, high blood pressure in vivo upregulates the arterial expression of MCP-1 (109), ICAM-1, and P-selectin (717). 


\section{Adipokines}

A large body of evidence links obesity with accelerated atherosclerosis (460). Adipose tissue is an active endocrine and paracrine organ that releases a large number of cytokines and bioactive mediators, designated adipokines. These products influence not only body weight homeostasis but also inflammation, coagulation, and fibrinolysis, which ultimately affects atherosclerosis and its clinical complications. Adipokines with proinflammatory activities include TNF- $\alpha$, IL-6, plasminogen activator inhibitor-1 (PAI-1), angiotensinogen, leptin, and resistin (372). Increased production of these proteins by adipose tissue in obesity is likely to raise circulating levels of acute-phase proteins and inflammatory cytokines leading to a state of chronic low-grade inflammation that characterizes the obese.

Leptin, which shares structural and functional similarities with the IL-6 family of cytokines (783), enhances the production of TNF- $\alpha$, IL-6, and IL-12 from LPS-stimulated monocytes/macrophages (411). Leptin also plays an important role in the regulation of adaptive immunity. Leptin alters the Th1/Th2 balance in favor of a Th1 response associated with increased IL-2 and IFN- $\gamma$ as well as decreased IL-4 production (414). Moreover, recent observations provided evidence that serum leptin levels are negatively correlated with the percentage of circulating CD4+CD25+ regulatory $\mathrm{T}$ cells in patients with autoimmune disease such as multiple sclerosis (454). This in vivo observation is substantiated by experimental findings showing that the number of Treg is increased in leptindeficient $(o b / o b)$ and leptin receptor-deficient $(d b / d b)$ mice (454). However, ob/ob $\mathrm{LDLr}^{-1-}$ mice have been shown to develop more atherosclerotic lesions than wildtype $\mathrm{LDLr}^{-1-}$ mice $(271,463)$. Yet, leptin deficiency causes marked hypercholesterolemia and lesions of $o b / o b$ $\mathrm{LDLr}^{-1-}$ mice appear to be much smaller than those usually observed in mice of similar age (22-26 wk old) having equivalent cholesterol levels $(\sim 10-12 \mathrm{~g} / \mathrm{l})$ (personal observation). It would be important to examine whether this finding could be attributed to a better regulatory T-cell response in the leptin-deficient mice. This interpretation is consistent with the recent finding that leptin administration enhances atherosclerotic lesion development in apoE $\mathrm{E}^{-/-}$mice (63).

Resistin is another adipokine with potent inflammatory activities. Resistin seems to be expressed at much higher levels in mononuclear leukocytes, macrophages, and bone marrow cells than in human adipose cells (536). Resistin stimulates the production of TNF- $\alpha$, IL-6, and IL-1 in human PBMC (70). It is noteworthy that plasma resistin levels are correlated with markers of inflammation [soluble TNF receptor (sTNFR)-2 and IL-6] and are predictive of coronary atherosclerosis in humans (567). Taken together, these data indicate that leptin and resistin may represent a novel link between metabolic signals, inflammation, and atherosclerosis.

On the contrary, adiponectin exerts potent antiinflammatory properties. It inhibits TNF-induced expression of adhesion molecules in vascular EC (523), blocks lipid accumulation in macrophages, and suppresses the expression of class A scavenger receptors (524). Adiponectin also upregulates the expression of IL-10 in human monocyte-derived macrophages and increases TIMP-1 expression through IL-10 induction (361). Plasma adiponectin levels are reduced in patients with CAD (523), and overexpression of adiponectin in apoE $\mathrm{E}^{-/-}$mice inhibits the progression of atherosclerosis (764), an effect that appears to be mediated by adiponectin-induced IL-10 production (361).

\section{Platelet products and coagulation factors/others}

Thrombin is a serine protease that has a central role in hemostasis and thrombosis. It is generated in the process of activation of the coagulation cascade. Once formed, thrombin cleaves fibrinogen to produce the fibrin mesh of the blood clot. Thrombin also acts on cells through cleavage of specific receptors, which belong to the family of protease-activated receptors (PARs), including PAR-1 and PAR-3. In addition to these procoagulant effects, thrombin participates in inflammation and repair of injured tissues. It stimulates the secretion of other inflammatory mediators. It causes mast cell degranulation and release of histamine (562) and promotes production of IL-1 by activated macrophages (318). Thrombin can also induce, in a PAR-1-dependent way, the expression of E-selectin, ICAM-1, and VCAM-1 and enhances neutrophil and monocyte adhesion to the endothelium $(247,326,327)$. In addition, thrombin stimulates endothelial production of IL-6 (449), IL-8 and MCP-1 (134, 247), and macrophage migration inhibitory factor (MIF) expression (633).

5-Hydroxytryptamine (5-HT), known as serotonin, is a well-characterized neurotransmitter and vasoactive amine. 5-HT is synthesized and released by mast cells, basophils, platelets, and enterochromaffin cells. Enhanced extracellular levels of this amine during inflammation and platelet activation are well documented (456). 5 -HT inhibits TNF- $\alpha$ production but increases the secretion of IL- $1 \beta$, IL-6, IL-12p40, and IL-8 in LPS-stimulated monocytes $(131,183)$.

Mast cells have been reported to likely play a role in the progression of heart failure, atherosclerosis, and plaque rupture of atheroma $(367,537)$. Mast cell tryptase can stimulate the production of MCP-1 and IL-8 (136, 341). 


\section{CYTOKINES AND CYTOKINE RECEPTORS IN HUMAN ATHEROSCLEROTIC PLAQUES}

\section{A. Cytokine Expression in Plaques}

The first evidence that cytokines are expressed in the atherosclerotic plaque stems from the observation in the mid 1980s by Hansson and co-workers (317) that most of the cells present in the plaque express the MHC class II antigen HLA-DR, indicating that IFN- $\gamma$ must be produced in the vicinity of these cells. This was demonstrated later on by the same group $(214,267)$. By the late 1980s, immunohistochemistry, in situ hybridization, or RT-PCR techniques had been used to identify in human atherosclerotic plaques, mainly from carotid endarterectomy specimens, a number of growth factors and cytokines: PDGF (581, 740), TNF- $\alpha$ (26, 35, 585, 676), IL-1 (611), MCP-1 (CCL2) (495, 774), IFN- $\gamma$ (267), and M-CSF (130). Thereafter, as novel cytokines were gradually discovered, their expression in human atherosclerotic plaques was studied and reported (Table 4).

In 1999, Hansson and colleagues (214) determined the expression profiling of Th1 and Th2 cytokines in advanced human atherosclerotic plaques. They found that

TABLE 4. Cytokines expressed in human atherosclerotic plaques

TNF- $\alpha(26,35,585,676)$

IL-1 (611)

IL-2 (214)

IL-3 (mRNA expression) (86)

IL-6 $(586,618)$

IL-8 (CXCL8) $(22,586,718)$

IL-10 (441, 687)

IL-12 (687)

IL-15 (289, 750)

IL-18 $(228,435)$

IFN- $\gamma(214,267)$

Oncostatin M (476)

TGF- $\beta 1$, TGF- $\beta 2$, TGF- $\beta 3(58,214)$

Activin A, a member of the TGF- $\beta$ superfamily (191)

Cyclophilin A, a potent chemoattractant for leukocytes (140, 314) M-CSF (130)

\section{Chemokines}

MCP-1 (CCL2) (495, 774)

IFN- $\gamma$-inducible CXC chemokines

Monokine induced by IFN- $\gamma$ (Mig/CXCL9) (424)

IFN-inducible protein 10 (IP-10/CXCL10) (424)

IFN-inducible T-cell $\alpha$-chemoattractant (I-TAC/CXCL11) (424)

CXCL16/SR-PSOX (751)

MIP-1 $\alpha$ (CCL3) $(274,739)$

RANTES (CCL5) (274, 739)

Fractalkine (CX3CL1), the ligand of CX3CR1 (746)

$$
\text { Bone-associated cytokines }
$$

Osteopontin (OPN) $(230,508)$

Osteoprotegerin (OPG) (173)

Receptor activator of NF- $\kappa$ B ligand (RANKL) (238)

Reference numbers are given in parentheses.
IL-2 was present in $50 \%$ of plaques, and IFN- $\gamma$ was detected in some but not all of the IL-2-positive plaques. In contrast, the expression of IL- 4 and IL-5, Th2 cytokines, and TNF- $\beta$ (lymphotoxin- $\alpha$ ), expressed by both Th1 and Th2 cells, was very scarce, and IL-10 was not detected at all. However, we (441) and others (687) have found that IL-10 is produced in atherosclerotic lesions and correlates with diminished expression of inflammatory mediators. TGF- $\beta$ was expressed abundantly in all plaques as previously reported (502). Comparisons between sections stained for TGF- $\beta$ and for cell type-specific antigens implied that macrophages, T cells, and smooth muscle cells expressed this group of cytokines. Among isoforms, TGF- $\beta 2$ was detected in high frequency and exhibited stronger intensity of staining than TGF- $\beta 1$ or TGF- $\beta 3$. The distribution of TGF- $\beta$ overlapped with that of its transport protein, LTBP. This suggested that TGF- $\beta$ is actively secreted. Subsequent experiments in murine models of atherosclerosis analyzing the role of IL-10 $(104,434,542)$ and TGF- $\beta(236,245,420,572)$ in this setting led to the conclusion that the balance between proinflammatory and anti-inflammatory cytokines is decisive for disease development and progression.

A group of noncollagenous matrix proteins originally identified as important in bone mineralization, including osteopontin (OPN), osteoprotegerin (OPG), and receptor activator of NF- $\kappa \mathrm{B}$ ligand (RANKL), are expressed by macrophages, EC, and SMC in plaques $(173,230,238$, 508). They have pleiotropic effects that influence matrix turnover, cell migration, and inflammation (248, 609). OPN and OPG expression is greater in symptomatic than in asymptomatic carotid atherosclerotic plaques, whereas RANKL expression is similar (238). Remarkably, OPN, also called early T-lymphocyte activation protein-1 (Eta1 ), is needed for Th1 responses and promotes IL-12 expression (29).

Better understanding of the time course of cytokine gene expression is important for successful prevention of plaque development and progression. However, this information cannot be easily obtained in humans. A recent study has addressed this issue in $\operatorname{apoE}^{-/-}$mice (695). After $4 \mathrm{wk}$ of a cholesterol-rich diet, the expression of the proinflammatory cytokines was much more pronounced than anti-inflammatory cytokines. This imbalance between pro- and anti-inflammatory cytokines might account for the progression of atherosclerosis.

\section{B. Cellular Sources of Cytokines}

\section{Vascular cells}

All cells present in the atherosclerotic plaques are potentially able to elaborate a set of cytokines (Table 5; Fig. 3). By the end of the 1980s, Peter Libby and his group identified for the first time the capacity of human vascular 
TABLE 5. Cellular sources of cytokines with potential activities in atherosclerosis

\begin{tabular}{|c|c|c|c|}
\hline Cytokine & Cell Source & Cell Target & Function \\
\hline $\mathrm{IL}-1 \alpha$ & $\begin{array}{l}\text { Macrophages, lymphocytes, EC, } \\
\text { SMC }\end{array}$ & Many cell types & Proinflammatory, stimulates endothelial and SMC activation \\
\hline \multicolumn{4}{|c|}{ 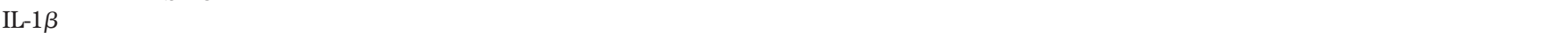 } \\
\hline IL-2 & Activated T cells & $\begin{array}{l}\text { Macrophages, T \& B cells, NK } \\
\text { cells }\end{array}$ & $\begin{array}{l}\text { T-cell growth factor, stimulates NK activity, stimulates Treg } \\
\text { cells }\end{array}$ \\
\hline$I L-3$ & $T$ cells, mast cells & $\begin{array}{l}\text { Mast cells, hematopoietic } \\
\text { progenitors }\end{array}$ & $\begin{array}{l}\text { Promotes proliferation and differentiation of mast cell } \\
\text { and hematopoietic cell lineages (granulocytic, } \\
\text { monocytic, megakaryocytic) }\end{array}$ \\
\hline IL-4 & Th2 cells, mast cells & $\begin{array}{l}\text { T \& B cells, mast cells, } \\
\text { macrophages, hematopoietic } \\
\text { progenitors }\end{array}$ & $\begin{array}{l}\text { Proliferation and differentiation of B cells (Ig switching to } \\
\text { IgG }_{1} \text { and IgE) and Th2 cells (anti-inflammatory by } \\
\text { inhibiting Th1 immune responses); stimulates VCAM-1 }\end{array}$ \\
\hline IL-5 & $\mathrm{T}$ cells, mast cells, EC & B cells & $\begin{array}{l}\text { Stimulates growth and differentiation of B cells, Ig } \\
\text { switching }\end{array}$ \\
\hline IL-6 & Macrophages, EC, SMC, T cells & $\begin{array}{l}\text { T \& B cells, hepatocytes, EC, } \\
\text { SMC }\end{array}$ & $\begin{array}{l}\text { Differentiation of myeloid cells, induction of acute phase } \\
\text { proteins, SMC proliferation }\end{array}$ \\
\hline$I L-7$ & Platelets & Monocytes, $T \& B$ cells & Proinflammatory \\
\hline IL-8 & Monocytes, EC, T cells & Neutrophils, T cells, monocytes & Proinflammatory, promotes leukocyte arrest \\
\hline IL-9 & Th2 cells & $\begin{array}{l}\text { T cells, B cells, mast cells, } \\
\text { eosinophils, neutrophils, and } \\
\text { epithelial cells }\end{array}$ & $\begin{array}{l}\text { Promotes proliferation and differentiation of mast cells, } \\
\text { stimulates IgE production, inhibits monocyte activation, } \\
\text { stimulates TGF- } \beta \text { in monocytes }\end{array}$ \\
\hline IL-10 & $\begin{array}{l}\text { Macrophages, Th2, Treg and B } \\
\text { cells, mast cells }\end{array}$ & Macrophages, T \& B cells & $\begin{array}{l}\text { Anti-inflammatory, inhibits Th1 responses, promotes } \\
\text { proliferation and differentiation of regulatory } \mathrm{T} \text { cells }\end{array}$ \\
\hline$I L-11$ & $E C$ & Hematopoietic progenitor cells & Hematopoiesis \\
\hline IL-12 & Th1 cells & $\mathrm{T}$ cells, macrophages & $\begin{array}{l}\text { Proinflammatory; promotes NK and cytotoxic lymphocyte } \\
\text { activity; induces IFN- } \gamma\end{array}$ \\
\hline$I L-13$ & Th2 cells & $B$ cells & Activation of Ig transcription \\
\hline$I L-14$ & EC, lymphocytes & $B$ cells & $B$-cell growth factor \\
\hline$I L-15$ & $E C$, macrophages & $\begin{array}{l}T \& B \text { cells, NK cells, } \\
\text { monocytes }\end{array}$ & $\begin{array}{l}\text { Enhances neutrophil chemokine production, cytoskeletal } \\
\quad \text { rearrangements, phagocytosis; delays apoptosis }\end{array}$ \\
\hline$I L-16$ & $\begin{array}{l}\text { Mast cells, } C D 4^{+} \text {and } C D 8^{+} \\
\quad \text { cells }\end{array}$ & $C D 4^{+}$ & $\begin{array}{l}C D 4^{+} \text {T-cell growth factor; proinflammatory; enhances } \\
\text { lymphocyte chemotaxis, adhesion molecule, and IL-2 } \\
\text { receptor and HLA-DR expression }\end{array}$ \\
\hline$I L-17$ & $\begin{array}{l}\text { Human memory } T \text { cells, mouse } \\
\alpha \beta T C R^{+} C D 4^{-} C D 8^{-} \\
\quad \text { thymocytes }\end{array}$ & $\begin{array}{l}\text { Fibroblasts, keratinocytes, } \\
\text { epithelial and } E C\end{array}$ & $\begin{array}{l}\text { Secretion of } I L-6, I L-8, P G E_{2}, M C P-1 \text { and } G-C S F, \text { induces } \\
\quad I C A M-1 \text { expression, T-cell proliferation }\end{array}$ \\
\hline IL-18 & Macrophages & $\begin{array}{l}\text { T cells; NK cells; myeloid, } \\
\text { monocytic, erythroid, and } \\
\text { megakaryocytic cell lineages }\end{array}$ & $\begin{array}{l}\text { Proinflammatory, induces IFN- } \gamma \text { and other Th1 cytokines, } \\
\text { promotes Th1 development and NK activity }\end{array}$ \\
\hline$G M-C S F$ & Macrophages, EC, lymphocytes & $\begin{array}{l}\text { Hematopoietic stem cells, } \\
\text { neutrophils, macrophages }\end{array}$ & Growth and differentiation of granulocytes, macrophages \\
\hline M-CSF & Macrophages, EC, lymphocytes & $\begin{array}{l}\text { Hematopoietic stem cells, } \\
\text { neutrophils, macrophages }\end{array}$ & Growth and differentiation of macrophages \\
\hline $\mathrm{TNF}-\alpha$ & $\begin{array}{l}\text { Macrophages, T \& B cells, NK } \\
\text { cells, SMC }\end{array}$ & Many cell types & $\begin{array}{l}\text { Proinflammatory, fever, neutrophil activation, bone } \\
\text { resorption, anticoagulant, tumor necrosis }\end{array}$ \\
\hline TGF- $\beta$ & $\begin{array}{l}\text { Platelets, macrophages, Th3, } \\
\text { Treg \& B cells, SMC }\end{array}$ & Many cell types & $\begin{array}{l}\text { Anti-inflammatory; profibrotic; promotes wound healing, } \\
\text { angiogenesis; suppresses Th1 \& Th2 immune responses }\end{array}$ \\
\hline $\mathrm{IFN}-\gamma$ & Th1 cells, NK cells, SMC (?) & $\begin{array}{l}\text { Macrophages, lymphocytes, NK } \\
\text { cells, EC, SMC }\end{array}$ & $\begin{array}{l}\text { Proinflammatory, promotes Th1 immune } \\
\text { responses/secretion of Th1-associated cytokines, inhibits } \\
\text { extracellular matrix synthesis by SMC }\end{array}$ \\
\hline CD40L & $\begin{array}{l}\text { Platelets, T cells, NK cells, EC, } \\
\text { SMC }\end{array}$ & $\begin{array}{l}\text { Macrophages, lymphocytes, NK } \\
\text { cells, EC, SMC }\end{array}$ & $\begin{array}{l}\text { Proinflammatory, promotes Th1 immune } \\
\text { responses/secretion of Th1-associated cytokines, } \\
\text { stimulates MMP secretion }\end{array}$ \\
\hline
\end{tabular}

Cytokines in italics have a yet unknown role in atherosclerosis.

cells to be both source and target of cytokines, showing that IL- $1 \alpha$ and IL- $1 \beta$ induced IL- $1 \beta$ production by human SMC and EC $(721,722)$ and that TNF- $\alpha$ induced TNF- $\alpha$ production by SMC (723). Of note, TNF- $\alpha$ is not expressed by EC. IL-6 is constitutively released by SMC in culture, and its production can rise to the point of representing $\sim 4 \%$ of newly synthetized proteins in activated cells (413). The proinflammatory repertoire of vascular cells includes TNF- $\alpha$, IL-1 $\beta$, IL-6, IL-8, and IL-15 while the anti- inflammatory repertoire is represented predominantly by TGF- $\beta$. EC also express low levels of IL-1ra (171). Vascular cells do not seem to be able to express the antiinflammatory cytokines IL-10, IL-4, or IL-13. Moreover, the response of EC to IL-4 and IL-13 favors inflammation, with sustained expression of VCAM-1 $(85,601,602)$ and Pselectin (772).

EC are also important sources of hematopoietic growth factors including stem cell factor (SCF), IL-3, 


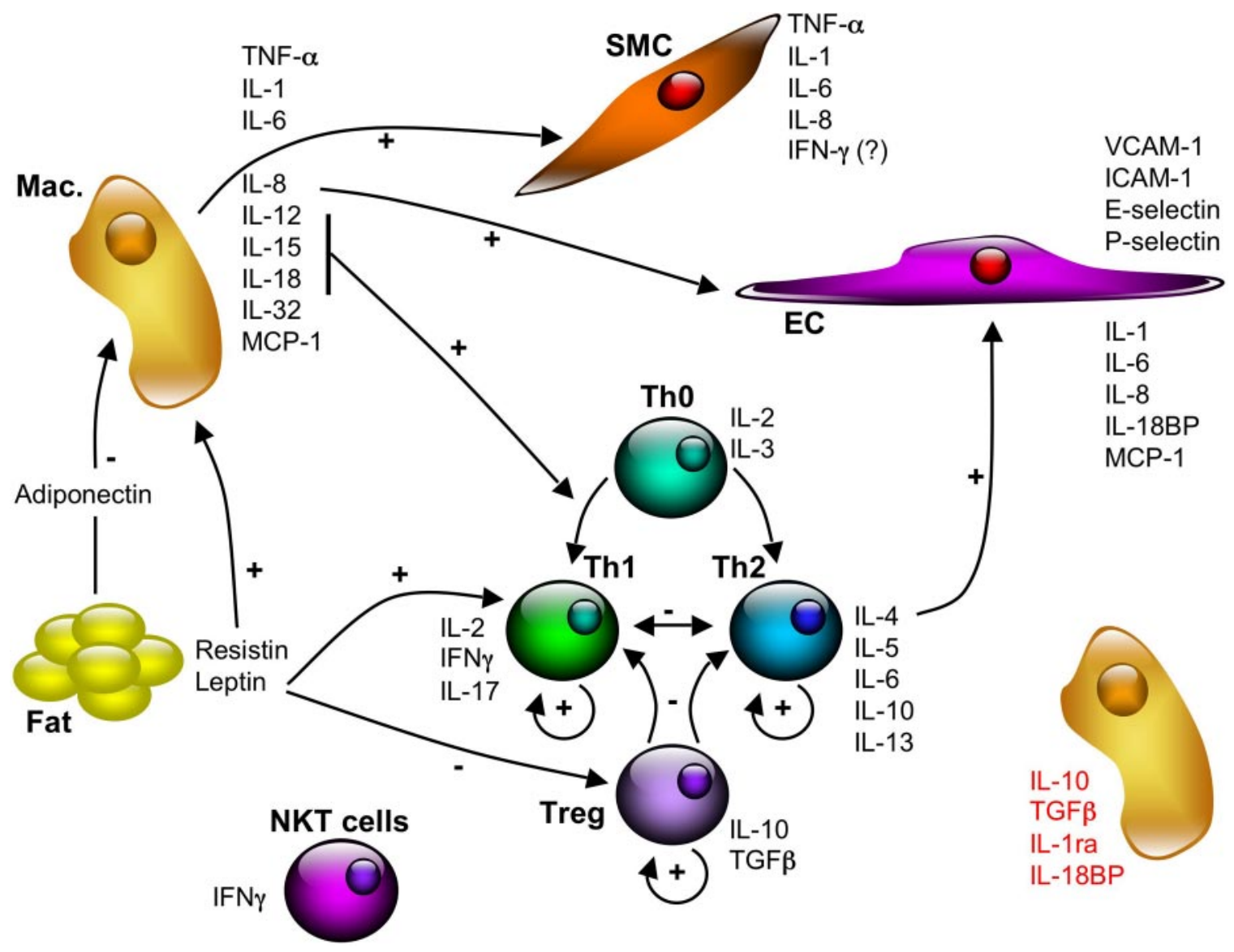

FIG. 3. Cytokines involved in atherogenesis. Cytokines are produced by several cell types, including inflammatory and vascular cells, as well as adipocytes. IL-12 and IL-18 produced by macrophages are potent inducers of IFN- $\gamma$ and promote the differentiation of naive T cells into proatherogenic Th1 cells. Macrophage or macrophage-derived cytokines also activate smooth muscle cells (SMC) and endothelial cells (EC) to produce an array of proinflammatory mediators. On the other hand, the anti-inflammatory cytokines IL-10 and TGF- $\beta$, also produced by macrophages, promote antiatherogenic Treg cell differentiation. Other anti-inflammatory mediators with potent antiatherogenic properties include IL-1 receptor antagonist (IL-1ra) and IL-18 binding protein (IL-18BP). Interestingly, IL-4, the prototype of Th2 cells, has proinflammatory properties on EC. Adipocytes produce both pro- and anti-inflammatory mediators. Leptin activates Th1 cells but inhibits Treg cell function. Adiponectin has been shown to dampen macrophage activation.

GM-CSF, G-CSF, and M-CSF (447). IL-15, a cytokine implied in T-cell migration, has been shown to be produced by EC in response to IFN- $\gamma$ (519). In addition, EC express the chemokines I-309/CCL1, MCP-1/CCL2, MCP-4/CCL13, monokine induced by IFN- $\gamma$ (Mig)/CXCL9, IFN-inducible T-cell chemoattractant (I-TAC)/CXCL11, and MIF (reviewed in Ref. 729). Chemokines produced by SMC include MCP-1/CCL2, Eotaxin/CCL11, Mig/CXCL9, stromal cell-derived factor (SDF)-1/CXCL12, (scavenger receptor that binds phosphatidylserine and oxidized lipoprotein) SR-PSOX/CXCL16, Fractalkine/CX3CL1, and MIF (reviewed in Ref. 729). SMC constitutively express mRNA for the chemokine receptors CCR1 and CCR2, but not CCR3, CCR4, CCR5, or CXCR1 (C-X-C chemokine receptor 1) or CXCR2 (274).

\section{Leukocytes}

Macrophages are certainly the main source of cytokines in the atherosclerotic plaque. Their repertoire is huge, including the proinflammatory cytokines TNF- $\alpha$, IL-1, IL-6, IL-12, IL-15, and IL-18, as well as the antiinflammatory cytokines IL-10 and TGF- $\beta$. IL-32, a recently discovered cytokine that activates typical cytokine signal pathways of NF- $\kappa$ B and p38 MAP kinase (338), can be added to this list. Macrophages have also been reported to produce IFN- $\gamma$ after stimulation by a combination of IL-12 and IL-18, suggesting the possibility of an autocrine activation loop in macrophages (484). However, these results were recently questioned by Schleicher et al. (600) who showed that conventional techniques used to generate peritoneal and bone marrow-derived macrophages in fact contain small quantities of natural killer (NK) cells or CD8 + T cells, respectively, that are fully responsible for the production of IFN- $\gamma$. In the atherosclerotic plaque, IFN- $\gamma$ can be produced by CD $4+$ Th1 cells, CD8 + T cells, and NKT cells.

Macrophages express a number of chemokines: MCP1/CCL2, MCP-4/CCL13, IL-8/CXCL8, GRO- $\alpha$ /KC/CXCL1, Mig/ 
CXCL9, I-TAC/CXCL11, SDF1/CXCL12, SR-PSOX/CXCL16, and MIF, whereas lymphocytes express RANTES/CCL5, MIP1 $\alpha /$ CCL3, MIP $\beta /$ CCL4 (reviewed in Ref. 729 ). It is noteworthy that chemokines may share a close evolutionary relationship with scavenger receptors in that chemokines generally have scavenger receptor-like activity, binding oxLDL through their receptor-binding domain (631). This is especially the case for the transmembrane protein SR-PSOX that is identical to the chemokine CXCL16 $(455,630)$.

A variety of chemokine receptors have been found to be differentially associated with Th cell subpopulations. CCR5 and, to a lesser degree CXCR3, are preferentially found on Th1 cells, whereas CCR4 is preferentially found on Th2 cells (105). CCR4 and CCR8 seem to be specifically expressed by CD4+CD25+ Treg cells $(211,300)$.

\section{Platelets}

Platelets have inflammatory actions and are a rich source of chemokines, cytokines, and growth factors. These factors are preformed and packaged in storage granules and, when released, may participate in atherosclerosis. While IL- $1 \beta$ cannot be detected in resting platelets, it is shed in its active form in microvesicles, after activation with thrombin (402). Platelets are the main source of circulating CD40L (551), and following ligation with the CD40 receptor might be involved in inflammatory cellular cross-talks (281).

From their $\alpha$-granules, platelets secrete CXC chemokines, such as platelet factor 4 (PF4/CXCL4) or epithelial cell-derived neutrophil-activating peptide (ENA-78/ CXCL5), and precursors for the CXCR2 ligand neutrophil attracting peptide (NAP)-2 (CXCL7), such as CTAP-III or $\beta$-thromboglobulin, as well as CC chemokines, such as MIP-1 or RANTES (see review in Ref. 729). The deposition and immobilization of platelet-derived RANTES have been shown to trigger enhanced recruitment of monocytes on activated aortic endothelium $(604,710)$. RANTES was revealed on the luminal surface of carotid arteries with early atherosclerotic lesion in $\mathrm{apoE}^{-1-}$ mice (710). In fact, activated platelets can deliver RANTES and PF4 to the endothelial lining of early atherosclerotic lesions, as well as to the surface of monocytes via a mechanism involving the platelet P-selectin $(298,604)$. The important observation that the intermittent injection of activated, but not P-selectin-deficient, platelets exacerbates lesion formation in apoE $\mathrm{E}^{-/-}$mice strongly suggests that mechanisms of P-selectin-mediated chemokine delivery participate in the in vivo pathogenesis of native atherosclerosis (298).

\section{Mast cells}

Mast cells are inflammatory cells best known for their pivotal role in allergic diseases. They are also present in the arterial wall, where they form part of the inflammatory cell infiltrate and may contribute to atherosclerosis $(319,384,404)$. Mast cells might be an additional source of inflammatory cytokines within the plaque. They are able to produce copious amounts of presynthesized TNF- $\alpha$ within their granules, in addition to de novo synthesis and secretion of TNF- $\alpha$ following stimulation (240).

\section{Biological Effects of Cytokines}

\section{Effects on endothelial permeability}

Alteration of endothelial permeability is an important feature during inflammatory conditions and is associated with leukocyte transendothelial migration and accumulation within the tissues (see Table 6). The intercellular junction complex and its interactions with the cytoskeleton are important for the maintenance of endothelial permeability (43). A number of proinflammatory cytokines, such as TNF- $\alpha$ and IFN- $\gamma$, have been shown to alter the distribution of adhesion receptors involved in cell-cell adhesion, namely, vascular endothelial (VE)-cadherincatenin complexes, and prevent the formation of F-actin stress fibers (744). This results in restructuring of the intercellular junction leading to loss of endothelial permeability and favoring leukocyte transmigration. Complete alteration in intercellular junction organization during inflammatory conditions may require the interplay between inflammatory cell adhesion and secretion of proinflammatory mediators (185).

\section{Activation of adhesion molecule and chemokine expression}

Since the initial discovery that cytokines induce ELAM and VCAM-1 expression on EC (47, 150), many

TABLE 6. Biological effects of cytokines

Effects on endothelial permeability $(185,744)$

Activation of adhesion molecule expression (47, 150)

Induction of chemokine release (424)

Modulation of scavenger receptor expression (291, 363, 388)

Modulation of lipid metabolism $(472,751)$

Activation of 15-lipoxygenase expression in cultured macrophages (621, 754, 756)

Effect of SMC migration/proliferation (266, 267, 400, 670, 723, 776)

Regulation of immune response (Th1/Th2/Treg) $(34,145,431)$

Conversion of $\mathrm{CD} 4^{+}$naive $\mathrm{T}$ cells into $\mathrm{CD} 4^{+}$regulatory $\mathrm{T}$ cells $(120$, 207, 251, 707)

Oxidation of LDL (induction cell oxidant stress) (204, 458)

Stimulation of MMP expression (500)

Modulation of extracellular matrix expression (17)

Modulation of endothelial of SMC progenitor differentiation (370, 389, 686)

Regulation of neovessel formation $(63,69,192,423)$

Promotion of intraplaque neovascularization (705)

Induction of apoptosis (79, 80, 222, 223)

Stimulation of microparticle release (464)

Modulation of endothelial procoagulant activity $(193,216,451)$

Modulation of fibrinolysis (PAI-1) (597) 
cytokines, including, IL- 1 , TNF- $\alpha$, and IFN- $\gamma$, have been implicated in the induction of an array of adhesion molecules and chemokines in the vascular wall. IL-1 and TNF- $\alpha$ stimulate membrane expression of leukocyte adhesion molecules ICAM-1, ICAM-2, VCAM-1, E-selectin, and P-selectin by EC. These molecules interact with specific ligands expressed by neutrophils, lymphocytes, and circulating monocytes. VCAM-1 plays an important role in atherogenesis (151). It selectively promotes the adhesion of mononuclear cells on the vascular endothelium that constitutively express its ligand very late antigen (VLA)-4. VCAM-1 is present in human advanced coronary atherosclerotic lesions but is barely expressed by EC being more prevalent in intimal SMC and plaque microvessels (397, $509,510)$. Cytokines also play an important role in the induction of chemokines by vascular cells, particularly, IL-8 and MCP-1 that are involved in monocyte adhesion and migration into the inflamed vessel wall in atherosclerosis. Other chemokines, such as IP-10, Mig, and I-TAC, are IFN- $\gamma$ inducible and potently chemoattract activated T lymphocytes. These chemokines are expressed in atherosclerotic plaques (424) and may play an important role in T-lymphocyte infiltration and activation in atherosclerosis.

\section{Modulation of scavenger receptor expression and lipid metabolism}

Proinflammatory cytokines have contrasting effects on the expression of the various scavenger receptors. Lectin-like oxidized LDL receptor (LOX)-1, which is detectable in EC, intimal macrophages, and SMC of advanced atherosclerotic plaques, can be induced by proinflammatory stimuli such as TNF- $\alpha$, in addition to its induction by oxidized LDL and other stimuli (363). TNF- $\alpha$ and IFN- $\gamma$ have been shown to increase scavenger receptor expression and function in cultured rabbit aortic SMC (388). However, TNF- $\alpha$ and IFN- $\gamma$ appear to inhibit scavenger receptor SR-A surface expression in macrophages, principally by destabilization of scavenger receptor mRNA (291). This effect of TNF- $\alpha$ may account for the increased scavenger receptor activity, as assessed by acetylated LDL uptake by peritoneal macrophages, in mice deficient for TNF p55 receptors (613). Regarding IFN- $\gamma$, recent studies confirmed and extended its role in lipid metabolism. IFN- $\gamma$ induced foam cell formation through upregulation of SR-PSOX (751), the scavenger receptor for phosphatidylserine and oxLDL, which has been involved in Ox-LDL uptake and subsequent foam cell transformation in macrophages (472). Interestingly, SRPSOX is identical to the chemokine, CXCL16 that ligates CXCR6, expressed in intimal macrophages of human atherosclerotic plaques (751). IFN- $\gamma$ inhibits apoE (81) and the ATP-binding cassette transporter A1 (ABCA1) (530), resulting in decreased cholesterol efflux from macro- phages (530). Thus IFN- $\gamma$ could serve as a molecular link between lipid metabolism and immune activity (751). In contrast, TGF- $\beta$ enhances cholesterol efflux through upregulation of ABCA1 (27) and apoE (794). NF- $\kappa$ B activity has also been reported to affect scavenger receptor expression and function, as suggested by studies in p50 (NF- $\kappa \mathrm{B} 1$ )-deficient mice showing reduced uptake of oxLDL in macrophages from these mice, associated with a reduction in the expression of SR-A (323). IL-4 and IL-13 are activators of 15-LO expression in cultured macrophages (621) through phosphorylation of protein kinase C (PKC) $\delta$ and p38 MAPK acting on STAT1 and STAT3 (754, 756), which may affect lipid oxidation. IL-4 augments acetylated LDL-induced cholesterol esterification in macrophages through SR-A (141).

Interestingly, adipokines may significantly modulate scavenger receptor expression. Treatment of $\mathrm{apoE}^{-1-}$ mice with adiponectin (516) or overexpression of globular adiponectin in apoE $\mathrm{E}^{-1-}$ mice (764) was associated with decreased expression of SR-A and TNF- $\alpha$, which may have contributed to the reported reduction of atherosclerosis in mice with elevated adiponectin levels $(516,764)$. Thus modulation of scavenger receptor expression and function by various cytokines may greatly affect lipid metabolism in an inflammatory context.

Besides their effects on scavenger receptor expression and lipid transport in macrophages, cytokines modulate the ability of monocytes/macrophages to oxidize LDL. IFN- $\gamma$ inhibits the macrophage-induced oxidation of LDL (129, 206), whereas TNF- $\alpha$ (458), IL-4, and IL-13 (204) enhance the ability of cell-mediated oxidation.

\section{Effect of SMC migration/proliferation}

In addition to classic survival and growth factors such as PDGF, cytokines have been shown to differentially affect SMC migration and proliferation. One of the first cytokines to be studied in this context was IL-1. IL-1 is a potent mitogen for human SMC, even though it also induces in the short term the production of endogenous prostanoids with growth-inhibitory properties, suggesting both positive and negative, temporally distinct, effects on SMC proliferation (400). An important debate occurred regarding the role of IFN- $\gamma$ on SMC proliferation. Initial in vitro and in vivo studies using models of mechanical injury in T-cell competent or deficient animals reported a cytostatic effect of this T cell-derived cytokine on SMC (266, 267, 723). In contrast, others have reported a promoting effect of IFN- $\gamma$ on SMC in culture (776) and a lack of effect of T-cell deficiency on injury-induced neointima formation using athymic rmu/rmu rats (202). A carefully conducted study has shown that IFN- $\gamma$ indeed elicits SMC proliferation and intimal hyperplasia in a model of transplantation of pig or human arteries into the aorta of immunodeficient mice (670). IFN- $\gamma$ was not found to be 
directly mitogenic, but potentiated the proliferative effect of PDGF-BB under low-serum conditions and upregulated PDGF- $\beta$ receptors (670).

\section{Modulation of extracellular matrix remodeling}

Pro- and anti-inflammatory cytokines produced during atherosclerosis significantly affect the expression of MMPs and their inhibitors TIMPs, acting synergistically with other cytokines, growth factors, or oxidized lipids to induce substantial remodeling of many components of the extracellular matrix (see Ref. 500 for review). The production of type I and III collagen by SMC is slightly increased by IL- 1 and TNF- $\alpha$, whereas TGF- $\beta$ is a potent inducer of collagen synthesis. In contrast, IFN- $\gamma$ inhibits collagen synthesis (17). The proinflammatory IL-1 and TNF- $\alpha$ induce a broad range of MMPs in vascular cells, including MMPs-1, $-3,-8$, and -9 . Cell contact with Tlymphocyte membranes and addition of recombinant CD40 ligand further upregulates a broad spectrum of MMPs in EC and SMC. Proinflammatory cytokines, including IL-1 and TNF- $\alpha$, upregulate macrophage metalloelastase MMP-12, which favors monocyte migration, and MT1-MMP (MMP-14) and MT3-MMP (MMP-16) expression, which could lead to significant basement membrane turnover through activation of constitutive vascular MMP-2. Of note, the Th2-type cytokine IL-4 induces the elastolytic MMP-12 (632). MMP-9 expression in macrophages can be further upregulated by IL- 18 and TNF- $\alpha$. As in vascular cells, CD40 ligation further upregulates MMP expression in macrophages. In contrast, anti-inflammatory cytokines inhibit MMP expression. IL-10 and TGF- $\beta$, the most relevant anti-inflammatory cytokines in atherosclerosis, inhibit an array of MMPs, including MMP-9 and MMP-12. The activity of MMPs is negatively regulated by endogenous TIMPs, including TIMP-1, -2 , and -3 constitutively expressed by SMC (reviewed in Ref. 500). Even though TIMP-1 is upregulated in response to CD40 ligation, TIMP-1 and -2 are unaffected by IL-1 or TNF- $\alpha$ (220, 425). In addition, TIMPs may be upregulated by IL-10 and TGF- $\beta$. Therefore, in plaque areas in which proinflammatory cytokine expression prevails over that of IL-10 and TGF- $\beta$, an imbalance between matrix degradation and synthesis might compromise fibrous cap structure and precipitate its rupture.

\section{Mobilization of vascular progenitor cells}

Since the initial isolation and characterization of putative progenitor endothelial cells (28), a number of growth factors and cytokines have been shown to affect their mobilization, homing to injured tissues, proliferation, and function (389). Cytokines play a critical role in stem cell mobilization (reviewed in Refs. 370, 686). One of the most important and clinically relevant molecules for mobilization of CD34+ T cells is G-CSF. It induces proteinase production by leukocytes, allowing disengagement of stem cells from the stromal bone marrow. Moreover, SDF-1 is released into the circulation leading to attraction and exit of CXCR4+ cells from the bone marrow (272). VEGF, SDF-1, and placenta growth factor (PIGF)-induced stem cell mobilization is dependent on MMP-9 (278), which is required for the cleavage of membrane-bound Kit ligand (278). Lack of endothelial nitric oxide synthase (eNOS) in the stromal bone marrow microenvironment leads to defective mobilization. The phenotype of eNOS-deficient mice recapitulated that of MMP9-deficient mice and was rescued by exogenous administration of soluble Kit ligand, which bypasses the requirement for MMP-9-mediated cleavage of mKit (5).

\section{Regulation of neovessel formation/promotion of intraplaque neovascularization}

EC, SMC, as well as inflammatory cells (monocytes/ macrophages and $\mathrm{T}$ lymphocytes) fully participate in the angiogenic process by expressing or inducing the production of cytokines, chemokines, and adhesion molecules that may affect endothelial cell survival, proliferation, migration, and activation. As in atherosclerosis, positive and negative regulators of the inflammatory response greatly affect neovascularization. It appears that (unfortunately?) most proinflammatory and proatherogenic mediators enhance neovessel formation, and vice versa, particularly in a postischemic setting. On the other hand, most anti-inflammatory and antiatherogenic mediators inhibit the neovascularization process. This tradeoff has been referred to as the Janus phenomenon (192) and may have important clinical implications given the risk of compromising postischemic tissue repair while inducing plaque stabilization (by inhibiting the inflammatory/angiogenic mediators), or the risk of favoring plaque progression/destabilization while promoting tissue functional recover after ischemia (by promoting proangiogenic but also proatherogenic pathways). This could be the case for the growth factors VEGF, PlGF, FGF-2, the cytokines IL-1 $\beta$ and TNF- $\alpha$, the chemokines CCL2 and CXCL8, or even leptin, which all have proinflammatory, proatherogenic, and proangiogenic properties $(63,69,192,423)$. On the other hand, IL-10, CXCL9 (Mig), CXCL10 (IP-10), or adiponectin are anti-inflammatory, antiatherogenic, and antiangiogenic mediators. However, CXCL9 and CXCL10 may also recruit high numbers of lymphocytes to the ischemic tissue, which may promote neovascularization. Interestingly, exceptions to the Janus phenomenon have been reported. eNOS is a potent proangiogenic mediator (5), previously shown to limit atherosclerosis development (346). However, the promotion of superoxide production rather than NO by eNOS under certain pathological conditions may exaggerate atherosclerosis (357). In 
our view, an important exception to the fact that proinflammatory cytokines are also proangiogenic is the IL-18 (and potentially IL-12) pathway. As discussed below, IL-18 is a major proinflammatory/proatherogenic cytokine. Inhibition of endogenous IL-18 significantly reduced atherosclerosis in mice (436). Interestingly, the same therapeutic strategy resulted in the stimulation of postischemic neovascularization, identifying an important target for modulation of atherogenesis while promoting postischemic tissue repair.

Pathological examination of coronary lesions made by the group of Renu Virmani (352) revealed that intraplaque hemorrhage is an important process in the evolution of the plaques from a stable phenotype to high-risk unstable lesions. The source of red blood cells within the plaques is believed to be leaky immature microvessels that are present around and within the plaque (352). Areas of intraplaque neovascularization are infiltrated with $\mathrm{T}$ lymphocytes (705), suggesting a major role for T-cellderived cytokines in plaque angiogenesis.

\section{Induction of apoptosis}

Apoptotic cell death occurs during the development and progression of the atherosclerotic plaque. All cell types are involved, with a high predominance of apoptotic macrophages in the lipid core. Macrophage apoptosis may contribute to enlargement of the lipid core, whereas plaque SMC apoptosis may induce a thinning in the fibrous cap, favoring its rupture $(348,405,443)$. The distribution of apoptosis is heterogeneous within the plaque, being more frequent in regions rich in inflammatory cells and proinflammatory cytokines and much less abundant in regions characterized by a significant production of anti-inflammatory cytokines (441). A number of proinflammatory cytokines have been shown to induce SMC and macrophage apoptosis in culture, particularly the association of IL- 1 , TNF- $\alpha$, and IFN- $\gamma$ and promotion of Fas-FasL killing $(222,223)$. Also, macrophages themselves induce SMC apoptosis through direct and autocrine mechanisms involving TNF- $\alpha$, inducible NO, and Fas/FasL interactions $(79,80)$. Importantly, although physiological programmed apoptosis is essentially a noninflammatory process, apoptosis induced during pathological conditions might per se contribute to disease progression through its inflammatory potential. This could be the case of apoptosis induced through caspase-1 activation, which also releases the active forms of the proinflammatory cytokines IL- $1 \beta$ and IL-18 (339, 667). Other proinflammatory caspases include caspase- 4 , -5 , and -13 . Caspase- 1 and caspase- 5 associate with PYCARD/ASC and NALP1 and together form the inflammasome, which results in the activation and processing of IL- $1 \beta$ and IL-18.

\section{Modulation of procoagulant activity and fibrinolysis}

The antithrombotic properties of EC are deeply altered by IL-1 and by TNF- $\alpha$ and endotoxin (193, 216, 451). They can increase the tissue procoagulant activity and suppress the anticoagulant activity mediated by the thrombomodulin-protein $\mathrm{C}$ system, by decreasing gene transcription of thrombomodulin and protein $\mathrm{C}$ receptor. Downregulation of protein $\mathrm{C}$ pathway limits protein $\mathrm{C}$ activation and thus promotes thrombus formation. In addition, vascular heparin-like molecules are reduced by inflammatory cytokines (344). Downregulation of anticoagulant mediators may in turn affect inflammation. Thrombomodulin has direct anti-inflammatory activities on the endothelium, inhibiting MAPK and NF- $\kappa$ B pathways (138), and activated protein $C$ has been shown to inhibit $\mathrm{NF}-\kappa \mathrm{B}$ in monocytes. On the other hand, proinflammatory cytokines modify the fibrinolytic properties of EC; they decrease the production of tissue plasminogen activator (tPA), and they increase the production of the inhibitor of tissue plasminogen activator (PAI-1). PAI-1 levels rise substantially in response to an inflammatory challenge (597). The increased PAI-1 levels severely impair the ability to remove the thrombus. In addition, inflammatory mediators like IL-6 increase platelet production and thrombogenicity (99).

\section{Regulation of immune response}

For dendritic cell maturation and Th1/Th2/Treg development/maintenance, see section vI.

\section{CYTOKINE AND CYTOKINE RECEPTOR- ASSOCIATED MODULATION OF PLAQUE DEVELOPMENT AND STABILITY}

The generation of a number of mouse models of experimental atherosclerosis using apoE ${ }^{-/-}$or $\mathrm{LDLr}^{-/-}$ mice crossed with mice deficient in genes encoding cytokines or cytokine receptors has been instrumental in our understanding of their role in atherosclerosis development and progression (Table 7) (see review in Refs. 521, 709).

\section{A. Proinflammatory Cytokines}

\section{1. $T N F-\alpha$}

Experimental studies using TNF-deficient apoE $\mathrm{E}^{-/-}$ mice showed that atherosclerotic lesion size in the aortic sinus of $\mathrm{TNF}-\alpha^{-/-} \mathrm{apoE}^{-/-}$mice is significantly smaller than that of apoE $\mathrm{E}^{-/-}$mice, associated with decreased expression of ICAM-1, VCAM-1, and MCP-1 (515). Surprisingly, antiatherogenic property of TNF- $\alpha$ 
TABLE 7. Effect of cytokine deletion on atherosclerosis in murine models of atherosclerosis

\begin{tabular}{|c|c|c|c|c|c|c|c|c|c|}
\hline Cytokine & $\operatorname{Age}(w)$ & Sex & Chol. & Lesion Size & Mac & $\mathrm{T}$ Cells & SMC & Collagen & Reference Nos. \\
\hline \multicolumn{10}{|l|}{ IL- $1 \beta$} \\
\hline IL-1 $1 \beta^{-/-} \operatorname{apoE}^{-/-}$ & 12 & $?$ & $\mathrm{NC}$ & $-33 \%$ & ND & ND & ND & ND & 342 \\
\hline IL-1 $\beta^{-/-}$apoE $^{-/-}$ & 24 & $?$ & $\mathrm{NC}$ & $-32 \%$ & ND & ND & ND & ND & 342 \\
\hline \multicolumn{10}{|l|}{ IL-1ra } \\
\hline $\mathrm{IL}_{-1 \mathrm{ra}^{+/-}}$apoE $^{-/-}$ & 16 & $\mathrm{M}$ & $\mathrm{NC}$ & $+30 \%$ & $\mathrm{NC}$ & ND & $\mathrm{NC}$ & ND & 307 \\
\hline IL-1ra ${ }^{+/-}$apoE $^{-/-}$ & 32 & M & $\mathrm{NC}$ & $\mathrm{NC}$ & $+90 \%$ & ND & $-15 \%$ & ND & 307 \\
\hline TgIL-1raxLDLr ${ }^{-/-} \mathrm{HFD}$ & $14(4+10)$ & $?$ & $+40 \%$ & $\mathrm{NC}$ & ND & ND & ND & ND & 169 \\
\hline IL-1ra ${ }^{+/-}($cholate $)$ & $16(4+12)$ & $?$ & $-25 \%$ & $+50 \%$ & ND & ND & ND & ND & 169 \\
\hline IL-1ra ${ }^{-/-}$(cholate) & $16(4+12)$ & $?$ & $-60 \%$ & $+180 \%$ & ND & ND & ND & ND & 169 \\
\hline ReIL-1ra $>$ apoE $E^{-1-}$ & 12 & $\mathrm{M} / \mathrm{F}$ & $\mathrm{NC}$ & $-74 \% /-56 \%$ & ND & ND & ND & ND & 189 \\
\hline TgIL-1raxapoE ${ }^{-/} \mathrm{HFD}$ & $20(10+10)$ & $\mathrm{M}$ & $+70 \%$ & $-53 \%$ & ND & ND & ND & ND & 462 \\
\hline TgicIL-1raxapoE ${ }^{-1-}$ HFD & $20(10+10)$ & M & $+90 \%$ & $-67 \%$ & ND & ND & ND & ND & 462 \\
\hline \multicolumn{10}{|l|}{$\mathrm{IL}-2$} \\
\hline RecIL-2 $>$ apoE $^{-/-}$ & $?$ & $?$ & $?$ & $+24 \%$ & $?$ & ? & $?$ & ? & 685 \\
\hline Anti-IL-2>apoE & $?$ & $?$ & $?$ & $-43 \%$ & $?$ & $?$ & $?$ & $?$ & 685 \\
\hline \multicolumn{10}{|l|}{ IL-4 } \\
\hline IL- $4^{-/-}($cholate $)$ & $20(5+15)$ & $\mathrm{F}$ & $\mathrm{NC}$ & $\mathrm{NC}$ & $\mathrm{NC}$ & $\mathrm{NC}$ & ND & ND & 227 \\
\hline $\mathrm{IL}-4^{-/-}$apoE $^{-/-}$ & 30 & $\mathrm{M} / \mathrm{F}$ & $\mathrm{NC}$ & $-34 \% /-23 \%$ & $\mathrm{NC}$ & $\mathrm{NC}$ & ND & ND & 156 \\
\hline IL- $4^{-/-}$apoE $^{-/-}$ & 45 & $\mathrm{M} / \mathrm{F}$ & $\mathrm{NC}$ & $\mathrm{NC} / \mathrm{NC}$ & $\mathrm{NC}$ & $\mathrm{NC}$ & ND & ND & 156 \\
\hline BMT IL- $4^{-1-}>\mathrm{LDLr}^{-1-}$ & $46(42+4)$ & $\mathrm{F}$ & $\mathrm{NC}$ & $\begin{array}{c}\text { NC(AS) } \\
-67 \% \text { (aorta) }\end{array}$ & $\mathrm{NC}$ & $\mathrm{NC}$ & ND & ND & 340 \\
\hline \multicolumn{10}{|l|}{ IL-5 } \\
\hline BMT IL-5 ${ }^{-/-}>\mathrm{LDLr}^{-/-}$ & $28(12+16)$ & $\mathrm{F}$ & $\mathrm{NC}$ & $\begin{array}{c}+18 \%(\mathrm{AS}) \\
+54 \%(\text { aorta })\end{array}$ & ND & ND & ND & ND & 53 \\
\hline \multicolumn{10}{|c|}{$10+/ 0(a) i d a)$} \\
\hline RecIL-6 >C57BL6 & $24(3+21)$ & $\mathrm{M}$ & $\mathrm{NC}$ & $+400 \%$ & ND & ND & ND & ND & 294 \\
\hline RecIL-6 $>$ apoE $^{-1-}$ & $9(3+6)$ & M & $\mathrm{NC}$ & $+89 \%$ & ND & ND & ND & ND & 294 \\
\hline RecIL-6 $>$ apoE $^{-/-}$ & 24 & $\mathrm{M}$ & $\mathrm{NC}$ & $+138 \%$ & ND & ND & ND & ND & 294 \\
\hline IL- $6^{-1-}$ apoE ${ }^{-1-}$ & 53 & $\mathrm{~F}$ & $\mathrm{NC}$ & $+85 \%$ & ND & ND & ND & ND & 187 \\
\hline IL-6 ${ }^{-1-}$ apoE $^{-/-}$ & $53 \pm 4$ & M & $+64 \%$ & $+87 \%($ aorta $)$ & $-67 \%$ & $-50 \%$ & ND & $-20 \%$ & 598 \\
\hline \multicolumn{10}{|l|}{ IL-9 } \\
\hline RecIL-9 > LDLr $^{-/-}$ & $?$ & $?$ & $\mathrm{NC}$ & $-65 \%$ & ND & ND & ND & ND & 359 \\
\hline \multicolumn{10}{|l|}{ IL-10 } \\
\hline IL-10 ${ }^{-/-}(\mathrm{C} 57 \mathrm{BL} 6)$ (cholate) & $24(8+16)$ & $\mathrm{F}$ & $\mathrm{NC}$ & $+200 \%(\mathrm{SPF})$ & ND & ND & ND & ND & 434 \\
\hline & & & & $+3000 \%(\mathrm{CONV})$ & $\mathrm{NC}$ & $+350 \%$ & ND & $-75 \%$ & 434 \\
\hline IL-10 ${ }^{-/-}$(C57BL6) (cholate) & 15 & $?$ & $\mathrm{NC}$ & $+150 \%(\mathrm{SPF})$ & ND & ND & ND & ND & 542 \\
\hline IL-10TgC57BL6 (cholate) & 15 & $?$ & $\mathrm{NC}$ & $-60 \%$ & ND & ND & ND & ND & 542 \\
\hline $\mathrm{IL}-10^{-\rho-} \mathrm{apoE}^{-1-}$ & 16 & $\mathrm{M} / \mathrm{F}$ & $\mathrm{NC}$ & $\mathrm{NC} /+180 \%$ & $\mathrm{NC}$ & $\mathrm{NC}$ & $\mathrm{NC}$ & ND & 104 \\
\hline $\mathrm{IL}-10^{-/-} \mathrm{apoE}^{-/-}$ & 48 & $\mathrm{M} / \mathrm{F}$ & $-36 \%$ & $\mathrm{NC} / \mathrm{NC}$ & $\mathrm{NC}$ & $\mathrm{NC}$ & $\mathrm{NC}$ & ND & 104 \\
\hline BMT IL10Tg $>\mathrm{LDLr}^{-1-}$ & $30(10+20)$ & M & $\mathrm{NC}$ & $-47 \%$ & $+50 \%$ & No T cell & ND & $+30 \%$ & 541 \\
\hline 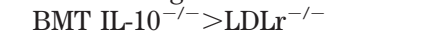 & $32(18+14)$ & $\mathrm{F}$ & $\mathrm{NC}$ & $+35 \%$ & $+61 \%$ & $+116 \%$ & $\mathrm{NC}$ & $-49 \%$ & 548 \\
\hline AdIL-10syst $>\mathrm{LDLr}^{-/-}$ & $20(12+8)$ & $\mathrm{F}$ & $-40 \%$ & $-56 \%$ & - & ND & ND & ND & 708 \\
\hline AdIL-10local $>\mathrm{LDLr}^{-/-}$ & $20(12+8)$ & $\mathrm{F}$ & $\mathrm{NC}$ & $-50 \%$ & - & ND & ND & ND & 708 \\
\hline AdIL-10 $>$ apoE $^{-1-}$ & 13 & M & $\mathrm{NC}$ & $-61 \%$ & $\mathrm{NC}$ & $+89 \%$ & No SMC & $\mathrm{NC}$ & 488 \\
\hline \multicolumn{10}{|l|}{ IL-12 } \\
\hline IL-12 $2^{-/-}$apoE $^{-/-}$ & 30 & $\mathrm{M} / \mathrm{F}$ & $\mathrm{NC}$ & $-48 \% /-59 \%$ & $-40 \%$ & $\mathrm{NC}$ & ND & ND & 156 \\
\hline IL-12 $2^{-/-}$apoE $^{-/-}$ & 45 & $\mathrm{M} / \mathrm{F}$ & $\mathrm{NC}$ & $\mathrm{NC} / \mathrm{NC}$ & $\mathrm{NC}$ & $\mathrm{NC}$ & ND & ND & 156 \\
\hline RecIL-12>apoE ${ }^{-/-}$ & 21 & M & $\mathrm{NC}$ & $+100 \%$ & ND & + & ND & ND & 377 \\
\hline Apobec $^{-/-} \mathrm{LDLr}^{-/-} 12 / 15 \mathrm{Lox}^{-/-}$ & 15 & $\mathrm{M} / \mathrm{F}$ & $\mathrm{NC}$ & $-39 \% / \mathrm{NC}$ & ND & ND & ND & ND & 786 \\
\hline (IL-12 deficient) & 34 & $\mathrm{M} / \mathrm{F}$ & $\mathrm{NC}$ & $-43 \% /-48 \%$ & ND & ND & ND & ND & 786 \\
\hline \multicolumn{10}{|l|}{ IL-18 } \\
\hline IL-18 $8^{-/-}$xapoE $^{-/-}$ & 24 & M & $+50 \%$ & $-35 \%$ & - & $-38 \%$ & $+160 \%$ & ND & 188 \\
\hline RecIL18bp $>$ apoE $^{-/-}$ & 23 & M & $\mathrm{NC}$ & $-25 \%$ & $-50 \%$ & $-67 \%$ & $+100 \%$ & $+85 \%$ & 436 \\
\hline RecIL-18>apoE ${ }^{-/-}$ & 16 & $\mathrm{M}$ & $\mathrm{NC}$ & $+120 \%$ & + & $+350 \%$ & ND & ND & 735 \\
\hline RecIL-18 $>\mathrm{apoE}^{-/-} / \mathrm{IFN}^{-1-}$ & 16 & M & $\mathrm{NC}$ & NC & ND & $\mathrm{NC}$ & ND & ND & 735 \\
\hline AdIL-18 $>$ apoE $^{-/-}$(carotid & & & & & & & & & \\
\hline collar model) & 18 & $\mathrm{~F}$ & $\mathrm{NC}$ & $\mathrm{NC}$ & $-23 \%$ & ND & $\mathrm{NC}$ & $-44 \%$ & 167 \\
\hline RecIL-18>apoE ${ }^{-/-} /$SCID & 14 & $\mathrm{~F}$ & $\mathrm{NC}$ & $+187 \%$ & + & ND & ND & ND & 672 \\
\hline \multicolumn{10}{|l|}{ TNF- $\alpha$} \\
\hline TNF- $\alpha^{-1-}$ (cholate) & $22(6+16)$ & $\mathrm{F}$ & $+46 \%$ & $\mathrm{NC}$ & ND & ND & ND & ND & 614 \\
\hline TNFRp55 ${ }^{-\prime-}$ (cholate) & $24(6+18)$ & $\mathrm{F}$ & $\mathrm{NC}$ & $+130 \%$ & ND & ND & ND & ND & 613,614 \\
\hline TNFRp75 ${ }^{-1-}$ (cholate) & $24(6+18)$ & $\mathrm{F}$ & $\mathrm{NC}$ & $\mathrm{NC}$ & ND & ND & ND & ND & 614 \\
\hline TNFR p $55^{-1-} / \mathrm{p} 75^{-1-}$ (cholate) & $24(6+18)$ & $\mathrm{F}$ & $\mathrm{NC}$ & $+130 \%$ & ND & ND & ND & ND & 614 \\
\hline TNFbp $>$ apoE ${ }^{-/-}$HFD & $13(8+5)$ & $\mathrm{M} / \mathrm{F}$ & $\mathrm{NC} / \mathrm{NC}$ & $\mathrm{NC} /-30 \%$ & $\mathrm{NC}$ & ND & $\mathrm{NC}$ & ND & 189 \\
\hline TmTNF $\times$ C5BL/6 (cholate) & $29(9+20)$ & M & $\mathrm{NC}$ & $-44 \%$ & $-81 \%$ & ND & + & ND & 107 \\
\hline TNF- $\alpha^{-1-}$ diet & $29(9+20)$ & M & $\mathrm{NC}$ & $-94 \%$ & - & ND & ND & ND & 107 \\
\hline TNF- $\alpha^{-1-}$ apoE $^{-1-}$ HFD & $14(4+10)$ & $?$ & $+40 \%(\mathrm{NS})$ & $-50 \%$ (aorta) & $\mathrm{NC}$ & $-50 \%(\mathrm{NS})$ & ND & ND & 83 \\
\hline TNF- $\alpha^{-1-}$ apoE $^{-1-}$ HFD & $44(4+40)$ & $?$ & $\mathrm{NC}$ & $-60 \%$ (aorta) & $\mathrm{NC}$ & $-50 \%(\mathrm{NS})$ & ND & ND & 83 \\
\hline
\end{tabular}


TABLE 7-Continued

\begin{tabular}{|c|c|c|c|c|c|c|c|c|c|}
\hline Cytokine & Age(w) & Sex & Chol. & Lesion Size & Mac & T Cells & SMC & Collagen & Reference Nos. \\
\hline rec $\mathrm{sTNFRI}>\mathrm{apoE}^{-/-}$ & $32(7+25)$ & M & $+62 \%(\mathrm{NS})$ & $-75 \%$ & $\mathrm{NC}$ & $+240 \%$ & ND & ND & 83 \\
\hline BMT TNF $>$ apoE HFD & $35(10+25)$ & $\mathrm{M}>\mathrm{F}$ & $\mathrm{NC}$ & $-83 \%$ & ND & ND & ND & ND & 83 \\
\hline TNF- $\alpha^{-\prime-}$ apoE $^{-1-}$ & 12 & $\mathrm{M} / \mathrm{F}$ & $\mathrm{NC} / \mathrm{NC}$ & $-20 \% /-32 \%$ & ND & ND & ND & ND & 515 \\
\hline $\mathrm{TNF}^{-/-}$apoE*3Leiden HFD & $28(8+20)$ & $\mathrm{F}$ & $\mathrm{NC}$ & $\mathrm{NC}$ & $\mathrm{NC}$ & $\mathrm{NC}$ & $\mathrm{NC}$ & $\mathrm{NC}$ & 67 \\
\hline \multicolumn{10}{|l|}{ TNF- $\beta$} \\
\hline Lymphotoxin $\alpha^{-/-}$(cholate) & $22(6+16)$ & $\mathrm{F}$ & $-20 \%(\mathrm{NS})$ & $-63 \%$ & ND & ND & ND & ND & 614 \\
\hline \multicolumn{10}{|l|}{ IFN- $\gamma$} \\
\hline IFN- $\gamma \mathrm{R}^{-1-} \mathrm{xapoE}^{-/-} \mathrm{HFD}$ & $17(5+12)$ & $\mathrm{F}$ & + & $-60 \%$ & - & - & ND & + & 260 \\
\hline recIFN- $\gamma>$ apoE $^{-1-} \mathrm{HFD}$ & $23(16+7)$ & M & $\mathrm{NC}$ & $+100 \%$ & + & $+170 \%$ & ND & + & 736 \\
\hline IFN- $\gamma^{-1-} \mathrm{xapoE}^{-1-} \mathrm{HFD}$ & & M & $\mathrm{NC}$ & - & - & & ND & & 735 \\
\hline IFN- $\gamma^{-1-}$ xapoE $^{-1-}$ HFD & & $\mathrm{F}$ & $\mathrm{NC}$ & $\mathrm{NC}$ & $\mathrm{NC}$ & $\mathrm{NC}$ & ND & & 735 \\
\hline $\mathrm{INF} \gamma^{-1-} \mathrm{xLDLr}^{-1-}$ & 8 & $\mathrm{M} / \mathrm{F}$ & $\mathrm{NC}$ & $-75 \%$ & - & - & ND & $-(?)$ & 95 \\
\hline $\mathrm{INF}^{-/-} \mathrm{xLDLr}^{-/-}$ & 20 & $\mathrm{M} / \mathrm{F}$ & $\mathrm{NC}$ & $-43 \%$ & $\mathrm{NC}$ & $\mathrm{NC}$ & $\mathrm{NC}$ & $\mathrm{NC}$ & 95 \\
\hline BMT IFN- $\gamma^{-1-}>\mathrm{LDLr}^{-/-} \mathrm{HFD}$ & $15(9+6)$ & $\mathrm{M} / \mathrm{F}$ & NC & $+80 \%$ & $\mathrm{NC}$ & $\mathrm{NC}$ & $\mathrm{NC}$ & + & 507 \\
\hline BMT IFN- $\gamma^{-1-}>\mathrm{LDLr}^{-/-} \mathrm{HFD}$ & $21(9+12)$ & $\mathrm{M} / \mathrm{F}$ & $\mathrm{NC}$ & $\mathrm{NC}$ & NC & $\mathrm{NC}$ & NC & + & 507 \\
\hline \multicolumn{10}{|l|}{ TGF- $\beta$} \\
\hline TGF- $\beta 1^{+/-}$(cholate) & $20(8+12)$ & $\mathrm{F}$ & $\mathrm{NC}$ & $+500 \%$ & + & ND & - & ND & 245 \\
\hline AbTGF- $\beta>$ apoE $^{-1-}$ HFD & $15(6+9)$ & M & $\mathrm{NC}$ & $+82 \%$ & $+30 \%$ & $\mathrm{NC}$ & $\mathrm{NC}$ & $-54 \%$ & 438 \\
\hline sTGF- $\beta$ RII $>$ apoE ${ }^{-/-}$ & $17(5+12)$ & M & $\mathrm{NC}$ & $\mathrm{NC}$ & $-15 \%$ & $+177 \%$ & $\mathrm{NC}$ & $\mathrm{NC}$ & 421 \\
\hline sTGF- $\beta$ RII $>$ apoE $^{-1-}$ & $29(17+12)$ & $\mathrm{M}$ & $\mathrm{NC}$ & $-37 \%$ & $\mathrm{NC}$ & $+145 \%$ & $\mathrm{NC}$ & $-50 \%$ & 421 \\
\hline \multicolumn{10}{|l|}{ BMT CD2-dnTGF-RII $>\mathrm{LDLr}^{-1}$} \\
\hline$-\mathrm{HFD}$ & $22(12+10)$ & M & $\mathrm{NC}$ & $-29 \%$ & $\mathrm{NC}$ & $+75 \%$ & $-33 \%$ & $-29 \%$ & 236 \\
\hline CD2-dnTGF-RIIxapoE ${ }^{-/-}$ & 12 & $\mathrm{~F}$ & $-26 \%$ & $+300 \%$ & + & + & $\mathrm{NC}$ & - & 572 \\
\hline
\end{tabular}

NC, no change; ND, not determined; SPF, specific-pathogen free; CONV, conventional environment; Tg, transgenic; icILra, intracellular IL-1ra; HFD, high-fat diet: in the age column, first is age of animals at death; in parentheses, age at the start of diet + duration of diet. Cholate indicates HFD containing cholate. BMT, bone marrow transplantation: in the age column, first is age of animals at death; in parentheses, age at the start of diet + duration of diet. AS, aortic sinus; NS, not statistically significant; Mac, macrophage.

has also been reported. It has been shown that TNF$\mathrm{R} 1(\mathrm{p} 55)$-deficient $\mathrm{C} 75 \mathrm{BL} / 6$ mice fed an atherogenic diet developed larger lesions than did wild-type C75BL/6 mice, suugesting that TNF-R1 signaling has protective action against atherosclerosis $(613,614)$. In contrast, the same group found that deficiency in TNF- $\alpha$ or in TNFR2(p75) did not affect lesion development, whereas deficiency in lymphotoxin- $\alpha$ (TNF- $\beta$ ) was associated with smaller lesions $(613,614)$. One possible explanation for a favorable effect of TNF- $\alpha$ on atherosclerosis is that TNF- $\alpha$ downregulates scavenger receptor gene and protein expression in macrophages (291, 692), leading to decreased LDL uptake by macrophages and foam cell formation. However, it is noteworthy that the lack of effect of TNF- $\alpha$ deficiency was observed despite a significant $46 \%$ increase in cholesterol levels in TNF- $\alpha^{-1-}$ mice compared with wild-type animals (614). In these conditions of increased cholesterol levels, an absence of effect of TNF- $\alpha$ on the atherosclerotic process should have resulted in enhanced lesion development. The most likely reason for the discrepancy between previous studies analyzing the role of TNF- $\alpha$ on experimental atherosclerosis in mice is the difference in the underlying mechanism of atherogenesis between apo: $\mathrm{E}^{-1-}$ mice fed a normal chow diet and C57BL/6 mice fed an atherogenic diet containing cholate. Schreyer et al. (613) employed TNFR1 ${ }^{-1-}$ C57BL/6 mice fed an atherogenic diet, whereas Ohta et al. (515) used TNF- $\alpha^{-1-} \mathrm{apoE}^{-/-}$mice fed a normal chow diet. In support of a deleterious effect of TNF- $\alpha$ in atherosclerosis, Canault et al. (107), exploring the role of a noncleavable transmembrane form of TNF- $\alpha$ (tm $\mathrm{TNF}-\alpha$ ) in the development of early atherosclerotic lesions, found that the order of the severity of atherosclerotic lesions in C57BL/6 mice fed a cholate-containing high-fat diet was wild-type mice $>\operatorname{tm}$ TNF- $\alpha$ mice $>$ TNF- $\alpha^{-1-}$ mice.

\section{2. $I L-1$}

The pathogenic role of IL-1 has been investigated in apoE $\mathrm{E}^{-/-}$mice fed a cholesterol-rich diet receiving subcutaneous administration of recombinant human IL-1ra (189), and in $\mathrm{LDLr}^{-/-}$mice (169) or apoE ${ }^{-/-}$mice (462) crossed with transgenic mice expressing high levels of IL-1ra. Overexpression of IL-ra increased total cholesterol levels by $\sim 50 \%$, and in spite of this decreased the size of atherosclerotic lesions by $50-70 \%$. In contrast, IL-1ra knockout C57BL/6J mice fed a cholesterol/cholate diet had a threefold decrease in non-HDL cholesterol and a trend toward increased foam-cell lesion area compared with wild-type littermate controls (169). Taken together, these results clearly indicate that IL-1 contributes to atherosclerosis in mice. Further evidence for an important role of IL- $1 \beta$ in atherosclerosis is provided by experiments showing that IL-1 $\beta$ deficiency in $\operatorname{apoE}^{-/-}$mice hampers lesion development (342). 


\section{3. $I L-2$}

IL-2 has long been recognized as a T-cell growth factor, but deficiency of IL-2 has surprisingly no consequences. Mice lacking either IL-2 (610) or its receptors (IL-2R $\alpha / \mathrm{CD} 25$ and IL-2R $\beta$ ) have normal development of $\mathrm{T}$ cells but show severe lymphoproliferative disease (364). These data have been interpreted to indicate that IL-2 has an essential role in controlling self-tolerance. Indeed, a crucial function of IL-2 is to act as the primary growth factor for Treg cells $(432,623)$. Even though IL-2 is expressed in atherosclerotic plaques, its direct role in atherogenesis has not been studied. Only one study reported that intraperitoneal injections of IL-2 or anti-IL-2 antibody in apo- $\mathrm{E}^{-1-}$ mice fed an atherogenic diet increased or decreased, respectively, lesion size (685). These results would indicate that IL-2 is an atherogenic cytokine in apoE $\mathrm{E}^{-/-}$mice. However, in the absence of information on the effect of IL-2 on lipid profiles and on the composition of atherosclerotic lesions, it is premature to conclude on the definitive role of IL-2 in atherosclerosis, given the function of IL-2 in immune tolerance.

\section{4. $I L-6$}

IL-6 has been shown to enhance fatty lesion development in mice (294). IL-6 treatment of $\mathrm{C} 57 \mathrm{Bl} / 6$ mice at supraphysiological concentrations resulted in an about fivefold increase in fatty streak size, whereas treatment of apoE $\mathrm{E}^{-/-}$mice on low- or high-fat diets resulted in about twofold increases (294), suggesting that IL-6 is a proatherogenic cytokine. However, 1-yr-old IL- $6^{-/-}$apoE $^{-/-}$ mice show enhanced plaque formation $(187,598)$. Serum cholesterol levels were found increased in one study (598), but not in the other (187). Increased atherosclerosis in IL- $6^{-/-}$apoE $\mathrm{E}^{-/-}$mice was associated with reduced collagen content in the plaques, blunted synthesis, and release of IL-10 and diminished recruitment of inflammatory cells into the atherosclerotic plaque (598). At $1 \mathrm{yr}$ of age, mice showed more calcified lesions (187). In younger 16-wk-old IL- $6^{-/-}$apoE $^{-/-}$mice, no significant difference in fatty streaks was detected compared with $\mathrm{IL}^{-6^{+/+}}$ apoE $\mathrm{E}^{-1-}$ mice. Therefore, the role of IL- 6 in atherosclerosis appears ambivalent. Similarly, IL-6 can be viewed as a proinflammatory cytokine but may also be regarded as an anti-inflammatory cytokine as it induces the synthesis of IL-1ra and release of soluble TNFR leading to reduced activity of proinflammatory cytokines $(37,675,753)$. It also inhibits macrophage SR-A (393).

\section{5. $I L-12 / I L-18 / I F N-\gamma$}

Previous studies have shown that IFN- $\gamma$ plays a major role in atherosclerosis. IFN- $\gamma$ receptor deficiency was associated with a reduction in atherosclerotic lesion size in $\operatorname{apoE}^{-/-}$mice (260), and cholesterol diet-induced ath- erosclerosis in $\mathrm{LDLr}^{-/-}$mice was significantly reduced in the absence of IFN- $\gamma$ (95). Moreover, IFN- $\gamma$ administered intraperitoneally promoted atherosclerosis in $\mathrm{apoE}^{-/-}$ mice (736). It has been suggested that IFN- $\gamma$ may affect atherosclerosis in a gender-specific manner, IFN- $\gamma$ being proatherogenic only in males (735). However, two studies show conflicting results showing effects of IFN- $\gamma$ on atherosclerosis in female mice $(95,260)$.

IFN- $\gamma$ is a Th1 cytokine that is produced by $\mathrm{T}$ and NK cells following synergistic activation by IL-12 and IL-18. Interestingly, IL-12 and IL-18 have both been shown to be pro-atherogenic. IL-12 appears to intervene in the atherosclerotic process during the early phase of the disease in $\mathrm{apoE}^{-/-}$mice (156). Thirty-week-old $\mathrm{IL}-12^{-/-} \mathrm{apoE}^{-/-}$ mice showed increased lesions, while 40-wk-old mice had lesions of equivalent size compared with wild-type $\operatorname{apoE}^{-/-}$mice (156). Also, a selective defect of IL-12 synthesis by macrophages due to 12/15-lipoxygenase deficiency reduced plaque formation in Apobec $1^{-/-} \mathrm{LDLr}^{-/-}$ (786), and injection of IL-12 in apoE ${ }^{-/-}$mice promoted lesion development (377). IL-18 administration increased lesion size in $\mathrm{apoE}^{-/-}$mice (735), and overexpression of its endogenous inhibitor IL-18 binding protein (IL-18BP) reduced atherosclerosis with profound changes in plaque composition leading to a more stable plaque phenotype (436). Furthermore, IL-18-deficient apoE ${ }^{-/-}$mice reproduced findings observed in apoE ${ }^{-/-}$mice in which IL-18 signaling was blocked by overexpression of IL-18BP, with smaller and more stable lesions compared with apoE $\mathrm{E}^{-/-}$ mice (188). It has been suggested that the proatherogenic effect of IL-18 is in fact mediated by IFN- $\gamma$ because the promotion of atherosclerosis by exogenous IL-18 administration was ablated in IFN- $\gamma$-deficient $\mathrm{apoE}^{-/-}$mice (735). However, the proatherogenic effect of IL-18 can occur in the absence of $\mathrm{T}$ cells (671). Intraperitoneal injection of IL-18 in SCID/apoE ${ }^{-/-}$mice led to larger lesions and increased circulating IFN- $\gamma$ compared with mice injected with saline solution. NK cells were the most likely source of IFN- $\gamma$ since the possibility that it was produced by macrophages has been recently questioned (600), and the production of IFN- $\gamma$ by SMC activated with a combination of IL-12 and IL-18 appears to be low and occasional (228).

\section{6. $C D 40 / C D 40 L$}

CD40 is widely expressed on several cell types including leukocytes and vascular cells. The CD40 receptor is activated following ligation with $\mathrm{CD} 40 \mathrm{~L}(\mathrm{CD} 154)$. A large variety of immunological and vascular cells express CD40 and/or CD40L (reviewed in Ref. 605). Both CD40 and CD40L are present in human atherosclerotic plaques. Platelets are a major source of CD40L. CD40L is cryptic in resting platelets but rapidly translocates to the platelet surface after stimulation by agonists, including ADP, 
thrombin, or collagen. CD40L, expressed at the platelet membrane, is cleaved and shed from the platelet surface in a time-dependent manner, over a period of minutes to hours, generating a soluble fragment, soluble CD40L (sCD40L). Although it may also be shed from stimulated lymphocytes, it is estimated that $>95 \%$ of circulating CD40L is derived from platelets (19). Interestingly, only membrane CD40L on the surface of platelets (281) but not platelet-derived SCD40L (282) can activate EC and upregulate adhesion molecules, proinflammatory cytokines and chemokines in vitro. This might limit vascular inflammation following the cleavage of CD40L from the surface of activated platelets (282). However, the important role for $\mathrm{CD} 40 / \mathrm{CD} 40 \mathrm{~L}$ interactions in the atherosclerosis has been clearly established in $\mathrm{apoE}^{-/-}$and $\mathrm{LDLr}^{-/-}$mice. Administration of antibody to CD40L, when given early in the development of atherosclerosis, was shown to inhibit atherosclerotic lesion initiation in $\mathrm{LDLr}^{-/-}$mice (426). However, in $\mathrm{CD}_{40 \mathrm{~L}^{-/-}}$apoE $^{-/-}$mice, advanced plaques, but not initial lesions, were markedly reduced and displayed a more stable phenotype, associated with a reduced macrophage and T-lymphocyte content, compared with plaques in wild-type apoE ${ }^{-/-}$mice (421). Recent studies aimed at evaluating the respective role of vascular and leukocyte CD40L in promoting atherosclerosis showed that CD40L deficiency on hemopoietic cells did not affect atherosclerosis, suggesting that CD40L expression on nonhematopoietic cell types could be responsible for the proatherogenic effects of CD40L $(40,644)$.

\section{Osteopontin}

OPN, also known as Eta-1 (early T lymphocyte activation 1), is a pleiotropic cytokine critical for the generation of Th1 immunity (29). It can be categorized as a proinflammatory proatherogenic cytokine. With the use of OPN-deficient apoE ${ }^{-/-}$mice infused with ANG II, it has been shown that OPN promotes the development of atherosclerosis and abdominal aortic aneurysms (89). OPN appears to specifically promote early inflammatory mechanisms associated with macrophage recruitment in atherosclerotic lesions.

\section{8. $M I F$}

MIF plays an important role in both innate and adaptive immunity (100). It is an integral component of the host antimicrobial alarm system and stress response that promotes the proinflammatory functions of immune cells. Recent in vivo studies applying neutralizing MIF antibodies in models of injury-induced carotid manipulation in $\operatorname{apoE}^{-/-}$and LDL-r ${ }^{-/-}$mice have provided evidence for a role of MIF in atherosclerosis and restenosis $(125,603)$. Moreover, deficiency of MIF in $\mathrm{LDLr}^{-1-}$ mice reduced atherosclerosis, associated with reduced SMC prolifera- tion, cysteine protease expression, and elastinolytic and collagenolytic activities. (528).

\section{B. Anti-inflammatory Cytokines}

The concept of a cascade of proinflammatory cytokines followed by production of anti-inflammatory cytokines has contributed to the dissemination of the idea that the inflammatory process can be separated into a first set of events linked to the inflammatory response and a second one later on, linked to the anti-inflammatory response involved in the resolution of inflammation. Because atherosclerosis is a chronic inflammatory disease, we have put forward the idea that endogenous anti-inflammatory cytokines should intervene in the atherosclerotic process to dampen inflammation.

\section{1. $I L-10$}

IL-10 is a pleiotropic cytokine produced by Th2-type T cells, B cells, monocytes, and macrophages that inhibits a broad array of immune parameters including Th1 lymphocyte cytokine production, antigen presentation, and antigen-specific T-cell proliferation (712). IL-10 has also potent anti-inflammatory properties on macrophages (68) and plays an active role in limiting the inflammatory response in the vessel wall (669). The role of endogenous IL-10 has been clearly established in mouse models of atherosclerosis. We and others have shown that IL-10 deficiency in C57BL/6 mice fed an atherogenic cholatecontaining diet promotes early atherosclerotic lesion formation, characterized by increased infiltration of inflammatory cells, particularly activated $\mathrm{T}$ cells, and by increased production of proinflammatory cytokines (434, 542). Similar results have been reported in $\mathrm{IL}-10^{-/-}$ apoE $\mathrm{E}^{-1-}$ mice fed a chow diet (104). Consistent with a protective role of IL-10 in atherosclerosis, systemic or local overexpression of IL-10 by adenoviral gene transfer in a model of collar-induced carotid atherosclerosis in $\mathrm{LDLr}^{-/-}$mice was found highly efficacious in preventing atherosclerosis (708), and overexpression of IL-10 by activated $\mathrm{T}$ lymphocytes reduced atherosclerosis in $\mathrm{LDLr}^{-/-}$mice (541). More recently, using a model of chimeric $\mathrm{LDLr}^{-1-}$ mice in which bone marrow cells were deficient for IL-10, we provided evidence that leukocytederived IL-10 is instrumental in the prevention of atherosclerotic lesion development and in the modulation of cellular and collagen plaque composition, at least in part, through a systemic immune response modulation (548).

\section{2. $I L-4 / I L-13$}

IL-4 is produced by Th2 lymphocytes, eosinophils, basophils, and mast cells. It promotes synthesis of IgE and allergic response. The effects of IL-4 are generally 
considered as anti-inflammatory. However, a growing body of evidence indicates that IL-4 may play a role in atherosclerosis through induction of inflammatory responses, such as upregulation of VCAM-1 $(219,379,416)$ and MCP-1 (378, 573). Consistent with this hypothesis, transplantation of bone marrow stem cells from IL-4deficient mice in $\mathrm{LDLr}^{-/-}$mice decreased atherosclerotic lesion formation in a site-specific manner (340). Similarly, $\mathrm{IL}-4^{-1-}$ apoE $^{-1-}$ mice had reduced atherosclerosis in the aortic arch, but not in the aortic sinus, compared with $\operatorname{apoE}^{-1-}$ mice (156). In contrast, IL-4 deficiency in C57BL/6 mice fed an atherogenic diet did not affect the development of early lesions (225). However, the same group found that fatty streak formation in IL- $4^{-/-}$mice immunized with HSP65 or Mycobacterium tuberculosis was significantly reduced compared with lesions in wildtype C57BL/6 mice (227).

IL-4 and IL-13 share a common predominant receptor signaling chain IL-4R $\alpha$. As a result, it is expected that IL-13 affects atherosclerosis in a similar way as does IL-4. However, no studies are currently available to confirm this hypothesis.

\section{3. $T G F-\beta$}

TGF- $\beta$ is a potent anti-inflammatory, immunosuppressive and pro-fibrotic cytokine, with major effects on the biology of SMC (see review in Ref. 243). TGF- $\beta 1$ deficient mice die in utero or in the perinatal period because of widespread uncontrolled inflammation (360, $636)$. The anti-inflammatory and profibrotic properties of TGF- $\beta$ are highly suggestive of a potential antiatherogenic role for this cytokine. Indeed, Grainger et al. (244) first showed that serum active TGF- $\beta$ is markedly depressed in patients with advanced atherosclerosis (244), and that TGF- $\beta 1$ heterozygous mice fed a cholate-supplemented atherogenic diet displayed increased endothelial activation and macrophage infiltration in the aortic sinus (245). The critical role of TGF- $\beta$ for SMC matrix production and plaque stability in atherosclerosis was thereafter demonstrated by studies using apo: ${ }^{-/-}$mice. We have shown that treatment of apoE $\mathrm{E}^{-/-}$mice with neutralizing antibodies to TGF- $\beta 1$, TGF- $\beta 2$, and TGF- $\beta 3$ accelerates the development of atherosclerosis, with lesions displaying increased inflammatory cells and decreased collagen content (438). TGF- $\beta$ may therefore reduce inflammation but also contributes to matrix production within lesions. In agreement with this hypothesis, treatment of $\mathrm{apoE}^{-/-}$ mice with a soluble TGF- $\beta$-receptor II protein (TGF-RII: Fc) that inhibits TGF- $\beta$ signaling resulted in larger plaques, with an increased frequency of macrophages and $\mathrm{T}$ cells and decreased collagen content in the atherosclerotic lesions (419, 420). Moreover, intraplaque hemorrhages were frequently observed. We and others established later on that specific inhibition of TGF- $\beta$ signaling in $\mathrm{T}$ cells leads to the development of atherosclerotic plaques with a phenotype that may potentially increase plaque vulnerability to rupture, strongly suggesting an important protective role of endogenous T-cell TGF- $\beta$ activity against vulnerability to atherosclerosis $(236,572)$. Bone marrow transplantation from transgenic mice that express a dominant negative TGF- $\beta$ receptor type II under a T-cell-specific promoter into $\mathrm{LDLr}^{-/-}$mice resulted in increased differentiation of spleen-derived T cells toward both Th1 and Th2 phenotypes (236). Moreover, atherosclerotic plaques of these mice showed increased T-cell infiltration and expression of MHC class II, along with a decrease in SMC and collagen content. Consistent with these findings, apoE ${ }^{-/-}$mice with disrupted TGF- $\beta$ signaling in $\mathrm{T}$ cells exhibited markedly larger atherosclerotic lesions, with a $>100$-fold increase in aortic IFN- $\gamma$ expression compared with apoE ${ }^{-1-}$ littermates (572). The important role of T-cell-TGF- $\beta$ signaling in atherosclerosis suggests that regulatory pathways in adaptive immunity are essential in modulation of the development and progression of the disease (see sect. $\mathrm{vIC}$ ).

\section{Chemokines/Chemokine Receptors}

Recruitment of inflammatory cells in the intima is an essential step in the development and progression of atherosclerosis. This process depends on the local production of chemokines where inflammatory cells are attracted and on the expression of chemokine receptors by these cells.

\section{1. $M C P 1 / C C R 2$}

One of the earliest studies to link chemokines with atherosclerosis in vivo showed that mmLDL injected into mice increased production of JE, the mouse homolog of MCP-1 (392). $\mathrm{LDLr}^{-1-}$ and apoE ${ }^{-1-}$ mice have since been used to confirm this link showing increased expression of MCP-1/JE and/or its receptor, CCR2 (560). Other studies, in CCR2-deficient apoE $\mathrm{E}^{-1-}$ mice, showed a reduction in aortic lesion area compared with apoE KO alone, even though lipid levels remained high $(74,161)$. In another model of atherosclerosis, using mice overexpressing apolipoprotein B, deletion of the MCP-1 gene protected against monocyte recruitment (241). MCP-1-deficient $\mathrm{LDLr}^{-/-}$mice also showed reduced macrophage recruitment, suggesting that the role of MCP-1 in atherosclerosis is to attract CCR2-bearing monocytes into the vessel wall (258). These studies therefore indicate a proof of principle for the role of MCP-1 in atherosclerotic lesions.

\section{Fractalkine/CX3CR1}

Fractalkine (CX3CL1) is the unique member of the CX3C subfamily and is expressed in both a soluble and 
membrane-bound form on the surface of inflamed endothelium, which confers to it special properties among the other members of the chemokine family (42). Interestingly, whereas soluble CX3CL1 was reported to recruit lymphocytes and monocytes (42, 529), immobilized forms of CX3CL1 and CX3CL1-expressing human umbilical vein EC have been shown to directly mediate the rapid capture and firm adhesion of leukocytes expressing its receptor CX3CR1 under physiological flow conditions (205, 302). In contrast to cell adhesion mediated by the chemokine $\mathrm{KC}$ (murine CXCL1) through its receptor CXCR2, the CX3CL1-induced firm adhesion is uniquely mediated by direct binding of the chemokine to CX3CR1 and does not require the upregulation and activation of integrins, suggesting that CX3CL1 and CX3CR1 mediate a novel pathway for leukocyte trafficking $(205,302)$. Studies in our group and others suggested that CX3CR1 plays a central role in atherogenesis $(135,385)$. CX3CR 1 deficiency in apoE $^{-/-}$mice, even when restricted to one allele, decreased the development of atherosclerosis with a marked reduction in macrophage accumulation (135).

\section{3. $I L-8 / C X C R 2$}

While high levels of circulating chemokines are associated with poor outcome, they contribute towards deactivating circulating leukocytes and limiting their recruitment towards inflammatory foci. This was first illustrated when Gimbrone et al. (233) showed that IL-8 inhibits neutrophil adhesion to cytokine-activated EC and protects these cells from neutrophil-mediated damage. Neutrophil migration into inflamed compartments is severely impaired in transgenic mice showing high levels of circulating IL-8 (639), and a similar observation was reported for monocyte deactivation to chemoattraction in MCP-1 transgenic mice showing high levels of circulating MCP-1 (587). Injection of IL-8 in rabbits and rats significantly reduced in vivo neutrophil migration towards inflammatory foci (275). Similarly, an altered response of neutrophils to IL-8 was reported in human endotoxemia (18), and an altered chemotaxis to GRO- $\alpha$, GRO- $\beta$, GRO- $\gamma$ and ENA-78, but not to IL-8 of neutrophils was reported in septic subjects and associated with a decreased expression CXCR2 (147). Boisvert et al. (69) showed that the murine homolog of the IL-8 receptor also participates in monocyte/macrophage accumulation in LDL receptor KO mice.

\section{RANTES/CCR5}

RANTES is a chemokine that mediates the trafficking and homing of T lymphocytes, monocytes, basophils, eosinophils, and NK cells via different chemokine receptors (CCR1, -3, -4, and -5). It has been implicated in cardiac inflammatory disorders after organ transplantation or arterial injury $(710,780)$. Its role in atherosclerosis has been suggested by studies showing that treatment of $\mathrm{LDLr}^{-/-}$ mice with $N$-methionylated RANTES (Met-RANTES), a functional CC chemokine antagonist, reduced the extent of atherosclerotic lesions, associated with decreased leukocyte infiltration (695). Of note, levels of CCR5 and CCR2 were significantly decreased in Met-RANTEStreated mice. Therefore, the effects of Met-RANTES could not be solely attributed to the functional inhibition of RANTES activity, but possibly to blockade of CCR2 and CCR5 activities. The latter is however unlikely since CCR5 deficiency in apoE ${ }^{-/-}$mice does not seem to be protective in the early stages of atherosclerosis (365).

\section{5. $M I F$}

It has recently been proposed to group mediators with similar functional patterns, which cannot be structurally classified into the known chemokine subfamilies, as a family termed "chemokine-like" (165). MIF, a pleiotropic inflammatory $\mathrm{T}$ cell and macrophage cytokine, belongs to this subfamily. MIF is involved in immune-mediated diseases, including septic shock and chronic inflammation (reviewed in Ref. 100). A key regulatory role for MIF has been shown in the pathogenesis of immunologically induced kidney disease, suggesting that MIF may be important in immune-mediated disease (368). Consistent with this hypothesis, an upregulation of MIF has been observed in EC, SMC, and macrophages during progression of atherosclerosis in humans (98) and in hypercholesterolemic rabbits (401). Moreover, inhibition of MIF in apoE $^{-/-}$mice by treatment with neutralizing MIF antibodies resulted in a shift in the cellular composition of neointimal plaques toward a more stable phenotype with reduced macrophage and increased SMC content (603), as well reduced circulating levels of inflammatory markers such as fibrinogen, MIF and IL-6 (97).

\section{Hematopoietic Factors/M-CSF}

Macrophages are the predominant cells in atherosclerotic plaques. They are derived from circulating monocytes that adhere to the endothelium and then migrate to the subendothelial space. Macrophages are involved in the formation of the plaque, as evidenced by the decreased atherosclerosis in apoE $\mathrm{E}^{-1-}$ mice deficient in macrophage-stimulating factor (op/op mice), which have decreased blood monocyte differential count (643). It is thus likely that macrophages that enter the intima following primary LDL accumulation to scavenge cholesterol overload, activate EC through cytokine release, which in turn increase endothelial permeability to LDL, triggering a vicious circle.

One important finding is that macrophages in the plaque can multiply in situ in the vessel wall (576). Monocyte-colony stimulating factor (M-CSF), a factor of differ- 
entiation and proliferation of stem cells into monocytes, is locally produced by the endothelial and smooth muscle cells from the human atheromatous plaque (577).

\section{E. Platelet-Derived Factors}

Platelets were first believed to participate in atherogenesis because they can promote the proliferative response of SMC through the release of PDGF after adherence and aggregation at focal sites endothelial denudation (580). This turned out to be an erroneous view of atherogenesis. The role of platelets in the initiation of plaque formation has since been revisited, and a large body of evidence indicates that platelets participate in vascular inflammation and may promote atherosclerotic plaque formation. Circulating activated platelets bind to monocytes to form platelet monocyte aggregates. The interactions of activated platelets with monocytes and atherosclerotic arteries lead to the delivery of the platelet-derived chemokines RANTES (CCL5) and PF4 (CXCL4) to the monocyte surface and endothelium of atherosclerotic arteries (298). In addition, activated platelets injected into $\operatorname{apoE}^{-/-}$mice enhance the development of atherosclerotic lesions compared with mice injected with the supernatant of activated platelets (298). The effect of activated platelets on atherosclerosis is shown to require platelet P-selectin, as the development of atherosclerotic lesions is not affected by the injection of activated platelet lacking P-selectin. P-selectin is also expressed by the activated EC and allows leukocyte adhesion to EC (298). However, the main circulating mass of P-selectin is carried in the platelets and is stored in platelet $\alpha$-granules. When the platelets become activated, P-selectin is expressed at the outer membrane of the platelets, and this allows formation of platelet-leukocyte complex. P-selectin can be shed and released in the plasma from both sources. The role of platelet versus endothelial P-selectin in the development of atherosclerotic plaques has been investigated by using chimeric mice with bone marrow of P-selectin ${ }^{-/-} \operatorname{apoE}^{-/-}$mice or wild-type apoE ${ }^{-/-}$mice transplanted to the recipient from either genotype (96). Endothelial P-selectin is crucial for the promotion of atherosclerotic lesion growth because in its absence only relatively small lesions developed. However, platelet Pselectin also contributed to the lesion development because lesions in wild-type recipients receiving transplants with wild-type platelets were $30 \%$ larger than those receiving P-selectin-deficient platelets and were more frequently calcified (96).

In addition to their effects in early atherosclerosis, as shown above by experimental studies, platelets contribute to the progression of late atherosclerosis. The endothelium over established human plaques often shows focal endothelial loss, with adhesion of a platelet monolayer
$(137,157)$. Incorporation of platelets in plaques after rupture or erosion participate in their episodic expansion.

\section{CYTOKINES AND ADAPTIVE IMMUNITY IN ATHEROSCLEROSIS}

\section{A. Role of T/B Cells in Atherosclerosis}

Adaptive immunity develops when specific molecular epitopes on antigens are recognized by antigen receptors with high specificity and affinity, such as T-cell receptors (TCR) and B-cell receptors (BCR), generated by somatic rearrangements in blast cells. A number of data from humans and mice showed oligoclonal expansion of $\mathrm{T}$ cells within atherosclerotic lesions owing to the preferential expression of a limited number of TCR-variable gene segments $(103,652)$. This suggests that a limited set of candidate antigens mediates the specific T-cell proliferation, the most likely immunodominant antigen being oxLDL. Further studies aimed at the elucidation of the direct role of $\mathrm{T}$ and $\mathrm{B}$ cells in atherosclerosis. There is now ample evidence from experimental studies that the adaptive immune system affects the development of atherosclerosis. The net effect of a deficiency in both $\mathrm{T}$ and $\mathrm{B}$ cells is a $40-80 \%$ reduction in atherosclerotic lesion development, as shown in apoE ${ }^{-/-}$or $\mathrm{LDLr}^{-1-}$ crossed into a recombination activating gene (Rag)-deficient background $(153,154,563,646)$ or crossed with severe combined immunodeficiency (SCID) mice (790). The protective effect is observed when the mice are examined at the early stages of plaque development (646), but also at later stages in the absence of severe hypercholesterolemia (153). The effect may vary according to the site of the lesion, immunodeficiency being protective in the aortic root but not in the thoracic and abdominal aorta (153, 154) or in the brachiocephalic trunk (563). Transfer of $\mathrm{CD} 4+\mathrm{T}$ cells from atherosclerotic apoE ${ }^{-/-}$mice into apoE $^{-/-} \mathrm{x} \mathrm{SCID}^{-/-}$mice enhances atherosclerotic lesion development to a level similar to that of immunocompetent controls (790), indicating a proatherogenic role for $\mathrm{T}$ cells.

Natural killer T (NKT) have also been shown to enhance fatty streak development $(30,430,486,683)$. NKT cells can recognize lipid antigens presented by CD1 molecules. CD1 deficient mice on apoE ${ }^{-/-}$or $\mathrm{LDLr}^{-/-}$background showed significant reduction in early fatty streak development, whereas treatment with $\alpha$-galactosylceramide, a potent and specific NKT cell activator, resulted in an increase in lesion size associated with increased IFN- $\gamma$ and IL- 4 production. The influence of CD1d-restricted NKT cells on lesion size was transient, suggesting that these cells contribute to early fatty streak development but are dispensable for plaque progression.

On the other hand, B cells appear to exert a protective effect. Induction of humoral immunity by immuniza- 
tion of hypercholesterolemic apoE ${ }^{-/-}$mice with oxLDL reduces lesion size in association with the production of high levels of IgM type anti-oxLDL antibodies, probably from B1 cells (16, 212, 224, 525, 789). These cells appear to be stimulated by IL-5 produced by MDA-LDL-specific Th2 cells, generated in response to immunization. The group of Joseph Witztum (627) has shown that the IgM type anti-oxLDL antibodies recognize similar oxidationspecific epitopes on apoptotic cells and are structurally and functionally identical to classic "natural" antiphosphorylcholine antibodies that provide protection against pneumococcal infection (627). Immunization of $\mathrm{LDLr}^{-/-}$ mice with Streptococcus pneumoniae induces high circulating levels of oxLDL-specific T15 IgM, indicating molecular mimicry between epitopes of ox-LDL and S. pneumoniae and leads to a reduction in the extent of atherosclerosis, confirming the protective role of this humoral immune response in murine cholesterol-induced atherosclerosis. Splenectomy-induced increase in atherosclerosis in cholesterol-fed apo: $\mathrm{E}^{-/-}$mice is abrogated by the transfer of purified B cells from the spleens of atherosclerotic apoE $\mathrm{E}^{-/-}$(but not from the spleens of nonatherosclerotic mice), suggesting a protective immunity provided by splenic B cells that were "educated" by prior in vivo exposure to atherosclerotic antigens (102).

Besides the possibility of direct B-cell stimulation by thymus-independent antigens leading to IgM-dominated responses, adaptive immunity requires the presentation of antigen by an antigen presenting cell (dendritic cell and macrophage) to the antigen-specific TCR (signal 1) and typically additional costimulatory signals (signal 2), such as the interaction between CD40L with CD40 or that of CD80/CD86 (B7-1/2) with CD28 (Fig. 4). These costimulatory molecules are present in regions of atherosclerostic plaques of mice and humans $(66,377)$ and are required, at least for initial development of atherosclerotic lesions (94, 421, 426), and in the case of CD40/CD40L, for the perpetuation of plaque inflammation $(418,605)$ in mouse models of atherosclerosis. The role of costimulators in the human atherosclerosis is unknown. The best-established role for costimulators is in the activation of naive $\mathrm{T}$ cells, in which they function to reduce the threshold antigen concentration that can activate them (700). Prolonged exposure to higher doses of antigen, as could be the case in the extended course of atherosclerosis development in humans, may well override the need for costimulation. It is therefore tempting to speculate that while costimulation may be necessary for the initiation of pathogenic immune responses in atherosclerosis, this role may become dispensable during disease progression. We believe that it will be important to examine whether at this stage of disease development, other critical roles of costimulators prevail, such as the contribution to regulatory T-cell function (see below).

\section{B. Cytokines and Pathogenic Immune Response in Atherosclerosis}

\section{Cytokines and DC maturation}

Dendritic cells (DCs) are specialized antigen presenting cells (APCs) that are potent stimulators of both T and $B$ cell-mediated immune responses. DC maturation requires the coordinated action of a number of cytokines and growth factors (reviewed in Ref. 34). Several molecules including CD40, TNFR, and IL-1R have been shown to activate DCs and to trigger their transition from immature antigen-capturing cells to mature APCs. The balance between proinflammatory and anti-inflammatory signals in the local microenvironment, including TNF, IL-1, IL-6, IL-10, and TGF- $\beta$, greatly affect DC maturation, and CCR7 plays a critical role in the homing of DCs to lymph nodes (208, 554). Distinct subsets of DCs elicit distinct T-helper responses (34). IL-12 production by DCs plays a critical role in Th1 differentiation as DCs from IL- $12^{-/-}$mice fail to induce Th1 responses (431). IL-6, IL-13, and OX40ligand (OX40-L), a cell surface molecule belonging to the TNF superfamily (TNFSF) also know as TNFSF4 (719), may play a role in DC-induced Th2 differentiation (34). However, DCs exhibit considerable plasticity. Particularly, the anti-inflammatory cytokines IL-10 and TGF- $\beta$ can convert DCs from cells inducing Th1 to cells inducing Th2 or regulatory $\mathrm{T}$ cells (see below). DCs have been identified in atherosclerotic plaques and may cluster with $\mathrm{T}$ cells within the lesions (60). DCs showed impaired migratory function in hypercholesterolemic mice due to inhibitory signals generated by PAF and oxLDL (410). Whereas these abnormal migratory properties directly affect the atherosclerotic process is still unknown.

\section{Cytokines and Th1 differentiation}

Following the demonstration of a pathogenic role for $\mathrm{T}$ cells in atherosclerosis, several groups have been involved in the characterization of the pathogenic T-cell subsets. Most of the T cells in atherosclerotic plaques are of the CD4 $+\mathrm{T}$ cells expressing $\alpha \beta$-TCR, which interacts with MHC class II molecules. The CD4 $+\mathrm{T}$ cells are the main cytokine-secreting $\mathrm{T}$ cells, although the cytotoxic CD8+ killer cells may also produce cytokines, such as TNF- $\alpha$, lymphotoxin, and IFN- $\gamma$. Characterization of the Th cell type in atherosclerosis was based on the cytokines secreted by the $\mathrm{T}$ cells, which are traditionally divided into Th1 cells, responsible for cell-mediated immunity and secreting IFN- $\gamma$ and IL-2, and Th2 cells, which secrete IL-4, IL-5, IL-10, IL-13, and provide help for antibody production by B cells. Th1 and Th2 cells have a common precursor, and cytokine microenvironment is one of the primary determining factor for Th-cell lineage development. IL-12 and TCR activation are required for the induction of Th1 cells, whereas IL-4 is essential for the induc- 


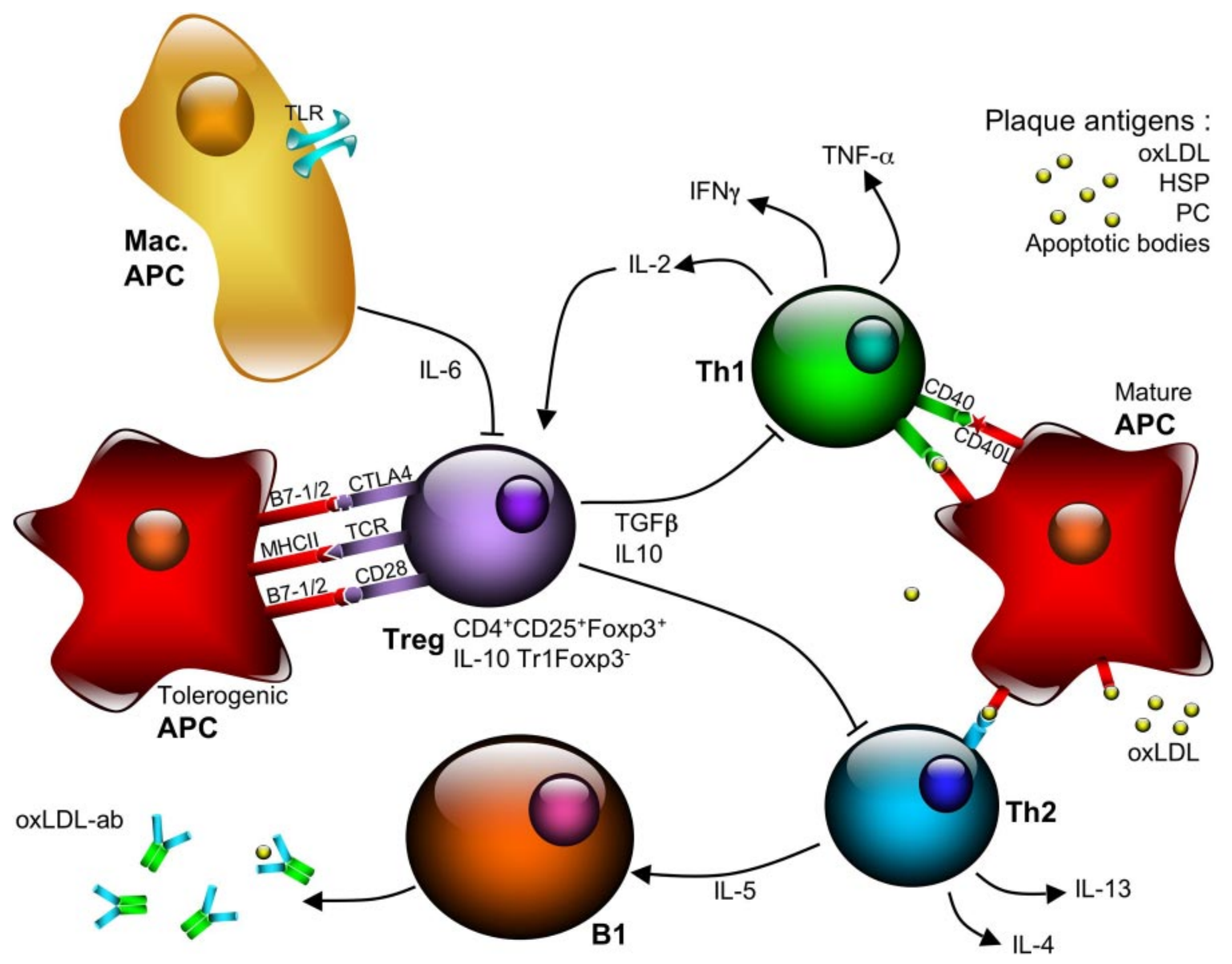

FIG. 4. Development and maintenance of pathogenic and regulatory immunity in atherosclerosis. Candidate antigens [oxidized lipoproteins (oxLDL), heat shock proteins (HSP), phosphorylcholine (PC), apoptotic bodies. . . ] may induce Th1, Th2, or both Th1 and Th2 pathogenic responses. Maturation of the antigen presenting cell (APC) is necessary for T-cell priming. The CD40/CD40L pathway is critical for Th1 differentiation. IL-6 and IL-13 contribute to the induction of Th2 cell type. Both IFN- $\gamma$ (Th1) and IL-4 (Th2) have been shown to promote atherogenesis. Production of IL-5 by Th2 cells is important for protective antibody production by B lymphocytes in response to immunization with oxLDL. Distinct subsets of APCs, called "tolerogenic" cells, induce the differentiation of the regulatory T-cell subset (Treg). The development of CD $4^{+} \mathrm{CD} 25^{+} \mathrm{Foxp} 3^{+}$Treg cells occurs in the thymus and requires TCR and CD28 engagement among other yet unknown factors. Interestingly, production of IL-2 by pathogenic Th1 cells is essential for the survival and maintenance of this Treg subset in the periphery. Other Treg cells of the Tr1 type may not express Foxp3 and are mainly induced in the periphery in response to antigen stimulation. Both types of Treg cells use IL-10 and/or TGF- $\beta$ to suppress the proliferation of pathogenic T cells in vivo. IL-6, produced in response to TLR stimulation, may contribute to the inhibition of Treg function.

tion of antigen-specific Th2 cells. IL-12 originates from macrophages and dendritic cells of the innate immune response, but the initial sources and mechanisms of IL-4 production remain poorly understood. IL-12 activates the transcription factor STAT4 and a unique Th1 transcription factor, T-box expressed in T cells (T-bet), leading to upregulation of IFN- $\gamma$ and downregulation of IL-4 and IL-5 expression in T cells. IL-4 drives Th2 cell differentiation through STAT6, which activates the transcription factor Gata3, leading to upregulation of IL-4 and IL-5 and downregulation of IFN- $\gamma$. Counterregulation between T-bet and Gata3 has been suggested, resulting in inhibition of development of the other T-cell subset (660). Based on these T-cell subset specificities, most CD4 + T cells of atherosclerotic plaques of mice and humans have been shown to be of the Th1 cell type, producing IL- 2 and IFN- $\gamma$ (reviewed in Ref. 751), which is consistent with the high levels of IL-12 expression within the plaques. Subsequent studies have clearly shown a critical pathogenic role for the Th1 response in atherosclerosis at the cell-type level (transfer of Th1 cells) (790), the cytokine production level (IL-12, IL-18, and IFN- $\gamma$ ) (260, 377, 436, 735, 736), and even at the level of Th1 cell commitment, as shown more recently using the $\mathrm{LDLr}^{-/-} \times \mathrm{T}^{-b e t}{ }^{-/-}$mice (94). These results provide convincing elements to incriminate Th1 responses in the promotion of plaque development.

\section{Cytokines and Th1/Th2 paradigm}

Because of the crucial roles of Th1 and Th2 in the modulation of the immune response in many immunoinflammatory diseases, a model has emerged in which Th2biased responses were proposed to antagonize proatherogenic Th1 effects and thereby confer atheroprotection. 
Data supporting an antiatherogenic effect of Th2 responses are based on several, seemingly convincing findings: 1) IL-10, one of the Th2-related cytokines, is expressed in atherosclerotic plaques $(441,687)$ and inhibits oxLDL-induced production of IL-12 by human monocytes in vitro (687); 2) endogenous IL-10 is protective against atherosclerosis in several experimental models (434, 542, 708); 3) mice producing $\mathrm{T}$ cells that were engineered to overexpress IL-10 under the control of the IL-2 promoter show a reduction in IFN- $\gamma$ production and a switch in IgG production toward a Th2-related IgG1 phenotype, associated with a significant decrease in atherosclerotic lesion formation (541); and 4) mice in which Th2 responses prevail over Th1 may show reduction in early fatty streak formation (295).

However, these data are not as straightforward as they may seem to be, and may be opposed by several other findings. 1) Even though IL-10 is a Th2-related cytokine, it is not specific of Th2 cells and has even been shown to inhibit Th2 responses $(145,257)$. 2) Mice overexpressing IL-10 under the IL-2 promoter, discussed above, have been previously shown to be unable to mount Th2 responses (261). 3) Deficiency in IL-4, the prototypic Th2-related cytokine, has been associated with a decrease in atherosclerotic lesion formation (340), suggesting a proatherogenic role of Th2. 4) Prolonged hypercholesterolemia in animal models of atherosclerosis is associated with a switch of the autoimmune response toward a Th2 cell type, producing IL-4 (795), which contribute to plaque progression, since deficiency in IL-4 at these advanced stages greatly hampers plaque progression (156). 5) With the use of $\mathrm{apoE}^{-1-} \times \mathrm{IL}-12^{-/-}$and $\mathrm{apoE}^{-/-} \times \mathrm{IL}^{-4^{-1-}}$ mice, it has been clearly shown that both Th1 and Th2 play roles throughout the development of atherosclerosis, Th1 being predominant during the initiation of lesion formation with a switch toward a proatherogenic Th2 response in the chronic phase of plaque development (156). Therefore, even though atherosclerosis occurs mostly in a Th1-related pathogenic context, no direct and solid evidence is available suggesting that promotion of Th2 responses would invariably lead to limitation of disease progression. The attractive concept of Th1 and Th2 controlling in a Yin-Yang fashion the development of atherosclerosis may be, at least in some circumstances, overly simplistic. However, this does not exclude a certain level of counterregulation between Th1 and Th2 in atherosclerosis, which may vary with the stage of disease development and the vascular sites. For example, in the study by Davenport et al. (156), while deficiency in IL-4 (and hence Th2 response) was associated with a decrease in lesion size in the advanced stage of lesion development, deficiency in IL-12 was associated with a trend toward larger lesions in certain vascular sites, suggesting a potential regulatory role for Th1 response at this advanced disease stage. Given the available data, we would caution about the extreme hazard associated with the promotion of either a Th1 or a Th2 response to modulate atherosclerosis, especially in humans. Indeed, a careful look at the disease in humans shows that Th1- and Th2-related diseases develop and perpetuate in the same patient. A substantial proportion of patients are affected both by coronary atherosclerosis, a Th1-predominant disease, and atherosclerotic aortic aneurysm, a Th2-predominant process (149, 607, 633). In addition, the development of a Th2related disease (allergic asthma, for example) in a given patient does not protect from the development of a Th1related disease (coronary atherosclerosis, for example). A substantial proportion of patients, especially obese patients, frequently develop both allergic asthma and coronary atherosclerosis. An objective interpretation of the available data suggests that Th1- and/or Th2-mediated responses may contribute to the development and progression of atherosclerosis. Therefore, we believe that rather than focusing on a supposedly Th1/Th2 Yin-Yang in atherosclerosis, we should aim at the identification of the causes of Th1/Th2 dysregulation, which we believe could be better explained by a dysfunction in the regulatory arm of the immune response that controls both Th1 and Th2. Our hypothesis is that in the context of atherosclerosis, an imbalance exists between pathogenic $\mathrm{T}$ cells (Th1 and/or Th2) and so-called "regulatory T cells" in response to "altered" self-antigens, leading to reciprocal and mutual amplification of the innate and adaptive immune responses, responsible for plaque development and progression.

\section{Immunological Tolerance and Regulatory T Cells}

\section{Development and function of natural regulatory T cells}

The adaptive immune system of higher vertebrates allows individual organisms to mount more efficient and specific defensive immune reactions against unanticipated microbial antigens by the random generation, in developing lymphocytes, of a diverse repertoire of clonally distributed antigen receptors capable of recognizing a multitude of antigens. This occurs through a process of somatic cell gene rearrangement mediated by the recombination-activating gene recombinase. However, due to the diversity of antigen recognition afforded by the system, there is a considerable risk of self-antigen recognition by self-reactive receptors, posing a concrete risk of autoimmunity. Most solutions to this threat involve the deletion or functional inactivation of autoreactive lymphocytes (clonal deletion and anergy, respectively) in the primary lymphoid organs or in the periphery. These mechanisms are called "cell-intrinsic," since they do not affect other self-reactive clones. In addition to this "reces- 
sive" suppression, a unique "dominant" self-tolerance mechanism has been identified over the past few years and related to the generation of a population of $\mathrm{T}$ cells with regulatory properties, the so-called "regulatory $\mathrm{T}$ cells," which actively suppress immune activation and maintain immune homeostasis.

Most if not all naturally arising Treg cells are CD4 ${ }^{+}$ single-positive cells and constitutively express the CD25 molecule (IL-2R $\alpha$ ). They are produced in the normal thymus where unique interactions between their TCRs and self-peptide/MHC complexes expressed on the thymic stromal cells are required for their development. CD25 expression on Treg cells is crucial for their generation, survival, and function. Mice deficient in IL-2 or CD25 develop a lymphoproliferative disease with autoimmune manifestations, a syndrome that can be rescued by IL-2 administration in IL-2-deficient mice. Interestingly, the responder non-Treg cells are the main source of IL-2 production and are required for the activation and maintenance (and proliferation) of Treg cells. These in turn suppress the pathogenic $\mathrm{T}$ cells by targeting the transcriptional control of IL-2, leading to inhibition of IL-2 production. Thus IL-2 mediates a feedback control mechanism between pathogenic and Treg cells.

Costimulatory signals mediated by engagement of CD28 by CD80/CD86 (B7) are essential for the development and homeostasis of Treg cells (Fig. 4). Mice deficient in CD28 or B7 molecules lack Treg cells and are at increased risk of autoimmune diabetes (591). In turn, CD28 engagement promotes IL-2 production by nonregulatory conventional $\mathrm{T}$ cells, maintaining a stable pool of Treg (Fig. 4).

Even though IL-2 is a vital cytokine for Treg, recent studies suggest that expression of the forkhead transcription factor Foxp3, irrespective of CD25 expression or MHC restriction, defines the naturally occurring Treg cell lineage (207). The critical role of Foxp3 in the control of autoimmune diseases is reflected by the observations that Foxp3 is the mutated gene in the fatal human autoimmune disorder "immune dysregulation, polyendocrinopathy, enteroathy, X-linked" (IPEX) and in the mouse, scurfy, which develops a similar autoimmune syndrome (45, 741). Foxp3 appears to be crucial for both the development and function of Treg cells and controls genes encoding Treg cell-associated molecules, such as CD25, cytotoxic T-lymphocyte antigen-4 (CTLA-4), and glucocorticoid-inducible tumor necrosis factor receptor (GITR) (334). Transduction of Foxp3 in CD25 $5^{-}$cells led to the acquisition of Treg cell properties and CD25 expression in some of the transduced cells, suggesting a central, but probably not sufficient, role for Foxp3 in the development and programming of Treg cell function. The current understanding is that Foxp3 is required for the development of the Treg cell lineage, whereas production of IL-2 by peripheral T cells expands the Treg cell population. The precise molecular mechanisms behind the induction of Treg cells by Foxp3 and the potential role of Foxp3 in the maintenance of Treg cell function remain to be addressed.

Specific subsets of dendritic cells may be critical to the generation of defined populations of Treg (23). Aberrant expression of T-cell receptor agonists by nonactivated hematopoietic cells produces mostly $\mathrm{CD} 4^{+} \mathrm{CD} 25^{-}$ regulatory $\mathrm{T}$ cells, whereas expression on thymic stroma yields predominantly antigen-specific $\mathrm{CD} 4{ }^{+} \mathrm{CD} 25^{+}$Treg. Interestingly, expression of thymic stromal lymphopoietin (TSLP) in the human thymus induces tolerogenic thymic dendritic cells with high expression of CD80 and CD86, leading to the proliferation and differentiation of $\mathrm{CD} 4{ }^{+} \mathrm{CD} 25^{-}$thymic $\mathrm{T}$ cells into $\mathrm{CD} 4^{+} \mathrm{CD} 25^{+} \mathrm{Foxp}^{+}{ }^{+}$regulatory $\mathrm{T}$ cells (725).

\section{Cytokines and regulatory $T$ cells}

Besides the role of IL-2 in the Treg cell development and maintenance, two immunosuppressive cytokines, TGF- $\beta$ and IL-10, have been shown to mediate, at least in part, Treg function in vivo. In fact, Treg cells appear to use various modes of suppression in vivo. Depending on the microenvironment and the immunopathology to be suppressed, Treg cells may act through cell-cell contactdependent mechanisms, particularly engagement of CTLA-4 on B7 molecules, through production of immunosuppressive mediators or both (707).

Recent studies have shown that at least part of the in vivo regulatory function of natural Treg may be due to the induction of IL-10 production in responder $\mathrm{CD} 4^{+} \mathrm{CD} 25^{-} \mathrm{T}$ cells, which in turn become immunoregulatory cells able to suppress certain forms of immunopathology (32, 174, 655). TGF- $\beta$ mediates, at least in part, the suppressive function of natural Treg in vivo and under certain culture conditions in vitro $(44,120,251)$. This TGF- $\beta$-dependent suppressive activity of $\mathrm{CD} 4^{+} \mathrm{CD} 25^{+}$Treg cells is required to inhibit pathogenic $\mathrm{CD}^{+} \mathrm{T}$ cells in models of autoimmunity or tumor rejection $(120,251)$, as shown in models with defective TGF- $\beta$ receptor II signaling in $\mathrm{CD}^{+} \mathrm{T}$ cells. TGF- $\beta$-dependent $\mathrm{CD} 4^{+} \mathrm{CD} 25^{+}$Treg cells are induced in vivo after treatment by antibodies to CD3 and mediate the restoration of self-tolerance in overt autoimmune diabetes (44), leading to limitation of disease progression (333). Whether TGF- $\beta$-dependent suppressive function is also required for inhibition of $\mathrm{CD}^{+} \mathrm{T}$ celldependent immunity in particular settings remain to be determined.

In addition to their role in mediating the suppressive properties of naturally arising Treg cells, TGF- $\beta$ and IL-10 mediate the development and function of adaptive Treg cells induced in the periphery in response to antigen stimulation. These Treg do not express CD25 and have lower Foxp3 expression compared with natural $\mathrm{CD} 4{ }^{+} \mathrm{CD} 25^{+}$Treg cells (Fig. 4). The IL-10 producing Treg 
are known as $\operatorname{Tr} 1$ cells $(255,512,698)$. These cells can be generated by chronic antigenic stimulation or mucosal administration of antigen $(9,654)$ in vivo or under certain culture conditions in vitro (see below) $(255,512,698)$. $\operatorname{Tr} 1$ cells also produce a significant amount of TGF- $\beta$ in addition to IL-10. Other antigen-induced Treg cells, Th3 cells, arise after oral administration of antigen in vivo and suppress immune pathology in several animal models through the production of high concentrations of TGF- $\beta$ $(123,730)$.

The development of these regulatory cells may be promoted in vitro and in vivo by a specific set of antigens and under particular conditions (254). Particularly, administration of antigens via the nasal route leads to IL-10dependent Treg $(9,654)$, whereas oral administration induces the generation of TGF- $\beta$-dependent Treg (123). In vitro culture of bone marrow cells in the presence of IL-10 induces the differentiation of a distinct subset of dendritic cells $\left(\mathrm{CD} 11 \mathrm{c}^{\text {low }} \mathrm{CD} 45 \mathrm{RB}^{\text {high }}\right.$ ) that display plasmacytoid morphology and an immature-like phenotype, secrete high levels of IL-10 after activation, and induce tolerance through the differentiation of $\mathrm{Tr} 1$ cells in vitro and in vivo (713). TGF- $\beta$ and IL-10 production by apoptotic cells or upon ingestion of these cells by macrophages leads to cell deactivation and inhibition of self-reactive T cells (121, 221, 293, 299, 651). Apoptotic cells may also induce the generation of tolerogenic dendritic cells $(10-12,595,596)$, potentially leading to the development of defined populations of Treg. Engulfment of apoptotic cells by dendritic cells in a proinflammatory microenvironment suppressed the upregulation of the costimulatory molecule CD86 and inhibited IL-12 production, leading to a reduced ability to stimulate $\mathrm{T}$ cells. Opsonization of apoptotic cells by the complement $\mathrm{C} 3$ activation product iC3b induces tolerant dendritic cells that are able to migrate to lymph nodes (696). The ligation of iC3b to complement receptor type 3 (the iC3b receptor) on antigen-presenting cells results in the sequential production of TGF- $\beta$ and IL-10, which is essential for the induction of tolerance (645). Moreover, coengagement of CD3 and the complement regulator CD46 in the presence of IL-2 induces a Tr1-specific cytokine phenotype in human $\mathrm{CD}^{+} \mathrm{T}$ cells (332), and transgenic expression of human CD46 in mice promotes a regulatory T-cell response (448).

\section{Regulatory $T$ cells in atherosclerosis}

The findings by several independent groups that two of the major counterregulatory cytokines in atherosclerosis, IL-10 and TGF- $\beta$, are those required for the immunoregulatory functions of either natural or adaptive antigeninduced Treg cells, led to the hypothesis that adaptive or natural regulatory cells may play an important role in the control of the atherosclerotic process (Fig. 5).
Given that immunization of mice with mHSP65 aggravates the development of atherosclerosis, two studies addressed the role of mucosal tolerance to HSP-65 (expected to induce Treg cells) in the development of experimental atherosclerosis $(269,450)$. Harats et al. (269) showed a reduction in lesion size after oral administration of HSP-65 in $\mathrm{LDLr}^{-/-}$mice immunized with $M$. tuberculosis or fed an atherogenic diet (269), suggesting that tolerance induction toward HSP may be protective against atherosclerosis. Although oral feeding with HSP-65 induced a specific immune suppression, the reduction in atherosclerosis could also be obtained with nonsuppressive doses of oral HSP (269). The mechanisms leading to lesion reduction have not been clearly delineated, but the T-cell cytokine profile was switched toward a Th2 phenotype with high production of IL-4. Maron et al. (450) also showed a reduction in atherosclerosis after nasal or oral feeding of HSP-65 while only nasal feeding resulted in significant changes in the T-cell phenotype. No HSP-specific IL-10 responses were detected in splenocytes, but significant IL-10 production was observed following anti-CD3 stimulation in vitro. IL-10 production was attributed to a switch toward a Th2 phenotype and could not be related to changes in IFN- $\gamma$ production by $\mathrm{T}$ cells (450). Taken together, the results of these two studies suggest that mucosal administration of antigen reduces plaque development. However, the mechanisms behind this effect are not fully understood. Additional mechanistic work is required to understand the potential role of the regulatory immune response in this process.

We have recently used a different approach to address the role of the regulatory immune response in atherosclerosis. As our hypothesis implies that an imbalance exists between the effector (Th1/Th2) and the regulatory arms of the immune response, we suggested that supplementation with Treg cells may lead to the induction of immune suppression and a reduction in pathogenic T-cellmediated responses, ultimately altering plaque development and/or composition. In a first step, we attempted to provide a proof of concept that Tr1 cell therapy could induce immunomodulation in vivo and limit plaque development in a model of humanlike atherosclerosis. Therefore, we generated in vitro, as previously described (255), ovalbumin (OVA)-specific Tr1 cells and administered these cells to apoE ${ }^{-1-}$ mice. These antigen-specific clones of $\operatorname{Tr} 1$ cells have been shown to induce both antigenspecific and nonspecific bystander immune suppression in vitro, and when introduced in vivo (255). We showed that the clone of $\operatorname{Tr} 1$ cells, when transferred into mice with their cognate antigen, induced a significant suppression of Th1 (and Th2)-mediated responses and led to an increase in IL-10 production by stimulated peripheral $\mathrm{T}$ cells (437). Interestingly, the induction of Tr1 responses was associated with a significant reduction in atherosclerotic plaque development and a marked reduction in the 


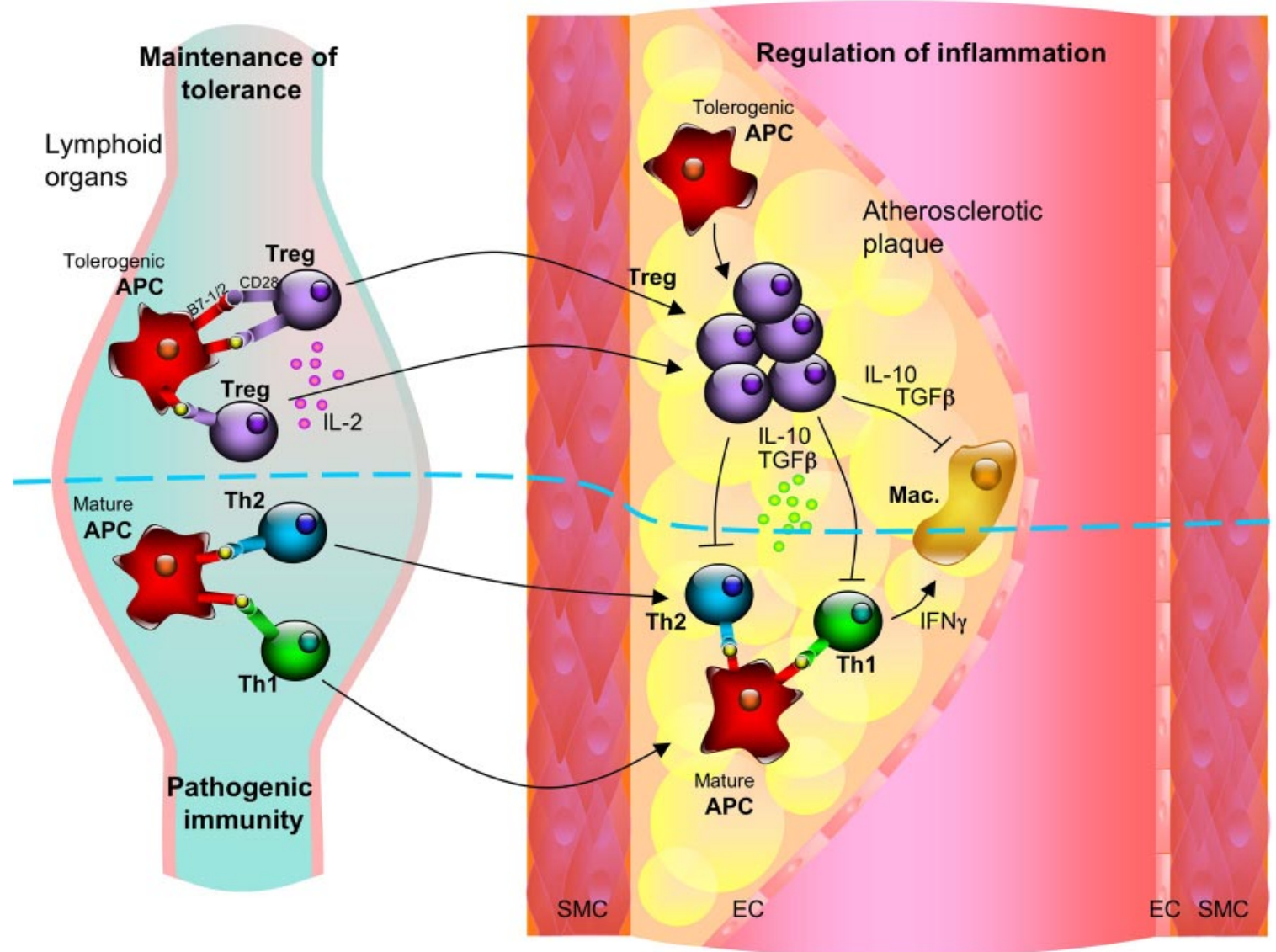

FIG. 5. Local and systemic effects of pathogenic and regulatory cells in atherosclerosis. Lymphoid organs are specialized in antigen presentation and may be the major site of pathogenic or tolerogenic antigen presentation and T-cell priming in atherosclerosis. Antigen presentation may also occur within the atherosclerotic plaque, which is rich in cells with antigen-presenting capacity (macrophages and dendritic cells). Continuous trafficking of immune cells between the inflammed atherosclerotic artery and the lymphoid organs may be necessary to mount an adaptive immune response. CD28 engagement and IL-2 production by pathogenic T cells are required for Treg cell survival and maintenance in the periphery. Treg cells suppress the pathogenic response through IL-10, TGF- $\beta$, and/or cell-cell contact-dependent mechanisms. The precise mechanisms that drive a pathogenic or a regulatory immune response in atherosclerosis are currently unknown.

relative accumulation of inflammatory macrophages and T lymphocytes with a preservation of SMC and collagen contents. These results showed that modulation of the peripheral immune response is achievable by transfer of Tr1 cells with no specificity to a known plaque antigen and leads to limitation of plaque development in apoEdeficient mice. Many issues remain to be addressed, particularly those regarding the precise site(s), mode(s) of action, and molecular mechanisms responsible for the regulatory functions of the transferred $\operatorname{Tr} 1$ cells in this setting, and whether specific and local immune suppression could be achievable by the development and transfer of $\operatorname{Tr} 1$ cells specific for a known plaque antigen.

An important question that has not been addressed in the above-mentioned experiments concerns the role of endogenous natural Treg in the control of atherosclerosis. A number of endogenous self- or altered self-antigens (oxidized epitopes on apoptotic cells or ox-LDL, HSP, for example) may induce the development of Treg cells with atheroprotective properties. We have recently tested the hypothesis that the natural repertoire of Treg cells, which is responsible for the maintenance of immune homeostasis, also limits the development of atherosclerosis. Atherosclerosis in apoE ${ }^{-1-} \mathrm{RAG}-2^{-/-}$mice is exacerbated after transfer of splenocytes with Treg deficiency (from CD28- or B7-deficient mice) compared with the transfer of wild-type splenocytes, a process that is abrogated after the reconstitution of a normal CD4 ${ }^{+} \mathrm{CD} 25^{+}$Treg cell compartment (8). Protection is associated both with enhanced IL-10 production by $\mathrm{CD}^{+}{ }^{+} \mathrm{T}$ cells and TGF- $\beta$-dependent Treg suppressive function, consistent with the critical roles of these immunosuppressive cytokines in atheroprotection. We believe that innate or acquired impairment of natural Treg cell function may promote atherosclerosis.

Defective clearance of apoptotic cells has been described in atherosclerosis $(25,243,615)$. Such a defect may break immunological tolerance and alter both specific and bystander immune suppression, leading to exacerbation of plaque development. Impaired clearance of dying cells appears to play a pathogenic role in the devel- 
opment of autoimmunity $(75,549,617,668)$. Methods aiming at the promotion of endogenous natural Treg cell activity against atherosclerosis-related antigens or methods based on the transfer of antigen-specific Treg cells hold great promise for the control of plaque development and progression through the induction of a regulatory atheroprotective immunity.

Cells other than $\mathrm{CD} 4^{+} \mathrm{T}$ lymphocytes, such as $\mathrm{CD} 8^{+}$, NKT and B cells, may prove to exert potent regulatory properties in atherosclerosis. An IL-10-producing regulatory B-cell subset characterized by CD1d upregulation has been shown to be essential for the dampening of the inflammatory response in a model of chronic intestinal inflammation mediated by a Th2 pathway (474). It is noteworthy in this regard that the protective role of $\mathrm{B}$ cells in atherosclerosis has been shown to occur in a Th2 context $(52,102,268)$.

\section{CYTOKINES AND CARDIOVASCULAR RISK}

Once produced, cytokines are rapidly trapped by neighboring cells via their high-affinity receptors. Accordingly, measuring the levels of circulating cytokines is not necessarily a perfect surrogate end point reflecting the actual activity of the cytokine. Nevertheless, a variety of plasma inflammatory markers have been shown to well predict future cardiovascular risk. They can be useful for risk stratification and also to identify those patients who might benefit from targeted interventional therapy. Of these markers, C-reactive protein (CRP), an acute-phase protein, has been the most extensively studied, and there is now robust evidence from primary prevention cohorts and among patients presenting with ACS that elevated CRP levels predict future cardiovascular events (see review in Ref. 398). The production of CRP occurs almost exclusively in the liver by the hepatocytes as part of the acute phase response upon stimulation by IL-6, and to a lesser degree by TNF- $\alpha$ and IL- $1 \beta$, originating at the site of inflammation. CRP activates the classical complement cascade and mediates phagocytosis. In the 1990s, Berk, Weintraub, and Alexander (46) showed that plasma CRP levels are elevated in patients with "active" CAD compared with those with stable CAD. In 1994, Attilio Maseri and his group (408) established a link between CRP elevation and cardiovascular events in patients with unstable angina (UA). In the late 1990s, several studies linked elevated high-sensitivity CRP (hsCRP) levels with future cardiovascular events in different populations (reviewed in Ref. 56). It is believed that classical cardiovascular risk factors including LDL cholesterol, hypertension, smoking, and diabetes can instigate the vascular release of proinflammatory cytokines and subsequent promotion of lowgrade inflammation. These proinflammatory cytokines increase serum levels of CRP, supporting the concept that
CRP acts as an integrator for many inflammatory stimuli, which in association with plasma LDL-cholesterol levels can predict the cardiovascular risk (569). Of potential clinical interest, the combination of an inflammatory marker (CRP, SAA, SICAM-1, or IL-6) with lipid testing improved upon risk prediction based on lipid testing alone. Thus lipid and inflammatory parameters appear to be assessing different biological pathways that carry separate prognostic value. In support of this hypothesis, the PROVE-IT-TIMI 22 study recently established that the risk of recurrent myocardial infarction (MI) or death from coronary causes among patients with acute myocardial syndromes (ACS) is best predicted by the combination of LDL cholesterol and CRP levels (569).

A number of in vitro studies aimed at investigating the direct inflammatory effects of CRP on vascular cells emphasized the potential importance of CRP as an etiological factor in inflammation and atherosclerosis (see review in Ref. 313). Among other effects, recombinant CRP has been shown to enhance the expression of ICAM-1, VCAM-1, E-selectin, and MCP-1 in EC (533, 534). However, several recent papers have clearly demonstrated that most, if not all, of the in vitro effects of recombinant CRP previously reported in the literature were most likely artifactual and due to the presence of sodium azide $(406,659,689)$ or contamination by bacterial products (539) in the commercial CRP preparation used in the experiments. Morever, in vivo experiments assessing the direct role of CRP on atherosclerosis in CRP transgenic apo: $\mathrm{E}^{-1-}$ mice failed to observe any effect (285, 566 ), or reported a very small effect in male but not female mice (538). There is even some evidence that CRP might be protective against atherosclerosis $(48,616)$ and has a clear anti-inflammatory activity that protects mice from lethality due to LPS challenge (752). The protective effect of CRP appears to be mediated by binding to Fc $\gamma$ RI and FcyRII resulting in enhanced secretion of IL-10 and downregulation of IL-12 (479). It is therefore unlikely that CRP is a mediator of atherosclerosis and its complications, even though it appears to be a strong independent predictor of cardiovascular events.

\section{A. TNF- $\alpha$}

In a study from the secondary prevention cholesterol and recurrent events (CARE) trial, TNF- $\alpha$ has been associated with an elevated risk of recurrent $\mathrm{MI}$ and cardiovascular death after a first MI (570). TNF- $\alpha$ levels are correlated with ankle-brachial index, used to predict the severity of peripheral arterial disease (91) and also correlate with the burden of atherosclerosis as assessed by carotid ultrasound among healthy middle-aged men (641). However, other investigators have suggested that sTNFR levels may be a better marker of atherosclerotic burden 
than TNF- $\alpha$ itself. A study that sought to determine whether TNF- $\alpha$ and TNFR levels were associated with carotid plaque thickness concluded that relative elevation in TNFR levels, but not TNF- $\alpha$, was associated with carotid atherosclerosis among individuals aged $<70$ yr (112).

\section{B. IL-2}

A transient burst of T-cell activation has been detected in patients with UA $(101,103,497)$. Furthermore, patients with UA are characterized by a perturbation of the functional T-cell repertoire with a bias toward IFN- $\gamma$ production (409). In an attempt to determine the relationship between T-lymphocyte activation and CAD, plasma levels of IL-2 have been measured in coronary patients. Surprisingly, high levels of IL-2 and soluble IL-2 receptor were found in those with stable but not UA (638).

\section{IL-6}

IL-6 levels appear to be predictive of future CAD (270) and are elevated in patients with UA compared with those with stable angina (50). Patients with persistently elevated IL-6 levels demonstrate a worse in-hospital outcome following admission with UA (49). Raised levels of IL-6 are often found correlated to CRP levels, consistent with IL-6 being the main stimulant for the hepatic production of CRP (reviewed in Ref. 747).

In the fast revascularization during instability in coronary artery disease (FRISC) II trial, IL-6 was an independent predictor of mortality among patients presenting with ACS, even when measurements with a hsCRP method were included in the analysis (403). Interestingly, elevated IL-6 levels appeared to have utility in terms of directing subsequent care. Early invasive strategy in patients with elevated IL-6 levels led to a dramatic 65\% relative reduction in mortality at $1 \mathrm{yr}$ (403). In contrast, among patients with lower levels of IL-6, randomization to an early invasive strategy did not confer any benefit over a conservative strategy. This illustrates how inflammatory biomarkers can be used for risk stratification and also to identify those patients who might benefit from targeted interventional therapy.

\section{IL-7}

A role for IL-7 has been suggested in the promotion of clinical instability in CAD (152). This is based on the fact that IL-7 plasma levels were significantly increased in patients with stable angina and UA compared with healthy controls. Increased release from activated platelets appeared to be a major contributor to raised IL-7 levels in patients with CAD. In addition, IL-7 enhanced the expression of several inflammatory chemokines in peripheral blood mononuclear cells in both healthy subjects and patients with $\mathrm{CAD}$, and aspirin reduced both spontaneous and stimulated release of IL-7 from platelets (152).

\section{E. IL-8}

The prospective EPIC-Norfolk population study provided evidence that elevated plasma levels of IL-8 were associated with an increased risk of CAD in apparently healthy individuals (65). This relationship was independent of traditional cardiovascular risk factors and also independent of CRP levels. An earlier study also showed that IL-8 levels may be useful clinical predictors of unstable CAD (574).

\section{F. IL-18}

Consistent with a role of IL-18 in plaque instability (435), several observational studies showed that IL-18 levels are higher among patients with UA or MI than among patients with stable angina or normal controls (439, 490, 622, 763). Of note, ratio of IL-18 to its natural inhibitor IL-18 BP were significantly higher among patients who had recent MI than among those who did not, suggesting a relation between unopposed IL-18 activity and recent MI (490). IL-18 was also identified as a strong independent predictor of death from cardiovascular causes in patients with CAD with stable or unstable angina (57). Diabetic patients with high IL-18 had a greater carotid intima-media thickness than those with normal IL-18 (31). Furthermore, numbers of carotid plaques were higher in diabetic patients with high IL-18 than in those with normal IL-18. Moreover, IL-18 is raised in heart failure patients, in whom elevations correlate with poorer cardiac functional class and higher TNF- $\alpha$ concentrations $(440,485)$. IL-18 appears likely to participate in the pathophysiology of congestive heart failure.

\section{G. SCD40L}

In light of the experimental data showing an important role of CD40/CD40L in atherosclerosis, clinical studies were carried out to evaluate the value of SCD40L as a biomarker of cardiovascular risk. It has been reported that apparently healthy women with elevated levels of CD40L have an increased risk of MI, stroke, or cardiovascular death, a finding that remained after adjustment for traditional cardiovascular risk factors (608). Furthermore, among patients with carotid atheroma, sCD40L levels may predict the presence of lipid pool on highresolution carotid magnetic resonance imaging (56). 
Platelet stimulation is the major source of circulating sCD40L, suggesting that the SCD40L levels may be of greatest predictive value among those with ACS. Consistent with this hypothesis, SCD40L levels identify patients at risk of having recurrent ischemic events $(277,693)$. The c7E3 Fab Antiplatelet Therapy in Unstable Refractory Angina (CAPTURE) study demonstrated that elevated levels of SCD40L identified the subgroup of patients with ACS who are at highest risk of death or nonfatal MI over 6-mo follow-up (277). Moreover, the risk associated with elevated sCD40 ligand levels was markedly attenuated by randomization to treatment with the glycoprotein IIb/IIIa receptor antagonist abciximab.

\section{H. IL-10}

We have seen that IL-10 is a potent antiatherogenic cytokine. Measurement of IL-10 levels has thus been sought in several clinical studies in an attempt to evaluate its value as a predictor of adverse cardiac events. Patients with UA who had cardiac events during a 3-mo follow-up period showed lower levels of IL-10 on admission when compared with patients with a noncomplicated evolution (20). In the CAPTURE trial, elevated IL-10 serum levels were associated consistently with a significantly improved outcome of patients with ACS (276). The predictive value of IL-10 serum levels was independent of elevated troponin levels. Thus a reduced IL-10 serum level is not only a marker of plaque instability favoring the development of ACS but, more importantly, is indicative of a poor prognosis even after the occurrence of an acute ischemic event caused by plaque instability. In addition, the beneficial effect of elevated serum levels of IL-10 was restricted to patients with elevated CRP serum levels indicative of an enhanced systemic inflammatory response. These data support the concept established from experimental data that the balance between pro- and anti-inflammatory cytokines is a major determinant of plaque instability and of patient outcome in ACS. In addition, increased IL-10 serum levels are associated with improved systemic endothelial vasoreactivity in patients with elevated CRP serum levels, demonstrating that the pro- and anti-inflammatory balance is a major determinant of the endothelial function (203).

\section{M-CSF}

M-CSF has emerged as one of the strongest risk factors for adverse outcomes in patients with stable angina (590). Significantly elevated M-CSF is a harbinger of ACS in these patients. M-CSF levels were significantly elevated in patients with ACS compared with patients with stable angina, the pathophysiology of which may be the aforementioned SMC loss caused by the activation of
MMPs in the plaque. Serum M-CSF levels determined $6 \mathrm{wk}$ after discharge in patients with severe unstable angina were strong predictors of cardiac events during a 2-yr follow-up (558). In contrast, admission or discharge cytokine values were not predictive of long-term outcome.

\section{THERAPEUTIC POTENTIAL}

The inflammatory nature of atherosclerosis has prompted efforts to prevent development and/or progression of disease by targeting inflammatory mediators, including cytokines, chemokines, and MMPs. However, given the long-life evolution of the disease, the benefit of such approaches is likely to be lost after the withdrawal of treatment, implying a need for the indefinite drug administration, with the attendant risks of chronic adverse side-effects, including immunosuppression. Based on our current knowledge of the role of cytokines in the disease, we would like, in conclusion, to propose some novel therapeutic strategies to combat atherosclerosis.

\section{A. Use of Anticytokines}

Several natural endogenous inhibitors of IL-1, IL-18, and TNF- $\alpha$ have been identified; these include IL-1ra, soluble IL-1 receptors, IL-18BP, and soluble TNF- $\alpha$ receptors. Although increased levels of these natural inhibitors usually occur in sera and at sites of inflammation in patients with inflammatory diseases, there might be locally an excess of these cytokines compared with their respective natural inhibitors that favors their proinflammatory action. Therefore, a potential therapeutic maneuver for treating atherosclerosis is to neutralize these implicated cytokines. Biologic agents aimed at inhibiting the proinflammatory activities of these cytokines thus far have included cytokine receptor antagonists, anticytokine monoclonal antibodies, and fusion molecules consisting of soluble cytokine receptors combined with human fusion protein constructs or polyethylene glycol. A successful example of this approach is Etanercept, a soluble TNFR fusion protein, the use of which has been shown to be effective and safe in rheumatoid arthritis (reviewed in Ref. 518). Nevertheless, blocking the bioactivity of proinflammatory cytokines, crucial activators of host defense, has proven to be accompanied by an increased susceptibility to infections. Caution is therefore warranted when these treatments are given to patients.

\section{B. Targetting Downstream Inflammasome}

The molecular characterization of the molecular complex, inflammasome, that activates the inflammatory caspase- 1 and caspase- 5 , opens the door to new therapeu- 
tic approaches for the treatment of autoinflammatory disorders, characterized by recurrent inflammatory episodes not mediated by autoantibodies or antigen-specific T cells (297), by reducing IL- $1 \beta$ and IL-18 production, because of the key roles of these two cytokines in many inflammatory diseases, including atherosclerosis. Orally active inhibitors of caspase-1, including pralnacasan, are in clinical trials in patients with rheumatoid arthritis, and decreased disease activity has been observed, particularly in patients with elevated CRP levels (559). Treating inflammation with an orally active, highly specific anticytokine agent holds considerable promise in inflammatory diseases like atherosclerosis.

\section{Targetting the JAK/STAT Pathway}

A selective JAK3 antagonist, designated CP-690 550, has recently been developed (115). As predicted by studies in humans with mutations of JAK3 and its associated receptor subunits, the drug is a potent immunosuppressant. An important feature of this drug is that it has selectivity for JAK3 and does not induce unacceptable anemia, leukopenia, or thrombocytopenia, which would be indicative of substantial JAK2 inhibition. Because JAK3 has limited tissue expression, and its only meaningful biological function is restricted to immune cells, a JAK3 antagonist is not associated with widespread effects in other organs; in this way, the selective JAK3 antagonist distinguishes itself from other immunosuppressants. The drug is effective in a preclinical model of renal allotransplantation in nonhuman primates (73). As the drug moves towards clinical trials in humans, it will be important to determine its value in atherosclerosis.

\section{Activation of the Natural Anti-inflammatory Intracellular Pathway (SOCS)}

SOCS1, when overexpressed, can inhibit signals from most hematopoietic and inflammatory cytokines that utilize the JAK/STAT pathway. Thus, although the main physiological role of SOCS1 appears to be to control IFN- $\gamma$, this promiscuous activity implies that SOCS1 agonists or mimetics might also prove beneficial in the control of inflammation mediated by multiple cytokines as occurred in atherosclerosis. In a murine model of inflammatory arthritis, overexpression of SOCS3 by periarticular injection of a SOCS3 adenovirus reduced the severity of inflammation and joint damage (635). SOCS3 is also highly expressed in inflamed intestinal mucosa of patients with ulcerative colitis and Crohn's disease (415, 657). Such diseases are driven by excessive STAT3 activation. It is postulated that SOCS3 is a negative regulator of inflammation in these diseases and that SOCS3 agonists will reduce bowel inflammation. This suggests that small molecule effectors of SOCS3 activity might be of great interest in the treatment of atherosclerosis.

\section{E. Stimulation of Treg Cells}

An existing approach for the treatment of atherosclerosis results from the identification of circulating autoantibodies against oxidized LDL in humans (526) and from the observation that an immunization with oxidized LDL significantly reduces atherosclerosis. The atheroprotective effect of this approach is mediated through the induction of antibodies against oxidized epitopes of oxLDL. Specific immunoreactive antigenic epitopes in the apolipoprotein B-100 component of LDL have been recently identified (210), and experimental observations have provided a proof of concept that active vaccination using these antigenic epitopes may represent a novel therapeutic approach for the prevention and treatment of atherosclerosis $(599,624)$.

In light of our recent findings on the role of Treg cells in atherosclerosis $(8,437)$, treatments aimed at promoting Treg cell generation such as $\mathrm{Tr} 1$ cells, $\mathrm{CD} 4{ }^{+} \mathrm{CD} 25^{+}$cells, or Th3 cells can represent an attractive tool for treating and/or preventing atherosclerosis. This might be accomplished by promoting a regulatory immune response distinct from the humoral response that generates anti-oxLDL antibodies, which would limit the risk of inflammatory complications associated with the induction of autoantibodies.

Of note, studies in nonobese (NOD) diabetic mice indicate that short-term treatment with monoclonal antibodies against CD3 induces long-term remission of established diabetes $(118,119)$ through the induction of $\mathrm{CD}^{+}{ }^{+} \mathrm{CD} 25^{+}$Treg cells (44). The CD3-specific monoclonal antibodies used in these studies recognize the $\epsilon$-chain of the CD3 complex, which is associated with the TCR for antigen recognition (117). The precise mechanisms involved in the CD3-specific antibody-induced $\mathrm{CD} 4{ }^{+} \mathrm{CD} 25^{+}$Treg cells are unknown, but Treg cells from mice treated with CD3-specific antibodies produce high levels of TGF- $\beta$, and in vivo neutralization of TGF- $\beta$ prevents the remission of autoimmune diabetes in NOD mice (44). Interestingly, use of a humanized antiCD3 antibody in patients with recent-onset type 1 diabetes shows that short-term treatment preserves residual $\beta$-cell function for at least 18 mo (333). Induction of TGF- $\beta$-dependent Treg cells by using anti-CD3 antibodies may limit the development and progression of atherosclerosis in patients with high cardiovascular risk factors.

An alternative way to induce and maintain immune tolerance in atherosclerosis would be to use peptidebased therapeutic vaccines (371). Prolonged subcutaneous infusion of low doses of peptides can transform mature $\mathrm{T}$ cells into $\mathrm{CD} 4^{+} \mathrm{CD} 25^{+}$Treg cells, persisting for 
long periods of time, even in the absence of antigen, and capable of stimulating specific immunological tolerance upon encounter with antigen (24). Such strategy of delivering low doses of specific peptides for a long period of time has been successfully used in experimental models of allergy $(84,290)$. Also, peptide derived from HSPs for the treatment of diabetes and rheumatoid arthritis has provided encouraging results $(550,561)$. This prompted us to evaluate the value of therapeutic vaccination using specific atherosclerotic plaque peptides in murine models of atherosclerosis.

\section{F. Stimulation of Macrophage Emigration From Atherosclerotic Lesions}

It had been proposed by the beginning of the 1980s that lipid-laden foam cells can migrate back from the intima into the bloodstream by crossing the arterial endothelium (229). Yet the molecular mechanisms responsible for macrophage emigration from the atherosclerotic plaque were totally unknown. Of note, recent in vitro and in vivo studies exploring the mechanisms of monocyte egress from the vessel wall revealed that PAF and lysophosphatidic acid (LPA) inhibit monocyte transmigration (410). Moreover, cells that emigrate from atherosclerotic lesions to lymphoid organs, after transplantation of an atherosclerotic aortic segment from an $\mathrm{apoE}^{-/-}$donor to a C57BL/6 recipent, express high levels of the major histocompatibility complex class II molecules I-Ab, CD11b, and the M-CSF receptor CD115. Clearance of monocytes from the atherosclerotic plaque by conversion into migratory cells using a specific set of cytokines may bolster plaque regression.

\section{CONCLUSION}

Much has been learned about the role of cytokines in atherosclerosis since their presence in the human atherosclerotic plaque was first discovered over 25 years ago. Proinflammatory cytokines stimulate chemokines and adhesion molecules, leading to early recruitment of monocytes and lymphocytes in the intima. Furthermore, cytokines exert potential noxious effects in late atherosclerosis when they activate MMPs in macrophages and vascular cells and promote cell apoptosis, resulting in weakened plaques that are more prone to rupture or erosion. The balance between pro- and anti-inflammatory cytokines has emerged as a major determinant of plaque stability. Nonetheless, many aspects of plaque formation and evolution remain unresolved. For one, most if not all of our understanding of the molecular mechanisms of atherogenesis are based on experiments in murine models of atherosclerosis. To what extent can the findings obtained in $\mathrm{apoE}^{-/-}$or $\mathrm{LDLr}^{-/-}$mice be translated to human atherosclerosis? Inasmuch as no model can reproduce plaque rupture or plaque erosion in humans, how can the importance of cytokines be evaluated in these settings? Atherosclerosis definitely proceeds from a local inflammatory process. Yet, in atherosclerosis increased expression of cytokines in the plaque usually parallels that in spleen cells $(541,572,791)$. Is atherosclerosis then really a local or a systemic inflammatory disease (422)?

The upsurge in our understanding of the role played by inflammation in atherosclerosis has significant implications for current and future therapeutic approaches for primary and secondary prevention of atherothrombotic events. The development of new treatments will focus on strategies that decrease the inflammatory response and tip the balance in favor of anti-inflammatory mediators and, therefore, plaque stability. However, crucial questions still arise: Are there cytokines that are more specific of atherosclerosis? Do they have site specificity: coronary versus carotid versus peripheral arteries? Do cytokines play a similar role in early and late atherosclerosis? We have seen that future therapeutic approaches may include agents that block proinflammatory cytokine signaling, agents that augment the anti-inflammatory activity of other cytokines, and agents that either block the transcription of inflammatory mediating molecules or upregulate anti-inflammatory molecules. Yet, when and how long to treat patients with $\mathrm{CAD}$ with such agents that trigger the cytokine network remains to be resolved.

\section{ACKNOWLEDGMENTS}

We thank Stéphanie Lehoux for critical reading of the manuscript and for the artwork. We apologize to those individuals whose work was not cited.

Addresss for reprint requests and other correspondence: A. Tedgui, INSERM U. 689, 41 bd. de la Chapelle, 75475 Paris Cedex 10, France (e-mail: tedgui@larib.inserm.fr).

\section{GRANTS}

This work is supported by the European Vascular Genomics Network (EVGN; http://www.evgn.org), a Network of Excellence funded by the European Community's Sixth Framework Program for Research Priority 1 "Life sciences, genomics, and biotechnology for health" (contract no. LSHM-CT-2003-503254).

\section{REFERENCES}

1. AbdAlla S, Lother H, Langer A, el Faramawy Y, and Quitterer U. Factor XIIIA transglutaminase crosslinks AT1 receptor dimers of monocytes at the onset of atherosclerosis. Cell 119: 343-354, 2004.

2. Adams CW, Morgan RS, and Bayliss OB. No regression of atheroma over one year in rabbits previously fed a cholesterol-enriched diet. Atherosclerosis 18: 429-444, 1973.

3. Agostini L, Martinon F, Burns K, McDermott MF, Hawkins PN, and Tschopp J. NALP3 forms an IL-1beta-processing inflammasome with increased activity in Muckle-Wells autoinflammatory disorder. Immunity 20: 319-325, 2004.

4. Ahmed Z, Ravandi A, Maguire GF, Kuksis A, and Connelly PW. Formation of apolipoprotein AI-phosphatidylcholine core al- 
dehyde Schiff base adducts promotes uptake by THP-1 macrophages. Cardiovasc Res 58: 712-720, 2003.

5. Aicher A, Heeschen C, Mildner-Rihm C, Urbich C, Ihling C, Technau-Ihling K, Zeiher AM, and Dimmeler S. Essential role of endothelial nitric oxide synthase for mobilization of stem and progenitor cells. Nat Med 9: 1370-1376, 2003.

6. Aiello RJ, Bourassa PA, Lindsey S, Weng W, Freeman A, and Showell HJ. Leukotriene $\mathrm{B}_{4}$ receptor antagonism reduces monocytic foam cells in mice. Arterioscler Thromb Vasc Biol 22: 443449, 2002.

7. Aikawa M, Rabkin E, Okada Y, Voglic SJ, Clinton SK, Brinckerhoff CE, Sukhova GK, and Libby P. Lipid lowering by diet reduces matrix metalloproteinase activity and increases collagen content of rabbit atheroma: a potential mechanism of lesion stabilization. Circulation 97: 2433-2444, 1998.

8. Ait Oufella H, Salomon BL, Potteaux S, Robertson AK, Gourdy P, Merval R, Esposito B, Cohen JL, Fisson S, Flavell RA, Hansson GK, Klatzmann D, Tedgui A, and Mallat Z. Natural regulatory $\mathrm{T}$ cells control the development of atherosclerosis in mice. Nat Med. In press.

9. Akbari O, DeKruyff RH, and Umetsu DT. Pulmonary dendritic cells producing IL-10 mediate tolerance induced by respiratory exposure to antigen. Nat Immunol 2: 725-731, 2001.

10. Albert ML. Death-defying immunity: do apoptotic cells influence antigen processing and presentation? Nat Rev Immunol 4: 223-231, 2004 .

11. Albert ML, Kim JI, and Birge RB. Alphavbeta5 integrin recruits the CrkII-Dock180-rac1 complex for phagocytosis of apoptotic cells. Nat Cell Biol 2: 899-905, 2000.

12. Albert ML, Sauter B, and Bhardwaj N. Dendritic cells acquire antigen from apoptotic cells and induce class I-restricted CTLs. Nature 392: 86-89, 1998.

13. Alexopoulou L, Holt AC, Medzhitov R, and Flavell RA. Recognition of double-stranded RNA and activation of NF-kappaB by Toll-like receptor 3. Nature 413: 732-738, 2001.

14. Almeida GD, Porada CD, Stjeor S, and Ascensao JL. Human cytomegalovirus alters interleukin-6 production by endothelial cells. Blood 83: 370-376, 1994.

15. Alvarez A, Cerda-Nicolas M, Naim Abu Nabah Y, Mata M, Issekutz AC, Panes J, Lobb RR, and Sanz MJ. Direct evidence of leukocyte adhesion in arterioles by angiotensin II. Blood 104: 402-408, 2004.

16. Ameli S, Hultgardhnilsson A, Regnstrom J, Calara F, Yano J, Cercek B, Shah PK, and Nilsson J. Effect of immunization with homologous LDL and oxidized LDL on early atherosclerosis in hypercholesterolemic rabbits. Arterioscler Thromb Vasc Biol 16: 1074-1079, 1996.

17. Amento EP, Ehsani N, Palmer H, and Libby P. Cytokines and growth factors positively and negatively regulate interstitial collagen gene expression in human vascular smooth muscle cells. Arterioscler Thromb 11: 1223-1230, 1991.

18. Ameziane N, Beillat T, Verpillat P, Chollet-Martin S, Aumont MC, Seknadji P, Lamotte M, Lebret D, Ollivier V, and de Prost D. Association of the Toll-like receptor 4 gene Asp299Gly polymorphism with acute coronary events. Arterioscler Thromb Vasc Biol 23: e61-e64, 2003.

19. Andre P, Prasad KS, Denis CV, He M, Papalia JM, Hynes RO, Phillips DR, and Wagner DD. CD40L stabilizes arterial thrombi by a beta3 integrin-dependent mechanism. Nat Med 8: 247-252, 2002.

20. Anguera I, Miranda-Guardiola F, Bosch X, Filella X, Sitges M, Marin JL, Betriu A, and Sanz G. Elevation of serum levels of the anti-inflammatory cytokine interleukin-10 and decreased risk of coronary events in patients with unstable angina. Am Heart $J$ 144: 811-817, 2002.

21. Anitschkow $\mathbf{N}$ and Chalatov $\mathbf{S}$. Ueber experimentalle Cholesterinsteatose. Zbl allg Path path Anat 24: 1-9, 1913.

22. Apostolopoulos J, Davenport P, and Tipping PG. Interleukin-8 production by macrophages from atheromatous plaques. Arterioscler Thromb Vasc Biol 16: 1007-1012, 1996.

23. Apostolou I, Sarukhan A, Klein L, and von Boehmer H. Origin of regulatory T cells with known specificity for antigen. Nat Immunol 3: 756-763, 2002.
24. Apostolou I and von Boehmer H. In vivo instruction of suppressor commitment in naive T cells. J Exp Med 199: 1401-1408, 2004.

25. Aprahamian T, Rifkin I, Bonegio R, Hugel B, Freyssinet JM, Sato K, Castellot JJ Jr, and Walsh K. Impaired clearance of apoptotic cells promotes synergy between atherogenesis and autoimmune disease. $J$ Exp Med 199: 1121-1131, 2004.

26. Arbustini E, Grasso M, Diegoli M, Pucci A, Bramerio M, Ardissino D, Angoli L, de Servi S, Bramucci E, and Mussini A. Coronary atherosclerotic plaques with and without thrombus in ischemic heart syndromes: a morphologic, immunohistochemical, and biochemical study. Am J Cardiol 68: 36B-50B, 1991.

27. Argmann CA, Van Den Diepstraten CH, Sawyez CG, Edwards JY, Hegele RA, Wolfe BM, and Huff MW. Transforming growth factor-beta1 inhibits macrophage cholesteryl ester accumulation induced by native and oxidized VLDL remnants. Arterioscler Thromb Vasc Biol 21: 2011-2018, 2001.

28. Asahara T, Murohara T, Sullivan A, Silver M, Vanderzee R, Li T, Witzenbichler B, Schatteman G, and Isner JM. Isolation of putative progenitor endothelial cells for angiogenesis. Science 275: 964-967, 1997.

29. Ashkar S, Weber GF, Panoutsakopoulou V, Sanchirico ME, Jansson M, Zawaideh S, Rittling SR, Denhardt DT, Glimcher MJ, and Cantor H. Eta-1 (osteopontin): an early component of type-1 (cell-mediated) immunity. Science 287: 860-864, 2000.

30. Aslanian AM, Chapman HA, and Charo IF. Transient role for CD1d-restricted natural killer $\mathrm{T}$ cells in the formation of atherosclerotic lesions. Arterioscler Thromb Vasc Biol 25: 628-632, 2005.

31. Aso Y, Okumura K, Takebayashi K, Wakabayashi S, and Inukai T. Relationships of plasma interleukin-18 concentrations to hyperhomocysteinemia and carotid intimal-media wall thickness in patients with type 2 diabetes. Diabetes Care 26: 2622-2627, 2003.

32. Asseman C, Mauze S, Leach MW, Coffman RL, and Powrie F. An essential role for interleukin 10 in the function of regulatory $\mathrm{T}$ cells that inhibit intestinal inflammation. J Exp Med 190: 995-1004, 1999.

33. Balkwill FR and Burke F. The cytokine network. Immunol Today 10: 299-304, 1989.

34. Banchereau J, Briere F, Caux C, Davoust J, Lebecque S, Liu YJ, Pulendran B, and Palucka K. Immunobiology of dendritic cells. Annu Rev Immunol 18: 767-811, 2000.

35. Barath P, Fishlein MC, Cao J, Berenson J, Helfant RH, and Forrester JS. Detection and localization of tumor necrosis factor in human atheroma. Am J Cardiol 65: 297-302, 1990.

36. Barry OP, Pratico D, Savani RC, and FitzGerald GA. Modulation of monocyte-endothelial cell interactions by platelet microparticles. J Clin Invest 102: 136-144, 1998.

37. Barton BE. The biological effects of interleukin 6. Med Res Rev 16: 87-109, 1996.

38. Basta G, Lazzerini G, Del Turco S, Ratto GM, Schmidt AM, and De Caterina $\mathbf{R}$. At least 2 distinct pathways generating reactive oxygen species mediate vascular cell adhesion molecule-1 induction by advanced glycation end products. Arterioscler Thromb Vasc Biol 25: 1401-1407, 2005.

39. Basta G, Lazzerini G, Massaro M, Simoncini T, Tanganelli P, Fu C, Kislinger T, Stern DM, Schmidt AM, and De Caterina R. Advanced glycation end products activate endothelium through signal-transduction receptor RAGE: a mechanism for amplification of inflammatory responses. Circulation 105: 816-822, 2002

40. Bavendiek U, Zirlik A, LaClair S, MacFarlane L, Libby P, and Schonbeck U. Atherogenesis in mice does not require CD40 ligand from bone marrow-derived cells. Arterioscler Thromb Vasc Biol 25: 1244-1249, 2005

41. Bayraktutan U, Draper N, Lang D, and Shah AM. Expression of functional neutrophil-type NADPH oxidase in cultured rat coronary microvascular endothelial cells. Cardiovasc Res 38: 256-262, 1998.

42. Bazan JF, Bacon KB, Hardiman G, Wang W, Soo K, Rossi D, Greaves DR, Zlotnik A, and Schall TJ. A new class of membrane-bound chemokine with a CX3C motif. Nature 385: 640-644, 1997.

43. Bazzoni G and Dejana E. Endothelial cell-to-cell junctions: molecular organization and role in vascular homeostasis. Physiol Rev 84: 869-901, 2004. 
44. Belghith M, Bluestone JA, Barriot S, Megret J, Bach JF, and Chatenoud L. TGF-beta-dependent mechanisms mediate restoration of self-tolerance induced by antibodies to CD3 in overt autoimmune diabetes. Nat Med 9: 1202-1208, 2003.

45. Bennett CL, Christie J, Ramsdell F, Brunkow ME, Ferguson PJ, Whitesell L, Kelly TE, Saulsbury FT, Chance PF, and Ochs HD. The immune dysregulation, polyendocrinopathy, enteropathy, $\mathrm{X}$-linked syndrome (IPEX) is caused by mutations of FOXP3. Nat Genet 27: 20-21, 2001.

46. Berk BC, Weintraub WS, and Alexander RW. Elevation of C-reactive protein in "active" coronary artery disease. Am J Cardiol 65: 168-172, 1990.

47. Bevilacqua MP, Stengelin S, Gimbrone MA Jr, and Seed B. Endothelial leukocyte adhesion molecule 1: an inducible receptor for neutrophils related to complement regulatory proteins and lectins. Science 243: 1160-1165, 1989.

48. Bhakdi S, Torzewski M, Paprotka K, Schmitt S, Barsoom H, Suriyaphol P, Han SR, Lackner KJ, and Husmann M. Possible protective role for C-reactive protein in atherogenesis: complement activation by modified lipoproteins halts before detrimental terminal sequence. Circulation 109: 1870-1876, 2004.

49. Biasucci LM, Liuzzo G, Fantuzzi G, Caligiuri G, Rebuzzi AG, Ginnetti F, Dinarello CA, and Maseri A. Increasing levels of interleukin (IL)-1Ra and IL-6 during the first 2 days of hospitalization in unstable angina are associated with increased risk of inhospital coronary events. Circulation 99: 2079-2084, 1999.

50. Biasucci LM, Vitelli A, Liuzzo G, Altamura S, Caligiuri G, Monaco C, Rebuzzi AG, Ciliberto G, and Maseri A. Elevated levels of interleukin-6 in unstable angina. Circulation 94: 874-877, 1996.

51. Bierhaus A, Chevion S, Chevion M, Hofmann M, Quehenberger $\mathbf{P}$, Illmer $\mathbf{T}$, Luther $\mathbf{T}$, Berentshtein $\mathbf{E}$, Tritschler $\mathbf{H}$, Muller M, Wahl P, Ziegler R, and Nawroth PP. Advanced glycation end product-induced activation of NF-kappaB is suppressed by alpha-lipoic acid in cultured endothelial cells. Diabetes 46: 14811490, 1997.

52. Binder CJ, Chang MK, Shaw PX, Miller YI, Hartvigsen K, Dewan A, and Witztum JL. Innate and acquired immunity in atherogenesis. Nat Med 8: 1218-1226, 2002.

53. Binder CJ, Hartvigsen K, Chang MK, Miller M, Broide D, Palinski W, Curtiss LK, Corr M, and Witztum JL. IL-5 links adaptive and natural immunity specific for epitopes of oxidized LDL and protects from atherosclerosis. J Clin Invest 114: 427-437, 2004.

54. Bjorkbacka H, Kunjathoor VV, Moore KJ, Koehn S, Ordija CM, Lee MA, Means T, Halmen K, Luster AD, Golenbock DT, and Freeman MW. Reduced atherosclerosis in MyD88-null mice links elevated serum cholesterol levels to activation of innate immunity signaling pathways. Nat Med 10: 416-421, 2004.

55. Black RA, Rauch CT, Kozlosky CJ, Peschon JJ, Slack JL, Wolfson MF, Castner BJ, Stocking KL, Reddy P, Srinivasan S, Nelson N, Boiani N, Schooley KA, Gerhart M, Davis R, Fitzner JN, Johnson RS, Paxton RJ, March CJ, and Cerretti DP. A metalloproteinase disintegrin that releases tumour-necrosis factoralpha from cells. Nature 385: 729-733, 1997.

56. Blake GJ and Ridker PM. C-reactive protein and other inflammatory risk markers in acute coronary syndromes. $J$ Am Coll Cardiol 41: 37S-42S, 2003.

57. Blankenberg S, Tiret L, Bickel C, Peetz D, Cambien F, Meyer $\mathbf{J}$, and Rupprecht HJ. Interleukin-18 is a strong predictor of cardiovascular death in stable and unstable angina. Circulation 106: 24-30, 2002.

58. Bobik A, Agrotis A, Kanellakis P, Dilley R, Krushinsky A, Smirnov V, Tararak E, Condron M, and Kostolias G. Distinct patterns of transforming growth factor-beta isoform and receptor expression in human atherosclerotic lesions. Colocalization implicates TGF-beta in fibrofatty lesion development. Circulation 99: 2883-2891, 1999.

59. Bobryshev YV. Dendritic cells and their involvement in atherosclerosis. Curr Opin Lipidol 11: 511-517, 2000.

60. Bobryshev YV. Dendritic cells in atherosclerosis: current status of the problem and clinical relevance. Eur Heart J 26: 1700-1704, 2005 .
61. Bochkov VN, Kadl A, Huber J, Gruber F, Binder BR, and Leitinger $\mathbf{N}$. Protective role of phospholipid oxidation products in endotoxin-induced tissue damage. Nature 419: 77-81, 2002.

62. Bochkov VN, Mechtcheriakova D, Lucerna M, Huber J, Malli R, Graier WF, Hofer E, Binder BR, and Leitinger N. Oxidized phospholipids stimulate tissue factor expression in human endothelial cells via activation of ERK/EGR-1 and $\mathrm{Ca}(++) / \mathrm{NFAT}$. Blood 99: 199-206, 2002.

63. Bodary PF, Gu S, Shen Y, Hasty AH, Buckler JM, and Eitzman DT. Recombinant leptin promotes atherosclerosis and thrombosis in apolipoprotein E-deficient mice. Arterioscler Thromb Vasc Biol 25: e119-e122, 2005.

64. Boekholdt SM, Agema WR, Peters RJ, Zwinderman AH, van der Wall EE, Reitsma PH, Kastelein JJ, and Jukema JW. Variants of toll-like receptor 4 modify the efficacy of statin therapy and the risk of cardiovascular events. Circulation 107: 2416-2421, 2003.

65. Boekholdt SM, Peters RJ, Hack CE, Day NE, Luben R, Bingham SA, Wareham NJ, Reitsma PH, and Khaw KT. IL-8 plasma concentrations and the risk of future coronary artery disease in apparently healthy men and women: the EPIC-Norfolk prospective population study. Arterioscler Thromb Vasc Biol 24: 1503-1508, 2004.

66. Boerth NJ, Dey NB, Cornwell TL, and Lincoln TM. Cyclic GMP-dependent protein kinase regulates vascular smooth muscle cell phenotype. J Vasc Res 34: 245-259, 1997.

67. Boesten LS, Zadelaar AS, van Nieuwkoop A, Gijbels MJ, de Winther MP, Havekes LM, and van Vlijmen BJ. Tumor necrosis factor-alpha promotes atherosclerotic lesion progression in APOE*3-Leiden transgenic mice. Cardiovasc Res 66: 179-185, 2005.

68. Bogdan C, Vodovotz Y, and Nathan C. Macrophage deactivation by interleukin-10. J Exp Med 174: 1549-1555, 1991.

69. Boisvert WA, Santiago R, Curtiss LK, and Terkeltaub RA. A leukocyte homologue of the IL-8 receptor CXCR-2 mediates the accumulation of macrophages in atherosclerotic lesions of LDL receptor-deficient mice. J Clin Invest 101: 353-363, 1998.

70. Bokarewa M, Nagaev I, Dahlberg L, Smith U, and Tarkowski A. Resistin, an adipokine with potent proinflammatory properties. J Immunol 174: 5789-5795, 2005.

71. Bonavida B, Mencia-Huerta JM, and Braquet P. Effect of platelet-activating factor on monocyte activation and production of tumor necrosis factor. Int Arch Allergy Appl Immunol 88: 157-160, 1989.

72. Bonow RO, Smaha LA, Smith SC Jr, Mensah GA, and Lenfant C. World Heart Day 2002: the international burden of cardiovascular disease: responding to the emerging global epidemic. Circulation 106: 1602-1605, 2002.

73. Borie DC, Changelian PS, Larson MJ, Si MS, Paniagua R, Higgins JP, Holm B, Campbell A, Lau M, Zhang S, Flores MG, Rousvoal G, Hawkins J, Ball DA, Kudlacz EM, Brissette WH, Elliott EA, Reitz BA, and Morris RE. Immunosuppression by the JAK3 inhibitor CP-690,550 delays rejection and significantly prolongs kidney allograft survival in nonhuman primates. Transplantation 79: 791-801, 2005.

74. Boring L, Gosling J, Cleary M, and Charo IF. Decreased lesion formation in CCR2-/- mice reveals a role for chemokines in the initiation of atherosclerosis. Nature 394: 894-897, 1998.

75. Botto M, Dell'Agnola C, Bygrave AE, Thompson EM, Cook HT, Petry F, Loos M, Pandolfi PP, and Walport MJ. Homozygous C1q deficiency causes glomerulonephritis associated with multiple apoptotic bodies. Nat Genet 19: 56-59, 1998.

76. Boulanger CM, Scoazec A, Ebrahimian T, Henry P, Mathieu E, Tedgui A, and Mallat $\mathbf{Z}$. Circulating microparticles from patients with myocardial infarction cause endothelial dysfunction. Circulation 104: 2649-2652, 2001.

77. Boulay JL, O'Shea JJ, and Paul WE. Molecular phylogeny within type I cytokines and their cognate receptors. Immunity 19: 159 163, 2003.

78. Bourcier T, Sukhova G, and Libby P. The nuclear factor kappa-B signaling pathway participates in dysregulation of vascular smooth muscle cells in vitro and in human atherosclerosis. $J$ Biol Chem 272: 15817-15824, 1997. 
79. Boyle JJ, Weissberg PL, and Bennett MR. Human macrophageinduced vascular smooth muscle cell apoptosis requires $\mathrm{NO}$ enhancement of Fas/Fas-L interactions. Arterioscler Thromb Vasc Biol 22: 1624-1630, 2002.

80. Boyle JJ, Weissberg PL, and Bennett MR. Tumor necrosis factor-alpha promotes macrophage-induced vascular smooth muscle cell apoptosis by direct and autocrine mechanisms. Arterioscler Thromb Vasc Biol 23: 1553-1558, 2003.

81. Brand K, Mackman N, and Curtiss LK. Interferon-gamma inhibits macrophage apolipoprotein $\mathrm{E}$ production by posttranslational mechanisms. J Clin Invest 91: 2031-2039, 1993.

82. Brand K, Page S, Rogler G, Bartsch A, Brandl R, Knuechel R, Page M, Kaltschmidt C, Baeuerle PA, and Neumeier D. Activated transcription factor nuclear factor-kappa $B$ is present in the atherosclerotic lesion. J Clin Invest 97: 1715-1722, 1996.

83. Branen L, Hovgaard L, Nitulescu M, Bengtsson E, Nilsson J, and Jovinge $\mathbf{S}$. Inhibition of tumor necrosis factor-alpha reduces atherosclerosis in apolipoprotein E knockout mice. Arterioscler Thromb Vasc Biol 24: 2137-2142, 2004.

84. Briner TJ, Kuo MC, Keating KM, Rogers BL, and Greenstein JL. Peripheral T-cell tolerance induced in naive and primed mice by subcutaneous injection of peptides from the major cat allergen Feld I. Proc Natl Acad Sci USA 90: 7608-7612, 1993.

85. Briscoe DM, Cotran RS, and Pober JS. Effects of tumor necrosis factor, lipopolysaccharide, and IL-4 on the expression of vascular cell adhesion molecule-1 in vivo: correlation with cd3+ T-cell infiltration. J Immunol 149: 2954-2960, 1992.

86. Brizzi MF, Formato L, Dentelli P, Rosso A, Pavan M, Garbarino G, Pegoraro M, Camussi G, and Pegoraro L. Interleukin-3 stimulates migration and proliferation of vascular smooth muscle cells: a potential role in atherogenesis. Circulation 103: 549-554, 2001.

87. Brown MS and Goldstein JL. Lipoprotein metabolism in the macrophage: implications for cholesterol deposition in atherosclerosis. Annu Rev Biochem 52: 223-261, 1983.

88. Brown MS and Goldstein JL. A receptor-mediated pathway for cholesterol homeostasis. Science 232: 34-47, 1986.

89. Bruemmer D, Collins AR, Noh G, Wang W, Territo M, AriasMagallona S, Fishbein MC, Blaschke F, Kintscher U, Graf K, Law RE, and Hsueh WA. Angiotensin II-accelerated atherosclerosis and aneurysm formation is attenuated in osteopontin-deficient mice. J Clin Invest 112: 1318-1331, 2003.

90. Bruggeman CA, Marjorie HJ, and Nelissen-Vrancken G. Cytomegalovirus and atherogenesis. Antiviral Res 43: 135-144, 1999.

91. Bruunsgaard H, Andersen-Ranberg K, Jeune B, Pedersen AN, Skinhoj P, and Pedersen BK. A high plasma concentration of TNF-alpha is associated with dementia in centenarians. J Gerontol A Biol Sci Med Sci 54: M357-364, 1999.

92. Bucciarelli LG, Wendt T, Qu W, Lu Y, Lalla E, Rong LL, Goova MT, Moser B, Kislinger T, Lee DC, Kashyap Y, Stern DM, and Schmidt AM. RAGE blockade stabilizes established atherosclerosis in diabetic apolipoprotein E-null mice. Circulation 106: 28272835, 2002.

93. Buffon A, Biasucci LM, Liuzzo G, D'Onofrio G, Crea F, and Maseri A. Widespread coronary inflammation in unstable angina. N Engl J Med 347: 5-12, 2002.

94. Buono C, Binder CJ, Stavrakis G, Witztum JL, Glimcher LH, and Lichtman AH. T-bet deficiency reduces atherosclerosis and alters plaque antigen-specific immune responses. Proc Natl Acad Sci USA 102: 1596-1601, 2005.

95. Buono C, Come CE, Stavrakis G, Maguire GF, Connelly PW, and Lichtman AH. Influence of interferon-gamma on the extent and phenotype of diet-induced atherosclerosis in the LDLR-deficient mouse. Arterioscler Thromb Vasc Biol 23: 454-460, 2003.

96. Burger PC and Wagner DD. Platelet P-selectin facilitates atherosclerotic lesion development. Blood 101: 2661-2666, 2003.

97. Burger-Kentischer A, Gobel H, Kleemann R, Zernecke A, Bucala $R$, Leng $L$, Finkelmeier $D$, Geiger G, Schaefer HE, Schober A, Weber C, Brunner H, Rutten H, Ihling C, and Bernhagen J. Reduction of the aortic inflammatory response in spontaneous atherosclerosis by blockade of macrophage migration inhibitory factor (MIF). Atherosclerosis 184: 28-38, 2006.
98. Burger-Kentischer A, Goebel H, Seiler R, Fraedrich G, Schaefer HE, Dimmeler S, Kleemann R, Bernhagen J, and Ihling C. Expression of macrophage migration inhibitory factor in different stages of human atherosclerosis. Circulation 105: 15611566, 2002.

99. Burstein SA. Platelets and cytokines. Curr Opin Hematol 1: 373380, 1994.

100. Calandra $\mathbf{T}$ and Roger T. Macrophage migration inhibitory factor: a regulator of innate immunity. Nat Rev Immunol 3: 791-800, 2003.

101. Caligiuri G, Liuzzo G, Biasucci LM, and Maseri A. Immune system activation follows inflammation in unstable angina: pathogenetic implications. J Am Coll Cardiol 32: 1295-1304, 1998.

102. Caligiuri G, Nicoletti A, Poirier B, and Hansson GK. Protective immunity against atherosclerosis carried by B cells of hypercholesterolemic mice. J Clin Invest 109: 745-753, 2002.

103. Caligiuri G, Paulsson G, Nicoletti A, Maseri A, and Hansson GK. Evidence for antigen-driven T-cell response in unstable angina. Circulation 102: 1114-1119, 2000.

104. Caligiuri G, Rudling M, Ollivier V, Jacob MP, Michel JB, Hansson GK, and Nicoletti A. Interleukin-10 deficiency increases atherosclerosis, thrombosis, and low-density lipoproteins in apolipoprotein E knockout mice. Mol Med 9: 10-17, 2003.

105. Campbell JJ and Butcher EC. Chemokines in tissue-specific and microenvironment-specific lymphocyte homing. Curr Opin Immunol 12: 336-341, 2000.

106. Campbell TC, Parpia B, and Chen J. Diet, lifestyle, and the etiology of coronary artery disease: the Cornell China study. Am J Cardiol 82: 18T-21T, 1998.

107. Canault M, Peiretti F, Mueller C, Kopp F, Morange P, Rihs S, Portugal H, Juhan-Vague I, and Nalbone G. Exclusive expression of transmembrane TNF-alpha in mice reduces the inflammatory response in early lipid lesions of aortic sinus. Atherosclerosis 172: 211-218, 2004.

108. Cannon CP, Braunwald E, McCabe CH, Grayston JT, Muhlestein B, Giugliano RP, Cairns R, and Skene AM. Antibiotic treatment of Chlamydia pneumoniae after acute coronary syndrome. N Engl J Med 352: 1646-1654, 2005.

109. Capers Qt Alexander RW, Lou P, De Leon H, Wilcox JN, Ishizaka N, Howard AB, and Taylor WR. Monocyte chemoattractant protein-1 expression in aortic tissues of hypertensive rats. Hypertension 30: 1397-1402, 1997.

110. Caramalho I, Lopes-Carvalho T, Ostler D, Zelenay S, Haury M, and Demengeot J. Regulatory T cells selectively express tolllike receptors and are activated by lipopolysaccharide. J Exp Med 197: 403-411, 2003.

111. Caro CG, Fitz-Gerald JM, and Schroter RC. Atheroma and arterial wall shear. Proc R Soc Lond B Biol Sci 117: 109-159, 1971.

112. Castellanos M, Castillo J, Garcia MM, Leira R, Serena J, Chamorro A, and Davalos A. Inflammation-mediated damage in progressing lacunar infarctions: a potential therapeutic target. Stroke 33: 982-987, 2002.

113. Cavaillon JM, Adib-Conquy M, Fitting C, Adrie C, and Payen D. Cytokine cascade in sepsis. Scand J Infect Dis 35: 535-544, 2003.

114. Cercek B, Shah PK, Noc M, Zahger D, Zeymer U, Matetzky S, Maurer G, and Mahrer P. Effect of short-term treatment with azithromycin on recurrent ischaemic events in patients with acute coronary syndrome in the Azithromycin in Acute Coronary Syndrome (AZACS) trial: a randomised controlled trial. Lancet 361: 809-813, 2003.

115. Changelian PS, Flanagan ME, Ball DJ, Kent CR, Magnuson KS, Martin WH, Rizzuti BJ, Sawyer PS, Perry BD, Brissette WH, McCurdy SP, Kudlacz EM, Conklyn MJ, Elliott EA, Koslov ER, Fisher MB, Strelevitz TJ, Yoon K, Whipple DA, Sun J, Munchhof MJ, Doty JL, Casavant JM, Blumenkopf TA, Hines M, Brown MF, Lillie BM, Subramanyam C, Shang-Poa C, Milici AJ, Beckius GE, Moyer JD, Su C, Woodworth TG, Gaweco AS, Beals CR, Littman BH, Fisher DA, Smith JF, Zagouras P, Magna HA, Saltarelli MJ, Johnson KS, Nelms LF, Des Etages SG, Hayes LS, Kawabata TT, Finco-Kent D, Baker DL, Larson M, Si MS, Paniagua R, Higgins J, Holm B, Reitz B, Zhou YJ, Morris RE, O'Shea JJ, and Borie DC. Prevention of organ allograft rejection by a specific Janus kinase 3 inhibitor. Science 302: 875-878, 2003. 
116. Chappell DC, Varner SE, Nerem RM, Medford RM, and Alexander RW. Oscillatory shear stress stimulates adhesion molecule expression in cultured human endothelium. Circ Res 82: 532-539, 1998.

117. Chatenoud L. CD3-specific antibodies restore self-tolerance: mechanisms and clinical applications. Curr Opin Immunol 2005.

118. Chatenoud L, Primo J, and Bach JF. CD3 antibody-induced dominant self tolerance in overtly diabetic NOD mice. J Immunol 158: 2947-2954, 1997.

119. Chatenoud L, Thervet E, Primo J, and Bach JF. Anti-CD3 antibody induces long-term remission of overt autoimmunity in nonobese diabetic mice. Proc Natl Acad Sci USA 91: 123-127, 1994.

120. Chen ML, Pittet MJ, Gorelik L, Flavell RA, Weissleder R, von Boehmer H, and Khazaie K. Regulatory T cells suppress tumorspecific CD8 T cell cytotoxicity through TGF-beta signals in vivo. Proc Natl Acad Sci USA 102: 419-424, 2005.

121. Chen W, Frank ME, Jin W, and Wahl SM. TGF-beta released by apoptotic $\mathrm{T}$ cells contributes to an immunosuppressive milieu. Immunity 14: 715-725, 2001.

122. Chen XL, Tummala PE, Olbrych MT, Alexander RW, and Medford RM. Angiotensin II induces monocyte chemoattractant protein-1 gene expression in rat vascular smooth muscle cells. Circ Res 83: 952-959, 1998.

123. Chen Y, Kuchroo VK, Inobe J, Hafler DA, and Weiner HL. Regulatory T cell clones induced by oral tolerance: suppression of autoimmune encephalomyelitis. Science 265: 1237-1240, 1994.

124. Chen YR, Shrivastava A, and Tan TH. Down-regulation of the c-Jun N-terminal kinase (JNK) phosphatase M3/6 and activation of JNK by hydrogen peroxide and pyrrolidine dithiocarbamate. Oncogene 20: 367-374, 2001.

125. Chen Z, Sakuma M, Zago AC, Zhang X, Shi C, Leng L, Mizue Y, Bucala R, and Simon D. Evidence for a role of macrophage migration inhibitory factor in vascular disease. Arterioscler Thromb Vasc Biol 24: 709-714, 2004.

126. Cheng JJ, Wung BS, Chao YJ, and Wang DL. Cyclic strain enhances adhesion of monocytes to endothelial cells by increasing intercellular adhesion molecule-1 expression. Hypertension 28: 386-391, 1996.

127. Chiang PH, Wang L, Bonham CA, Liang X, Fung JJ, Lu L, and Qian S. Mechanistic insights into impaired dendritic cell function by rapamycin: inhibition of Jak2/Stat4 signaling pathway. J Immunol 172: 1355-1363, 2004.

128. Chobanian AV and Alexander RW. Exacerbation of atherosclerosis by hypertension. Potential mechanisms and clinical implications. Arch Intern Med 156: 1952-1956, 1996.

129. Christen S, Thomas SR, Garner B, and Stocker R. Inhibition by interferon-gamma of human mononuclear cell-mediated low density lipoprotein oxidation. Participation of tryptophan metabolism along the kynurenine pathway. J Clin Invest 93: 2149-2158, 1994.

130. Clinton SK, Underwood R, Hayes L, Sherman ML, Kufe DW, and Libby $\mathbf{P}$. Macrophage colony-stimulating factor gene expression in vascular cells and in experimental and human atherosclerosis. Am J Pathol 140: 301-316, 1992.

131. Cloez-Tayarani I, Petit-Bertron AF, Venters HD, and Cavaillon JM. Differential effect of serotonin on cytokine production in lipopolysaccharide-stimulated human peripheral blood mononuclear cells: involvement of 5-hydroxytryptamine2A receptors. Int Immunol 15: 233-240, 2003.

132. Cohen S. Cytokine: more than a new word, a new concept proposed by Stanley Cohen thirty years ago. Cytokine 28: 242-247, 2004.

133. Cohen S, Bigazzi PE, and Yoshida T. Commentary. Similarities of $\mathrm{T}$ cell function in cell-mediated immunity and antibody production. Cell Immunol 12: 150-159, 1974

134. Colotta F, Sciacca FL, Sironi M, Luini W, Rabiet MJ, and Mantovani A. Expression of monocyte chemotactic protein-1 by monocytes and endothelial cells exposed to thrombin. Am J Pathol 144: 975-985, 1994.

135. Combadiere C, Potteaux S, Gao JL, Esposito B, Casanova S, Lee EJ, Debre P, Tedgui A, Murphy PM, and Mallat Z. Decreased atherosclerotic lesion formation in CX3CR1/apolipoprotein E double knockout mice. Circulation 107: 1009-1016, 2003.
136. Compton SJ, Cairns JA, Holgate ST, and Walls AF. The role of mast cell tryptase in regulating endothelial cell proliferation, cytokine release, and adhesion molecule expression: tryptase induces expression of mRNA for IL-1 beta and IL-8 and stimulates the selective release of IL-8 from human umbilical vein endothelial cells. J Immunol 161: 1939-1946, 1998.

137. Constantinides P and Harkey M. Electron microscopic exploration of human endothelium in step-serial sections of early and advanced atherosclerotic lesions. Ann NY Acad Sci 598: 113-124, 1990.

138. Conway EM, Van de Wouwer M, Pollefeyt S, Jurk K, Van Aken H, De Vriese A, Weitz JI, Weiler H, Hellings PW, Schaeffer P, Herbert JM, Collen D, and Theilmeier G. The lectin-like domain of thrombomodulin confers protection from neutrophil-mediated tissue damage by suppressing adhesion molecule expression via nuclear factor kappaB and mitogen-activated protein kinase pathways. J Exp Med 196: 565-577, 2002.

139. Cooper JT, Stroka DM, Brostjan C, Palmetshofer A, Bach FH, and Ferran C. A20 blocks endothelial cell activation through a NF- $\kappa$ B-dependent mechanism. J Biol Chem 271: 18068-18073, 1996.

140. Coppinger JA, Cagney G, Toomey S, Kislinger T, Belton O, McRedmond JP, Cahill DJ, Emili A, Fitzgerald DJ, and Maguire PB. Characterization of the proteins released from activated platelets leads to localization of novel platelet proteins in human atherosclerotic lesions. Blood 103: 2096-2104, 2004.

141. Cornicelli JA, Butteiger D, Rateri DL, Welch K, and Daugherty A. Interleukin-4 augments acetylated LDL-induced cholesterol esterification in macrophages. J Lipid Res 41: 376-383, 2000.

142. Corti R, Fuster V, Fayad ZA, Worthley SG, Helft G, Chaplin WF, Muntwyler J, Viles-Gonzalez JF, Weinberger J, Smith DA, Mizsei G, and Badimon JJ. Effects of aggressive versus conventional lipid-lowering therapy by simvastatin on human atherosclerotic lesions: a prospective, randomized, double-blind trial with high-resolution magnetic resonance imaging. $\mathrm{J} \mathrm{Am} \mathrm{Coll} \mathrm{Car-}$ diol 46: 106-112, 2005.

143. Corti R, Fuster V, Fayad ZA, Worthley SG, Helft G, Smith D, Weinberger J, Wentzel J, Mizsei G, Mercuri M, and Badimon JJ. Lipid lowering by simvastatin induces regression of human atherosclerotic lesions: two years' follow-up by high-resolution noninvasive magnetic resonance imaging. Circulation 106: $2884-$ 2887, 2002.

144. Costanzo A, Moretti F, Burgio VL, Bravi C, Guido F, Levrero M, and Puri PL. Endothelial activation by angiotensin II through NFkappaB and p38 pathways: involvement of NFkappaB-inducible kinase (NIK), free oxygen radicals, and selective inhibition by aspirin. J Cell Physiol 195: 402-410, 2003.

145. Cottrez F, Hurst SD, Coffman RL, and Groux H. T regulatory cells 1 inhibit a Th2-specific response in vivo. J Immunol 165: 4848-4853, 2000.

146. Cui MZ, Penn MS, and Chisolm GM. Native and oxidized low density lipoprotein induction of tissue factor gene expression in smooth muscle cells is mediated by both Egr-1 and Sp1. J Biol Chem 274: 32795-32802, 1999.

147. Cummings CJ, Martin TR, Frevert CW, Quan JM, Wong VA, Mongovin SM, Hagen TR, Steinberg KP, and Goodman RB. Expression and function of the chemokine receptors CXCR1 and CXCR2 in sepsis. J Immunol 162: 2341-2346, 1999.

148. Cunningham KS and Gotlieb AI. The role of shear stress in the pathogenesis of atherosclerosis. Lab Invest 85: 9-23, 2005.

149. Curci JA and Thompson RW. Adaptive cellular immunity in aortic aneurysms: cause, consequence, or context? J Clin Invest 114: 168-171, 2004.

150. Cybulsky MI and Gimbrone MA Jr. Endothelial expression of a mononuclear leukocyte adhesion molecule during atherogenesis. Science 251: 788-791, 1991.

151. Cybulsky MI, Iiyama K, Li H, Zhu S, Chen M, Iiyama M, Davis V, Gutierrez-Ramos JC, Connelly PW, and Milstone DS. A major role for VCAM-1, but not ICAM-1, in early atherosclerosis. J Clin Invest 107: 1255-1262, 2001.

152. Damas JK, Waehre T, Yndestad A, Otterdal K, Hognestad A, Solum NO, Gullestad L, Froland SS, and Aukrust P. Interleu- 
kin-7-mediated inflammation in unstable angina: possible role of chemokines and platelets. Circulation 107: 2670-2676, 2003.

153. Dansky HM, Charlton SA, Harper MM, and Smith JD. T and B lymphocytes play a minor role in atherosclerotic plaque formation in the apolipoprotein E-deficient mouse. Proc Natl Acad Sci USA 94: 4642-4646, 1997.

154. Daugherty A, Pure E, Delfel-Butteiger D, Chen S, Leferovich J, Roselaar SE, and Rader DJ. The effects of total lymphocyte deficiency on the extent of atherosclerosis in apolipoprotein E-/mice. J Clin Invest 100: 1575-1580, 1997.

155. Daugherty A, Schonfeld G, Sobel BE, and Lange LG. Metabolism of very low density lipoproteins after cessation of cholesterol feeding in rabbits. A factor potentially contributing to the slow regression of atheromatous plaques. J Clin Invest 77: 1108-1115, 1986.

156. Davenport $\mathbf{P}$ and Tipping PG. The role of interleukin- 4 and interleukin-12 in the progression of atherosclerosis in apolipoprotein E-deficient mice. Am J Pathol 163: 1117-1125, 2003.

157. Davies MJ. A macro and micro view of coronary vascular insult in ischemic heart disease. Circulation 82: II38-46, 1990.

158. Davies MJ. Stability and instability: two faces of coronary atherosclerosis-The Paul Dudley White Lecture 1995. Circulation 94: 2013-2020, 1996.

159. Davies MJ and Thomas T. The pathological basis and microanatomy of occlusive thrombus formation in human coronary arteries. Philos Trans R Soc Lond B Biol Sci 294: 225-229, 1981.

160. Davies PF. Flow-mediated endothelial mechanotransduction. Physiol Rev 75: 519-560, 1995.

161. Dawson TC, Kuziel WA, Osahar TA, and Maeda N. Absence of CC chemokine receptor-2 reduces atherosclerosis in apolipoprotein E-deficient mice. Atherosclerosis 143: 205-211, 1999.

162. De Caterina R and Zampolli A. From asthma to atherosclerosis5-lipoxygenase, leukotrienes, and inflammation. N Engl J Med 350: 4-7, 2004.

163. Dechend R, Viedt C, Muller DN, Ugele B, Brandes RP, Wallukat G, Park JK, Janke J, Barta P, Theuer J, Fiebeler A, Homuth V, Dietz R, Haller H, Kreuzer J, and Luft FC. AT1 receptor agonistic antibodies from preeclamptic patients stimulate NADPH oxidase. Circulation 107: 1632-1639, 2003.

164. DeForge LE, Kenney JS, Jones ML, Warren JS, and Remick DG. Biphasic production of IL-8 in lipopolysaccharide (LPS)-stimulated human whole blood. Separation of LPS- and cytokine-stimulated components using anti-tumor necrosis factor and anti-IL-1 antibodies. J Immunol 148: 2133-2141, 1992.

165. Degryse B and de Virgilio M. The nuclear protein HMGB1, a new kind of chemokine? FEBS Lett 553: 11-17, 2003.

166. De Martin R, Hoeth M, Hofer-Warbinek R, and Schmid JA. The transcription factor NF- $\kappa \mathrm{B}$ and the regulation of vascular cell function. Arterioscler Thromb Vasc Biol 20: E83-E88, 2000.

167. De Nooijer R, von der Thusen JH, Verkleij CJ, Kuiper J, Jukema JW, van der Wall EE, van Berkel JC, and Biessen EA. Overexpression of IL-18 decreases intimal collagen content and promotes a vulnerable plaque phenotype in apolipoprotein-E-deficient mice. Arterioscler Thromb Vasc Biol 24: 2313-2319, 2004.

168. Descoteaux A and Matlashewski G. Regulation of tumor necrosis factor gene expression and protein synthesis in murine macrophages treated with recombinant tumor necrosis factor. $J$ Immunol 145: 846-853, 1990.

169. Devlin CM, Kuriakose G, Hirsch E, and Tabas I. Genetic alterations of IL-1 receptor antagonist in mice affect plasma cholesterol level and foam cell lesion size. Proc Natl Acad Sci USA 99: 62806285, 2002.

170. De Waal Malefyt R, Yssel H, and de Vries JE. Direct effects of IL-10 on subsets of human CD4 + T cell clones and resting T cells. Specific inhibition of IL-2 production and proliferation. J Immunol 150: 4754-4765, 1993.

171. Dewberry R, Holden H, Crossman D, and Francis S. Interleukin-1 receptor antagonist expression in human endothelial cells and atherosclerosis. Arterioscler Thromb Vasc Biol 20: 2394-2400, 2000 .

172. Dhaunsi GS, Kaur J, and Turner RB. Role of NADPH oxidase in cytomegalovirus-induced proliferation of human coronary artery smooth muscle cells. J Biomed Sci 10: 505-509, 2003.
173. Dhore CR, Cleutjens JP, Lutgens E, Cleutjens KB, Geusens PP, Kitslaar PJ, Tordoir JH, Spronk HM, Vermeer C, and Daemen MJ. Differential expression of bone matrix regulatory proteins in human atherosclerotic plaques. Arterioscler Thromb Vasc Biol 21: 1998-2003, 2001.

174. Dieckmann D, Bruett CH, Ploettner H, Lutz MB, and Schuler G. Human CD4 $(+) \mathrm{CD} 25(+)$ regulatory, contact-dependent T cells induce interleukin 10-producing, contact-independent type 1-like regulatory T cells. J Exp Med 196: 247-253, 2002.

175. Dinarello CA, Ikejima T, Warner SJ, Orencole SF, Lonnemann G, Cannon JG, and Libby P. Interleukin 1 induces interleukin 1. I. Induction of circulating interleukin 1 in rabbits in vivo and in human mononuclear cells in vitro. J Immunol 139: 19021910, 1987.

176. Dinarello CA, Novick D, Rubinstein M, and Lonnemann G. Interleukin 18 and interleukin 18 binding protein: possible role in immunosuppression of chronic renal failure. Blood Purif 21: 258270, 2003

177. Distler JH, Jungel A, Huber LC, Seemayer CA, Reich CF, 3rd Gay RE, Michel BA, Fontana A, Gay S, Pisetsky DS, and Distler $\mathbf{O}$. The induction of matrix metalloproteinase and cytokine expression in synovial fibroblasts stimulated with immune cell microparticles. Proc Natl Acad Sci USA 102: 2892-2897, 2005.

178. Dol F, Martin G, Staels B, Mares AM, Cazaubon C, Nisato D, Bidouard JP, Janiak P, Schaeffer P, and Herbert JM. Angiotensin AT1 receptor antagonist irbesartan decreases lesion size, chemokine expression, and macrophage accumulation in apolipoprotein E-deficient mice. J Cardiovasc Pharmacol 38: 395-405, 2001.

179. Dong C, Yang DD, Tournier C, Whitmarsh AJ, Xu J, Davis RJ, and Flavell RA. JNK is required for effector T-cell function but not for T-cell activation. Nature 405: 91-94, 2000.

180. Dorffel Y, Latsch C, Stuhlmuller B, Schreiber S, Scholze S, Burmester GR, and Scholze J. Preactivated peripheral blood monocytes in patients with essential hypertension. Hypertension 34: 113-117, 1999.

181. Dumonde DC, Wolstencroft RA, Panayi GS, Matthew M, Morley J, and Howson WT. "Lymphokines": non-antibody mediators of cellular immunity generated by lymphocyte activation. Nature 224: 38-42, 1969.

182. Durand E, Scoazec A, Lafont A, Boddaert J, Al Hajzen A, Addad F, Mirshahi M, Desnos M, Tedgui A, and Mallat Z. In vivo induction of endothelial apoptosis leads to vessel thrombosis and endothelial denudation: a clue to the understanding of the mechanisms of thrombotic plaque erosion. Circulation 109: 25032506, 2004

183. Durk T, Panther E, Muller T, Sorichter S, Ferrari D, Pizzirani C, Di Virgilio F, Myrtek D, Norgauer J, and Idzko M. 5-Hydroxytryptamine modulates cytokine and chemokine production in LPS-primed human monocytes via stimulation of different 5-HTR subtypes. Int Immunol 17: 599-606, 2005.

184. Dwyer JH, Allayee H, Dwyer KM, Fan J, Wu H, Mar R, Lusis AJ, and Mehrabian M. Arachidonate 5-lipoxygenase promoter genotype, dietary arachidonic acid, and atherosclerosis. $N$ Engl J Med 350: 29-37, 2004

185. Edens HA and Parkos CA. Modulation of epithelial and endothelial paracellular permeability by leukocytes. Adv Drug Deliv Rev 41: 315-328, 2000.

186. Edfeldt K, Swedenborg J, Hansson GK, and Yan ZQ. Expression of toll-like receptors in human atherosclerotic lesions: a possible pathway for plaque activation. Circulation 105: 1158-1161, 2002.

187. Elhage R, Clamens S, Besnard S, Mallat Z, Tedgui A, Arnal J, Maret A, and Bayard F. Involvement of interleukin-6 in atherosclerosis but not in the prevention of fatty streak formation by 17beta-estradiol in apolipoprotein E-deficient mice. Atherosclerosis 156: 315-320, 2001.

188. Elhage R, Jawien J, Rudling M, Ljunggren HG, Takeda K, Akira S, Bayard F, and Hansson GK. Reduced atherosclerosis in interleukin-18 deficient apolipoprotein E-knockout mice. Cardiovasc Res 59: 234-240, 2003.

189. Elhage R, Maret A, Pieraggi MT, Thiers JC, Arnal JF, and Bayard F. Differential effects of interleukin-1 receptor antagonist 
and tumor necrosis factor binding protein on fatty-streak formation in apolipoprotein E-deficient mice. Circulation 97: 242-244, 1998.

190. Elloso MM, Azrolan N, Sehgal SN, Hsu PL, Phiel KL, Kopec CA, Basso MD, and Adelman SJ. Protective effect of the immunosuppressant sirolimus against aortic atherosclerosis in apo E-deficient mice. Am J Transplant 3: 562-569, 2003.

191. Engelse MA, Neele JM, van Achterberg TA, van Aken BE, van Schaik RH, Pannekoek H, and de Vries CJ. Human activin-A is expressed in the atherosclerotic lesion and promotes the contractile phenotype of smooth muscle cells. Circ Res 85: 931-939, 1999.

192. Epstein SE, Stabile E, Kinnaird T, Lee CW, Clavijo L, and Burnett MS. Janus phenomenon: the interrelated tradeoffs inherent in therapies designed to enhance collateral formation and those designed to inhibit atherogenesis. Circulation 109: 2826-2831, 2004.

193. Esmon CT. The impact of the inflammatory response on coagulation. Thromb Res 114: 321-327, 2004.

194. Esposito C, Gerlag H, Brett J, Stern D, and Vlassara H. Endothelial receptor-mediated binding of glucose-modified albumin is associated with increased monolayer permeability and modulation of cell surface coagulant properties. J Exp Med 170: 1387-1407, 1989.

195. Esteban V, Ruperez M, Sanchez-Lopez E, Rodriguez-Vita J, Lorenzo O, Demaegdt H, Vanderheyden P, Egido J, and RuizOrtega M. Angiotensin IV activates the nuclear transcription factor-kappaB and related proinflammatory genes in vascular smooth muscle cells. Circ Res 96: 965-973, 2005.

196. Fabricant CG, Fabricant J, Litrenta MM, and Minick CR. Virus-induced atherosclerosis. J Exp Med 148: 335-340, 1978.

197. Faggiotto A, Ross R, and Harker L. Studies of hypercholesterolemia in the non human primate. I. Changes that lead to fatty streak formation. Arteriosclerosis 4: 323-340, 1984.

198. Falk E. Plaque rupture with severe pre-existing stenosis precipitating coronary thrombosis. Characteristics of coronary atherosclerotic plaques underlying fatal occlusive thrombi. Br Heart $J 50$ : 127-134, 1983.

199. Farb A, Burke AP, Tang AL, Liang YH, Mannan P, Smialek J, and Virmani R. Coronary plaque erosion without rupture into a lipid core: a frequent cause of coronary thrombosis in sudden coronary death. Circulation 93: 1354-1363, 1996.

200. Feinberg MW, Shimizu K, Lebedeva M, Haspel R, Takayama K, Chen Z, Frederick JP, Wang XF, Simon DI, Libby P, Mitchell RN, and Jain MK. Essential role for Smad3 in regulating MCP-1 expression and vascular inflammation. Circ Res 94: 601-608, 2004.

201. Feinberg MW, Watanabe M, Lebedeva MA, Depina AS, Hanai J, Mammoto T, Frederick JP, Wang XF, Sukhatme VP, and Jain MK. Transforming growth factor-beta1 inhibition of vascular smooth muscle cell activation is mediated via Smad3. J Biol Chem 279: 16388-16393, 2004.

202. Ferns GAA, Reidy MA, and Ross R. Balloon catheter de-endothelialization of the nude rat carotid: response to injury in the absence of functional lymphocyte-T. Am J Pathol 138: 1045-1057, 1991.

203. Fichtlscherer S, Breuer S, Heeschen C, Dimmeler S, and Zeiher AM. Interleukin-10 serum levels and systemic endothelial vasoreactivity in patients with coronary artery disease. $J$ Am Coll Cardiol 44: 44-49, 2004.

204. Folcik VA, Aamir R, and Cathcart MK. Cytokine modulation of LDL oxidation by activated human monocytes. Arterioscler Thromb Vasc Biol 17: 1954-1961, 1997.

205. Fong AM, Robinson LA, Steeber DA, Tedder TF, Yoshie O, Imai T, and Patel DD. Fractalkine and CX3CR1 mediate a novel mechanism of leukocyte capture, firm adhesion, and activation under physiologic flow. J Exp Med 188: 1413-1419, 1998.

206. Fong LG, Albert TSE, and Hom SE. Inhibition of the macrophage-induced oxidation of low density lipoprotein by interferongamma. J Lipid Res 35: 893-904, 1994.

207. Fontenot JD and Rudensky AY. A well adapted regulatory contrivance: regulatory $\mathrm{T}$ cell development and the forkhead family transcription factor Foxp3. Nat Immunol 6: 331-337, 2005.

208. Forster BA, Javed Q, Leake DS, and Weinberg PD. Highresolution mapping of the frequency of lipid deposits in thoracic aortae from cholesterol-fed and heritable hyperlipidaemic rabbits. Atherosclerosis 120: 249-253, 1996.

209. Fourcade O, Simon MF, Viode C, Rugani FL, Ragab A, Fournie B, Sarda L, and Chap H. Secretory phospholipase $\mathrm{A}_{2}$ generates the novel lipid mediator lysophosphatidic acid in membrane microvesicles shed from activated cells. Cell 80: 919-927, 1995.

210. Fredrikson GN, Hedblad B, Berglund G, Alm R, Ares M, Cercek B, Chyu KY, Shah PK, and Nilsson J. Identification of immune responses against aldehyde-modified peptide sequences in apoB associated with cardiovascular disease. Arterioscler Thromb Vasc Biol 23: 872-878, 2003.

211. Freeman CM, Chiu BC, Stolberg VR, Hu J, Zeibecoglou K, Lukacs NW, Lira SA, Kunkel SL, and Chensue SW. CCR8 is expressed by antigen-elicited, IL-10-producing CD4+CD25+ T cells, which regulate Th2-mediated granuloma formation in mice. J Immunol 174: 1962-1970, 2005.

212. Freigang S, Horkko S, Miller E, Witztum JL, and Palinski W. Immunization of LDL receptor-deficient mice with homologous malondialdehyde-modified and native LDL reduces progression of atherosclerosis by mechanisms other than induction of high titers of antibodies to oxidative neoepitopes. Arterioscler Thromb Vasc Biol 18: 1972-1982, 1998.

213. Frostegard J, Huang YH, Ronnelid J, and Schafer-Elinder L. Platelet-activating factor and oxidized LDL induce immune activation by a common mechanism. Arterioscler Thromb Vasc Biol 17: 963-968, 1997.

214. Frostegard J, Ulfgren AK, Nyberg P, Hedin U, Swedenborg J, Andersson U, and Hansson GK. Cytokine expression in advanced human atherosclerotic plaques: dominance of pro-inflammatory (Th1) and macrophage-stimulating cytokines. Atherosclerosis 145: 33-43, 1999.

215. Frostegard J, Wu R, Giscombe R, Holm G, Lefvert AK, and Nilsson J. Induction of T-cell activation by oxidized low density lipoprotein. Arterioscler Thromb 12: 461-467, 1992.

216. Fukudome K and Esmon CT. Identification, cloning, and regulation of a novel endothelial cell protein C/activated protein c receptor. J Biol Chem 269: 26486-26491, 1994.

217. Funakoshi Y, Ichiki T, Shimokawa H, Egashira K, Takeda K, Kaibuchi K, Takeya M, Yoshimura T, and Takeshita A. Rhokinase mediates angiotensin II-induced monocyte chemoattractant protein-1 expression in rat vascular smooth muscle cells. Hypertension 38: 100-104, 2001.

218. Fuster V, Fayad ZA, and Badimon JJ. Acute coronary syndromes: biology. Lancet 353 Suppl 2: SII5-9, 1999.

219. Galea P, Thibault G, Lacord M, Bardos P, and Lebranchu Y. $\mathrm{IL}-4$, but not tumor necrosis factor- $\alpha$, increases endothelial cell adhesiveness for lymphocytes by activating a cAMP-dependent pathway. J Immunol 151: 588-596, 1993.

220. Galis ZS, Muszynski M, Sukhova GK, Simonmorrissey E, Unemori EN, Lark MW, Amento E, and Libby P. Cytokinestimulated human vascular smooth muscle cells synthesize a complement of enzymes required for extracellular matrix digestion. Circ Res 75: 181-189, 1994

221. Gao Y, Herndon JM, Zhang H, Griffith TS, and Ferguson TA. Anti-inflammatory effects of CD95 ligand (FasL)-induced apoptosis. J Exp Med 188: 887-896, 1998.

222. Geng YJ and Libby P. Progression of atheroma: a struggle between death and procreation. Arterioscler Thromb Vasc Biol 22: 1370-1380, 2002

223. Geng YJ, Wu Q, Muszynski M, Hansson GK, and Libby $\mathbf{P}$. Apoptosis of vascular smooth muscle cells induced by in vitro stimulation with interferon-gamma, tumor necrosis factor-alpha, and interleukin-1 beta. Arterioscler Thromb Vasc Biol 16: 19-27, 1996.

224. George J, Afek A, Gilburd B, Blank M, Levy Y, Aron-Maor A, Levkovitz H, Shaish A, Goldberg I, Kopolovic J, Harats D, and Shoenfeld Y. Induction of early atherosclerosis in LDL-receptordeficient mice immunized with beta2-glycoprotein I. Circulation 98: 1108-1115, 1998.

225. George J, Mulkins M, Shaish A, Casey S, Schatzman R, Sigal E, and Harats D. Interleukin (IL)-4 deficiency does not influence fatty streak formation in C57BL/6 mice. Atherosclerosis 153: 403411, 2000 . 
226. George J, Shoenfeld Y, Afek A, Gilburd B, Keren P, Shaish A, Kopolovic J, Wick G, and Harats D. Enhanced fatty streak formation in C57BL/6J mice by immunization with heat shock protein-65. Arterioscler Thromb Vasc Biol 19: 505-510, 1999.

227. George J, Shoenfeld Y, Gilburd B, Afek A, Shaish A, and Harats D. Requisite role for interleukin- 4 in the acceleration of fatty streaks induced by heat shock protein 65 or Mycobacterium tuberculosis. Circ Res 86: 1203-1210, 2000.

228. Gerdes N, Sukhova GK, Libby P, Reynolds RS, Young JL, and Schonbeck U. Expression of interleukin (IL)-18 and functional IL-18 receptor on human vascular endothelial cells, smooth muscle cells, and macrophages: implications for atherogenesis. J Exp Med 195: 245-257, 2002.

229. Gerrity RG. The role of the monocyte in atherogenesis. II. Migration of foam cells from atherosclerotic lesions. Am J Pathol 103: 191-200, 1981.

230. Giachelli CM, Bae N, Almeida M, Denhardt DT, Alpers CE, and Schwartz SM. Osteopontin is elevated during neointima formation in rat arteries and is a novel component of human atherosclerotic plaques. J Clin Invest 92: 1686-1696, 1993.

231. Giesen PL, Rauch U, Bohrmann B, Kling D, Roque M, Fallon JT, Badimon JJ, Himber J, Riederer MA, and Nemerson Y. Blood-borne tissue factor: another view of thrombosis. Proc Natl Acad Sci USA 96: 2311-2315, 1999.

232. Gimbrone MA Jr and Buchanan MR. Interactions of platelets and leukocytes with vascular endothelium: in vitro studies. Ann NY Acad Sci 401: 171-183, 1982.

233. Gimbrone MA Jr, Obin MS, Brock AF, Luis EA, Hass PE, Hebert CA, Yip YK, Leung DW, Lowe DG, and Kohr WJ. Endothelial interleukin-8: a novel inhibitor of leukocyte-endothelial interactions. Science 246: 1601-1603, 1989.

234. Glagov S, Weisenberg E, Zarins CK, Stankunavicius R, and Kolettis GJ. Compensatory enlargement of human atherosclerotic coronary arteries. N Engl J Med 316: 1371-1375, 1987.

235. Glass CK and Witztum JL. Atherosclerosis the road ahead. Cell 104: 503-516, 2001

236. Gojova A, Brun V, Esposito B, Cottrez F, Gourdy P, Ardouin $\mathbf{P}$, Tedgui A, Mallat $\mathbf{Z}$, and Groux $\mathbf{H}$. Specific abrogation of transforming growth factor- $\beta$ signaling in $\mathrm{T}$ cells alters atherosclerotic lesion size and composition in mice. Blood 102: 4052-4058, 2003.

237. Goldstein JL, Ho YK, Basu SK, and Brown MS. Binding site on macrophages that mediates uptake and degradation of acetylated low density lipoprotein, producing massive cholesterol deposition. Proc Natl Acad Sci USA 76: 333-337, 1979.

238. Golledge J, McCann M, Mangan S, Lam A, and Karan M. Osteoprotegerin and osteopontin are expressed at high concentrations within symptomatic carotid atherosclerosis. Stroke 35: 16361641, 2004.

239. Goodarzi K, Goodarzi M, Tager AM, Luster AD, and von Andrian UH. Leukotriene B4 and BLT1 control cytotoxic effector T cell recruitment to inflamed tissues. Nat Immunol 4: 965-973, 2003.

240. Gordon JR and Galli SJ. Mast cells as a source of both preformed and immunologically inducible TNF-alpha/cachectin. Nature 346: 274-276, 1990.

241. Gosling J, Slaymaker S, Gu L, Tseng S, Zlot CH, Young SG, Rollins BJ, and Charo IF. MCP-1 deficiency reduces susceptibility to atherosclerosis in mice that overexpress human apolipoprotein B. J Clin Invest 103: 773-778, 1999.

242. Grafe M, Auch-Schwelk W, Zakrzewicz A, Regitz-Zagrosek V, Bartsch P, Graf K, Loebe M, Gaehtgens P, and Fleck E. Angiotensin II-induced leukocyte adhesion on human coronary endothelial cells is mediated by E-selectin. Circ Res 81: 804-811, 1997.

243. Grainger DJ. Transforming growth factor beta and atherosclerosis: so far, so good for the protective cytokine hypothesis. Arterioscler Thromb Vasc Biol 24: 399-404, 2004

244. Grainger DJ, Kemp BR, Metcalfe JC, Liu AC, Lawn RM, Williams NR, Grace AA, Schofield PM, and Chauhan A. The serum concentration of active transforming growth factor-beta is severely depressed in advanced atherosclerosis. Nature Med 1: 74-79, 1995.

245. Grainger DJ, Mosedale DE, Metcalfe JC, and Bottinger EP. Dietary fat and reduced levels of TGF $\beta 1$ act synergistically to promote activation of the vascular endothelium and formation of lipid lesions. J Cell Sci 113: 2355-2361, 2000.

246. Grainger DJ, Reckless J, and McKilligin E. Apolipoprotein E modulates clearance of apoptotic bodies in vitro and in vivo, resulting in a systemic proinflammatory state in apolipoprotein E-deficient mice. J Immunol 173: 6366-6375, 2004.

247. Grandaliano G, Valente AJ, and Abboud HE. A novel biologic activity of thrombin: stimulation of monocyte chemotactic protein production. J Exp Med 179: 1737-1741, 1994.

248. Gravallese EM. Osteopontin: a bridge between bone and the immune system. J Clin Invest 112: 147-149, 2003.

249. Gravel SP and Servant MJ. Roles of an IkappaB kinase-related pathway in human cytomegalovirus-infected vascular smooth muscle cells: a molecular link in pathogen-induced proatherosclerotic conditions. J Biol Chem 280: 7477-7486, 2005.

250. Grayston JT, Kronmal RA, Jackson LA, Parisi AF, Muhlestein JB, Cohen JD, Rogers WJ, Crouse JR, Borrowdale SL, Schron $\mathbf{E}$, and Knirsch C. Azithromycin for the secondary prevention of coronary events. $N$ Engl J Med 352: 1637-1645, 2005.

251. Green EA, Gorelik L, McGregor CM, Tran EH, and Flavell RA. $\mathrm{CD} 4+\mathrm{CD} 25+\mathrm{T}$ regulatory cells control anti-islet CD8 $+\mathrm{T}$ cells through TGF-beta-TGF-beta receptor interactions in type 1 diabetes. Proc Natl Acad Sci USA 100: 10878-10883, 2003.

252. Griendling KK and FitzGerald GA. Oxidative stress and cardiovascular injury. Part I: basic mechanisms and in vivo monitoring of ROS. Circulation 108: 1912-1916, 2003.

253. Griendling KK, Minieri CA, Ollerenshaw JD, and Alexander RW. Angiotensin II stimulates NADH and NADPH oxidase activity in cultured vascular smooth muscle cells. Circ Res 74: 1141-1148, 1994.

254. Groux H, Fournier N, and Cottrez F. Role of dendritic cells in the generation of regulatory T cells. Semin Immunol 16: 99-106, 2004.

255. Groux H, O'Garra A, Bigler M, Rouleau M, Antonenko S, de Vries JE, and Roncarolo MG. A CD4+ T-cell subset inhibits antigen-specific T-cell responses and prevents colitis. Nature 389: 737-742, 1997.

256. Grundy SM, Cleeman JI, Merz CN, Brewer HB Jr, Clark LT, Hunninghake DB, Pasternak RC, Smith SC Jr, and Stone NJ. Implications of recent clinical trials for the National Cholesterol Education Program Adult Treatment Panel III guidelines. Circulation 110: 227-239, 2004

257. Grunig G, Corry DB, Leach MW, Seymour BW, Kurup VP, and Rennick DM. Interleukin-10 is a natural suppressor of cytokine production and inflammation in a murine model of allergic bronchopulmonary aspergillosis. J Exp Med 185: 1089-1099, 1997.

258. Gu L, Okada Y, Clinton SK, Gerard C, Sukhova GK, Libby P, and Rollins BJ. Absence of monocyte chemoattractant protein-1 reduces atherosclerosis in low density lipoprotein receptor-deficient mice. Mol Cell 2: 275-281, 1998.

259. Gupta S, Barrett T, Whitmarsh AJ, Cavanagh J, Sluss HK, Derijard B, and Davis RJ. Selective interaction of JNK protein kinase isoforms with transcription factors. EMBO J 15: 2760-2770, 1996.

260. Gupta S, Pablo AM, Jiang XC, Wang N, Tall AR, and Schindler C. IFN-gamma potentiates atherosclerosis in apoE knock-out mice. J Clin Invest 99: 2752-2761, 1997.

261. Hagenbaugh A, Sharma S, Dubinett SM, Wei SHY, Aranda R, Cheroutre H, Fowell DJ, Binder S, Tsao B, Locksley RM, Moore KW, and Kronenberg $\mathbf{M}$. Altered immune responses in interleukin 10 transgenic mice. J Exp Med 185: 2101-2110, 1997.

262. Hajra L, Evans AI, Chen M, Hyduk SJ, Collins T, and Cybulsky MI. The NF- $\kappa$ B signal transduction pathway in aortic endothelial cells is primed for activation in regions predisposed to atherosclerotic lesion formation. Proc Natl Acad Sci USA 97: 9052-9057, 2000.

263. Han Y, Runge MS, and Brasier AR. Angiotensin II induces interleukin-6 transcription in vascular smooth muscle cells through pleiotropic activation of nuclear factor-kappa B transcription factors. Circ Res 84: 695-703, 1999.

264. Hansson GK. Immune mechanisms in atherosclerosis. Arterioscler Thromb Vasc Biol 21: 1876-1890, 2001. 
265. Hansson GK. Inflammation, atherosclerosis, and coronary artery disease. $N$ Engl J Med 352: 1685-1695, 2005.

266. Hansson GK, Holm J, Holm S, Fotev Z, Hedrich HJ, and Fingerle J. Lymphocytes-t inhibit the vascular response to injury. Proc Natl Acad Sci USA 88: 10530-10534, 1991.

267. Hansson GK, Holm J, and Jonasson L. Detection of activated T lymphocytes in the human atherosclerotic plaque. Am J Pathol 135: $169-175,1989$.

268. Hansson GK, Libby P, Schonbeck U, and Yan ZQ. Innate and adaptive immunity in the pathogenesis of atherosclerosis. Circ Res 91: 281-291, 2002.

269. Harats D, Yacov N, Gilburd B, Shoenfeld Y, and George J. Oral tolerance with heat shock protein 65 attenuates Mycobacterium tuberculosis-induced and high-fat-diet-driven atherosclerotic lesions. J Am Coll Cardiol 40: 1333-1338, 2002.

270. Harris TB, Ferrucci L, Tracy RP, Corti MC, Wacholder S, Ettinger WH Jr, Heimovitz H, Cohen HJ, and Wallace R. Associations of elevated interleukin- 6 and C-reactive protein levels with mortality in the elderly. Am J Med 106: 506-512, 1999.

271. Hasty AH, Shimano H, Osuga J, Namatame I, Takahashi A, Yahagi N, Perrey S, lizuka Y, Tamura Y, Amemiya-Kudo M, Yoshikawa T, Okazaki H, Ohashi K, Harada K, Matsuzaka T, Sone H, Gotoda T, Nagai R, Ishibashi S, and Yamada N. Severe hypercholesterolemia, hypertriglyceridemia, and atherosclerosis in mice lacking both leptin and the low density lipoprotein receptor. J Biol Chem 276: 37402-37408, 2001.

272. Hattori K, Heissig B, Tashiro K, Honjo T, Tateno M, Shieh JH, Hackett NR, Quitoriano MS, Crystal RG, Rafii S, and Moore MA. Plasma elevation of stromal cell-derived factor-1 induces mobilization of mature and immature hematopoietic progenitor and stem cells. Blood 97: 3354-3360, 2001.

273. Hayakawa M, Miyashita H, Sakamoto I, Kitagawa M, Tanaka H, Yasuda H, Karin M, and Kikugawa K. Evidence that reactive oxygen species do not mediate NF-kappaB activation. EMBO J 22: 3356-3366, 2003.

274. Hayes IM, Jordan NJ, Towers S, Smith G, Paterson JR, Earnshaw JJ, Roach AG, Westwick J, and Williams RJ. Human vascular smooth muscle cells express receptors for CC chemokines. Arterioscler Thromb Vasc Biol 18: 397-403, 1998.

275. Hechtman DH, Cybulsky MI, Fuchs HJ, Baker JB, and Gimbrone MA Jr. Intravascular IL-8. Inhibitor of polymorphonuclear leukocyte accumulation at sites of acute inflammation. J Immunol 147: 883-892, 1991.

276. Heeschen C, Dimmeler S, Hamm CW, Fichtlscherer S, Boersma E, Simoons ML, and Zeiher AM. Serum level of the antiinflammatory cytokine interleukin-10 is an important prognostic determinant in patients with acute coronary syndromes. Circulation 107: 2109-2114, 2003.

277. Heeschen C, Dimmeler S, Hamm CW, van den Brand MJ, Boersma E, Zeiher AM, and Simoons ML. Soluble CD40 ligand in acute coronary syndromes. $N$ Engl $J$ Med 348: 1104-1111, 2003.

278. Heissig B, Hattori K, Dias S, Friedrich M, Ferris B, Hackett NR, Crystal RG, Besmer P, Lyden D, Moore MA, Werb Z, and Rafii S. Recruitment of stem and progenitor cells from the bone marrow niche requires MMP-9 mediated release of kit-ligand. Cell 109: 625-637, 2002.

279. Helgadottir A, Manolescu A, Thorleifsson G, Gretarsdottir S, Jonsdottir H, Thorsteinsdottir U, Samani NJ, Gudmundsson G, Grant SF, Thorgeirsson G, Sveinbjornsdottir S, Valdimarsson EM, Matthiasson SE, Johannsson H, Gudmundsdottir O, Gurney ME, Sainz J, Thorhallsdottir M, Andresdottir M, Frigge ML, Topol EJ, Kong A, Gudnason V, Hakonarson H, Gulcher JR, and Stefansson K. The gene encoding 5-lipoxygenase activating protein confers risk of myocardial infarction and stroke. Nat Genet 36: 233-239, 2004.

280. Heller EA, Liu E, Tager AM, Sinha S, Roberts JD, Koehn SL, Libby P, Aikawa ER, Chen JQ, Huang P, Freeman MW, Moore KJ, Luster AD, and Gerszten RE. Inhibition of atherogenesis in BLT1-deficient mice reveals a role for LTB4 and BLT1 in smooth muscle cell recruitment. Circulation 112: 578-586, 2005.

281. Henn V, Slupsky JR, Gräfe M, Anagnostopoulos I, Förster R, Müller-Berghaus G, and Kroczek RA. CD40 ligand on activated platelets triggers an inflammatory reaction of endothelial cells. Nature 391: 591-594, 1998.

282. Henn V, Steinbach S, Buchner K, Presek P, and Kroczek RA. The inflammatory action of CD40 ligand (CD154) expressed on activated human platelets is temporally limited by coexpressed CD40. Blood 98: 1047-1054, 2001.

283. Hernandez-Presa M, Bustos C, Ortego M, Tunon J, Renedo G, Ruiz-Ortega M, and Egido J. Angiotensin-converting enzyme inhibition prevents arterial nuclear factor-kappa B activation, monocyte chemoattractant protein-1 expression, and macrophage infiltration in a rabbit model of early accelerated atherosclerosis. Circulation 95: 1532-1541, 1997.

284. Hernandez-Presa MA, Bustos C, Ortego M, Tunon J, Ortega L, and Egido J. ACE inhibitor quinapril reduces the arterial expression of NF-kappaB-dependent proinflammatory factors but not of collagen I in a rabbit model of atherosclerosis. Am J Pathol 153: 1825-1837, 1998.

285. Hirschfield GM, Gallimore JR, Kahan MC, Hutchinson WL, Sabin CA, Benson GM, Dhillon AP, Tennent GA, and Pepys MB. Transgenic human C-reactive protein is not proatherogenic in apolipoprotein E-deficient mice. Proc Natl Acad Sci USA 102: 8309-8314, 2005

286. Hofmann MA, Drury S, Fu C, Qu W, Taguchi A, Lu Y, Avila C, Kambham N, Bierhaus A, Nawroth $P$, Neurath MF, Slattery T, Beach D, McClary J, Nagashima M, Morser J, Stern D, and Schmidt AM. RAGE mediates a novel proinflammatory axis: a central cell surface receptor for S100/calgranulin polypeptides. Cell 97: 889-901, 1999 .

287. Hollestelle SC, De Vries MR, Van Keulen JK, Schoneveld AH, Vink A, Strijder CF, Van Middelaar BJ, Pasterkamp G, Quax PH, and De Kleijn DP. Toll-like receptor 4 is involved in outward arterial remodeling. Circulation 109: 393-398, 2004.

288. Horkko S, Binder CJ, Shaw PX, Chang MK, Silverman G, Palinski W, and Witztum JL. Immunological responses to oxidized LDL. Free Radical Biol Med 28: 1771-1779, 2000.

289. Houtkamp MA, van Der Wal AC, de Boer OJ, van Der Loos CM, de Boer PA, Moorman AF, and Becker AE. Interleukin-15 expression in atherosclerotic plaques: an alternative pathway for T-cell activation in atherosclerosis? Arterioscler Thromb Vasc Biol 21: 1208-1213, 2001.

290. Hoyne GF, O'Hehir RE, Wraith DC, Thomas WR, and Lamb JR. Inhibition of $\mathrm{T}$ cell and antibody responses to house dust mite allergen by inhalation of the dominant $\mathrm{T}$ cell epitope in naive and sensitized mice. J Exp Med 178: 1783-1788, 1993.

291. Hsu HY, Nicholson AC, and Hajjar DP. Inhibition of macrophage scavenger receptor activity by tumor necrosis factor-alpha is transcriptionally and post-transcriptionally regulated. J Biol Chem 271: 7767-7773, 1996.

292. Hu H, Pierce GN, and Zhong G. The atherogenic effects of chlamydia are dependent on serum cholesterol and specific to Chlamydia pneumoniae. J Clin Invest 103: 747-753, 1999.

293. Huang FP, Platt N, Wykes M, Major JR, Powell TJ, Jenkins CD, and MacPherson GG. A discrete subpopulation of dendritic cells transports apoptotic intestinal epithelial cells to T cell areas of mesenteric lymph nodes. $J$ Exp Med 191: 435-444, 2000.

294. Huber SA, Sakkinen P, Conze D, Hardin N, and Tracy R. Interleukin-6 exacerbates early atherosclerosis in mice. Arterioscler Thromb Vasc Biol 19: 2364-2367, 1999.

295. Huber SA, Sakkinen P, David C, Newell MK, and Tracy RP. T helper-cell phenotype regulates atherosclerosis in mice under conditions of mild hypercholesterolemia. Circulation 103: 2610-2616, 2001.

296. Hugel B, Martinez MC, Kunzelmann C, and Freyssinet JM. Membrane microparticles: two sides of the coin. Physiology 20: 22-27, 2005.

297. Hull KM, Shoham N, Chae JJ, Aksentijevich I, and Kastner DL. The expanding spectrum of systemic autoinflammatory disorders and their rheumatic manifestations. Curr Opin Rheumatol 15: 61-69, 2003.

298. Huo Y, Schober A, Forlow SB, Smith DF, Hyman MC, Jung S, Littman DR, Weber C, and Ley K. Circulating activated platelets exacerbate atherosclerosis in mice deficient in apolipoprotein $\mathrm{E}$. Nat Med 9: 61-67, 2003. 
299. Huynh ML, Fadok VA, and Henson PM. Phosphatidylserinedependent ingestion of apoptotic cells promotes TGF-beta1 secretion and the resolution of inflammation. J Clin Invest 109: 41-50, 2002.

300. Iellem A, Mariani M, Lang R, Recalde H, Panina-Bordignon P, Sinigaglia F, and D'Ambrosio D. Unique chemotactic response profile and specific expression of chemokine receptors CCR4 and CCR8 by CD4(+)CD25(+) regulatory T cells. J Exp Med 194: 847-853, 2001.

301. Ignatowski A. Wirkung de tierischen Nahrung auf den Kaninchenorganismus. Ber Milit-med Akad 16: 154-176, 1908

302. Imai T, Hieshima K, Haskell C, Baba M, Nagira M, Nishimura M, Kakizaki M, Takagi S, Nomiyama H, Schall TJ, and Yoshie o. Identification and molecular characterization of fractalkine receptor CX3CR1, which mediates both leukocyte migration and adhesion. Cell 91: 521-530, 1997.

303. Imler JL and Zheng L. Biology of Toll receptors: lessons from insects and mammals. J Leukoc Biol 75: 18-26, 2004

304. Ishibashi S, Brown MS, Goldstein JL, Gerard RD, Hammer RE, and Herz J. Hypercholesterolemia in low density lipoprotein receptor knockout mice and its reversal by adenovirus-mediated gene delivery. J Clin Invest 92: 883-893, 1993.

305. Ishibashi S, Goldstein JL, Brown MS, Herz J, and Burns DK. Massive xanthomatosis and atherosclerosis in cholesterol-fed low density lipoprotein receptor-negative mice. J Clin Invest 93: 18851893, 1994.

306. Ishibashi S, Herz J, Maeda N, Goldstein JL, and Brown MS. The two-receptor model of lipoprotein clearance: tests of the hypothesis in "knockout" mice lacking the low density lipoprotein receptor, apolipoprotein E, or both proteins. Proc Natl Acad Sci USA 91: 4431-4435, 1994.

307. Isoda K, Sawada S, Ishigami N, Matsuki T, Miyazaki K, Kusuhara M, Iwakura Y, and Ohsuzu F. Lack of interleukin-1 receptor antagonist modulates plaque composition in apolipoprotein E-deficient mice. Arterioscler Thromb Vasc Biol 24: 1068-1073, 2004.

308. Ito A, Mukaiyama A, Itoh Y, Nagase H, Thogersen IB, Enghild JJ, Sasaguri Y, and Mori Y. Degradation of interleukin 1beta by matrix metalloproteinases. J Biol Chem 271: 14657-14660, 1996.

309. Jacoby JJ, Kalinowski A, Liu MG, Zhang SS, Gao Q, Chai GX, Ji L, Iwamoto Y, Li E, Schneider M, Russell KS, and Fu XY. Cardiomyocyte-restricted knockout of STAT3 results in higher sensitivity to inflammation, cardiac fibrosis, and heart failure with advanced age. Proc Natl Acad Sci USA 100: 12929-12934, 2003.

310. Janabi M, Yamashita S, Hirano K, Sakai N, Hiraoka H, Matsumoto K, Zhang Z, Nozaki S, and Matsuzawa Y. Oxidized LDL-induced NF-kappa B activation and subsequent expression of proinflammatory genes are defective in monocyte-derived macrophages from CD36-deficient patients. Arterioscler Thromb Vasc Biol 20: 1953-1960, 2000.

311. Janssen-Heininger YM, Poynter ME, and Baeuerle PA. Recent advances towards understanding redox mechanisms in the activation of nuclear factor $\kappa$ B. Free Radic Biol Med 28: 1317-1327, 2000.

312. Janssens S and Beyaert R. A universal role for MyD88 in TLR/ IL-1R-mediated signaling. Trends Biochem Sci 27: 474-482, 2002.

313. Jialal I, Devaraj S, and Venugopal SK. C-reactive protein: risk marker or mediator in atherothrombosis? Hypertension 44: 6-11, 2004.

314. Jin ZG, Lungu AO, Xie L, Wang M, Wong C, and Berk BC. Cyclophilin A is a proinflammatory cytokine that activates endothelial cells. Arterioscler Thromb Vasc Biol 24: 1186-1191, 2004.

315. Johnson GB, Brunn GJ, Kodaira Y, and Platt JL. Receptormediated monitoring of tissue well-being via detection of soluble heparan sulfate by Toll-like receptor 4. J Immunol 168: 5233-5239, 2002.

316. Jonasson L, Holm J, Skalli O, Bondjers G, and Hansson G. Regional accumulations of T cells, macrophages, and smooth muscle cells in the human atherosclerotic plaque. Atherosclerosis 6: 131-138, 1986.

317. Jonasson L, Holm J, Skalli O, Gabbiani G, and Hansson GK. Expression of class II transplantation antigen on vascular smooth muscle cells in human atherosclerosis. J Clin Invest 76: 125-131, 1985.
318. Jones A and Geczy CL. Thrombin and factor Xa enhance the production of interleukin-1. Immunology 71: 236-241, 1990.

319. Kaartinen M, Penttila A, and Kovanen PT. Accumulation of activated mast cells in the shoulder region of human coronary atheroma, the predilection site of atheromatous rupture. Circulation 90: 1669-1678, 1994.

320. Kalinina N, Agrotis A, Antropova Y, Ilyinskaya O, Smirnov V, Tararak E, and Bobik A. Smad expression in human atherosclerotic lesions: evidence for impaired TGF-beta/Smad signaling in smooth muscle cells of fibrofatty lesions. Arterioscler Thromb Vasc Biol 24: 1391-1396, 2004.

321. Kannel WB. Fifty years of Framingham Study contributions to understanding hypertension. J Hum Hypertens 14: 83-90, 2000.

322. Kano A, Wolfgang MJ, Gao Q, Jacoby J, Chai GX, Hansen W, Iwamoto Y, Pober JS, Flavell RA, and Fu XY. Endothelial cells require STAT3 for protection against endotoxin-induced inflammation. J Exp Med 198: 1517-1525, 2003.

323. Kanters E, Gijbels MJ, van der Made I, Vergouwe MN, Heeringa P, Kraal G, Hofker MH, and de Winther MP. Hematopoietic NF-kappaB1 deficiency results in small atherosclerotic lesions with an inflammatory phenotype. Blood 103: 934-940, 2004

324. Kanters E, Pasparakis M, Gijbels MJ, Vergouwe MN, Partouns-Hendriks I, Fijneman RJ, Clausen BE, Forster I, Kockx MM, Rajewsky K, Kraal G, Hofker MH, and de Winther MP. Inhibition of NF-kappaB activation in macrophages increases atherosclerosis in LDL receptor-deficient mice. J Clin Invest 112: $1176-1185,2003$.

325. Kaplan MH, Sun YL, Hoey T, and Grusby MJ. Impaired IL-12 responses and enhanced development of Th2 cells in Stat4-deficient mice. Nature 382: 174-177, 1996.

326. Kaplanski G, Fabrigoule M, Boulay V, Dinarello CA, Bongrand $\mathbf{P}$, Kaplanski S, and Farnarier $\mathbf{C}$. Thrombin induces endothelial type II activation in vitro: IL-1 and TNF-alpha-independent IL-8 secretion and E-selectin expression. J Immunol 158: 54355441, 1997.

327. Kaplanski G, Marin V, Fabrigoule M, Boulay V, Benoliel AM, Bongrand P, Kaplanski S, and Farnarier C. Thrombin-activated human endothelial cells support monocyte adhesion in vitro following expression of intercellular adhesion molecule-1 (ICAM-1; CD54) and vascular cell adhesion molecule-1 (VCAM-1; CD106). Blood 92: 1259-1267, 1998.

328. Karsdal MA, Larsen L, Engsig MT, Lou H, Ferreras M, Lochter A, Delaisse JM, and Foged NT. Matrix metalloproteinase-dependent activation of latent transforming growth factor-beta controls the conversion of osteoblasts into osteocytes by blocking osteoblast apoptosis. J Biol Chem 277: 44061-44067, 2002.

329. Kaukoranta-Tolvanen SS, Ronni T, Leinonen M, Saikku P, and Laitinen K. Expression of adhesion molecules on endothelial cells stimulated by Chlamydia pneumoniae. Microb Pathog 21: 407-411, 1996

330. Kaukoranta-Tolvanen SS, Teppo AM, Laitinen K, Saikku P, Linnavuori K, and Leinonen M. Growth of Chlamydia pneumoniae in cultured human peripheral blood mononuclear cells and induction of a cytokine response. Microb Pathog 21: 215-221, 1996.

331. Kawai T, Adachi O, Ogawa T, Takeda K, and Akira S. Unresponsiveness of MyD88-deficient mice to endotoxin. Immunity 11: 115-122, 1999.

332. Kemper C, Chan AC, Green JM, Brett KA, Murphy KM, and Atkinson JP. Activation of human CD4+ cells with CD3 and CD46 induces a T-regulatory cell 1 phenotype. Nature 421: 388-392, 2003.

333. Keymeulen B, Vandemeulebroucke E, Ziegler AG, Mathieu C, Kaufman L, Hale G, Gorus F, Goldman M, Walter M, Candon S, Schandene L, Crenier L, De Block C, Seigneurin JM, De Pauw P, Pierard D, Weets I, Rebello P, Bird P, Berrie E, Frewin M, Waldmann H, Bach JF, Pipeleers D, and Chatenoud L. Insulin needs after CD3-antibody therapy in new-onset type 1 diabetes. $N$ Engl J Med 352: 2598-2608, 2005

334. Khattri R, Cox T, Yasayko SA, and Ramsdell F. An essential role for Scurfin in CD4+CD25+ T regulatory cells. Nat Immunol 4: 337-342, 2003.

335. Kiechl S, Lorenz E, Reindl M, Wiedermann CJ, Oberhollenzer F, Bonora E, Willeit J, and Schwartz DA. Toll-like receptor 4 
polymorphisms and atherogenesis. N Engl J Med 347: 185-192, 2002.

336. Kim JA, Berliner JA, and Nadler JL. Angiotensin II increases monocyte binding to endothelial cells. Biochem Biophys Res Commun 226: 862-868, 1996.

337. Kim JA, Territo MC, Wayner E, Carlos TM, Parhami F, Smith CW, Haberland ME, Fogelman AM, and Berliner JA. Partial characterization of leukocyte binding molecules on endothelial cells induced by minimally oxidized LDL. Arterioscler Thromb 14: 427-433, 1994.

338. Kim SH, Han SY, Azam T, Yoon DY, and Dinarello CA. Interleukin-32: a cytokine and inducer of TNFalpha. Immunity 22: 131-142, 2005.

339. Kim YM, Talanian RV, Li J, and Billiar TR. Nitric oxide prevents IL-1beta and IFN-gamma-inducing factor (IL-18) release from macrophages by inhibiting caspase-1 (IL-1beta-converting enzyme). J Immunol 161: 4122-4128, 1998.

340. King VL, Szilvassy SJ, and Daugherty A. Interleukin-4 deficiency decreases atherosclerotic lesion formation in a site-specific manner in female LDL receptor-/- mice. Arterioscler Thromb Vasc Biol 22: 456-461, 2002.

341. Kinoshita M, Okada M, Hara M, Furukawa Y, and Matsumori A. Mast cell tryptase in mast cell granules enhances MCP-1 and interleukin-8 production in human endothelial cells. Arterioscler Thromb Vasc Biol 2005.

342. Kirii H, Niwa T, Yamada Y, Wada H, Saito K, Iwakura Y, Asano M, Moriwaki H, and Seishima M. Lack of interleukin-1beta decreases the severity of atherosclerosis in ApoE-deficient mice. Arterioscler Thromb Vasc Biol 23: 656-660, 2003

343. Kislinger T, Tanji N, Wendt T, Qu W, Lu Y, Ferran LJ Jr, Taguchi A, Olson K, Bucciarelli L, Goova M, Hofmann MA, Cataldegirmen G, D'Agati V, Pischetsrieder M, Stern DM, and Schmidt AM. Receptor for advanced glycation end products mediates inflammation and enhanced expression of tissue factor in vasculature of diabetic apolipoprotein E-null mice. Arterioscler Thromb Vasc Biol 21: 905-910, 2001.

344. Klein NJ, Shennan GI, Heyderman RS, and Levin M. Alteration in glycosaminoglycan metabolism and surface charge on human umbilical vein endothelial cells induced by cytokines, endotoxin and neutrophils. J Cell Sci 102: 821-832, 1992.

345. Knowles JW and Maeda N. Genetic modifiers of atherosclerosis in mice. Arterioscler Thromb Vasc Biol 20: 2336-2345, 2000.

346. Knowles JW, Reddick RL, Jennette JC, Shesely EG, Smithies O, and Maeda N. Enhanced atherosclerosis and kidney dysfunction in eNOS( $(-/-)$ Apoe $(-/-)$ mice are ameliorated by enalapril treatment. J Clin Invest 105: 451-458, 2000.

347. Kockx MM, De Meyer GR, Buyssens N, Knaapen MW, Bult H, and Herman AG. Cell composition, replication, and apoptosis in atherosclerotic plaques after 6 months of cholesterol withdrawal. Circ Res 83: 378-387, 1998

348. Kockx MM and Herman AG. Apoptosis in atherosclerosis: beneficial or detrimental? Cardiovasc Res 45: 736-746, 2000

349. Kol A, Bourcier T, Lichtman AH, and Libby P. Chlamydial and human heat shock protein 60 s activate human vascular endothelium, smooth muscle cells, and macrophages. J Clin Invest 103: 571-577, 1999.

350. Kol A and Libby P. The mechanisms by which infectious agents may contribute to atherosclerosis and its clinical manifestations. Trends Cardiovasc Med 8: 191-199, 1998.

351. Kol A, Sukhova GK, Lichtman AH, and Libby P. Chlamydial heat shock protein 60 localizes in human atheroma and regulates macrophage tumor necrosis factor-alpha and matrix metalloproteinase expression. Circulation 98: 300-307, 1998.

352. Kolodgie FD, Gold HK, Burke AP, Fowler DR, Kruth HS, Weber DK, Farb A, Guerrero LJ, Hayase M, Kutys R, Narula J, Finn AV, and Virmani R. Intraplaque hemorrhage and progression of coronary atheroma. N Engl J Med 349: 2316-2325, 2003.

353. Kolodgie FD, Narula J, Burke AP, Haider N, Farb A, HuiLiang Y, Smialek J, and Virmani R. Localization of apoptotic macrophages at the site of plaque rupture in sudden coronary death. Am J Pathol 157: 1259-1268, 2000.

354. Korn SH, Wouters EF, Vos N, and Janssen-Heininger YM. Cytokine-induced activation of nuclear factor-kappa B is inhibited by hydrogen peroxide through oxidative inactivation of IkappaB kinase. J Biol Chem 276: 35693-35700, 2001.

355. Krutzik SR, Tan B, Li H, Ochoa MT, Liu PT, Sharfstein SE, Graeber TG, Sieling PA, Liu YJ, Rea TH, Bloom BR, and Modlin RL. TLR activation triggers the rapid differentiation of monocytes into macrophages and dendritic cells. Nat Med 11: 653-660, 2005.

356. Kugiyama K, Ota Y, Takazoe K, Moriyama Y, Kawano H, Miyao Y, Sakamoto T, Soejima H, Ogawa H, Doi H, Sugiyama $\mathbf{S}$, and Yasue H. Circulating levels of secretory type II phospholipase $\mathrm{A}(2)$ predict coronary events in patients with coronary artery disease. Circulation 100: 1280-1284, 1999

357. Kuhlencordt PJ, Chen J, Han F, Astern J, and Huang PL. Genetic deficiency of inducible nitric oxide synthase reduces atherosclerosis and lowers plasma lipid peroxides in apolipoprotein E-knockout mice. Circulation 103: 3099-3104, 2001.

358. Kuida K, Lippke JA, Ku G, Harding MW, Livingston DJ, Su MSS, and Flavell RA. Altered cytokine export and apoptosis in mice deficient in interleukin-1 $\beta$ converting enzyme. Science 267: 2000-2003, 1995.

359. Kuiper J, von der Thüsen J, de Vos P, Fekkes ML, van Snick J, Biessen EAL, and van Berkel T. Interleukin 9 treatment of LDL receptor deficient mice inhibits atherosclerotic plaque formation (Abstract). Circulation 104: II-320, 2001.

360. Kulkarni AB, Ward JM, Yaswen L, Mackall CL, Bauer SR, Huh CG, Gress RE, and Karlsson S. Transforming growth factor- $\beta 1$ null mice. An animal model for inflammatory disorders. Am J Pathol 146: 264-275, 1995.

361. Kumada M, Kihara S, Ouchi N, Kobayashi H, Okamoto Y, Ohashi K, Maeda K, Nagaretani H, Kishida K, Maeda N, Nagasawa A, Funahashi T, and Matsuzawa Y. Adiponectin specifically increased tissue inhibitor of metalloproteinase-1 through interleukin-10 expression in human macrophages. Circulation 109: 2046-2049, 2004

362. Kume N, Cybulsky MI, and Gimbrone MA Jr. Lysophosphatidylcholine, a component of atherogenic lipoproteins, induces mononuclear leukocyte adhesion molecules in cultured human and rabbit arterial endothelial cells. J Clin Invest 90: 1138-1144, 1992.

363. Kume N and Kita T. Roles of lectin-like oxidized LDL receptor-1 and its soluble forms in atherogenesis. Curr Opin Lipidol 12: 419-423, 2001

364. Kundig TM, Schorle H, Bachmann MF, Hengartner H, Zinkernagel RM, and Horak I. Immune responses in interleukin-2-deficient mice. Science 262: 1059-1061, 1993.

365. Kuziel WA, Dawson TC, Quinones M, Garavito E, Chenaux G, Ahuja SS, Reddick RL, and Maeda N. CCR5 deficiency is not protective in the early stages of atherogenesis in apoE knockout mice. Atherosclerosis 167: 25-32, 2003.

366. Kwak B, Mulhaupt F, Myit S, and Mach F. Statins as a newly recognized type of immunomodulator. Nat Med 6: 1399-1402, 2000.

367. Laine P, Kaartinen M, Penttila A, Panula P, Paavonen T, and Kovanen PT. Association between myocardial infarction and the mast cells in the adventitia of the infarct-related coronary artery. Circulation 99: 361-369, 1999.

368. Lan HY, Bacher M, Yang N, Mu W, Nikolic-Paterson DJ, Metz C, Meinhardt A, Bucala R, and Atkins RC. The pathogenic role of macrophage migration inhibitory factor in immunologically induced kidney disease in the rat. J Exp Med 185: 1455-1465, 1997.

369. Langer JA, Cutrone EC, and Kotenko S. The class II cytokine receptor (CRF2) family: overview and patterns of receptor-ligand interactions. Cytokine Growth Factor Rev 15: 33-48, 2004.

370. Lapidot T and Petit I. Current understanding of stem cell mobilization: the roles of chemokines, proteolytic enzymes, adhesion molecules, cytokines, and stromal cells. Exp Hematol 30: 973-981, 2002.

371. Larche M and Wraith DC. Peptide-based therapeutic vaccines for allergic and autoimmune diseases. Nat Med 11: S69-S76, 2005.

372. Lau DC, Dhillon B, Yan H, Szmitko PE, and Verma S. Adipokines: molecular links between obesity and atheroslcerosis. Am J Physiol Heart Circ Physiol 288: H2031-H2041, 2005.

373. Laurat E, Poirier B, Tupin E, Caligiuri G, Hansson GK, Bariety J, and Nicoletti A. In vivo downregulation of T helper cell 1 
immune responses reduces atherogenesis in apolipoprotein E-knockout mice. Circulation 104: 197-202, 2001.

374. Lawrence T, Gilroy DW, Colville-Nash PR, and Willoughby DA. Possible new role for NF-kappaB in the resolution of inflammation. Nat Med 7: 1291-1297, 2001.

375. Lee EG, Boone DL, Chai S, Libby SL, Chien M, Lodolce JP, and Ma A. Failure to regulate TNF-induced NF-kappaB and cell death responses in A20-deficient mice. Science 289: 2350-2354, 2000.

376. Lee H, Shi W, Tontonoz P, Wang S, Subbanagounder G, Hedrick CC, Hama S, Borromeo C, Evans RM, Berliner JA, and Nagy L. Role for peroxisome proliferator-activated receptor alpha in oxidized phospholipid-induced synthesis of monocyte chemotactic protein-1 and interleukin- 8 by endothelial cells. Circ Res 87: 516-521, 2000.

377. Lee TS, Yen HC, Pan CC, and Chau LY. The role of interleukin 12 in the development of atherosclerosis in ApoE-deficient mice. Arterioscler Thromb Vasc Biol 19: 734-742, 1999.

378. Lee YW, Hennig B, and Toborek M. Redox-regulated mechanisms of IL-4-induced MCP-1 expression in human vascular endothelial cells. Am J Physiol Heart Circ Physiol 284: H185-H192, 2003.

379. Lee YW, Kuhn H, Hennig B, Neish AS, and Toborek M. IL-4induced oxidative stress upregulates VCAM-1 gene expression in human endothelial cells. J Mol Cell Cardiol 33: 83-94, 2001.

380. Lehoux S and Tedgui A. Cellular mechanics and gene expression in blood vessels. J Biomech 36: 631-643, 2003.

381. Leibowitz J. The History of Coronary Disease. London: Wellcome Institute of the History of Medicine, 1970.

382. Leitinger N. Oxidized phospholipids as modulators of inflammation in atherosclerosis. Curr Opin Lipidol 14: 421-430, 2003.

383. Lemarie CA, Esposito B, Tedgui A, and Lehoux S. Pressureinduced vascular activation of nuclear factor-kappaB: role in cell survival. Circ Res 93: 207-212, 2003.

384. Leskinen MJ, Kovanen PT, and Lindstedt KA. Regulation of smooth muscle cell growth, function and death in vitro by activated mast cells: a potential mechanism for the weakening and rupture of atherosclerotic plaques. Biochem Pharmacol 66: 1493-1498, 2003.

385. Lesnik P, Haskell CA, and Charo IF. Decreased atherosclerosis in CX3CR $1-/-$ mice reveals a role for fractalkine in atherogenesis. $J$ Clin Invest 111: 333-340, 2003.

386. Leung BP, Sattar N, Crilly A, Prach M, McCarey DW, Payne H, Madhok R, Campbell C, Gracie JA, Liew FY, and McInnes IB. A novel anti-inflammatory role for simvastatin in inflammatory arthritis. J Immunol 170: 1524-1530, 2003.

387. Li AC and Glass CK. The macrophage foam cell as a target for therapeutic intervention. Nat Med 8: 1235-1242, 2002.

388. Li H, Freeman MW, and Libby P. Regulation of smooth muscle cell scavenger receptor expression in vivo by atherogenic diets and in vitro by cytokines. J Clin Invest 95: 122-133, 1995.

389. Li J, Post M, Volk R, Gao Y, Li M, Metais C, Sato K, Tsai J, Aird W, Rosenberg RD, Hampton TG, Sellke F, Carmeliet P, and Simons M. PR39, a peptide regulator of angiogenesis. Nat Med 6: 49-55, 2000.

390. Li P, Allen H, Banerjee S, Franklin S, Herzog L, Johnston C, McDowell J, Paskind M, Rodman L, Salfeld J, Towne E, Tracey D, Wardwell S, Wei FY, Wong W, Kamen R, and Seshadri T. Mice deficient in IL- $1 \beta$-converting enzyme are defective in production of mature IL-1 $\beta$ and resistant to endotoxic shock. Cell 80: 401-411, 1995.

391. Liao F, Andalibi A, deBeer FC, Fogelman AM, and Lusis AJ. Genetic control of inflammatory gene induction and NF-kB-like transcription factor activation in response to atherogenic diet in mice. J Clin Invest 91: 2572-2579, 1993.

392. Liao F, Berliner JA, Mehrabian M, Navab M, Demer LL, Lusis AJ, and Fogelman AM. Minimally modified low density lipoprotein is biologically active in vivo in mice. J Clin Invest 87: 22532257, 1991.

393. Liao HS, Matsumoto A, Itakura H, Doi T, Honda M, Kodama T, and Geng YJ. Transcriptional inhibition by interleukin- 6 of the class A macrophage scavenger receptor in macrophages derived from human peripheral monocytes and the THP-1 monocytic cell line. Arterioscler Thromb Vasc Biol 19: 1872-1880, 1999.
394. Libby P. Current concepts of the pathogenesis of the acute coronary syndromes. Circulation 104: 365-372, 2001.

395. Libby P. Inflammation in atherosclerosis. Nature 420: 868-874, 2002.

396. Libby P, Egan D, and Skarlatos S. Roles of infectious agents in atherosclerosis and restenosis: an assessment of the evidence and need for future research. Circulation 96: 4095-4103, 1997.

397. Libby $\mathbf{P}$ and Li H. Vascular cell adhesion molecule-1 and smooth muscle cell activation during atherogenesis. J Clin Invest 92: $538-$ 539, 1993.

398. Libby P and Ridker PM. Inflammation and atherosclerosis: role of C-reactive protein in risk assessment. Am J Med $116 \mathrm{Suppl}$ 6A: 9S-16S, 2004

399. Libby $\mathbf{P}$ and Theroux P. Pathophysiology of coronary artery disease. Circulation 111: 3481-3488, 2005.

400. Libby P, Warner SJ, and Friedman GB. Interleukin 1: a mitogen for human vascular smooth muscle cells that induces the release of growth-inhibitory prostanoids. J Clin Invest 81: 487-498, 1988.

401. Lin SG, Yu XY, Chen YX, Huang XR, Metz C, Bucala R, Lau CP, and Lan HY. De novo expression of macrophage migration inhibitory factor in atherogenesis in rabbits. Circ Res 87: 1202-1208, 2000.

402. Lindemann S, Tolley ND, Dixon DA, McIntyre TM, Prescott SM, Zimmerman GA, and Weyrich AS. Activated platelets mediate inflammatory signaling by regulated interleukin 1beta synthesis. J Cell Biol 154: 485-490, 2001.

403. Lindmark E, Diderholm E, Wallentin L, and Siegbahn A. Relationship between interleukin 6 and mortality in patients with unstable coronary artery disease: effects of an early invasive or noninvasive strategy. JAMA 286: 2107-2113, 2001.

404. Lindstedt KA and Kovanen PT. Mast cells in vulnerable coronary plaques: potential mechanisms linking mast cell activation to plaque erosion and rupture. Curr Opin Lipidol 15: 567-573, 2004.

405. Littlewood TD and Bennett MR. Apoptotic cell death in atherosclerosis. Curr Opin Lipidol 14: 469-475, 2003.

406. Liu C, Wang S, Deb A, Nath KA, Katusic ZS, McConnell JP, and Caplice NM. Proapoptotic, antimigratory, antiproliferative, and antiangiogenic effects of commercial C-reactive protein on various human endothelial cell types in vitro: implications of contaminating presence of sodium azide in commercial preparation. Circ Res 97: 135-143, 2005.

407. Liu-Wu Y, Hurt-Camejo E, and Wiklund O. Lysophosphatidylcholine induces the production of IL-1beta by human monocytes. Atherosclerosis 137: 351-357, 1998

408. Liuzzo G, Biasucci LM, Gallimore JR, Grillo RL, Rebuzzi AG, Pepys MB, and Maseri A. The prognostic value of C-reactive protein and serum amyloid a protein in severe unstable angina. N Engl J Med 331: 417-424, 1994.

409. Liuzzo G, Kopecky SL, Frye RL, O'Fallon WM, Maseri A, Goronzy JJ, and Weyand CM. Perturbation of the T-cell repertoire in patients with unstable angina. Circulation 100: 2135-2139, 1999.

410. Llodra J, Angeli V, Liu J, Trogan E, Fisher EA, and Randolph GJ. Emigration of monocyte-derived cells from atherosclerotic lesions characterizes regressive, but not progressive, plaques. Proc Natl Acad Sci USA 101: 11779-11784, 2004.

411. Loffreda S, Yang SQ, Lin HZ, Karp CL, Brengman ML, Wang DJ, Klein AS, Bulkley GB, Bao C, Noble PW, Lane MD, and Diehl AM. Leptin regulates proinflammatory immune responses. FASEB J 12: 57-65, 1998.

412. Lopez AD and Murray CC. The global burden of disease, 19902020. Nat Med 4: 1241-1243, 1998 .

413. Loppnow $\mathbf{H}$ and Libby P. Proliferating or interleukin 1-activated human vascular smooth muscle cells secrete copious interleukin 6 . $J$ Clin Invest 85: 731-738, 1990.

414. Lord GM, Matarese G, Howard JK, Baker RJ, Bloom SR, and Lechler RI. Leptin modulates the T-cell immune response and reverses starvation-induced immunosuppression. Nature 394: 897901, 1998.

415. Lovato P, Brender C, Agnholt J, Kelsen J, Kaltoft K, Svejgaard A, Eriksen KW, Woetmann A, and Odum N. Constitutive STAT3 activation in intestinal T cells from patients with Crohn's disease. J Biol Chem 278: 16777-16781, 2003. 
416. Luscinskas FW, Kansas GS, Ding H, Pizcueta P, Schleiffenbaum BE, Tedder TF, and Gimbrone MA. Monocyte rolling, arrest and spreading on IL-4-activated vascular endothelium under flow is mediated via sequential action of L-selectin, beta(1)-integrins, and beta(2)-integrins. J Cell Biol 125: 1417-1427, 1994.

417. Lusis AJ. Atherosclerosis. Nature 407: 233-241, 2000.

418. Lutgens E, Cleutjens KB, Heeneman S, Koteliansky VE, Burkly LC, and Daemen MJ. Both early and delayed anti-CD40L antibody treatment induces a stable plaque phenotype. Proc Natl Acad Sci USA 97: 7464-7469, 2000.

419. Lutgens E and Daemen MJ. Transforming growth factor-beta: a local or systemic mediator of plaque stability? Circ Res 89: 853855,2001

420. Lutgens E, Gijbels M, Smook M, Heeringa P, Gotwals P, Koteliansky VE, and Daemen MJ. Transforming growth factor-beta mediates balance between inflammation and fibrosis during plaque progression. Arterioscler Thromb Vasc Biol 22: 975-982, 2002.

421. Lutgens E, Gorelik L, Daemen MJ, de Muinck ED, Grewal IS, Koteliansky VE, and Flavell RA. Requirement for CD154 in the progression of atherosclerosis. Nat Med 5: 1313-1316, 1999.

422. Lutgens E, van Suylen RJ, Faber BC, Gijbels MJ, Eurlings PM, Bijnens AP, Cleutjens KB, Heeneman S, and Daemen MJ. Atherosclerotic plaque rupture: local or systemic process? Arterioscler Thromb Vasc Biol 23: 2123-2130, 2003.

423. Luttun A, Tjwa M, Moons L, Wu Y, Angelillo-Scherrer A, Liao F, Nagy JA, Hooper A, Priller J, De Klerck B, Compernolle V, Daci E, Bohlen P, Dewerchin M, Herbert JM, Fava R, Matthys P, Carmeliet G, Collen D, Dvorak HF, Hicklin DJ, and Carmeliet P. Revascularization of ischemic tissues by PlGF treatment, and inhibition of tumor angiogenesis, arthritis and atherosclerosis by anti-Flt1. Nat Med 8: 831-840, 2002.

424. Mach F, Sauty A, Iarossi AS, Sukhova GK, Neote K, Libby P, and Luster AD. Differential expression of three T lymphocyteactivating CXC chemokines by human atheroma-associated cells. J Clin Invest 104: 1041-1050, 1999.

425. Mach F, Schonbeck U, Fabunmi RP, Murphy C, Atkinson E, Bonnefoy JY, Graber P, and Libby P. T lymphocytes induce endothelial cell matrix metalloproteinase expression by a CD40Ldependent mechanism: implications for tubule formation. Am J Pathol 154: 229-238, 1999.

426. Mach F, Schonbeck U, Sukhova GK, Atkinson E, and Libby P. Reduction of atherosclerosis in mice by inhibition of CD40 signalling. Nature 394: 200-203, 1998.

427. MacKenzie A, Wilson HL, Kiss-Toth E, Dower SK, North RA, and Surprenant A. Rapid secretion of interleukin-1beta by microvesicle shedding. Immunity 15: 825-835, 2001.

428. Maddox JF and Serhan CN. Lipoxin A4 and B4 are potent stimuli for human monocyte migration and adhesion: selective inactivation by dehydrogenation and reduction. J Exp Med 183: 137-146, 1996.

429. Maeda S, Dean DD, Gomez R, Schwartz Z, and Boyan BD. The first stage of transforming growth factor betal activation is release of the large latent complex from the extracellular matrix of growth plate chondrocytes by matrix vesicle stromelysin-1 (MMP-3). Calcif Tissue Int 70: 54-65, 2002.

430. Major AS, Wilson MT, McCaleb JL, Ru Su Y, Stanic AK, Joyce S, Van Kaer L, Fazio S, and Linton MF. Quantitative and qualitative differences in proatherogenic NKT cells in apolipoprotein E-deficient mice. Arterioscler Thromb Vasc Biol 24: 2351-2357, 2004.

431. Maldonado-Lopez R, De Smedt T, Michel P, Godfroid J, Pajak B, Heirman C, Thielemans K, Leo O, Urbain J, and Moser M. CD8alpha + and CD8alpha - subclasses of dendritic cells direct the development of distinct T helper cells in vivo. J Exp Med 189: 587-592, 1999.

432. Malek TR. The main function of IL-2 is to promote the development of T regulatory cells. J Leukoc Biol 74: 961-965, 2003.

433. Mallat Z, Benamer H, Hugel B, Benessiano J, Steg PG, Freyssinet JM, and Tedgui A. Elevated levels of shed membrane microparticles with procoagulant potential in the peripheral circulating blood of patients with acute coronary syndromes. Circulation 101: 841-843, 2000

434. Mallat Z, Besnard S, Duriez M, Deleuze V, Emmanuel F, Bureau MF, Soubrier F, Esposito B, Duez H, Fievet C, Staels B,
Duverger N, Scherman D, and Tedgui A. Protective role of interleukin-10 in atherosclerosis. Circ Res 85: e17-e24, 1999.

435. Mallat Z, Corbaz A, Scoazec A, Besnard S, Leseche G, Chvatchko Y, and Tedgui A. Expression of interleukin-18 in human atherosclerotic plaques and relation to plaque instability. Circulation 104: 1598-1603, 2001.

436. Mallat Z, Corbaz A, Scoazec A, Graber P, Alouani S, Esposito B, Humbert Y, Chvatchko Y, and Tedgui A. Interleukin-18/ interleukin-18 binding protein signaling modulates atherosclerotic lesion development and stability. Circ Res 89: E41-E45, 2001.

437. Mallat Z, Gojova A, Brun V, Esposito B, Fournier N, Cottrez $\mathbf{F}$, Tedgui $\mathbf{A}$, and Groux $\mathbf{H}$. Induction of a regulatory T cell type 1 response reduces the development of atherosclerosis in apolipoprotein E-knockout mice. Circulation 108: 1232-1237, 2003.

438. Mallat Z, Gojova A, Marchiol-Fournigault C, Esposito B, Kamate C, Merval R, Fradelizi D, and Tedgui A. Inhibition of transforming growth factor-beta signaling accelerates atherosclerosis and induces an unstable plaque phenotype in mice. Circ Res 89: 930-934, 2001.

439. Mallat Z, Henry P, Fressonnet R, Alouani S, Scoazec A, Beaufils P, Chvatchko Y, and Tedgui A. Increased plasma concentrations of interleukin-18 in acute coronary syndromes. Heart 88: 467-469, 2002.

440. Mallat Z, Heymes C, Corbaz A, Logeart D, Alouani S, CohenSolal A, Seidler T, Hasenfuss G, Chvatchko Y, Shah AM, and Tedgui A. Evidence for altered interleukin 18 (IL)-18 pathway in human heart failure. FASEB $J$ 18: 1752-1754, 2004.

441. Mallat Z, Heymes C, Ohan J, Faggin E, Lesèche G, and Tedgui A. Expression of interleukin-10 in human atherosclerotic plaques. Relation to inducible nitric oxide synthase expression and cell death. Arterioscler Thromb Vasc Biol 19: 611-616, 1999.

442. Mallat Z, Hugel B, Ohan J, Leseche G, Freyssinet JM, and Tedgui A. Shed membrane microparticles with procoagulant potential in human atherosclerotic plaques: a role for apoptosis in plaque thrombogenicity. Circulation 99: 348-353, 1999.

443. Mallat $\mathbf{Z}$ and Tedgui A. Current perspective on the role of apoptosis in atherothrombotic disease. Circ Res 88: 998-1003, 2001.

444. Mallat $\mathbf{Z}$ and Tedgui A. Immunomodulation to combat atherosclerosis: the potential role of immune regulatory cells. Exp Opin Biol Ther 4: 1387-1393, 2004.

445. Mallat $\mathbf{Z}$ and Tedgui A. The role of transforming growth factor beta in atherosclerosis: novel insights and future perspectives. Curr Opin Lipidol 13: 523-529, 2002.

446. Mandal K, Jahangiri M, and Xu Q. Autoimmunity to heat shock proteins in atherosclerosis. Autoimmune Rev 3: 31-37, 2004.

447. Mantovani A, Bussolino F, and Introna M. Cytokine regulation of endothelial cell function: from molecular level to the bedside. Immunol Today 18: 231-240, 1997.

448. Marie JC, Astier AL, Rivailler P, Rabourdin-Combe C, Wild TF, and Horvat B. Linking innate and acquired immunity: divergent role of CD46 cytoplasmic domains in T cell induced inflammation. Nat Immunol 3: 659-666, 2002.

449. Marin V, Montero-Julian FA, Gres S, Boulay V, Bongrand P, Farnarier C, and Kaplanski G. The IL-6-soluble IL-6Ralpha autocrine loop of endothelial activation as an intermediate between acute and chronic inflammation: an experimental model involving thrombin. J Immunol 167: 3435-3442, 2001.

450. Maron R, Sukhova G, Faria AM, Hoffmann E, Mach F, Libby $P$, and Weiner HL. Mucosal administration of heat shock protein-65 decreases atherosclerosis and inflammation in aortic arch of lowdensity lipoprotein receptor-deficient mice. Circulation 106: 1708$1715,2002$.

451. Marsman DS, Cattley RC, Conway JG, and Popp JA. Relationship of hepatic peroxisome proliferation and replicative DNA synthesis to the hepatocarcinogenicity of the peroxisome proliferators di(2-ethyl-hexyl)phthalate and [4-chloro-6-(2,3-xylidino)-2-pyrimidinylthio]acetic acid (Wy 14,643) in rats. Cancer Res 48: 6739 6744, 1988.

452. Martinon F and Tschopp J. Inflammatory caspases: linking an intracellular innate immune system to autoinflammatory diseases. Cell 117: 561-574, 2004

453. Massague $\mathbf{J}$ and Wotton $\mathbf{D}$. Transcriptional control by the TGFbeta/Smad signaling system. EMBO J 19: 1745-1754, 2000. 
454. Matarese G, Carrieri PB, La Cava A, Perna F, Sanna V, De Rosa V, Aufiero D, Fontana S, and Zappacosta S. Leptin increase in multiple sclerosis associates with reduced number of CD4(+)CD25+ regulatory T cells. Proc Natl Acad Sci USA 102: $5150-5155,2005$.

455. Matloubian M, David A, Engel S, Ryan JE, and Cyster JG. A transmembrane CXC chemokine is a ligand for HIV-coreceptor Bonzo. Nat Immunol 1: 298-304, 2000.

456. Matsuda H, Ushio H, Geba GP, and Askenase PW. Human platelets can initiate T cell-dependent contact sensitivity through local serotonin release mediated by IgE antibodies. J Immunol 158: 2891-2897, 1997.

457. Mause SF, von Hundelshausen P, Zernecke A, Koenen RR, and Weber C. Platelet microparticles: a transcellular delivery system for RANTES promoting monocyte recruitment on endothelium. Arterioscler Thromb Vasc Biol 25: 1512-1518, 2005.

458. Maziere C, Auclair M, and Maziere JC. Tumor necrosis factor enhances low density lipoprotein oxidative modification by monocytes and endothelial cells. FEBS Lett 338: 43-46, 1994.

459. Mazzolai L, Duchosal M, Korber M, Bouzourene K, Aubert J, Hao H, Vallet V, Brunner H, Nussberger J, Gabbiani G, and Hayoz D. Endogenous angiotensin II induces atherosclerotic plaque vulnerability and elicits a Th1 response in apoE-/- mice. Hypertension 44: 277-282, 2004.

460. McGill HC Jr, McMahan CA, Herderick EE, Zieske AW, Malcom GT, Tracy RE, and Strong JP. Obesity accelerates the progression of coronary atherosclerosis in young men. Circulation 105: 2712-2718, 2002.

461. Mehrabian M, Allayee H, Wong J, Shi W, Wang XP, Shaposhnik Z, Funk CD, and Lusis AJ. Identification of 5-lipoxygenase as a major gene contributing to atherosclerosis susceptibility in mice. Circ Res 91: 120-126, 2002

462. Merhi-Soussi F, Kwak BR, Magne D, Chadjichristos C, Berti M, Pelli G, James RW, Mach F, and Gabay C. Interleukin-1 plays a major role in vascular inflammation and atherosclerosis in male apolipoprotein E-knockout mice. Cardiovasc Res 66: 583-593, 2005.

463. Mertens A, Verhamme P, Bielicki JK, Phillips MC, Quarck R, Verreth W, Stengel D, Ninio E, Navab M, Mackness B, Mackness $M$, and Holvoet $P$. Increased low-density lipoprotein oxidation and impaired high-density lipoprotein antioxidant defense are associated with increased macrophage homing and atherosclerosis in dyslipidemic obese mice: LCAT gene transfer decreases atherosclerosis. Circulation 107: 1640-1646, 2003.

464. Mesri M and Altieri DC. Endothelial cell activation by leukocyte microparticles. J Immunol 161: 4382-4387, 1998.

465. Mesri M and Altieri DC. Leukocyte microparticles stimulate endothelial cell cytokine release and tissue factor induction in a JNK1 signaling pathway. J Biol Chem 274: 23111-23118, 1999.

466. Metcalf D, Mifsud S, Di Rago L, Nicola NA, Hilton DJ, and Alexander WS. Polycystic kidneys and chronic inflammatory lesions are the delayed consequences of loss of the suppressor of cytokine signaling-1 (SOCS-1). Proc Natl Acad Sci USA 99: 943-948, 2002.

467. Meyer G, Merval R, and Tedgui A. Effects of pressure-induced wall stretching and convection on low density lipoprotein and albumin uptake in the rabbit aorta. Circ Res 79: 532-540, 1996.

468. Michelsen KS, Doherty TM, Shah PK, and Arditi M. Role of Toll-like receptors in atherosclerosis. Circ Res 95: e96-e97, 2004.

469. Michelsen KS, Doherty TM, Shah PK, and Arditi M. TLR signaling: an emerging bridge from innate immunity to atherogenesis. J Immunol 173: 5901-5907, 2004.

470. Michelsen KS, Wong MH, Shah PK, Zhang W, Yano J, Doherty TM, Akira S, Rajavashisth TB, and Arditi M. Lack of Toll-like receptor 4 or myeloid differentiation factor 88 reduces atherosclerosis and alters plaque phenotype in mice deficient in apolipoprotein E. Proc Natl Acad Sci USA 101: 10679-10684, 2004.

471. Miller YI, Viriyakosol S, Worrall DS, Boullier A, Butler S, and Witztum JL. Toll-like receptor 4-dependent and -independent cytokine secretion induced by minimally oxidized low-density lipoprotein in macrophages. Arterioscler Thromb Vasc Biol 25: 1213-1219, 2005.
472. Minami M, Kume N, Shimaoka T, Kataoka H, Hayashida K, Akiyama Y, Nagata I, Ando K, Nobuyoshi M, Hanyuu M, Komeda M, Yonehara S, and Kita T. Expression of SR-PSOX, a novel cell-surface scavenger receptor for phosphatidylserine and oxidized LDL in human atherosclerotic lesions. Arterioscler Thromb Vasc Biol 21: 1796-1800, 2001.

473. Mizel SB and Farrar JJ. Revised nomenclature for antigen-nonspecific T-cell proliferation and helper factors. Cell Immunol 48: 433-436, 1979.

474. Mizoguchi A, Mizoguchi E, Takedatsu H, Blumberg RS, and Bhan AK. Chronic intestinal inflammatory condition generates IL-10-producing regulatory B cell subset characterized by CD1d upregulation. Immunity 16: 219-230, 2002.

475. Moazed TC, Campbell LA, Rosenfeld ME, Grayston JT, and Kuo CC. Chlamydia pneumoniae infection accelerates the progression of atherosclerosis in apolipoprotein E-deficient mice. $J$ Infect Dis 180: 238-241, 1999.

476. Modur V, Feldhaus MJ, Weyrich AS, Jicha DL, Prescott SM, Zimmerman GA, and McIntyre TM. Oncostatin M is a proinflammatory mediator. In vivo effects correlate with endothelial cell expression of inflammatory cytokines and adhesion molecules. J Clin Invest 100: 158-168, 1997.

477. Mohan MJ, Seaton T, Mitchell J, Howe A, Blackburn K, Burkhart W, Moyer M, Patel I, Waitt GM, Becherer JD, Moss ML, and Milla ME. The tumor necrosis factor-alpha converting enzyme (TACE): a unique metalloproteinase with highly defined substrate selectivity. Biochemistry 41: 9462-9469, 2002.

478. Mohan S, Mohan N, Valente AJ, and Sprague EA. Regulation of low shear flow-induced HAEC VCAM-1 expression and monocyte adhesion. Am J Physiol Cell Physiol 276: C1100-C1107, 1999.

479. Mold C, Rodriguez W, Rodic-Polic B, and Du Clos TW. C-reactive protein mediates protection from lipopolysaccharide through interactions with Fc gamma R. J Immunol 169: 7019-7025, 2002.

480. Monaco C, Andreakos E, Kiriakidis S, Mauri C, Bicknell C, Foxwell B, Cheshire N, Paleolog E, and Feldmann M. Canonical pathway of nuclear factor kappa B activation selectively regulates proinflammatory and prothrombotic responses in human atherosclerosis. Proc Natl Acad Sci USA 101: 5634-5639, 2004.

481. Moore KW, de Waal Malefyt R, Coffman RL, and O'Garra A. Interleukin-10 and the interleukin-10 receptor. Annu Rev Immunol 19: 683-765, 2001.

482. Morel O, Hugel B, Jesel L, Mallat Z, Lanza F, Douchet MP, Zupan M, Chauvin M, Cazenave JP, Tedgui A, Freyssinet JM, and Toti F. Circulating procoagulant microparticles and soluble GPV in myocardial infarction treated by primary percutaneous transluminal coronary angioplasty. A possible role for GPIIb-IIIa antagonists. J Thromb Haemost 2: 1118-1126, 2004.

483. Moss ML, Jin SL, Milla ME, Bickett DM, Burkhart W, Carter HL, Chen WJ, Clay WC, Didsbury JR, Hassler D, Hoffman CR, Kost TA, Lambert MH, Leesnitzer MA, McCauley P, McGeehan G, Mitchell J, Moyer M, Pahel G, Rocque W, Overton LK, Schoenen F, Seaton T, Su JL, and Becherer JD. Cloning of a disintegrin metalloproteinase that processes precursor tumour-necrosis factor-alpha. Nature 385: 733-736, 1997.

484. Munder M, Mallo M, Eichmann K, and Modolell M. Murine macrophages secrete interferon gamma upon combined stimulation with interleukin (IL)-12 and IL-18: a novel pathway of autocrine macrophage activation. J Exp Med 187: 2103-2108, 1998.

485. Naito Y, Tsujino T, Fujioka Y, Ohyanagi M, Okamura H, and Iwasaki T. Increased circulating interleukin-18 in patients with congestive heart failure. Heart 88: 296-297, 2002.

486. Nakai Y, Iwabuchi K, Fujii S, Ishimori N, Dashtsoodol N, Watano K, Mishima T, Iwabuchi C, Tanaka S, Bezbradica JS, Nakayama T, Taniguchi M, Miyake S, Yamamura T, Kitabatake A, Joyce S, Van Kaer L, and Onoe K. Natural killer T cells accelerate atherogenesis in mice. Blood 104: 2051-2059, 2004.

487. Nakashima Y, Plump AS, Raines EW, Breslow JL, and Ross R. ApoE-deficient mice develop lesions of all phases of atherosclerosis throughout the arterial tree. Arterioscler Thromb 14: 133-140, 1994.

488. Namiki M, Kawashima S, Yamashita T, Ozaki M, Sakoda T, Inoue N, Hirata K, Morishita R, Kaneda Y, and Yokoyama M. Intramuscular gene transfer of interleukin-10 cDNA reduces ath- 
erosclerosis in apolipoprotein E-knockout mice. Atherosclerosis 172: 21-29, 2004.

489. Napoli C, D'Armiento FP, Mancini FP, Postiglione A, Witztum JL, Palumbo G, and Palinski W. Fatty streak formation occurs in human fetal aortas and is greatly enhanced by maternal hypercholesterolemia. Intimal accumulation of low density lipoprotein and its oxidation precede monocyte recruitment into early atherosclerotic lesions. J Clin Invest 100: 2680-2690, 1997.

490. Narins CR, Lin DA, Burton PB, Jin ZG, and Berk BC. Interleukin-18 and interleukin-18 binding protein levels before and after percutaneous coronary intervention in patients with and without recent myocardial infarction. Am J Cardiol 94: 1285-1287, 2004.

491. Nataraj C, Oliverio MI, Mannon RB, Mannon PJ, Audoly LP, Amuchastegui CS, Ruiz P, Smithies O, and Coffman TM. Angiotensin II regulates cellular immune responses through a calcineurin-dependent pathway. J Clin Invest 104: 1693-1701, 1999.

492. Nath N, Giri S, Prasad R, Singh AK, and Singh I. Potential targets of 3-hydroxy-3-methylglutaryl coenzyme A reductase inhibitor for multiple sclerosis therapy. J Immunol 172: 1273-1286, 2004.

493. Nathan C. Points of control in inflammation. Nature 420: 846-852, 2002.

494. Navab M, Ananthramaiah GM, Reddy ST, Van Lenten BJ, Ansell BJ, Fonarow GC, Vahabzadeh K, Hama S, Hough G, Kamranpour N, Berliner JA, Lusis AJ, and Fogelman AM. The oxidation hypothesis of atherogenesis: the role of oxidized phospholipids and HDL. J Lipid Res 45: 993-1007, 2004.

495. Nelken NA, Coughlin SR, Gordon D, and Wilcox JN. Monocyte chemoattractant protein-1 in human atheromatous plaques. J Clin Invest 88: 1121-1127, 1991.

496. Nelson DE, Ihekwaba AE, Elliott M, Johnson JR, Gibney CA, Foreman BE, Nelson G, See V, Horton CA, Spiller DG, Edwards SW, McDowell HP, Unitt JF, Sullivan E, Grimley R, Benson N, Broomhead D, Kell DB, and White MR. Oscillations in NF-kappaB signaling control the dynamics of gene expression. Science 306: 704-708, 2004.

497. Neri Serneri GG, Prisco D, Martini F, Gori AM, Brunelli T, Poggesi L, Rostagno C, Gensini GF, and Abbate R. Acute T-cell activation is detectable in unstable angina. Circulation 95: 1806$1812,1997$.

498. Netea MG, Kullberg BJ, Galama JM, Stalenhoef AF, Dinarello CA, and Van der Meer JW. Non-LPS components of Chlamydia pneumoniae stimulate cytokine production through Toll-like receptor 2-dependent pathways. Eur J Immunol 32: 1188-1195, 2002.

499. Netea MG, Kullberg BJ, Jacobs LE, Verver-Jansen TJ, van der Ven-Jongekrijg J, Galama JM, Stalenhoef AF, Dinarello CA, and Van der Meer JW. Chlamydia pneumoniae stimulates IFN-gamma synthesis through MyD88-dependent, TLR2- and TLR4independent induction of IL-18 release. J Immunol 173: 1477-1482, 2004.

500. Newby AC. Dual role of matrix metalloproteinases (matrixins) in intimal thickening and atherosclerotic plaque rupture. Physiol Rev 85: 1-31, 2005.

501. Ni W, Kitamoto S, Ishibashi M, Usui M, Inoue S, Hiasa K, Zhao Q, Nishida K, Takeshita A, and Egashira K. Monocyte chemoattractant protein-1 is an essential inflammatory mediator in angiotensin II-induced progression of established atherosclerosis in hypercholesterolemic mice. Arterioscler Thromb Vasc Biol 24: 534539, 2004.

502. Nikol S, Isner JM, Pickering JG, Kearney M, Leclerc G, and Weir L. Expression of transforming growth factor-beta 1 is increased in human vascular restenosis lesions. J Clin Invest 90: 1582-1592, 1992.

503. Nishi E, Kume N, Ueno Y, Ochi H, Moriwaki H, and Kita T. Lysophosphatidylcholine enhances cytokine-induced interferon gamma expression in human T lymphocytes. Circ Res 83: 508-515, 1998

504. Nishi T, Shimizu N, Hiramoto M, Sato I, Yamaguchi Y, Hasegawa $M$, Aizawa $S$, Tanaka $H$, Kataoka $K$, Watanabe $H$, and Handa $\mathbf{H}$. Spatial redox regulation of a critical cysteine residue of NF-kappa B in vivo. J Biol Chem 277: 44548-44556, 2002.

505. Nissen SE, Tuzcu EM, Schoenhagen P, Brown BG, Ganz P, Vogel RA, Crowe T, Howard G, Cooper CJ, Brodie B, Grines
CL, and DeMaria AN. Effect of intensive compared with moderate lipid-lowering therapy on progression of coronary atherosclerosis: a randomized controlled trial. JAMA 291: 1071-1080, 2004.

506. Nissen SE, Tuzcu EM, Schoenhagen P, Crowe T, Sasiela WJ, Tsai J, Orazem J, Magorien RD, O'Shaughnessy C, and Ganz P. Statin therapy, LDL cholesterol, C-reactive protein, and coronary artery disease. N Engl J Med 352: 29-38, 2005.

507. Niwa T, Wada H, Ohashi H, Iwamoto N, Ohta H, Kirii H, Fujii $\mathbf{H}$, Saito K, and Seishima M. Interferon-gamma produced by bone marrow-derived cells attenuates atherosclerotic lesion formation in LDLR-deficient mice. J Atheroscler Thromb 11: 79-87, 2004.

508. O'Brien ER, Garvin MR, Stewart DK, Hinohara T, Simpson JB, Schwartz SM, and Giachelli CM. Osteopontin is synthesized by macrophage, smooth muscle, and endothelial cells in primary and restenotic human coronary atherosclerotic plaques. Arterioscler Thromb 14: 1648-1656, 1994.

509. O'Brien KD, Allen MD, McDonald TO, Chait A, Harlan JM, Fishbein D, McCarty J, Ferguson M, Hudkins K, Benjamin CD, Lobb R, and Alpers CE. Vascular cell adhesion molecule-1 is expressed in human coronary atherosclerotic plaques. Implications for the mode of progression of advanced coronary atherosclerosis. $J$ Clin Invest 92: 945-951, 1993.

510. O'Brien KD, McDonald TO, Chait A, Allen MD, and Alpers CE. Neovascular expression of E-selectin, intercellular adhesion molecule-1, and vascular cell adhesion molecule-1 in human atherosclerosis and their relation to intimal leukocyte content. Circulation 93: 672-682, 1996.

511. O'Connor CM, Dunne MW, Pfeffer MA, Muhlestein JB, Yao L, Gupta S, Benner RJ, Fisher MR, and Cook TD. Azithromycin for the secondary prevention of coronary heart disease events: the WIZARD study: a randomized controlled trial. JAMA 290: 14591466, 2003.

512. O'Garra A and Vieira P. Regulatory T cells and mechanisms of immune system control. Nat Med 10: 801-805, 2004.

515. Ohta H, Wada H, Niwa T, Kirii H, Iwamoto N, Fujii H, Saito K, Sekikawa K, and Seishima M. Disruption of tumor necrosis factor-alpha gene diminishes the development of atherosclerosis in ApoE-deficient mice. Atherosclerosis 180: 11-17, 2005.

516. Okamoto Y, Kihara S, Ouchi N, Nishida M, Arita Y, Kumada M, Ohashi K, Sakai N, Shimomura I, Kobayashi H, Terasaka N, Inaba T, Funahashi T, and Matsuzawa Y. Adiponectin reduces atherosclerosis in apolipoprotein E-deficient mice. Circulation 106: 2767-2770, 2002.

517. Okamura Y, Watari M, Jerud ES, Young DW, Ishizaka ST, Rose J, Chow JC, and Strauss JF III. The extra domain A of fibronectin activates Toll-like receptor 4. J Biol Chem 276: 1022910233, 2001

517a.O'Leary DH, Polak JF, Kronmal RA, Manolio TA, Burke GL, and Wolfson SK Jr. Carotid-artery intima and media thickness as a risk factor for myocardial infarction and stroke in older adults. Cardiovascular Health Study Collaborative Research Group. N Engl J Med 340: 14-22, 1999.

518. Olsen NJ and Stein CM. New drugs for rheumatoid arthritis. N Engl J Med 350: 2167-2179, 2004.

519. Oppenheimer-Marks N, Brezinschek RI, Mohamadzadeh M, Vita R, and Lipsky PE. Interleukin 15 is produced by endothelial cells and increases the transendothelial migration of $\mathrm{T}$ cells in vitro and in the SCID mouse-human rheumatoid arthritis model in vivo. $J$ Clin Invest 101: 1261-1272, 1998.

520. Ortego M, Bustos C, Hernandez-Presa MA, Tunon J, Diaz C, Hernandez G, and Egido J. Atorvastatin reduces NF-kappaB activation and chemokine expression in vascular smooth muscle cells and mononuclear cells. Atherosclerosis 147: 253-261, 1999.

520a.O'Shea JJ, Pesu M, Borie DC, and Changelian PS. A new modality for immunosuppression: targeting the JAK/STAT pathway. Nat Rev Drug Discov 3: 555-564, 2004

521. Osterud B and Bjorklid E. Role of monocytes in atherogenesis. Physiol Rev 83: 1069-1112, 2003.

522. Ott VL, Cambier JC, Kappler J, Marrack P, and Swanson BJ. Mast cell-dependent migration of effector CD8 $+\mathrm{T}$ cells through production of leukotriene $\mathrm{B}_{4}$. Nat Immunol 4: 974-981, 2003.

523. Ouchi N, Kihara S, Arita Y, Maeda K, Kuriyama H, Okamoto Y, Hotta K, Nishida M, Takahashi M, Nakamura T, Yamashita S, 
Funahashi T, and Matsuzawa Y. Novel modulator for endothelial adhesion molecules: adipocyte-derived plasma protein adiponectin. Circulation 100: 2473-2476, 1999.

524. Ouchi N, Kihara S, Arita Y, Nishida M, Matsuyama A, Okamoto $Y$, Ishigami M, Kuriyama $H$, Kishida $K$, Nishizawa $H$, Hotta K, Muraguchi M, Ohmoto Y, Yamashita S, Funahashi T, and Matsuzawa Y. Adipocyte-derived plasma protein, adiponectin, suppresses lipid accumulation and class A scavenger receptor expression in human monocyte-derived macrophages. Circulation 103: 1057-1063, 2001.

525. Palinski W, Miller E, and Witztum JL. Immunization of low density lipoprotein (ldl) receptor-deficient rabbits with homologous malondialdehyde-modified LDL reduces atherogenesis. Proc Natl Acad Sci USA 92: 821-825, 1995.

526. Palinski W, Rosenfeld ME, Yla-Herttuala S, Gurtner GC, Socher SS, Butler SW, Parthasarathy S, Carew TE, Steinberg D, and Witztum JL. Low density lipoprotein undergoes oxidative modification in vivo. Proc Natl Acad Sci USA 86: 1372-1376, 1989.

527. Pampou S, Gnedoy SN, Bystrevskaya VB, Smirnov VN, Chazov EI, Melnick JL, and DeBakey ME. Cytomegalovirus genome and the immediate-early antigen in cells of different layers of human aorta. Virchows Arch 436: 539-552, 2000.

528. Pan JH, Sukhova GK, Yang JT, Wang B, Xie T, Fu H, Zhang Y, Satoskar AR, David JR, Metz CN, Bucala R, Fang K, Simon DI, Chapman HA, Libby P, and Shi GP. Macrophage migration inhibitory factor deficiency impairs atherosclerosis in low-density lipoprotein receptor-deficient mice. Circulation 109: 3149-3153, 2004.

529. Pan Y, Lloyd C, Zhou H, Dolich S, Deeds J, Gonzalo JA, Vath J, Gosselin M, Ma J, Dussault B, Woolf E, Alperin G, Culpepper J, Gutierrez-Ramos JC, and Gearing D. Neurotactin, a membrane-anchored chemokine upregulated in brain inflammation. Nature 387: 611-617, 1997.

530. Panousis CG, Evans G, and Zuckerman SH. TGF-beta increases cholesterol efflux and ABC-1 expression in macrophage-derived foam cells: opposing the effects of IFN-gamma. J Lipid Res 42: 856-863, 2001.

531. Park L, Raman KG, Lee KJ, Lu Y, Ferran LJ Jr, Chow WS, Stern D, and Schmidt AM. Suppression of accelerated diabetic atherosclerosis by the soluble receptor for advanced glycation end products. Nat Med 4: 1025-1031, 1998.

532. Parks WC, Wilson CL, and Lopez-Boado YS. Matrix metalloproteinases as modulators of inflammation and innate immunity. Nat Rev Immunol 4: 617-629, 2004.

533. Pasceri V, Cheng JS, Willerson JT, and Yeh ET. Modulation of C-reactive protein-mediated monocyte chemoattractant protein-1 induction in human endothelial cells by anti-atherosclerosis drugs. Circulation 103: 2531-2534, 2001.

534. Pasceri V, Willerson JT, and Yeh ET. Direct proinflammatory effect of C-reactive protein on human endothelial cells. Circulation 102: 2165-2168, 2000.

535. Pastore L, Tessitore A, Martinotti S, Toniato E, Alesse E, Bravi MC, Ferri C, Desideri G, Gulino A, and Santucci A. Angiotensin II stimulates intercellular adhesion molecule-1 (ICAM-1) expression by human vascular endothelial cells and increases soluble ICAM-1 release in vivo. Circulation 100: 16461652, 1999.

536. Patel L, Buckels AC, Kinghorn IJ, Murdock PR, Holbrook JD, Plumpton C, Macphee CH, and Smith SA. Resistin is expressed in human macrophages and directly regulated by PPAR gamma activators. Biochem Biophys Res Commun 300: 472-476, 2003.

537. Patella V, Marino I, Arbustini E, Lamparter-Schummert B, Verga L, Adt M, and Marone G. Stem cell factor in mast cells and increased mast cell density in idiopathic and ischemic cardiomyopathy. Circulation 97: 971-978, 1998.

538. Paul A, Ko KW, Li L, Yechoor V, McCrory MA, Szalai AJ, and Chan L. C-reactive protein accelerates the progression of atherosclerosis in apolipoprotein E-deficient mice. Circulation 109: 647655, 2004

539. Pepys MB, Hawkins PN, Kahan MC, Tennent GA, Gallimore JR, Graham D, Sabin CA, Zychlinsky A, and de Diego J. Proinflammatory effects of bacterial recombinant human C-reac- tive protein are caused by contamination with bacterial products, not by C-reactive protein itself. Circ Res 97: e97-e103, 2005.

540. Piek E, Heldin CH, and Ten Dijke P. Specificity, diversity, and regulation in TGF-beta superfamily signaling. FASEB $J$ 13: 21052124, 1999.

541. Pinderski LJ, Fischbein MP, Subbanagounder G, Fishbein MC, Kubo N, Cheroutre H, Curtiss LK, Berliner JA, and Boisvert WA. Overexpression of interleukin-10 by activated T lymphocytes inhibits atherosclerosis in LDL receptor-deficient mice by altering lymphocyte and macrophage phenotypes. Circ Res 90: $1064-1071,2002$

542. Pinderski Oslund LJ, Hedrick CC, Olvera T, Hagenbaugh A, Territo M, Berliner JA, and Fyfe AI. Interleukin-10 blocks atherosclerotic events in vitro and in vivo. Arterioscler Thromb Vasc Biol 19: 2847-2853, 1999.

543. Plump AS, Smith JD, Hayek T, Aaltosetala K, Walsh A, Verstuyft JG, Rubin EM, and Breslow JL. Severe hypercholesterolemia and atherosclerosis in apolipoprotein-E-deficient mice created by homologous recombination in ES cells. Cell 71: 343-353, 1992.

544. Pockley AG. Heat shock proteins, inflammation, and cardiovascular disease. Circulation 105: 1012-1017, 2002.

545. Pockley AG, Georgiades A, Thulin T, de Faire U, and Frostegard J. Serum heat shock protein 70 levels predict the development of atherosclerosis in subjects with established hypertension. Hypertension 42: 235-238, 2003.

546. Pockley AG, Wu R, Lemne C, Kiessling R, de Faire U, and Frostegard J. Circulating heat shock protein 60 is associated with early cardiovascular disease. Hypertension 36: 303-307, 2000.

547. Poole JC and Florey HW. Changes in the endothelium of the aorta and the behaviour of macrophages in experimental atheroma of rabbits. J Pathol Bacteriol 75: 245-251, 1958.

548. Potteaux S, Esposito B, van Oostrom O, Brun V, Ardouin P, Groux H, Tedgui A, and Mallat Z. Leukocyte-derived interleukin 10 is required for protection against atherosclerosis in low-density lipoprotein receptor knockout mice. Arterioscler Thromb Vasc Biol 24: 1474-1478, 2004.

549. Potter PK, Cortes-Hernandez J, Quartier P, Botto M, and Walport MJ. Lupus-prone mice have an abnormal response to thioglycolate and an impaired clearance of apoptotic cells. $J$ Immunol 170: 3223-3232, 2003.

550. Prakken BJ, Samodal R, Le TD, Giannoni F, Yung GP, Scavulli J, Amox D, Roord S, de Kleer I, Bonnin D, Lanza P, Berry C, Massa M, Billetta R, and Albani S. Epitope-specific immunotherapy induces immune deviation of proinflammatory $\mathrm{T}$ cells in rheumatoid arthritis. Proc Natl Acad Sci USA 101: 4228-4233, 2004.

551. Prasad KS, Andre P, Yan Y, and Phillips DR. The platelet CD40L/GP IIb-IIIa axis in atherothrombotic disease. Curr Opin Hematol 10: 356-361, 2003.

552. Proudfoot JM, Croft KD, Puddey IB, and Beilin LJ. Angiotensin II type 1 receptor antagonists inhibit basal as well as lowdensity lipoprotein and platelet-activating factor-stimulated human monocyte chemoattractant protein-1. J Pharmacol Exp Ther 305: $846-853,2003$.

553. Pueyo ME, Gonzalez W, Nicoletti A, Savoie F, Arnal JF, and Michel JB. Angiotensin II stimulates endothelial vascular cell adhesion molecule-1 via nuclear factor-kappaB activation induced by intracellular oxidative stress. Arterioscler Thromb Vasc Biol 20: 645-651, 2000

554. Qu C, Edwards EW, Tacke F, Angeli V, Llodra J, SanchezSchmitz G, Garin A, Haque NS, Peters W, van Rooijen N, Sanchez-Torres C, Bromberg J, Charo IF, Jung S, Lira SA, and Randolph GJ. Role of CCR8 and other chemokine pathways in the migration of monocyte-derived dendritic cells to lymph nodes. J Exp Med 200: 1231-1241, 2004.

555. Quinn MT, Parthasarathy S, Fong LG, and Steinberg D. Oxidatively modified low density lipoproteins: a potential role in recruitment and retention of monocyte/macrophages during atherogenesis. Proc Natl Acad Sci USA 84: 2995-2998, 1987.

556. Quinn MT, Parthasarathy S, and Steinberg D. Endothelial cellderived chemotactic activity for mouse peritoneal macrophages and the effects of modified forms of low density lipoprotein. Proc Natl Acad Sci USA 82: 5949-5953, 1985. 
557. Rahman ST, Lauten WB, Khan QA, Navalkar S, Parthasarathy S, and Khan BV. Effects of eprosartan versus hydrochlorothiazide on markers of vascular oxidation and inflammation and blood pressure (renin-angiotensin system antagonists, oxidation, and inflammation). Am J Cardiol 89: 686-690, 2002.

558. Rallidis LS, Zolindaki MG, Pentzeridis PC, Poulopoulos KP, Velissaridou AH, and Apostolou TS. Raised concentrations of macrophage colony stimulating factor in severe unstable angina beyond the acute phase are strongly predictive of long term outcome. Heart 90: 25-29, 2004.

559. Randle JC, Harding MW, Ku G, Schonharting M, and Kurrle R. ICE/caspase-1 inhibitors as novel anti-inflammatory drugs. Exp Opin Invest Drugs 10: 1207-1209, 2001.

560. Rayner K, Van Eersel S, Groot PH, and Reape TJ. Localisation of mRNA for JE/MCP-1 and its receptor CCR2 in atherosclerotic lesions of the ApoE knockout mouse. J Vasc Res 37: 93-102, 2000.

561. Raz I, Elias D, Avron A, Tamir M, Metzger M, and Cohen IR. Beta-cell function in new-onset type 1 diabetes and immunomodulation with a heat-shock protein peptide (DiaPep277): a randomised, double-blind, phase II trial. Lancet 358: 1749-1753, 2001.

562. Razin E and Marx G. Thrombin-induced degranulation of cultured bone marrow-derived mast cells. J Immunol 133: 3282-3285, 1984.

563. Reardon CA, Blachowicz L, White T, Cabana V, Wang Y, Lukens J, Bluestone J, and Getz GS. Effect of immune deficiency on lipoproteins and atherosclerosis in male apolipoprotein E-deficient mice. Arterioscler Thromb Vasc Biol 21: 1011-1016, 2001.

564. Reddick RL, Zhang SH, and Maeda N. Atherosclerosis in mice lacking apo E. Evaluation of lesional development and progression. Arterioscler Thromb 14: 141-147, 1994.

565. Reddy ST, Grijalva V, Ng C, Hassan K, Hama S, Mottahedeh R, Wadleigh DJ, Navab M, and Fogelman AM. Identification of genes induced by oxidized phospholipids in human aortic endothelial cells. Vasc Pharmacol 38: 211-218, 2002.

566. Reifenberg K, Lehr HA, Baskal D, Wiese E, Schaefer SC, Black S, Samols D, Torzewski M, Lackner KJ, Husmann M, Blettner M, and Bhakdi S. Role of C-reactive protein in atherogenesis: can the apolipoprotein $\mathrm{E}$ knockout mouse provide the answer? Arterioscler Thromb Vasc Biol 25: 1641-1646, 2005.

567. Reilly MP, Lehrke M, Wolfe ML, Rohatgi A, Lazar MA, and Rader DJ. Resistin is an inflammatory marker of atherosclerosis in humans. Circulation 111: 932-939, 2005.

568. Ricci R, Sumara G, Sumara I, Rozenberg I, Kurrer M, Akhme$\operatorname{dov}$ A, Hersberger M, Eriksson U, Eberli FR, Becher B, Boren J, Chen M, Cybulsky MI, Moore KJ, Freeman MW, Wagner EF, Matter CM, and Luscher TF. Requirement of JNK2 for scavenger receptor A-mediated foam cell formation in atherogenesis. Science 306: 1558-1561, 2004.

569. Ridker PM, Cannon CP, Morrow D, Rifai N, Rose LM, McCabe CH, Pfeffer MA, and Braunwald E. C-reactive protein levels and outcomes after statin therapy. N Engl J Med 352: 20-28, 2005.

570. Ridker PM, Rifai N, Stampfer MJ, and Hennekens CH. Plasma concentration of interleukin- 6 and the risk of future myocardial infarction among apparently healthy men. Circulation 101: 1767$1772,2000$.

571. Rifkin DB. Latent transforming growth factor-beta (TGF-beta) binding proteins: orchestrators of TGF-beta availability. $J$ Biol Chem 280: 7409-7412, 2005.

572. Robertson AK, Rudling M, Zhou X, Gorelik L, Flavell RA, and Hansson GK. Disruption of TGF-beta signaling in T cells accelerates atherosclerosis. J Clin Invest 112: 1342-1350, 2003.

573. Rollins BJ and Pober JS. Interleukin-4 induces the synthesis and secretion of mcp-1/je by human endothelial cells. Am J Pathol 138: 1315-1319, 1991.

574. Romuk E, Skrzep-Poloczek B, Wojciechowska C, Tomasik A, Birkner E, Wodniecki J, Gabrylewicz B, Ochala A, and Tendera M. Selectin-P and interleukin-8 plasma levels in coronary heart disease patients. Eur J Clin Invest 32: 657-661, 2002.

575. Rong JX, Berman JW, Taubman MB, and Fisher EA. Lysophosphatidylcholine stimulates monocyte chemoattractant protein-1 gene expression in rat aortic smooth muscle cells. Arterioscler Thromb Vasc Biol 22: 1617-1623, 2002.
576. Rosenfeld ME and Ross R. Macrophage and smooth muscle cell proliferation in atherosclerotic lesions of WHHL and comparably hypercholesterolemic fat-fed rabbits. Arteriosclerosis 10: 680-687, 1990.

577. Rosenfeld ME, Ylaherttuala S, Lipton BA, Ord VA, Witztum JL, and Steinberg D. Macrophage colony-stimulating factor messenger RNA and protein in atherosclerotic lesions of rabbits and humans. Am J Pathol 140: 291-300, 1992.

578. Ross R. Atherosclerosis-an inflammatory disease. $N$ Engl J Med 340: 115-126, 1999.

579. Ross R. The biology of platelet-derived growth factor. Cell 46: 155-169, 1986.

580. Ross R and Glomset JA. The pathogenesis of atherosclerosis. N Engl J Med 295: 369-377; 420-425, 1976.

581. Ross R, Masuda J, Raines EW, Gown AM, Katsuda S, Sasahara M, Malden LT, Masuko H, and Sato H. Localization of PDGF-B protein in macrophages in all phases of atherogenesis. Science 248: 1009-1012, 1990

582. Rossi A, Kapahi P, Natoli G, Takahashi T, Chen Y, Karin M, and Santoro MG. Anti-inflammatory cyclopentenone prostaglandins are direct inhibitors of IkappaB kinase. Nature 403: 103-108, 2000.

583. Ruffer M. On arterial lesions found in Egyptian mummies (158 BC-AD 525). J Pathol Bact 15: 453-462, 1911.

584. Ruiz-Ortega M, Bustos C, Hernandez-Presa MA, Lorenzo O, Plaza JJ, and Egido J. Angiotensin II participates in mononuclear cell recruitment in experimental immune complex nephritis through nuclear factor-kappa B activation and monocyte chemoattractant protein-1 synthesis. J Immunol 161: 430-439, 1998.

585. Rus HG, Niculescu F, and Vlaicu R. Tumor necrosis factor-alpha in human arterial wall with atherosclerosis. Atherosclerosis 89: 247-254, 1991.

586. Rus HG, Vlaicu R, and Niculescu F. Interleukin-6 and interleukin-8 protein and gene expression in human arterial atherosclerotic wall. Atherosclerosis 127: 263-271, 1996.

587. Rutledge BJ, Rayburn H, Rosenberg R, North RJ, Gladue RP, Corless CL, and Rollins BJ. High level monocyte chemoattractant protein-1 expression in transgenic mice increases their susceptibility to intracellular pathogens. J Immunol 155: 4838-4843, 1995.

588. Sabapathy K, Hu Y, Kallunki T, Schreiber M, David JP, Jochum W, Wagner EF, and Karin M. JNK2 is required for efficient T-cell activation and apoptosis but not for normal lymphocyte development. Curr Biol 9: 116-125, 1999.

589. Saikku P, Leinonen M, Mattila K, Ekman MR, Nieminen MS, Makela PH, Huttunen JK, and Valtonen V. Serological evidence of an association of a novel Chlamydia, TWAR, with chronic coronary heart disease and acute myocardial infarction. Lancet 2: 983-986, 1988.

590. Saitoh T, Kishida H, Tsukada Y, Fukuma Y, Sano J, Yasutake M, Fukuma N, Kusama Y, and Hayakawa H. Clinical significance of increased plasma concentration of macrophage colony-stimulating factor in patients with angina pectoris. $J$ Am Coll Cardiol 35: $655-665,2000$.

591. Salomon B, Lenschow DJ, Rhee L, Ashourian N, Singh B, Sharpe A, and Bluestone JA. B7/CD28 costimulation is essential for the homeostasis of the CD4+CD25+ immunoregulatory T cells that control autoimmune diabetes. Immunity 12: 431-440, 2000.

592. Sambrano GR and Steinberg D. Recognition of oxidatively damaged and apoptotic cells by an oxidized low density lipoprotein receptor on mouse peritoneal macrophages: role of membrane phosphatidylserine. Proc Natl Acad Sci USA 92: 1396-1400, 1995.

593. Sandison A. Degenerative vascular disease in the Egyptian mummy. Med Hist 6: 77-81, 1962.

594. Sano S, Itami S, Takeda K, Tarutani M, Yamaguchi Y, Miura H, Yoshikawa K, Akira S, and Takeda J. Keratinocyte-specific ablation of Stat3 exhibits impaired skin remodeling, but does not affect skin morphogenesis. EMBO J 18: 4657-4668, 1999.

595. Sauter B, Albert ML, Francisco L, Larsson M, Somersan S, and Bhardwaj $\mathbf{N}$. Consequences of cell death: exposure to necrotic tumor cells, but not primary tissue cells or apoptotic cells, induces the maturation of immunostimulatory dendritic cells. $J$ Exp Med 191: 423-434, 2000. 
596. Savill J, Dransfield I, Gregory C, and Haslett C. A blast from the past: clearance of apoptotic cells regulates immune responses. Nat Rev Immunol 2: 965-975, 2002.

597. Scarpati EM and Sadler JE. Regulation of endothelial cell coagulant properties. Modulation of tissue factor, plasminogen activator inhibitors, and thrombomodulin by phorbol 12-myristate 13acetate and tumor necrosis factor. J Biol Chem 264: 20705-20713, 1989.

598. Schieffer B, Selle T, Hilfiker A, Hilfiker-Kleiner D, Grote K, Tietge UJ, Trautwein C, Luchtefeld M, Schmittkamp C, Heeneman S, Daemen MJ, and Drexler H. Impact of interleukin-6 on plaque development and morphology in experimental atherosclerosis. Circulation 110: 3493-3500, 2004.

599. Schiopu A, Bengtsson J, Soderberg I, Janciauskiene S, Lindgren S, Ares MP, Shah PK, Carlsson R, Nilsson J, and Fredrikson GN. Recombinant human antibodies against aldehydemodified apolipoprotein B-100 peptide sequences inhibit atherosclerosis. Circulation 110: 2047-2052, 2004.

600. Schleicher U, Hesse A, and Bogdan C. Minute numbers of contaminant CD8 $+\mathrm{T}$ cells or CD11b $+\mathrm{CD} 11 \mathrm{c}+\mathrm{NK}$ cells are the source of IFN-gamma in IL-12/IL-18-stimulated mouse macrophage populations. Blood 105: 1319-1328, 2005.

601. Schleimer RP, Sterbinsky SA, Kaiser J, Bickel CA, Klunk DA, Tomioka K, Newman W, Luscinskas FW, Gimbrone MA Jr, McIntyre BW, and Bochner BS. IL-4 induces adherence of human eosinophils and basophils but not neutrophils to endothelium Association with expression of VCAM-1. J Immunol 148: 1086-1092, 1992.

602. Schnyder B, Lugli S, Feng N, Etter H, Lutz RA, Ryffel B, Sugamura K, Wunderli-Allenspach $\mathbf{H}$, and Moser R. Interleukin-4 (IL-4) and IL-13 bind to a shared heterodimeric complex on endothelial cells mediating vascular cell adhesion molecule-1 induction in the absence of the common gamma chain. Blood 87: 4286-4295, 1996.

603. Schober A, Bernhagen J, Thiele M, Zeiffer U, Knarren S, Roller M, Bucala R, and Weber C. Stabilization of atherosclerotic plaques by blockade of macrophage migration inhibitory factor after vascular injury in apolipoprotein E-deficient mice. Circulation 109: 380-385, 2004.

604. Schober A, Manka D, von Hundelshausen P, Huo Y, Hanrath P, Sarembock IJ, Ley K, and Weber C. Deposition of platelet RANTES triggering monocyte recruitment requires P-selectin and is involved in neointima formation after arterial injury. Circulation 106: 1523-1529, 2002.

605. Schonbeck U and Libby P. CD40 signaling and plaque instability. Circ Res 89: 1092-1103, 2001.

606. Schonbeck U, Mach F, and Libby P. Generation of biologically active IL-1beta by matrix metalloproteinases: a novel caspase-1independent pathway of IL-1beta processing. J Immunol 161: 3340-3346, 1998

607. Schonbeck U, Sukhova GK, Gerdes N, and Libby P. T(H)2 predominant immune responses prevail in human abdominal aortic aneurysm. Am J Pathol 161: 499-506, 2002.

608. Schonbeck U, Varo N, Libby P, Buring J, and Ridker PM. Soluble CD40L and cardiovascular risk in women. Circulation 104: 2266-2268, 2001.

609. Schoppet M, Preissner KT, and Hofbauer LC. RANK ligand and osteoprotegerin: paracrine regulators of bone metabolism and vascular function. Arterioscler Thromb Vasc Biol 22: 549-553, 2002.

610. Schorle H, Holtschke T, Hunig T, Schimpl A, and Horak I. Development and function of T cells in mice rendered interleukin-2 deficient by gene targeting. Nature 352: 621-624, 1991.

611. Schrader JW, Moyer C, Ziltener HJ, and Reinisch CL. Release of the cytokines colony-stimulating factor-1, granulocyte-macrophage colony-stimulating factor, and IL- 6 by cloned murine vascular smooth muscle cells. J Immunol 146: 3799-3808, 1991.

612. Schreck R and Baeuerle PA. A role for oxygen radicals as second messengers. Trends Cell Biol 1: 39-42, 1991.

613. Schreyer SA, Peschon JJ, and Leboeuf RC. Accelerated atherosclerosis in mice lacking tumor necrosis factor receptor p55. J Biol Chem 271: 26174-26178, 1996.
614. Schreyer SA, Vick CM, and LeBoeuf RC. Loss of lymphotoxinalpha but not tumor necrosis factor-alpha reduces atherosclerosis in mice. J Biol Chem 277: 12364-12368, 2002.

615. Schrijvers DM, De Meyer GR, Kockx MM, Herman AG, and Martinet W. Phagocytosis of apoptotic cells by macrophages is impaired in atherosclerosis. Arterioscler Thromb Vasc Biol 25: 1256-1261, 2005.

616. Schwedler SB, Amann K, Wernicke K, Krebs A, Nauck M, Wanner C, Potempa LA, and Galle J. Native C-reactive protein increases whereas modified C-reactive protein reduces atherosclerosis in apolipoprotein E-knockout mice. Circulation 112: 1016$1023,2005$.

617. Scott RS, McMahon EJ, Pop SM, Reap EA, Caricchio R, Cohen PL, Earp HS, and Matsushima GK. Phagocytosis and clearance of apoptotic cells is mediated by MER. Nature 411: 207-211, 2001.

618. Seino Y, Ikeda U, Ikeda M, Hasegawa T, Misawa Y, Yamamoto $\mathbf{K}$, Kano S, and Shimada K. Interleukin 6 gene transcripts are expressed in human atherosclerotic lesions. Cytokine 6: 87-91, 1994.

619. Seki Y, Inoue H, Nagata N, Hayashi K, Fukuyama S, Matsumoto K, Komine O, Hamano S, Himeno K, Inagaki-Ohara K, Cacalano N, O'Garra A, Oshida T, Saito H, Johnston JA, Yoshimura A, and Kubo M. SOCS-3 regulates onset and maintenance of T(H)2-mediated allergic responses. Nat Med 9: 1047-1054, 2003.

620. SenBanerjee S, Lin Z, Atkins GB, Greif DM, Rao RM, Kumar A, Feinberg MW, Chen Z, Simon DI, Luscinskas FW, Michel TM, Gimbrone MA Jr, Garcia-Cardena G, and Jain MK. KLF2 is a novel transcriptional regulator of endothelial proinflammatory activation. J Exp Med 199: 1305-1315, 2004.

621. Sendobry SM, Cornicelli JA, Welch K, Grusby MJ, and Daugherty A. Absence of T lymphocyte-derived cytokines fails to diminish macrophage 12/15-lipoxygenase expression in vivo. J Immunol 161: 1477-1482, 1998.

622. Seta Y, Kanda T, Tanaka T, Arai M, Sekiguchi K, Yokoyama T, Kurimoto M, Tamura J, and Kurabayashi M. Interleukin 18 in acute myocardial infarction. Heart 84: 668, 2000.

623. Setoguchi R, Hori S, Takahashi T, and Sakaguchi S. Homeostatic maintenance of natural Foxp3(+) CD25(+) CD4(+) regulatory $\mathrm{T}$ cells by interleukin (IL)-2 and induction of autoimmune disease by IL-2 neutralization. J Exp Med 201: 723-735, 2005.

624. Shah PK, Chyu KY, Fredrikson GN, and Nilsson J. Vaccination for atherosclerosis: a novel therapeutic paradigm. Expert Rev Vaccines 3: 711-716, 2004.

625. Shao J, Nangaku M, Miyata T, Inagi R, Yamada K, Kurokawa $\mathbf{K}$, and Fujita T. Imbalance of T-cell subsets in angiotensin IIinfused hypertensive rats with kidney injury. Hypertension 42 : 31-38, 2003.

626. Shaulian $\mathbf{E}$ and Karin M. AP-1 as a regulator of cell life and death. Nat Cell Biol 4: E131-E136, 2002.

627. Shaw PX, Horkko S, Chang MK, Curtiss LK, Palinski W, Silverman GJ, and Witztum JL. Natural antibodies with the T15 idiotype may act in atherosclerosis, apoptotic clearance, and protective immunity. J Clin Invest 105: 1731-1740, 2000.

628. Shi W, Haberland ME, Jien ML, Shih DM, and Lusis AJ. Endothelial responses to oxidized lipoproteins determine genetic susceptibility to atherosclerosis in mice. Circulation 102: 75-81, 2000.

629. Shi W, Wang NJ, Shih DM, Sun VZ, Wang X, and Lusis AJ. Determinants of atherosclerosis susceptibility in the C3H and C57BL/6 mouse model: evidence for involvement of endothelial cells but not blood cells or cholesterol metabolism. Circ Res 86: 1078-1084, 2000.

630. Shimaoka T, Kume N, Minami M, Hayashida K, Kataoka H, Kita T, and Yonehara S. Molecular cloning of a novel scavenger receptor for oxidized low density lipoprotein, SR-PSOX, on macrophages. J Biol Chem 275: 40663-40666, 2000.

631. Shimaoka T, Nakayama T, Hieshima K, Kume N, Fukumoto N, Minami M, Hayashida K, Kita T, Yoshie O, and Yonehara S. Chemokines generally exhibit scavenger receptor activity through their receptor-binding domain. J Biol Chem 279: 26807-26810, 2004. 
632. Shimizu K, Shichiri M, Libby $\mathbf{P}$, Lee RT, and Mitchell RN. Th2-predominant inflammation and blockade of IFN-gamma signaling induce aneurysms in allografted aortas. $J$ Clin Invest 114: 300-308, 2004.

633. Shimizu T, Nishihira J, Watanabe H, Abe R, Honda A, Ishibashi T, and Shimizu $H$. Macrophage migration inhibitory factor is induced by thrombin and factor Xa in endothelial cells. $J$ Biol Chem 279: 13729-13737, 2004.

634. Shimoda K, van Deursen J, Sangster MY, Sarawar SR, Carson RT, Tripp RA, Chu C, Quelle FW, Nosaka T, Vignali DA, Doherty PC, Grosveld G, Paul WE, and Ihle JN. Lack of IL-4induced Th2 response and IgE class switching in mice with disrupted Stat6 gene. Nature 380: 630-633, 1996.

635. Shouda T, Yoshida T, Hanada T, Wakioka T, Oishi M, Miyoshi K, Komiya S, Kosai K, Hanakawa Y, Hashimoto K, Nagata K, and Yoshimura A. Induction of the cytokine signal regulator SOCS3/CIS3 as a therapeutic strategy for treating inflammatory arthritis. J Clin Invest 108: 1781-1788, 2001.

636. Shull MM, Ormsby I, Kier AB, Pawlowski S, Diebold RJ, Yin M, Allen R, Sidman C, Proetzel G, Calvin D, Annunziata N, and Doetschman T. Targeted disruption of the mouse transforming growth factor- $\beta 1$ gene results in multifocal inflammatory disease. Nature 359: 693-699, 1992.

637. Silence J, Lupu F, Collen D, and Lijnen HR. Persistence of atherosclerotic plaque but reduced aneurysm formation in mice with stromelysin-1 (MMP-3) gene inactivation. Arterioscler Thromb Vasc Biol 21: 1440-1445, 2001.

638. Simon AD, Yazdani S, Wang W, Schwartz A, and Rabbani LE. Elevated plasma levels of interleukin-2 and soluble IL-2 receptor in ischemic heart disease. Clin Cardiol 24: 253-256, 2001.

639. Simonet WS, Hughes TM, Nguyen HQ, Trebasky LD, Danilenko DM, and Medlock ES. Long-term impaired neutrophil migration in mice overexpressing human interleukin-8. J Clin Invest 94: 1310-1319, 1994.

640. Singer II, Scott S, Chin J, Bayne EK, Limjuco G, Weidner J, Miller DK, Chapman K, and Kostura MJ. The interleukin-1 beta-converting enzyme (ice) is localized on the external cell surface membranes and in the cytoplasmic ground substance of human monocytes by immune-electron microscopy. J Exp Med 182: 1447-1459, 1995.

641. Skoog T, Dichtl W, Boquist S, Skoglund-Andersson C, Karpe F, Tang R, Bond MG, de Faire U, Nilsson J, Eriksson P, and Hamsten A. Plasma tumour necrosis factor-alpha and early carotid atherosclerosis in healthy middle-aged men. Eur Heart $J$ 23: 376-383, 2002.

642. Smiley ST, King JA, and Hancock WW. Fibrinogen stimulates macrophage chemokine secretion through toll-like receptor 4 . J Immunol 167: 2887-2894, 2001.

643. Smith JD, Trogan E, Ginsberg M, Grigaux C, Tian J, and Miyata M. Decreased atherosclerosis in mice deficient in both macrophage colony-stimulating factor (op) and apolipoprotein E. Proc Natl Acad Sci USA 92: 8264-8268, 1995.

644. Smook ML, Heeringa P, Damoiseaux JG, Daemen MJ, de Winther MP, Gijbels MJ, Beckers L, Lutgens E, and Cohen Tervaert JW. Leukocyte CD40L deficiency affects the CD25(+) CD4 T cell population but does not affect atherosclerosis. Atherosclerosis 183: 275-282, 2005.

645. Sohn JH, Bora PS, Suk HJ, Molina H, Kaplan HJ, and Bora NS. Tolerance is dependent on complement C3 fragment iC3b binding to antigen-presenting cells. Nat Med 9: 206-212, 2003.

646. Song $\mathbf{L}$, Leung $\mathbf{C}$, and Schindler C. Lymphocytes are important in early atherosclerosis. J Clin Invest 108: 251-259, 2001.

647. Stamler J, Wentworth D, and Neaton JD. Is relationship between serum cholesterol and risk of premature death from coronary heart disease continuous and graded? Findings in 356,222 primary screenees of the Multiple Risk Factor Intervention Trial (MRFIT). JAMA 256: 2823-2828, 1986.

648. Stary HC, Chandler AB, Dinsmore RE, Fuster V, Glagov S, Insull W, Rosenfeld ME, Schwartz CJ, Wagner WD, and Wissler RW. A definition of advanced types of atherosclerotic lesions and a histological classification of atherosclerosis: a report from the committee on vascular lesions of the council on arterio- sclerosis, American Heart Association. Circulation 92: 1355-1374, 1995.

649. Stary HC, Chandler AB, Glagov S, Guyton JR, Insull W, Rosenfeld ME, Schaffer SA, Schwartz CJ, Wagner WD, and Wissler RW. A definition of initial, fatty streak, and intermediate lesions of atherosclerosis: a report from the committee on vascular lesions of the council on arteriosclerosis, American Heart Association. Arterioscler Thromb 14: 840-856, 1994.

650. Steinberg D, Parthasarathy S, Carew TE, Khoo JC, and Witztum JL. Beyond cholesterol. Modifications of low-density lipoprotein that increase its atherogenicity. $N$ Engl J Med 320: 915-924, 1989.

651. Steinman RM, Turley S, Mellman I, and Inaba K. The induction of tolerance by dendritic cells that have captured apoptotic cells. $J$ Exp Med 191: 411-416, 2000.

652. Stemme S, Faber B, Holm J, Wiklund O, Witztum JL, and Hansson GK. T lymphocytes from human atherosclerotic plaques recognize oxidized low density lipoprotein. Proc Natl Acad Sci USA 92: 3893-3897, 1995.

653. Subbanagounder G, Wong JW, Lee H, Faull KF, Miller E, Witztum JL, and Berliner JA. Epoxyisoprostane and epoxycyclopentenone phospholipids regulate monocyte chemotactic protein- 1 and interleukin- 8 synthesis. Formation of these oxidized phospholipids in response to interleukin-1beta. J Biol Chem 277: 7271-7281, 2002.

654. Sundstedt A, O'Neill EJ, Nicolson KS, and Wraith DC. Role for IL-10 in suppression mediated by peptide-induced regulatory $\mathrm{T}$ cells in vivo. J Immunol 170: 1240-1248, 2003.

655. Suri-Payer E and Cantor H. Differential cytokine requirements for regulation of autoimmune gastritis and colitis by CD4(+)CD25(+) T cells. J Autoimmunity 16: 115-123, 2001.

656. Sutherland MR, Raynor CM, Leenknegt H, Wright JF, and Pryzdial EL. Coagulation initiated on herpesviruses. Proc Natl Acad Sci USA 94: 13510-13514, 1997.

657. Suzuki A, Hanada T, Mitsuyama K, Yoshida T, Kamizono S, Hoshino T, Kubo M, Yamashita A, Okabe M, Takeda K, Akira S, Matsumoto S, Toyonaga A, Sata $M$, and Yoshimura A. CIS3/SOCS3/SSI3 plays a negative regulatory role in STAT3 activation and intestinal inflammation. J Exp Med 193: 471-481, 2001.

658. Suzuki J, Iwai M, Nakagami H, Wu L, Chen R, Sugaya T, Hamada M, Hiwada K, and Horiuchi M. Role of angiotensin II-regulated apoptosis through distinct AT1 and AT2 receptors in neointimal formation. Circulation 106: 847-853, 2002.

659. Swafford AN Jr, Bratz IN, Knudson JD, Rogers PA, Timmerman JM, Tune JD, and Dick GM. C-reactive protein does not relax vascular smooth muscle: effects mediated by sodium azide in commercially available preparations. Am J Physiol Heart Circ Physiol 288: H1786-H1795, 2005.

660. Szabo SJ, Kim ST, Costa GL, Zhang X, Fathman CG, and Glimcher LH. A novel transcription factor, T-bet, directs Th1 lineage commitment. Cell 100: 655-669, 2000.

661. Tager AM, Bromley SK, Medoff BD, Islam SA, Bercury SD, Friedrich EB, Carafone AD, Gerszten RE, and Luster AD. Leukotriene $\mathrm{B}_{4}$ receptor BLT1 mediates early effector T cell recruitment. Nat Immunol 4: 982-990, 2003.

662. Takabe W, Kanai Y, Chairoungdua A, Shibata N, Toi S, Kobayashi M, Kodama T, and Noguchi N. Lysophosphatidylcholine enhances cytokine production of endothelial cells via induction of L-type amino acid transporter 1 and cell surface antigen $4 \mathrm{~F} 2$. Arterioscler Thromb Vasc Biol 24: 1640-1645, 2004.

663. Takahara N, Kashiwagi A, Maegawa H, and Shigeta Y. Lysophosphatidylcholine stimulates the expression and production of MCP-1 by human vascular endothelial cells. Metabolism 45: $559-$ $564,1996$.

664. Takeda K, Clausen BE, Kaisho T, Tsujimura T, Terada N, Forster I, and Akira S. Enhanced Th1 activity and development of chronic enterocolitis in mice devoid of Stat 3 in macrophages and neutrophils. Immunity 10: 39-49, 1999.

665. Takeda K, Kaisho T, and Akira S. Toll-like receptors. Annu Rev Immunol 21: 335-376, 2003.

666. Takeda K, Kaisho T, Yoshida N, Takeda J, Kishimoto T, and Akira S. Stat3 activation is responsible for IL-6-dependent T cell proliferation through preventing apoptosis: generation and charac- 
terization of T cell-specific Stat3-deficient mice. J Immunol 161: 4652-4660, 1998.

667. Talanian RV, Quinlan C, Trautz S, Hackett MC, Mankovich JA, Banach D, Ghayur T, Brady KD, and Wong WW. Substrate specificities of caspase family proteases. J Biol Chem 272: 96779682, 1997.

668. Taylor PR, Carugati A, Fadok VA, Cook HT, Andrews M, Carroll MC, Savill JS, Henson PM, Botto M, and Walport MJ. A hierarchical role for classical pathway complement proteins in the clearance of apoptotic cells in vivo. J Exp Med 192: 359-366, 2000.

669. Tedgui A and Mallat Z. Anti-inflammatory mechanisms in the vascular wall. Circ Res 88: 877-887, 2001.

670. Tellides G, Tereb DA, Kirkiles-Smith NC, Kim RW, Wilson JH, Schechner JS, Lorber MI, and Pober JS. Interferon-gamma elicits arteriosclerosis in the absence of leukocytes. Nature 403: 207-211, 2000.

671. Tenger C, Sundborger A, Jawien J, and Zhou X. IL-18 accelerates atherosclerosis accompanied by elevation of IFN-gamma and CXCL16 expression independently of T cells. Arterioscler Thromb Vasc Biol 25: 791-796, 2005.

673. Termeer C, Benedix F, Sleeman J, Fieber C, Voith U, Ahrens T, Miyake K, Freudenberg M, Galanos C, and Simon JC. Oligosaccharides of hyaluronan activate dendritic cells via toll-like receptor 4. J Exp Med 195: 99-111, 2002.

674. Terry CM, Clikeman JA, Hoidal JR, and Callahan KS. Effect of tumor necrosis factor- $\alpha$ and interleukin- $1 \alpha$ on heme oxygenase-1 expression in human endothelial cells. Am J Physiol Heart Circ Physiol 274: H883-H891, 1998.

675. Tilg H, Trehu E, Atkins MB, Dinarello CA, and Mier JW. Interleukin-6 (IL-6) as an anti-inflammatory cytokine: induction of circulating IL-1 receptor antagonist and soluble tumor necrosis factor receptor p55. Blood 83: 113-118, 1994.

676. Tipping PG and Hancock WW. Production of tumor necrosis factor and interleukin-1 by macrophages from human atheromatous plaques. Am J Pathol 142: 1721-1728, 1993.

677. Tournier C, Dong C, Turner TK, Jones SN, Flavell RA, and Davis RJ. MKK7 is an essential component of the JNK signal transduction pathway activated by proinflammatory cytokines. Genes Dev 15: 1419-1426, 2001.

678. Tournier C, Hess P, Yang DD, Xu J, Turner TK, Nimnual A, Bar-Sagi D, Jones SN, Flavell RA, and Davis RJ. Requirement of JNK for stress-induced activation of the cytochrome $c$-mediated death pathway. Science 288: 870-874, 2000.

679. Tricot $\mathbf{O}$, Mallat Z, Lesèche G, and Tedgui A. Distribution of apoptosis in human carotid atherosclerosis. Relation to flow direction. Communication at the American Heart Association Meeting, Atlanta GA, 1999.

680. Tsan MF and Gao B. Cytokine function of heat shock proteins. Am J Physiol Cell Physiol 286: C739-C744, 2004.

681. Tschopp J, Martinon F, and Burns K. NALPs: a novel protein family involved in inflammation. Nat Rev Mol Cell Biol 4: 95-104, 2003.

682. Tummala PE, Chen XL, Sundell CL, Laursen JB, Hammes CP, Alexander RW, Harrison DG, and Medford RM. Angiotensin II induces vascular cell adhesion molecule-1 expression in rat vasculature: a potential link between the renin-angiotensin system and atherosclerosis. Circulation 100: 1223-1229, 1999

683. Tupin E, Nicoletti A, Elhage R, Rudling M, Ljunggren HG, Hansson GK, and Berne GP. CD1d-dependent activation of NKT cells aggravates atherosclerosis. J Exp Med 199: 417-422, 2004.

684. Ulloa L, Doody J, and Massague J. Inhibition of transforming growth factor-beta/SMAD signalling by the interferon-gamma/STAT pathway. Nature 397: 710-713, 1999.

685. Upadhya S, Mooteri S, Peckham N, and Pai RG. Atherogenic effect of interleukin-2 and antiatherogenic effect of interleukin-2 antibody in apo-E-deficient mice. Angiology 55: 289-294, 2004.

686. Urbich $\mathbf{C}$ and Dimmeler S. Endothelial progenitor cells: characterization and role in vascular biology. Circ Res 95: 343-353, 2004.

687. Uyemura K, Demer LL, Castle SC, Jullien D, Berliner JA, Gately MK, Warrier RR, Pham N, Fogelman AM, and Modlin RL. Cross-regulatory roles of interleukin (IL)-12 and IL-10 in atherosclerosis. J Clin Invest 97: 2130-2138, 1996.
688. Vachharajani TJ, Work J, Issekutz AC, and Granger DN. Heme oxygenase modulates selectin expression in different regional vascular beds. Am J Physiol Heart Circ Physiol 278: H1613-H1617, 2000.

689. Van den Berg CW, Taylor KE, and Lang D. C-reactive proteininduced in vitro vasorelaxation is an artefact caused by the presence of sodium azide in commercial preparations. Arterioscler Thromb Vasc Biol 24: e168-171, 2004.

690. Van der Wal AC, Becker AE, van der Loos CM, and Das PK. Site of intimal rupture or erosion of thrombosed coronary atherosclerotic plaques is characterized by an inflammatory process irrespective of the dominant plaque morphology. Circulation 89: $36-$ 44, 1994.

691. Vandivier RW, Fadok VA, Hoffmann PR, Bratton DL, Penvari C, Brown KK, Brain JD, Accurso FJ, and Henson PM. Elastasemediated phosphatidylserine receptor cleavage impairs apoptotic cell clearance in cystic fibrosis and bronchiectasis. J Clin Invest 109: 661-670, 2002.

692. Van Lenten BJ and Fogelman AM. Lipopolysaccharide-induced inhibition of scavenger receptor expression in human monocytemacrophages is mediated through tumor necrosis factor- $\alpha$. J Immunol 148: 112-116, 1992.

693. Varo N, de Lemos JA, Libby P, Morrow DA, Murphy SA, Nuzzo R, Gibson CM, Cannon CP, Braunwald E, and Schonbeck U. Soluble CD40L: risk prediction after acute coronary syndromes. Circulation 108: 1049-1052, 2003.

694. Vaughan CJ and Gotto AM Jr. Update on statins: 2003. Circulation 110: 886-892, 2004.

695. Veillard NR, Kwak B, Pelli G, Mulhaupt F, James RW, Proudfoot AE, and Mach F. Antagonism of RANTES receptors reduces atherosclerotic plaque formation in mice. Circ Res 94: 253-261, 2004.

696. Verbovetski I, Bychkov H, Trahtemberg U, Shapira I, Hareuveni M, Ben-Tal O, Kutikov I, Gill O, and Mevorach D. Opsonization of apoptotic cells by autologous iC3b facilitates clearance by immature dendritic cells, down-regulates DR and CD86, and up-regulates CC chemokine receptor 7. J Exp Med 196: 1553-1561, 2002

697. Verhamme P, Quarck R, Hao H, Knaapen M, Dymarkowski S, Bernar H, Van Cleemput J, Janssens S, Vermylen J, Gabbiani G, Kockx M, and Holvoet P. Dietary cholesterol withdrawal reduces vascular inflammation and induces coronary plaque stabilization in miniature pigs. Cardiovasc Res 56: 135-144, 2002.

698. Vieira PL, Christensen JR, Minaee S, O'Neill EJ, Barrat FJ, Boonstra A, Barthlott T, Stockinger B, Wraith DC, and O'Garra A. IL-10-secreting regulatory T cells do not express Foxp3 but have comparable regulatory function to naturally occurring CD4+CD25+ regulatory T cells. J Immunol 172: 5986-5993, 2004.

699. Vink A, Schoneveld AH, van der Meer JJ, van Middelaar BJ, Sluijter JP, Smeets MB, Quax PH, Lim SK, Borst C, Pasterkamp G, and de Kleijn DP. In vivo evidence for a role of toll-like receptor 4 in the development of intimal lesions. Circulation 106: 1985-1990, 2002.

700. Viola A and Lanzavecchia A. T cell activation determined by T cell receptor number and tunable thresholds. Science 273: 104-106, 1996.

701. Virchow R. Phlogose und Thrombose im Gefassystem. In: Gesammelte Abhanndlungen zür wissenschaftlichen Medizin. Frankfurtam-Main: F. Meidinger Sohn, 1856, p. 458-521.

702. Virella G, Atchley D, Koskinen S, Zheng D, and Lopes-Virella MF. Proatherogenic and proinflammatory properties of immune complexes prepared with purified human oxLDL antibodies and human oxLDL. Clin Immunol 105: 81-92, 2002.

703. Virmani R, Burke AP, and Farb A. Plaque rupture and plaque erosion. Thromb Haemost 82 Suppl 1: 1-3, 1999.

704. Virmani R, Kolodgie FD, Burke AP, Farb A, and Schwartz SM. Lessons from sudden coronary death: a comprehensive morphological classification scheme for atherosclerotic lesions. Arterioscler Thromb Vasc Biol 20: 1262-1275, 2000.

705. Virmani R, Kolodgie FD, Burke AP, Finn AV, Gold HK, Tulenko TN, Wrenn SP, and Narula J. Atherosclerotic plaque progression and vulnerability to rupture: angiogenesis as a source 
of intraplaque hemorrhage. Arterioscler Thromb Vasc Biol 25: 2054-2061, 2005.

706. Vlassara H, Brownlee M, Manogue KR, Dinarello CA, and Pasaglan A. Cachectin/TNF and IL-1 induced by glucose-modified proteins: role in normal tissue remodeling. Science 240: 1546-1548, 1988.

707. Von Boehmer H. Mechanisms of suppression by suppressor T cells. Nat Immunol 6: 338-344, 2005.

708. Von Der Thusen JH, Kuiper J, Fekkes ML, De Vos P, Van Berkel TJ, and Biessen EA. Attenuation of atherogenesis by systemic and local adenovirus-mediated gene transfer of interleukin-10 in LDLr-/- mice. FASEB $J$ 15: 2730-2732, 2001.

709. Von der Thusen JH, Kuiper J, van Berkel TJ, and Biessen EA. Interleukins in atherosclerosis: molecular pathways and therapeutic potential. Pharmacol Rev 55: 133-166, 2003.

710. Von Hundelshausen P, Weber KS, Huo Y, Proudfoot AE, Nelson PJ, Ley K, and Weber C. RANTES deposition by platelets triggers monocyte arrest on inflamed and atherosclerotic endothelium. Circulation 103: 1772-1777, 2001.

711. Wagner DD. New links between inflammation and thrombosis. Arterioscler Thromb Vasc Biol 25: 1321-1324, 2005.

712. Wakkach A, Cottrez F, and Groux H. Can interleukin-10 be used as a true immunoregulatory cytokine? Eur Cytokine Netw 11: 153-160, 2000.

713. Wakkach A, Fournier N, Brun V, Breittmayer JP, Cottrez F, and Groux H. Characterization of dendritic cells that induce tolerance and T regulatory 1 cell differentiation in vivo. Immunity 18 : 605-617, 2003.

714. Wallin RP, Lundqvist A, More SH, von Bonin A, Kiessling R, and Ljunggren HG. Heat-shock proteins as activators of the innate immune system. Trends Immunol 23: 130-135, 2002.

715. Walton KA, Hsieh X, Gharavi N, Wang S, Wang G, Yeh M, Cole AL, and Berliner JA. Receptors involved in the oxidized 1-palmitoyl-2-arachidonoyl-sn-glycero-3-phosphorylcholine-mediated synthesis of interleukin-8. A role for Toll-like receptor 4 and a glycosylphosphatidylinositol-anchored protein. J Biol Chem 278: 2966129666, 2003.

716. Wang C, Deng L, Hong M, Akkaraju GR, Inoue J, and Chen ZJ. TAK1 is a ubiquitin-dependent kinase of MKK and IKK. Nature 412: 346-351, 2001.

717. Wang H, Nawata J, Kakudo N, Sugimura K, Suzuki J, Sakuma M, Ikeda J, and Shirato K. The upregulation of ICAM-1 and P-selectin requires high blood pressure but not circulating reninangiotensin system in vivo. J Hypertens 22: 1323-1332, 2004.

718. Wang N, Tabas I, Winchester R, Ravalli S, Rabbani LE, and Tall A. Interleukin 8 is induced by cholesterol loading of macrophages and expressed by macrophage foam cells in human atheroma. J Biol Chem 271: 8837-8842, 1996.

719. Wang X, Ria M, Kelmenson PM, Eriksson P, Higgins DC, Samnegard A, Petros C, Rollins J, Bennet AM, Wiman B, de Faire U, Wennberg C, Olsson PG, Ishii N, Sugamura K, Hamsten A, Forsman-Semb K, Lagercrantz J, and Paigen B. Positional identification of TNFSF4, encoding OX40 ligand, as a gene that influences atherosclerosis susceptibility. Nat Genet 37: 365$372,2005$.

720. Wanidworanun $\mathbf{C}$ and Strober W. Predominant role of tumor necrosis factor-alpha in human monocyte IL-10 synthesis. J Immunol 151: 6853-6861, 1993.

721. Warner SJ, Auger KR, and Libby P. Human interleukin 1 induces interleukin 1 gene expression in human vascular smooth muscle cells. J Exp Med 165: 1316-1331, 1987.

722. Warner SJ, Auger KR, and Libby P. Interleukin 1 induces interleukin 1. II. Recombinant human interleukin 1 induces interleukin 1 production by adult human vascular endothelial cells. J Immunol 139: 1911-1917, 1987.

723. Warner SJ and Libby P. Human vascular smooth muscle cells. Target for and source of tumor necrosis factor. J Immunol 142: 100-109, 1989.

724. Wassmann S, Czech T, van Eickels M, Fleming I, Bohm M, and Nickenig G. Inhibition of diet-induced atherosclerosis and endothelial dysfunction in apolipoprotein E/angiotensin II type 1A receptor double-knockout mice. Circulation 110: 3062-3067, 2004.
725. Watanabe N, Wang YH, Lee HK, Ito T, Wang YH, Cao W, and Liu YJ. Hassall's corpuscles instruct dendritic cells to induce CD4+CD25+ regulatory $\mathrm{T}$ cells in human thymus. Nature 436: 1181-1185, 2005.

726. Watanabe Y. Serial inbreeding of rabbits with hereditary hyperlipidemia (WHHL-rabbit). Atherosclerosis 36: 261-268, 1980.

727. Watson AD, Leitinger N, Navab M, Faull KF, Horkko S, Witztum JL, Palinski W, Schwenke D, Salomon RG, Sha W, Subbanagounder G, Fogelman AM, and Berliner JA. Structural identification by mass spectrometry of oxidized phospholipids in minimally oxidized low density lipoprotein that induce monocyte/ endothelial interactions and evidence for their presence in vivo. J Biol Chem 272: 13597-13607, 1997.

728. Watson AD, Subbanagounder G, Welsbie DS, Faull KF, Navab M, Jung ME, Fogelman AM, and Berliner JA. Structural identification of a novel pro-inflammatory epoxyisoprostane phospholipid in mildly oxidized low density lipoprotein. J Biol Chem 274: 24787-24798, 1999.

729. Weber C, Schober A, and Zernecke A. Chemokines: key regulators of mononuclear cell recruitment in atherosclerotic vascular disease. Arterioscler Thromb Vasc Biol 24: 1997-2008, 2004.

730. Weiner HL. Induction and mechanism of action of transforming growth factor-beta-secreting Th3 regulatory cells. Immunol Rev 182: 207-214, 2001.

731. Weissberg PL, Clesham GJ, and Bennett MR. Is vascular smooth muscle cell proliferation beneficial? Lancet 347: 305-307, 1996.

732. Welte T, Zhang SS, Wang T, Zhang Z, Hesslein DG, Yin Z, Kano A, Iwamoto Y, Li E, Craft JE, Bothwell AL, Fikrig E, Koni PA, Flavell RA, and Fu XY. STAT3 deletion during hematopoiesis causes Crohn's disease-like pathogenesis and lethality: a critical role of STAT3 in innate immunity. Proc Natl Acad Sci USA 100: 1879-1884, 2003.

733. Wesemann DR, Dong Y, O'Keefe GM, Nguyen VT, and Benveniste EN. Suppressor of cytokine signaling 1 inhibits cytokine induction of CD40 expression in macrophages. J Immunol 169: 2354-2360, 2002.

734. Weston CR and Davis RJ. The JNK signal transduction pathway. Curr Opin Genet Dev 12: 14-21, 2002.

735. Whitman SC, Ravisankar P, and Daugherty A. Interleukin-18 enhances atherosclerosis in apolipoprotein $\mathrm{E}-/-$ mice through release of interferon- $\gamma$. Circ Res 90: e17-e22, 2002.

736. Whitman SC, Ravisankar P, Elam H, and Daugherty A. Exogenous interferon-gamma enhances atherosclerosis in apolipoprotein E-/- mice. Am J Pathol 157: 1819-1824, 2000.

737. Wick G, Kleindienst R, Schett G, Amberger A, and Xu QB. Role of heat shock protein 65/60 in the pathogenesis of atherosclerosis. Int Arch Allergy Immunol 107: 130-131, 1995.

738. Wick G, Knoflach M, and Xu Q. Autoimmune and inflammatory mechanisms in atherosclerosis. Annu Rev Immunol 22: 361-403, 2004.

739. Wilcox JN, Nelken NA, Coughlin SR, Gordon D, and Schall TJ. Local expression of inflammatory cytokines in human atherosclerotic plaques. J Atheroscler Thromb 1 Suppl 1: S10-S13, 1994.

740. Wilcox JN, Smith KM, Williams LT, Schwartz SM, and Gordon D. Platelet-derived growth factor mRNA detection in human atherosclerotic plaques by in situ hybridization. $J$ Clin Invest 82: 1134-1143, 1988

741. Wildin RS, Ramsdell F, Peake J, Faravelli F, Casanova JL, Buist N, Levy-Lahad E, Mazzella M, Goulet O, Perroni L, Bricarelli FD, Byrne G, McEuen M, Proll S, Appleby M, and Brunkow ME. X-linked neonatal diabetes mellitus, enteropathy and endocrinopathy syndrome is the human equivalent of mouse scurfy. Nat Genet 27: 18-20, 2001.

742. Willis D, Moore AR, Frederick R, and Willoughby DA. Heme oxygenase: a novel target for the modulation of the inflammatory response. Nat Med 2: 87-90, 1996.

743. Witztum JL and Steinberg D. The oxidative modification hypothesis of atherosclerosis: does it hold for humans? Trends Cardiovasc Med 11: 93-102, 2001. 
744. Wojciak-Stothard B, Entwistle A, Garg R, and Ridley AJ. Regulation of TNF-alpha-induced reorganization of the actin cytoskeleton and cell-cell junctions by Rho, Rac, and Cdc42 in human endothelial cells. J Cell Physiol 176: 150-165, 1998.

745. Wolf G, Ziyadeh FN, Thaiss F, Tomaszewski J, Canon RJ, Wenzel V, Zahner G, Helmchen U, and Stahl RA. Angiotensin II stimulates expression of the chemokine RANTES in rat glomerular endothelial cells. Role of angiotensin type 2 receptor. J Clin Invest 100: 1047-1058, 1997.

746. Wong BW, Wong D, and McManus BM. Characterization of fractalkine (CX3CL1) and CX3CR1 in human coronary arteries with native atherosclerosis, diabetes mellitus, and transplant vascular disease. Cardiovasc Pathol 11: 332-338, 2002.

747. Woods A, Brull DJ, Humphries SE, and Montgomery HE. Genetics of inflammation and risk of coronary artery disease: the central role of interleukin-6. Eur Heart J 21: 1574-1583, 2000.

748. Wormald S and Hilton DJ. Inhibitors of cytokine signal transduction. J Biol Chem 279: 821-824, 2004.

749. Wright BH, Corton JM, El-Nahas AM, Wood RF, and Pockley AG. Elevated levels of circulating heat shock protein 70 (Hsp 70) in peripheral and renal vascular disease. Heart Vessels 15: 18-22, 2000.

750. Wuttge DM, Eriksson P, Sirsjo A, Hansson GK, and Stemme S. Expression of interleukin-15 in mouse and human atherosclerotic lesions. Am J Pathol 159: 417-423, 2001.

751. Wuttge DM, Zhou X, Sheikine Y, Wagsater D, Stemme V, Hedin U, Stemme S, Hansson GK, and Sirsjo A. CXCL16/SRPSOX is an interferon-gamma-regulated chemokine and scavenger receptor expressed in atherosclerotic lesions. Arterioscler Thromb Vasc Biol 24: 750-755, 2004.

752. Xia D and Samols D. Transgenic mice expressing rabbit C-reactive protein are resistant to endotoxemia. Proc Natl Acad Sci USA 94: 2575-2580, 1997.

753. Xing Z, Gauldie J, Cox G, Baumann H, Jordana M, Lei XF, and Achong MK. IL-6 is an anti-inflammatory cytokine required for controlling local or systemic acute inflammatory responses. J Clin Invest 101: 311-320, 1998.

754. Xu B, Bhattacharjee A, Roy B, Xu HM, Anthony D, Frank DA, Feldman GM, and Cathcart MK. Interleukin-13 induction of 15lipoxygenase gene expression requires p38 mitogen-activated protein kinase-mediated serine 727 phosphorylation of Stat1 and Stat3. Mol Cell Biol 23: 3918-3928, 2003.

755. Xu CP, Glagov S, Zatina MA, and Zarins CK. Hypertension sustains plaque progression despite reduction of hypercholesterolemia. Hypertension 18: 123-129, 1991.

756. Xu H, Barnes GT, Yang Q, Tan G, Yang D, Chou CJ, Sole J, Nichols A, Ross JS, Tartaglia LA, and Chen H. Chronic inflammation in fat plays a crucial role in the development of obesityrelated insulin resistance. J Clin Invest 112: 1821-1830, 2003.

757. Xu Q, Dietrich H, Steiner HJ, Gown AM, Schoel B, Mikuz G, Kaufmann SH, and Wick G. Induction of arteriosclerosis in normocholesterolemic rabbits by immunization with heat shock protein 65. Arterioscler Thromb 12: 789-799, 1992.

758. Xu Q, Kleindienst R, Waitz W, Dietrich H, and Wick G. Increased expression of heat shock protein 65 coincides with a population of infiltrating $\mathrm{T}$ lymphocytes in atherosclerotic lesions of rabbits specifically responding to heat shock protein 65 . J Clin Invest 91: 2693-2702, 1993.

759. Xu Q, Schett G, Perschinka H, Mayr M, Egger G, Oberhollenzer F, Willeit J, Kiechl S, and Wick G. Serum soluble heat shock protein 60 is elevated in subjects with atherosclerosis in a general population. Circulation 102: 14-20, 2000.

760. Xu XH, Shah PK, Faure E, Equils O, Thomas L, Fishbein MC, Luthringer D, Xu XP, Rajavashisth TB, Yano J, Kaul S, and Arditi M. Toll-like receptor-4 is expressed by macrophages in murine and human lipid-rich atherosclerotic plaques and upregulated by oxidized LDL. Circulation 104: 3103-3108, 2001

761. Yachie A, Niida Y, Wada T, Igarashi N, Kaneda H, Toma T, Ohta K, Kasahara Y, and Koizumi S. Oxidative stress causes enhanced endothelial cell injury in human heme oxygenase-1 deficiency. J Clin Invest 103: 129-135, 1999.

762. Yamaoka S, Courtois G, Bessia C, Whiteside ST, Weil R, Agou F, Kirk HE, Kay RJ, and Israel A. Complementation cloning of
NEMO, a component of the $\mathrm{I} \kappa \mathrm{B}$ kinase complex essential for NF- $\kappa \mathrm{B}$ activation. Cell 93: 1231-1240, 1998.

763. Yamashita H, Shimada K, Seki E, Mokuno H, and Daida H. Concentrations of interleukins, interferon, and C-reactive protein in stable and unstable angina pectoris. Am J Cardiol 91: 133-136, 2003.

764. Yamauchi T, Kamon J, Waki H, Imai Y, Shimozawa N, Hioki K, Uchida S, Ito Y, Takakuwa K, Matsui J, Takata M, Eto K, Terauchi Y, Komeda K, Tsunoda M, Murakami K, Ohnishi Y, Naitoh T, Yamamura K, Ueyama Y, Froguel P, Kimura S, Nagai R, and Kadowaki T. Globular adiponectin protected $o b / o b$ mice from diabetes and ApoE-deficient mice from atherosclerosis. $\mathrm{J} \mathrm{Biol}$ Chem 278: 2461-2468, 2003.

765. Yamawaki H, Lehoux S, and Berk BC. Chronic physiological shear stress inhibits tumor necrosis factor-induced proinflammatory responses in rabbit aorta perfused ex vivo. Circulation 108: 1619-1625, 2003

766. Yamawaki H, Pan S, Lee RT, and Berk BC. Fluid shear stress inhibits vascular inflammation by decreasing thioredoxin-interacting protein in endothelial cells. J Clin Invest 115: 733-738, 2005.

767. Yan SF, Ramasamy R, Naka Y, and Schmidt AM. Glycation, inflammation, and RAGE: a scaffold for the macrovascular complications of diabetes and beyond. Circ Res 93: 1159-1169, 2003.

768. Yang DD, Kuan CY, Whitmarsh AJ, Rincon M, Zheng TS, Davis RJ, Rakic P, and Flavell RA. Absence of excitotoxicity-induced apoptosis in the hippocampus of mice lacking the Jnk3 gene. Nature 389: 865-870, 1997

769. Yang X, Coriolan D, Murthy V, Schultz K, Golenbock DT, and Beasley D. Proinflammatory phenotype of vascular smooth muscle cells: role of efficient toll-like receptor 4 signaling. Am J Physiol Heart Circ Physiol 289: H1069-H1076, 2005.

770. Yang XL, Khosravifar R, Chang HY, and Baltimore D. Daxx, a novel Fas-binding protein that activates JNK and apoptosis. Cell 89: 1067-1076, 1997.

771. Yang Z, Chen M, Fialkow LB, Ellett JD, Wu R, and Nadler JL. Inhibition of STAT4 activation by lisofylline is associated with the protection of autoimmune diabetes. Ann NY Acad Sci 1005: 409411, 2003

772. Yao LB, Pan JL, Setiadi H, Patel KD, and McEver RP. Interleukin 4 or oncostatin $\mathrm{M}$ induces a prolonged increase in P-selectin mRNA and protein in human endothelial cells. J Exp Med 184: 81-92, 1996.

773. Yeh M, Gharavi NM, Choi J, Hsieh X, Reed E, Mouillesseaux KP, Cole AL, Reddy ST, and Berliner JA. Oxidized phospholipids increase interleukin 8 (IL-8) synthesis by activation of the c-src/signal transducers and activators of transcription (STAT) 3 pathway. J Biol Chem 279: 30175-30181, 2004.

774. Yla-Herttuala S, Lipton BA, Rosenfeld ME, Sarkioja T, Yoshimura T, Leonard EJ, Witztum JL, and Steinberg D. Expression of monocyte chemoattractant protein-1 in macrophage-rich areas of human and rabbit atherosclerotic lesions. Proc Natl Acad Sci USA 88: 5252-5256, 1991.

775. Yla-Herttuala S, Palinski W, Butler SW, Picard S, Steinberg D, and Witztum JL. Rabbit and human atherosclerotic lesions contain IgG that recognizes epitopes of oxidized LDL. Arterioscler Thromb 14: 32-40, 1994.

776. Yokota T, Shimokado K, Kosaka C, Sasaguri T, Masuda J, and Ogata J. Mitogenic activity of interferon gamma on growth-arrested human vascular smooth muscle cells. Arterioscler Thromb 12: 1393-1401, 1992.

777. Yonemura A, Momiyama Y, Fayad ZA, Ayaori M, Ohmori R, Higashi K, Kihara T, Sawada S, Iwamoto N, Ogura M, Taniguchi $H$, Kusuhara $M$, Nagata $M$, Nakamura H, Tamai S, and Ohsuzu F. Effect of lipid-lowering therapy with atorvastatin on atherosclerotic aortic plaques detected by noninvasive magnetic resonance imaging. J Am Coll Cardiol 45: 733-742, 2005.

778. Youssef S, Stuve O, Patarroyo JC, Ruiz PJ, Radosevich JL, Hur EM, Bravo M, Mitchell DJ, Sobel RA, Steinman L, and Zamvil SS. The HMG-CoA reductase inhibitor, atorvastatin, promotes a Th2 bias and reverses paralysis in central nervous system autoimmune disease. Nature 420: 78-84, 2002. 
779. Yu $\mathbf{Q}$ and Stamenkovic I. Cell surface-localized matrix metalloproteinase-9 proteolytically activates TGF-beta and promotes tumor invasion and angiogenesis. Genes Dev 14: 163-176, 2000.

780. Yun JJ, Fischbein MP, Laks H, Irie Y, Espejo ML, Fishbein MC, Berliner JA, and Ardehali A. Rantes production during development of cardiac allograft vasculopathy. Transplantation 71: 1649-1656, 2001.

781. Zahn R, Schneider S, Frilling B, Seidl K, Tebbe U, Weber M, Gottwik M, Altmann E, Seidel F, Rox J, Hoffler U, Neuhaus KL, and Senges J. Antibiotic therapy after acute myocardial infarction: a prospective randomized study. Circulation 107: 1253$1259,2003$.

782. Zandi E, Chen Y, and Karin M. Direct phosphorylation of IkappaB by IKKalpha and IKKbeta: discrimination between free and NF-kappaB-bound substrate. Science 281: 1360-1363, 1998.

783. Zhang F, Basinski MB, Beals JM, Briggs SL, Churgay LM, Clawson DK, DiMarchi RD, Furman TC, Hale JE, Hsiung HM, Schoner BE, Smith DP, Zhang XY, Wery JP, and Schevitz RW. Crystal structure of the obese protein leptin-E100. Nature 387: 206-209, 1997.

784. Zhang SH, Reddick RL, Piedrahita JA, and Maeda N. Spontaneous hypercholesterolemia and arterial lesions in mice lacking apolipoprotein-E. Science 258: 468-471, 1992.

785. Zhang Y and Chen F. Reactive oxygen species (ROS), troublemakers between nuclear factor-kappaB (NF-kappaB) and c-Jun NH(2)-terminal kinase (JNK). Cancer Res 64: 1902-1905, 2004.

786. Zhao L, Cuff CA, Moss E, Wille U, Cyrus T, Klein EA, Pratico D, Rader DJ, Hunter CA, Pure E, and Funk CD. Selective interleukin-12 synthesis defect in 12/15-lipoxygenase-deficient macrophages associated with reduced atherosclerosis in a mouse model of familial hypercholesterolemia. J Biol Chem 277: 3535035356,2002

787. Zhao L, Moos MP, Grabner R, Pedrono F, Fan J, Kaiser B, John N, Schmidt S, Spanbroek R, Lotzer K, Huang L, Cui J, Rader DJ, Evans JF, Habenicht AJ, and Funk CD. The 5-lipoxy- genase pathway promotes pathogenesis of hyperlipidemia-dependent aortic aneurysm. Nat Med 10: 966-973, 2004.

788. Zheng Y, Saftig P, Hartmann D, and Blobel C. Evaluation of the contribution of different ADAMs to tumor necrosis factor alpha (TNFalpha) shedding and of the function of the TNFalpha ectodomain in ensuring selective stimulated shedding by the TNFalpha convertase (TACE/ADAM17). J Biol Chem 279: 42898-42906, 2004.

789. Zhou X, Caligiuri G, Hamsten A, Lefvert AK, and Hansson GK. LDL immunization induces T-cell-dependent antibody formation and protection against atherosclerosis. Arterioscler Thromb Vasc Biol 21: 108-114, 2001.

790. Zhou X, Nicoletti A, Elhage R, and Hansson GK. Transfer of CD4(+) T cells aggravates atherosclerosis in immunodeficient apolipoprotein E knockout mice. Circulation 102: 2919-2922, 2000.

791. Zhou X, Paulsson G, Stemme S, and Hansson GK. Hypercholesterolemia is associated with a T helper (Th) $1 / \mathrm{Th} 2$ switch of the autoimmune response in atherosclerotic apo E-knockout mice. J Clin Invest 101: 1717-1725, 1998.

792. Zhu J, Quyyumi AA, Rott D, Csako G, Wu H, Halcox J, and Epstein SE. Antibodies to human heat-shock protein 60 are associated with the presence and severity of coronary artery disease: evidence for an autoimmune component of atherogenesis. Circulation 103: 1071-1075, 2001.

793. Zhu Y, Lin JH, Liao HL, Verna L, and Stemerman MB. Activation of ICAM-1 promoter by lysophosphatidylcholine: possible involvement of protein tyrosine kinases. Biochim Biophys Acta 1345: 93-98, 1997.

794. Zuckerman SH, Evans GF, and O'Neal L. Cytokine regulation of macrophage apo E secretion: opposing effects of GM-CSF and TGF- $\beta$. Atherosclerosis 96: 203-214, 1992.

795. Zwacka RM, Zhou W, Zhang Y, Darby CJ, Dudus L, Halldorson J, Oberley L, and Engerlhardt JF. Redox gene therapy for ischaemia/reperfusion injury of the liver reduces AP-1 and NF- $\kappa \mathrm{B}$ activation. Nature Med 4: 698-704, 1998. 